\title{
From the margins to the mainstream: imagining socioecological futures in Wales
}

\section{Pigott, Anna}

How to cite:

Pigott, Anna (2018) From the margins to the mainstream: imagining socioecological futures in Wales. Doctoral thesis, Swansea University.

http://cronfa.swan.ac.uk/Record/cronfa40937

Use policy:

This item is brought to you by Swansea University. Any person downloading material is agreeing to abide by the terms of the repository licence: copies of full text items may be used or reproduced in any format or medium, without prior permission for personal research or study, educational or non-commercial purposes only. The copyright for any work remains with the original author unless otherwise specified. The full-text must not be sold in any format or medium without the formal permission of the copyright holder. Permission for multiple reproductions should be obtained from the original author.

Authors are personally responsible for adhering to copyright and publisher restrictions when uploading content to the repository.

Please link to the metadata record in the Swansea University repository, Cronfa (link given in the citation reference above.)

http://www.swansea.ac.uk/library/researchsupport/ris-support/ 


\section{From the margins to the mainstream: imagining socioecological futures in Wales}

Anna Pigott

Submitted to Swansea University in fulfilment of the requirements for the degree of Doctor of Philosophy.

Swansea University

2018 



\section{Declaration}

This work has not previously been accepted in substance for any degree and is not being concurrently submitted in candidature for any degree.

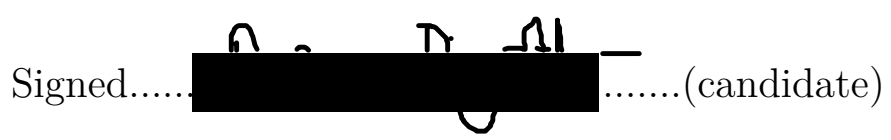

Date...........................

\section{Statement 1}

This thesis is the result of my own investigations, except where otherwise stated. Where correction services have been used, the extent and nature of the correction is clearly marked in a footnote(s).

Other sources are acknowledged by footnotes giving explicit references. A bibliography is appended.

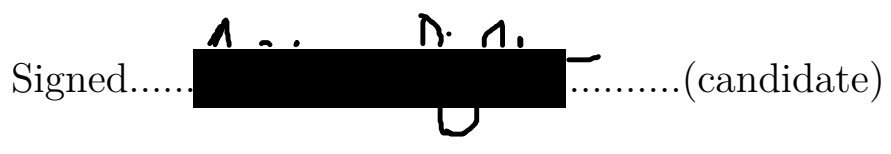

Date........14tḥ. Jụne 2018

\section{Statement 2}

I hereby give consent for my thesis, if accepted, to be available for photocopying and for inter-library loan, and for the title and summary to be made available to outside organisations.

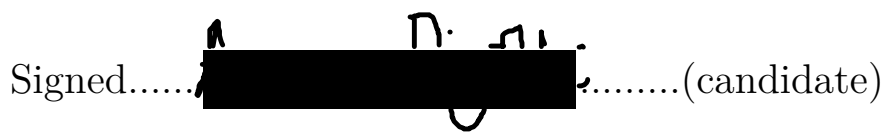

Date..........14th June 2018 


\section{Abstract}

In light of widespread claims about a lack of imagination in response to socioecological crises, this study explores various projects in Wales in order to critically examine the kinds of imaginaries of socioecological transformation that these projects are generating. The Welsh Government's pioneering Well-being of Future Generations Act (2015) provides the context for the research, and this study presents the first critical analysis of this new legislation, and the imaginaries associated with it. Alongside this analysis, however, the study also takes more marginal projects and practices seriously as forms of "minor theory" that might present alternative ways of doing things. To this end, the study has engaged with a range of projects that are envisioning socioecological transformation, including projects in the arts, alternative agriculture, and renewable energy.

The study thus engages with imaginaries of socioecological transformation "from the margins to the mainstream", not by positing these imaginaries as "unimportant" as opposed to "important" (respectively), but by seeking to give equal attention to the political potential of the kinds of ideas that are in play, and what kinds of socioecological futures these ideas make possible. Collective imaginaries of socioecological crises, and the ideas that sustain them, are an important field of struggle with regard to how particular forms of transformation are, or are not, set in motion. The thesis explores three main themes: time and futurity, human environment relations, and the role of art in socioecological transformation, and shows how notions of complexity, non-linearity, and more-than-human agency emerge as important ideas, and often in unexpected or overlooked places. The research is based on combinations of participant observation, interview, and document analysis, and adopts a standpoint that research does not simply represent how worlds are made, but also participates in their making. 


\section{Contents}

Abstract .............................

List of figures ..................... . . . ix

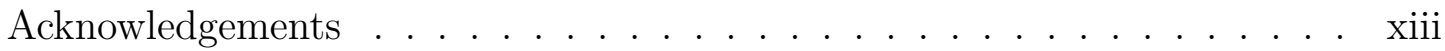

Dedication . . . . . . . . . . . . . . . . $\mathrm{xv}$

1 Introduction $\quad 1$

1.1 Genesis of the $\mathrm{PhD} \ldots \ldots \ldots \ldots 1$

1.2 Research rationale . . . . . . . . . . . . . . . 3

1.2.1 Socioecological transformation . . . . . . . . . . . . 5

1.2.2 "Changing the intellectual climate" . . . . . . . . . . . . . 10

1.2 .3 Why Wales? . . . . . . . . . . . . . . . . . . . 12

1.3 Research context . . . . . . . . . . . . . . . . 16

1.3 .1 Imagination . . . . . . . . . . . . . . . . . 17

1.3.2 Utopia, dystopia, and the future . . . . . . . . . . . 20

1.3 .3 Stories . . . . . . . . . . . . . . . . . . . . . . . . 27

1.3.4 The Anthropocene . . . . . . . . . . . . . . . . . 32

1.4 Research questions . . . . . . . . . . . . . . . . 35

1.5 Thesis structure . . . . . . . . . . . . . . . . . 37

2 Ways of knowing, ways of working $\quad 39$

2.1 Ways of knowing . . . . . . . . . . . . . . . . . . 39

2.1.1 Utopia as (my) method . . . . . . . . . . . . . . . . . 41

2.1.2 Analysis through stories, narratives, and "Earth-writing" . . . 44

2.1.3 Truth . . . . . . . . . . . . . . . . 47

2.2 Who with, where, and why . . . . . . . . . . . . . 49

2.2.1 Perspectives on place . . . . . . . . . . . . . . . . . . . . 49

2.2.2 Case study choice . . . . . . . . . . . . . . . . . . . . . . . . . . . . . . . . . 53

2.2 .3 Welsh Government . . . . . . . . . . . . . . . 54

2.2.4 Ghost Forest environmental art installation . . . . . . . . . 56

2.2.5 Swansea City Council . . . . . . . . . . . . . . . . . 57

2.2 .6 Zero Carbon Britain . . . . . . . . . . . . . . . . . 58

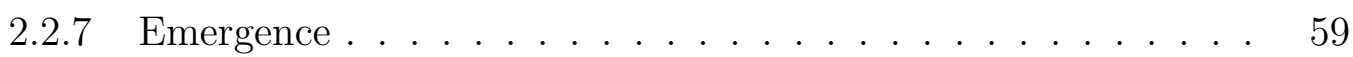

2.2 .8 Cae Tan . . . . . . . . . . . . . . . . 60

2.3 Ways of working . . . . . . . . . . . . . . . 61

2.3.1 Conversation as research method (a.k.a In-depth interviews) . 63

2.3.2 Vox-pops..................... 68 
2.3.3 Participant observation . . . . . . . . . . . . . . . . . 69

2.3.4 Document analysis . . . . . . . . . . . . . . . . . 71

2.3.5 Analysis . . . . . . . . . . . . . . . . 73

2.4 Introducing the empirical chapters . . . . . . . . . . . . . . 75

3 Constructing time to build the future $\quad 77$

3.1 Transformative visions . . . . . . . . . . . . . . . . . . 79

3.1 .1 Welsh Government . . . . . . . . . . . . . . . . 79

3.1.2 Zero Carbon Britain . . . . . . . . . . . . . . . . . . . 81

3.1 .3 Emergence .................... 83

3.2 Imagine that: attitudes towards change and uncertainty . . . . . . . . 84

3.2.1 Resilience ... . . . . . . . . . . . . . 85

3.2.1.1 Vignette: Swansea Council and the future . . . . . 89

3.2.2 Embracing uncertainty . . . . . . . . . . . . . . . 94

3.3 Temporal trajectories . . . . . . . . . . . . . . . . . . . . . . 99

3.3.1 Linear time . . . . . . . . . . . . . . . . . . . 99

3.3.2 Emergent time . . . . . . . . . . . . . . . . 104

3.4 The role of knowledge . . . . . . . . . . . . . . . . . . . . 108

3.4.1 A brief consideration of consensus . . . . . . . . . . . . 112

3.5 The question of looking ahead . . . . . . . . . . . . . . . . . 114

3.6 Concluding thoughts and some tensions explored . . . . . . . . . . . 124

4 Cultures of nature: imagining and performing more-than-human worlds

4.1 Introduction . . . . . . . . . . . . . . . . . . . . . . 131

4.1.1 Imagining "nature" . . . . . . . . . . . . . . . . . . . . 133

4.1.2 Performing socio-ecologies . . . . . . . . . . . . . . 137

4.2 Cae $\operatorname{Tan} \ldots \ldots \ldots \ldots$

4.2 .1 Matter and energy . . . . . . . . . . . . . . . . . . 140

4.2.2 Ritual and spirituality . . . . . . . . . . . . . . . . . 146

4.2 .3 Hocus Pocus . . . . . . . . . . . . . . . . . . . . 157

4.3 Welsh Government . . . . . . . . . . . . . . . . . . . 164

4.3.1 Environmental limits . . . . . . . . . . . . . . . 166

4.3 .2 Ecosystem services . . . . . . . . . . . . . . . . . 168

4.3 .3 Making nature work . . . . . . . . . . . . . . . . . . . . . 174

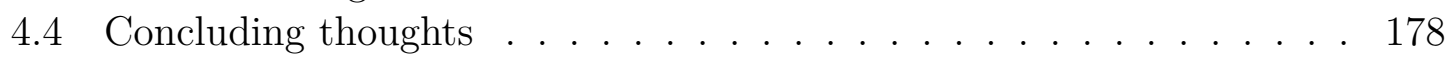

4.4.1 Vexing vocabulary . . . . . . . . . . . . . . . 179

4.4 .2 Thinking differently . . . . . . . . . . . . . . . . . . . . 181

5 The role of art in socioecological transformation $\quad \mathbf{1 8 5}$

5.1 Introduction . . . . . . . . . . . . . . . . . . . . 185

5.1.1 A place for art? . . . . . . . . . . . . . . . . 186

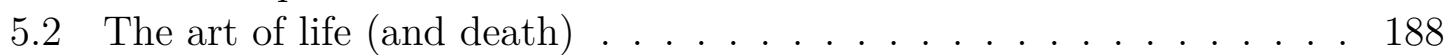

5.2.1 A ghostly presence . . . . . . . . . . . . . . . . 188

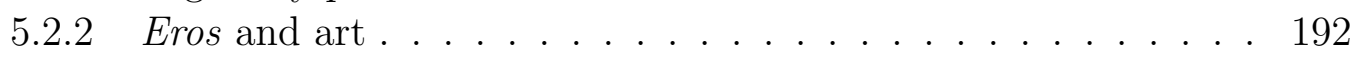

5.3 A sense of place . . . . . . . . . . . . . . 197 
5.3.1 The Walk That Reconnects . . . . . . . . . . . . . . . . . 200

5.3.2 "Topologies" of environmental art . . . . . . . . . . . . . 202

5.4 The art of agency . . . . . . . . . . . . . . . . . . 208

5.5 Re-imagining environmental art? . . . . . . . . . . . . 212

5.5.1 Re-thinking "the artist" . . . . . . . . . . . . . . 213

5.5.2 Re-defining "art" . . . . . . . . . . . . . . . . 215

5.5.3 Geopoetics and indigenous ecopoetics . . . . . . . . . . 220

5.5.4 Incommensurability . . . . . . . . . . . . . . . 226

5.6 Concluding thoughts on art and socioecological transformation . . . . 229

6 Reflections $\quad 233$

6.1 Key contributions . . . . . . . . . . . . . . . 235

6.1 Time and futurity . . . . . . . . . . . . . . . 235

6.1.2 Realising relationality . . . . . . . . . . . . . . 237

6.1.3 The role of art in socioecological transformation . . . . . . . . 238

6.2 Overarching themes . . . . . . . . . . . . . . . . . . . 240

6.2.1 "Social physics" . . . . . . . . . . . . . . . . . . 240

6.2.2 The good, the bad, or the post-Anthropocene? . . . . . . . . . 243

6.3 Limitations . . . . . . . . . . . . . . . . . . . . 245

6.4 Concluding thoughts . . . . . . . . . . . . . . . . 247

$\begin{array}{ll}\text { Bibliography } & 251\end{array}$ 


\section{List of Figures}

3.1 Welsh Government initiatives . . . . . . . . . . . . . . . . . 82

3.2 Philip Ralph with participants at COP123 . . . . . . . . . . . . 96

3.3 ZCB scenario trajectory . . . . . . . . . . . . . . . . . . . 100

3.4 Diagrams of temporality . . . . . . . . . . . . . . . 107

3.5 Positive Future . . . . . . . . . . . . . . . . . . . . 116

3.6 Fossil-fuelled dream . . . . . . . . . . . . . . . . . . . . . . . . . . . . . 119

3.7 ZCB film stills . . . . . . . . . . . . . . . . . . . . . . . . . . . 119

3.8 Welsh Government film stills . . . . . . . . . . . . . . . . . 120

4.1 Snapshots of life at Cae Tan . . . . . . . . . . . . . . . . . . . 140

4.2 Biodynamic preparation . . . . . . . . . . . . . . . . . . . . 148

4.3 The Sun . . . . . . . . . . . . . . . . . . . . . 155

4.4 Natural resource management film still . . . . . . . . . . . . . . . . 172

5.1 Ghost Forest sites . . . . . . . . . . . . . . . . . . . . . . . 189

5.2 Spirals . . . . . . . . . . . . . . . . . . . . . . . . 194

5.3 Two views of the environment . . . . . . . . . . . . . . 203

5.4 Walking in silence . . . . . . . . . . . . . . . . . . . 210

5.5 Reading a Mohawk gratitude prayer . . . . . . . . . . . . 225 


\section{Acknowledgements}

First of all, a huge thank you to my supervisors, Dave Clarke and Amanda Rogers, without whom this $\mathrm{PhD}$ project would never have existed. Thank you for giving me the opportunity of a lifetime, and for letting me make it my own. You were always there when I needed you, with kind encouragement, wisdom, incredible proof-reading skills (despite being incredibly busy), and an uncanny ability to know exactly where to shine a guiding light and when. Thanks also to the Economic and Social Research Council and Doctoral Training Centre Wales, who funded the PhD and also funded an Overseas Institutional Visit to Arizona State University in 2016. I am enormously grateful to Manjana Milkoreit for hosting me during this visit, and to all those who spared me their time, shared their thoughts, and showed me around. This thesis is as much shaped by the Arizonan sun as by the Welsh rain (and, as I write, snow!).

I could never have imagined how my life would have changed when I arrived in Swansea as a nervous MSc student six years ago. Since then, the Wallace building has become something of a home for me, thanks to all the friendly and supportive people in it. To my office mates of $232 \mathrm{a}$ and $232 \mathrm{~b}$, past and present, you're all amazing I will miss our tea walks! Beyond the department, this "ugly, lovely" city feels like home, in large part due to the friends I have made here. I love being part of this international motley crew. I won't name you all for fear of missing someone out, but you know who you are. Thank you for having me.

My heartfelt thanks goes to all those who have participated in this research. You've taught me so much more than theory. Special thanks to Fern Smith and Philip Ralph my involvement with Emergence has been a true eye-opener, and I will be eternally grateful for how you welcomed me and shared your ideas so openly. Fern, our conversations over the past four years have been and I'm sure will continue to be! foundational to so much. Thank you. And speaking of life lessons "cheers" to Edie, our accidentally-on-purpose feline housemate. I once read somewhere that "there are few Zen masters in the world: all of them cats". Trying to emulate your supreme ability to "just be" is perhaps the best lesson of them all.

Thank you to Ubuntu an inspirational example of technology-for-good! This thesis would have been a very different (much more stressful) beast had it not been for the aid of this free, open-source, communally-built operating system.

And finally thank you to my family. To my brothers, Max and Ivan: your humour, creativity, and compassion continually inspires me, even at long distances, thank you for being you! To Mum and Dad, well, "thanks" doesn't really cut it, does it? There really are no words to express my gratitude for your unflagging support, encouragement, and love. You are simply the best. And to Tadas Nikonovas, my best friend, inspiration, everything-support, and partner through it all: ačiū už viską, aš tave myliu, einame i lauką.

Anna Pigott, Swansea, 10th December 2017 
For my parents, Gillian and Nigel 


\section{Chapter 1}

\section{Introduction}

$[\mathrm{H}]$ istory is shaped by the groundswells and common dreams that single acts and moments only represent. It's a landscape more complicated than commensurate cause and effect. Politics is a surface in which transformation comes about as much because of pervasive changes in the depths of the collective imagination as because of visible acts, though both are necessary. And though huge causes sometimes have little effect, tiny ones occasionally have huge consequences. (Solnit 2003, 1)

\subsection{Genesis of the $\mathrm{PhD}$}

"Beginnings are always arbitrary, always imagined", say Anderson and Harrison $(2010,3)$. In one sense, this $\mathrm{PhD}$ began in 2013 when I took up the studentship and made my first tentative steps into the world of postgraduate research. In another sense, this thesis is the culmination (although I'm sure "culminations" are always arbitrary, always imagined, too!) of a quest for answers to questions which began much earlier in my life. At first, these might not have manifested themselves as fully-formed questions. More likely, they started as feelings of unease, for example, while watching wildlife documentaries on television, as I frequently did during my childhood. Unease because, while the wonder and the thrill of the images captivated me, I was also struck by feelings of sadness and despair. After all, when David Attenborough tells you the animals are in trouble, you know it's bad. At first, and I suspect like many kids enthralled by wildlife documentaries and glossy World Wildlife 
Fund for Nature campaigns in the 1990s, I wanted to be a vet (to save the animals). As it dawned on me that it wasn't only the pandas who were in trouble, and I became more acutely aware of environmental, and humanitarian, crises "writ large", my veterinary ambitions morphed into aspirations of the Earth Warrior variety I wanted, of course, to help "save the planet". But how? (For it would surely have to involve more than rescuing "Snippy" the mouse from the bottom of our flooded dustbin, although that was a particularly proud moment).

I have no doubt that these somewhat embarrassing childhood ambitions steered me to where I now find myself. It has been something of a gradual and convoluted path from those days, and my lofty hopes to save the planet have, I'm sad to say, come quite a long way down to earth by now. But smaller questions have continued to animate my thoughts in relation to the spectre of environmental catastrophe which increasingly seems to saturate life (Chakrabarty 2009). Questions such as "why?" and "what can be done?" and "how could things be otherwise?" For several years as a young adult I dodged these questions by throwing myself into jobs where I could simply be in "nature", rather than have to think much about it. When I did return to studying, I mainly identified myself as a physical geographer, because physical geographers liked the environment and went outdoors, while human geographers preferred humans and stayed in coffee shops or so I thought. My adventures in geography (an undergraduate degree and then an MSc in Climate Change and Environmental Dynamics) and occasional work in sustainability-related fields, helped me to understand the scale and complexity of environmental problems knowledge which served to increase my unease, but left questions about how to change things nagging.

When I embarked on this $\mathrm{PhD}$, I was, therefore, somewhat surprised to find a home for those questions in human geography specifically, in cultural and critical geography. Almost overnight a whole field opened up to me in which I discovered many other voices also asking essentially the same questions! Chiefly, "why are things like they are?" and "how could things be otherwise?" (e.g. Castree et al. 2010). This discovery was elating, and was also pivotal in instigating a wholesale shift in my ways of thinking about "the environment". As Chakrabarty (2009, 201) says, "anthropogenic explanations of climate change spell the collapse of the age-old humanist distinction between natural history and human history" which is easy to say but much more difficult to think and to practise. Cultural and critical geography (particularly, strands in political ecology, and decolonial and feminist geography), provided me with some conceptual tools with which to begin doing so. In particular, I became interested in the possibility that what people think is as important as 
what they do when it comes to the environment, not so much in terms of individual values and attitudes which translate into behaviour change only in very complex and contingent ways (if at all) and which have been studied extensively (e.g. Blake 1999, Kollmuss and Agyeman 2002, Moser and Dilling 2011) but in terms of much broader "geographical imaginations" (Gregory 1994) about social and environmental change.

I became interested in how representations, ideas, thoughts, feelings, affects, and emotions come together to form (admittedly nebulous) social configurations and experiences which shape how lives are lived and how possibilities for socioecological transformations emerge. Williams (1977, 133 34) refers to such configurations as "structures of feeling", which he describes as "social experiences in solution, as distinct from other social semantic formations which have been precipitated and are more evidently and more immediately available." I was excited by the notion that, as indicated in the epigraph above, transformations are infinitely more complex than simple chains of cause and effect. And while this can be daunting, it also presents the tantalising possibility that opportunities for change, however large or small, abound, whether we know it yet or not. As Huehls $(2010,420)$ remarks, "the experiment is not over; relations are murky; we do not know the results". The proposal for this PhD took shape around this idea, with an intention to explore some outcrops of socioecological transformation which are detectable in and around where I live, to think, write, and talk about them, and so to participate in the experiment.

\subsection{Research rationale}

To be honest I've never really thought that far ahead because I think, what you actually do hope ... never really happens.

I don't think about [the future] too much really, I think the extent of our problems are ... so horrific I don't like thinking about it much.

I can't actually deal with it sometimes, I-I shut off, I know, I mostly shut off, 'cos the idea of an orangutan having nowhere to live any more is just too I can't deal with that, it's just too depressing really.

To be honest I don't think people think of the future? I think people think of the here and now, and their day to day problems and issues of 
today.

(Various research participants, Wales, 2014 16)

Initially, this research developed as a response to the widespread assertion that the ways in which people and societies imagine the future, and imagine possibilities for change, are central to actions in the present (e.g. Adam and Groves 2007, Yusoff and Gabrys 2011). My intention was to analyse (positive) visions of the future being produced by environmentally-focussed organisations and the people associated with them, in order to be able to say something of the "groundswells and common dreams" (Solnit 2005, 85) that might influence socioecological transformation. However, it soon became clear that people's capacities to visualise environmental futures, particularly desirable ones, were lacking, as the comments from some of my research participants, above, suggest. In addition, few organisations that I approached had anything as explicit as a concrete "vision of the future" emblazoned on their website (although some did). As a result, I broadened my search to be open to a much more nebulous collection of sayings, doings, affects, agendas and experiences which, even if they weren't explicitly to do with the question of "the future", were nevertheless implicitly imagining and reimagining it by working to change the status quo. My task then became to try to distil something of the kinds of socioecological futures being implied by these imaginaries, to explore any commonalities and differences, and in the spirit of Solnit's epigraph, above pay equal attention to the tiny examples as well as the huge.

In what follows I describe the rationale for the $\mathrm{PhD}$ in more detail. First, I explain how I have approached the notion of "socioecological transformation" (an admittedly very broad term), and how this particular way of thinking about it informs the rationale for this research. Second, I discuss how the research also arose in response to recent calls for human geographers (and other social scientists) to play a more active role in wider debates in environmental change research. Third, I explain the rationale for basing the research in Wales, given the Welsh Government's recent policy innovations on environment and well-being. The second half of the chapter then introduces some of the key contextualising concepts and fields of literature for the research, and finishes with a summary of the research questions and thesis structure. 


\subsubsection{Socioecological transformation}

The term "socioecological" (sometimes "socio-ecological" in the literature) has its origins in the concept of Social-Ecological Systems (SES) in the biological sciences (see Ostrom 2009), although attempts to bring humans and environment together into a more complex, systems view of the world were already apparent (e.g. Von Bertalanffy 1956, Bateson 1979, Berkes and Folke 1998). SES now also characterises approaches to human environment relations in Earth Systems science, and is often used in conjunction with concepts of resilience, complexity, and non-linearity, and as a way of "modelling" earth systems that acknowledges interaction between human and nonhuman realms (see, for example, Costanza et al. 2007, Berkes et al. 2008, Glaser et al. 2008). Thus, Fischer-Kowalski and Haberl (2007, 13) describe socioecological systems as "the overlap of natural and cultural spheres of causation." However, in social sciences and in human geography in particular, the use of "socioecological", which has proliferated of late (see, for example, Braun 2015a, Ginn 2015, Hawkins et al. 2015b, Head 2015, Wainwright and Mann 2015), takes on slightly different meanings. For a start, the word "system" is usually dropped, and "socioecological" seems to be used less to refer to the interaction of otherwise distinguishable realms ("social" and "ecological"), and instead to signpost a way of thinking about these things as thoroughly amalgamated. It is in this latter sense of the word that I use it, because it offers a way to at least try to reflect the collapse, as already mentioned, of a distinction between human and natural histories in Western thought (Chakrabarty 2009), and it avoids the pitfalls of referring to "social" and "environmental" change separately, as though they occurred independently of one another. However, the ambiguity of this usage of "socioecological" warrants further exploration and explanation here.

In human geography "socioecological" tends to be used as something of a "catch-all" phrase which signifies an acknowledgement of (a) the social construction of nature, a proposal which identifies the separate categories of "culture" and "nature" as, essentially, false ones (e.g. Hajer 1995, Escobar 1996, Descola 2013, Castree 2013a), and (b) an attentiveness to recent attempts in geography (and beyond) to conceptualise the world, as it were, "after nature" (Jones 2009, Lorimer 2015, Purdy 2015). These include numerous proposals of terms, such as cyborg (Haraway 1991), hybrid (Whatmore 2002), and assemblage (for an overview, see Anderson and McFarlane 2011), which seek to articulate just how intertwined and interdependent biological, technological, geological, microbial (you name it) worlds are (examples range from human microbial gut flora to Google Glass, to climate change). But "socioecologi- 
cal" seems to have stuck as an umbrella term, ${ }^{1}$ and is used particularly in relation to topics which might have traditionally been filed under the category of "environmental issues" that are in urgent need of a response. For example, some contexts in which the term "socioecological" is used include Mansfield et al.'s question "[w]hat do environmental politics look like when the environment at stake is understood to be fundamentally socioecological?" (Mansfield et al. 2015, 285) and Hawkins et al.'s similar question, "[w] hat might it mean to consider socioecological transformations and futures from a perspective that deploys an expanded appreciation of the social, one that incorporates the "lively processes and impure forms coexisting in inhabited landscapes'?" (Hawkins et al. 2015b, 331, quoting Lorimer 2012, 594). Given these various engagements with the term, it seems that "socioecological" is not only used as a descriptor but also as a signpost (in its usage in human geography, at least), to point the way towards new or desirable assemblages of human and more-than-human life, and ways of organising. As Hawkins et al. (2015b, 331) add, the challenge is to bring about recognition of ecology not as "interaction between preordained life forms", but rather in terms of their emergence and transformation.

Indeed, "transformation" is a word which is commonly appended to "socioecological" in the literature (see, for example, Braun 2015a, Hawkins et al. 2015b, Kallis and March 2015, Moore et al. 2015). The popularity of the term "socioecological transformation" might be attributed, in part, to its ability to capture something of a zeitgeist; it is a "big picture" concept which reflects, as Wainwright and Mann (2015, 314) contend, "a moment of transition in the planet's history". Braun also uses "socioecological transformation" in relation to a perceived historical juncture, in which two related assumptions are at play; "(1) that socioecological transformations are coming, although the form and shape of what is to come is not easily predicted; and (2) that socioecological changes are also necessary, if we are to avoid the catastrophic futures that appear to be coming toward us" (Braun 2015a, 239).

Herein lies a crucial difference, I suggest, between the concepts of "socioecological transformation" and what might be thought of as its predecessor, "sustainable development". Both have emerged in recognition of the current unsustainablity of the ways in which many human societies organise themselves, and a normative call to change this if, as Braun says (above), we are to avoid catastrophe. However, as I interpret it, "socioecological transformation" implies a much more radical change

\footnotetext{
${ }^{1}$ Its prominence is perhaps ironic given that the word still relies on a binary conception of "social" and "ecological", albeit joined together, although its popularity might be explained by the relative familiarity (and self-explanatory nature) of the term to people outside the niche branches of geography which happen to be well-versed in "cyborg", "hybrid", and "assemblage" geographies.
} 
than has ever been implied by the discourse of sustainable development. Sustainable development, a term which was first coined by the Brundtland Commission's report, Our Common Future (1987), does, indeed, incorporate ideas about linkages between humans and environment, but a key criticism levelled at the discourse of sustainable development is a contention about what is to be sustained. As Garforth $(2002,25)$ comments, "[s] ustainability means continuation; it signifies the possibility that some situation, system, or quality can be maintained indefinitely". Indeed, for many, sustainable development is a contradiction in terms, the emphasis on "development" a disguise for continued economic growth which is simply incompatible with any notion of a sustainable environment (Robinson 2004, Holden 2010). In addition, the notion of development rests on ideas about the linearity and stability of the natural world (in the Brundtland definition, sustainable development is presented as a way of balancing human "needs" with environmental "limits"), and a concurrent narrative of consistent human progress. These are all assumptions which, as Hajer (1995) suggests, reproduce existing paradigms of Western thought that actually limit capacities to imagine genuine alternatives (see also Hector et al. 2014). In contrast, "socioecological transformation" appears to imply some kind of fundamental rupture with the status quo a metamorphosis into something entirely different (Beck 2016). In parallel with developments in the "new sciences" around ideas about ecosystem non-equilibrium and complexity (Zimmerer 1994), the amalgamation of "social" and "ecological", together with the notion of "transformation" (a word which has a different, less predictable, ring to it than "development"), seems to reflect an emerging understanding of the world as constantly changing and in flux. As Mentz, reflecting on this changing ontological landscape, comments,

the era of sustainability is over. Behind our shared cultural narratives of sustainability sits a fantasy about stasis, an imaginary world in which we can trust that whatever happened yesterday will keep happening tomorrow [...] Such a narrative imagines a happy, stable relation between human beings and the nonhuman environment $[\ldots]$ In this sustainable green world, complicated things fit into simple packages [...] that's the dream toward which sustainability entices us. To be sustainable is to persist in time, unchanged in essence if not details. That's not the human experience of the nonhuman world [...] Changing scale matters, and local variation does not preclude global consistency, but the feeling of the world on our skin is disruptive. Our environment changes constantly, unexpectedly, often painfully. (Mentz 2012, 586) 
Socioecological transformation, then, appears to fit the bill as a concept for responding to environmental crises "after sustainability" (Mentz 2012, 586). It is, however, like sustainable development, still a very broad term; it could, arguably, mean almost anything, and so in what follows I describe how I interpret and use the notion of socioecological transformation, and why this is important for understanding my approach to, and analysis of, the research in this thesis.

In the literature, socioecological transformation is rarely explicitly defined, but is most often used to imply some kind of "good" transformation, of the variety that might produce more habitable and desirable futures. ${ }^{2}$ For example, Hawkins et al. $(2015,1)$ use the term socioecological transformation in conjunction with the role of art in "'active experimentations and anticipatory interventions' in the face of the challenges posed by the environmental and social uncertainties of the Anthropocene." This orientation towards experimentation also corresponds with an orientation "to the possibilities and consequences of a 'new earth' and a 'new humanity' that is still to come", rather than to a commitment to conservation, because, as they say, "the world never holds still" (Hawkins et al. 2015, 1, quoting Braun 2006, 219). Elsewhere, Ginn $(2015,351)$ uses the term socioecological transformation in the context of "responding well to unruly earth forces". For Moore et al. (2015), it has to do with progressive forms of knowledge production in schools about human environment relations, and similarly, Buck $(2015,372)$ proposes that "relational practices enable enchantment, and this is part of socioecological transformation". Elsewhere, Kallis and March $(2015,1)$ describe "a project of radical socioecological transformation calling for decolonizing the social imaginary from capitalism's pursuit of endless growth". What these various uses of the concept of socioecological transformation have in common is an attentiveness to plurality, relationality, and complexity, and to experimentation and the possibility of unexpected outcomes. Here, it is possible to detect the influence of feminist and posthumanist thought (see, for example, Strauss 2015 and Hawkins et al. 2015b), as well as new materialist and affective geographies that emphasise the importance of the non-representational, or the more-than-representational in socioecological transformation (see Lorimer 2005). This includes an attentiveness to agency as, broadly, the "creative presence" (Whatmore 1999, 26) of beings, and the capacity of beings to transform, to possess intention, to engender affective and emotional responses (see Jones and Cloke 2008). Importantly, in this sense agency is understood to be something possessed by the more-than-human world, and not exclusively by

\footnotetext{
${ }^{2}$ By this I mean that I have not come across "socioecological transformation" being used to describe situations of worsening socioecological conditions, which could, equally, be described as transformations.
} 
humans.

These are all positions which I also broadly adopt in my references to "socioecological transformation" in this thesis. However, I also want to be explicit about two key assumptions about socioecological transformation which also underpin my approach. The first is a conviction that socioecological crises are, first and foremost, crises of neoliberal capitalism, and, as such, it is the practices, ideologies, institutions and economies which sustain neoliberal capitalism which need to be transformed if there is to be any improvement in socioecological conditions. This is a commonly-held position of the political (often Marxist) "left" (see Smith 1984, Harvey 1996, Castree 2003, Kallis 2011, Klein 2015, Moore 2015). As Castree (drawing on Harvey) puts it, "socio-ecological formations [have] become the "'arteries' through which an invisible process of ceaseless [economic] value expansion operates" (Castree 2005, 233). Guattari ([1989] 2014), too, argues that ecological crises are the direct result of the expansion of new forms of capitalism which, as Jones (2009, 300) puts it, are "denuding cultural, psychological, and ecological diversity to the extent that we are witnessing 'ecocide' on a global scale." Although my approach and analysis of the case studies and themes in this thesis is not always explicitly about the political ecologies of capitalism, it is important that I make clear that I am broadly approaching the issue of socioecological transformation from this perspective, rather than from a perspective which, for example, would prioritise technological "fixes" (such as geoengineering) over changes to economic systems (and the ideologies that sustain them).

The second assumption underpinning my approach to socioecological transformation stems, in particular, from Guattari's proposal that of central importance in any transformation are transformations at the microlevel of what he calls "mental ecology" (Guattari [1989] 2014, 12), that is, "the molecular domains of sensibility, intelligence and desire" (ibid., 18) which are the motors of subjectivity and thus which determine how humans relate to one another and to the more-than-human world. Guattari was inspired by Bateson's (1972) view that there is an ecological struggle for survival taking place in the domain of ideas, and thus the task for anyone concerned with the future of life on Earth is to promote ecologically "good" ideas over "bad" ones. As Conley $(2008,118)$, drawing on Guattari, says "[z]ombie-like subjects, degraded social relations, global misery, ongoing violence and environmental catastrophes are the sad truth of this kind of [capitalist] economy. Bad ideas have taken hold." This attention to ideas, and to Guattari's related assertion that there should be no distinction between the psyche, the socius and the environment, has been foundational to my approach to the case studies in this research, and how I discuss them. As I 
will explain below in more detail, I have chosen to focus as much on the kinds of ideas and imaginaries that each case study produces and reproduces as I have on any kind of (perhaps more measurable) socioecological "impact" they have directly. As Gerlach $(2017 \mathrm{a}, 15)$ puts it, "given the desperation of the late Holocene, societies need to intensify the experimental tenacity of ideas", and with this there is a need to critically engage with the "affective lives" (ibid., 11) of these ideas in terms of the kinds of socioecological futures they make possible. Such an analysis is, essentially, the task I have set myself in this research.

Of course, my whole approach is also underpinned by an assumption a hope, perhaps that socioecological transformation is possible. Whether or not environmental catastrophe can still be averted (if indeed, we are not already in the midst of one) is a question I can't answer. But I think Miles (2014, 29) sums up my own feelings best when he says "perhaps I am lost in a dream world if I imagine a post-capitalist, environmentally just and sustainably joyful society, yet unless I can imagine it I have no way to begin to contribute to it." Bauman's (1999) reminder of the original meaning of "crisis" is also instructive. Rather than taking "crisis" to imply impending disaster, as it is often used today, the etymology of crisis communicates a moment of engagement, in which the time to make decisions that could secure a turn for the better, is now.

\subsection{2 "Changing the intellectual climate"}

Related to my use of "socioecological" as a term which attempts to bring considerations of the "social" and "ecological" together, and to my focus on the role of ideas and imaginaries (more on which below), this research is motivated by, and situated within, a so-called "cultural turn" in relation to the study of the environment. Since the late 1980s, this has seen renewed interest in culture as a lens through which to analyse and understand a multitude of issues and which, in particular, emphasises the contingent and constructed qualities of phenomena (e.g. Bourdieu 1993, Ogborn et al. 2003). Environmental crises are also increasingly being approached as matters of culture. For example, Yusoff and Gabrys (2011), referring specifically to climate change in this instance, observe that, after the disappointments of the 2009 Copenhagen Conference of Parties on Climate Change (COP15) (which failed to achieve any binding commitments), there has been a tacit acceptance that there is a need to re-conceptualise climate change as embedded in everyday practices. As they say, 
[t]his entails an imaginative recasting of climate change as something that is not 'out there' (located in a global imaginary or in distant places such as the Arctic or Sub-Saharan Africa), but as something that has relevance for all cultures across all scales, and thus is something 'in here', entangled in contemporary practices and future possibilities (Yusoff and Gabrys 2011, $517)$.

This cultural turn contrasts with causal models of climate science that "imagine humans as either drivers of climate change or recipients of its effects, rather than as a heterogeneous and differentiated social body with distinct desires, constraints, and imaginations" (ibid.). While it is still common for climate change and other aspects of environmental change to be written about and told in scientific language (Harris $2017 b$ ), the cultural turn that many geographers (and others) have insisted is vital in being able to understand and respond to environmental crises (see, for example, Murdoch 1997, Franklin 2002, Urry 2011 and Castree 2013a) is gradually being reflected in more mainstream approaches to environmental-change research. For example, the Future Earth initiative (which directs global research programs on anthropogenic environmental change ${ }^{3}$ ), emphasises the centrality of "human dimensions" in its strategic research agenda, while the International Group of Funding Agencies for Global Change Research issued the Belmont Challenge in 2009, which explicitly referred to the social sciences and humanities as "missing ingredients"(Hawkins et al. 2015a, 221). Critical social scientists have been quick to note that this opening presents an opportunity for research in the humanities and social sciences to constructively shape environmental change research in the decades to come (Demeritt 2009, O'Brien 2011, Castree 2015c, 2015b, 2015a), by inspiring serious debate about the future of humanity (e.g. Hawkins et al. 2015a), and by helping to introduce new ways of thinking about environmental challenges. But this opportunity also carries with it an imperative to get it right. As Castree (in Hawkins et al. 2015a, 221) warns, the invitations to social scientists extended by organisations such as Future Earth risk merely being taken up as tasks in "filling knowledge gaps" about "human dimensions" of environmental change, as an activity which happens downstream of, and separate from, the environmental science. To do so would miss an opportunity to challenge the questionable ontological assumption, which underpins much current environmental science, that there is "one world out there awaiting complete analysis [...] so that global change scientists can devise appropriate technical and behavioural measures in the realms of mitigation, adaptation, and precaution" (Hawkins et al. 2015a, 221).

${ }^{3}$ Available at http://futureearth.org/. Last accessed 31 October 2017. 
Instead, Castree (2015b, 244) suggests we need to change "the intellectual climate" in order to understand environmental change as something which is, as Yusoff and Gabrys $(2011,519)$ contend, "in here", entangled with social and political practices and imaginings, and not "out there", separate from them. This backdrop of proliferating interest in environmental crises as a cultural phenomenon sets the scene for this thesis, and highlights my interest not necessarily in filling some "humanshaped" gaps in current understandings of environmental change, but in re-working how environmental problems are approached in the first place (see also Castree 2017).

\subsubsection{Why Wales?}

So far, I have outlined some rather global concerns and rationales for this research. The decision to base the research in Wales, a nation with a land area of just under 30,000 square kilometres and a population of approximately 3 million people, might then appear to be something of an incongruous choice. Aside from the fact that researching socioecological imaginaries on a global scale would have been far beyond the reach of this project, and the research therefore needed to be based somewhere (as will be discussed further in chapter 2), Wales is also an interesting and important site for this research because of the Welsh Government's recent policy innovations in environment, sustainability and well-being. This includes a constitutional commitment to sustainable development as a "central organising principle" of Welsh Government since devolution in 1996, and the introduction of a pioneering piece of legislation called the Well-being of Future Generations (Wales) Act (WFGA), which received royal assent on 29 April 2015 and entered into force on 1 April 2016. The Act places a duty on all public services in Wales to act in accordance with seven "Well-being Goals" and "Ways of Working" specified by the Act, and it is the first in the world to enshrine, in law, a duty on public bodies to respect the interests of future generations (Davies 2017).

Although unique, these policy innovations have not emerged out of a vacuum, and reflect some broader, international developments in socioecological transformation. The United Nations (UN), for example, have promoted debate and issued guidance on the theme of intergenerational solidarity in its Secretary General's report on Intergenerational Solidarity and Future Generations (2013). The Welsh Government's national consultation exercise "The Wales We Want", which informed the development of the Act, also echoes the UN's "The World We Want" platform that seeks, 
as it states, to "build a collective vision that will be used directly by the United Nations and World Leaders to plan a new development agenda launching in 2015, one that is based on the aspirations of all citizens![sic]". " In addition, the Welsh Government's decision to use Well-being Goals as a framework for the Act echoes the UN's own "Sustainable Development Goals". ${ }^{5}$ The intention that the Act should provide an overarching framework for public governance in Wales is also consistent with the "process-outcomes" model recommended by the Organisation for Economic Cooperation and Development (OECD) for embedding sustainable development within government (see Davies 2017, 167). Finally, the Welsh Government is a key member of the Regional Governments for Sustainable Development Network (a support and sharing network for subnational and regional governments that are working on sustainability transformations), which it helped found in 2002, and for which it has acted as vice-president between 2015 2017. In April 2015, this involved the Welsh Government hosting academics, lawyers, human rights specialists, civil society organisations, senior UN officials, youth representatives, and representatives of national institutions for an international conference that responded to the aforementioned UN Secretary General's report on Intergenerational Solidarity and Future Generations (2013). In this sense, the Welsh Government might be thought of as creating a "transformative niche" (Moore et al. 2014, 5) where policy innovations can be tested and developed, but which has wider relevance for approaches to socioecological transformation, not only in terms of the kinds of actions taken, but also in terms of the ideas it promotes. What becomes of the WFGA is, therefore, of intense interest to other nations and governments considering similar approaches. As Nikhil Seth, Director of the Division for Sustainable Development at the United Nations, has commented (on the passage of the WFGA), he hopes that "what Wales is doing today the world will do tomorrow." 6

However, due to the relative newness of the Act, there has so far been very little academic scrutiny of it (or indeed, of the Welsh Government's approach to socioecological transformation more generally). Existing research (the work of one author), focusses on the legal implications of the Act, and its potential to effect the kinds of changes it aspires to. Davies's $(2016 ; 2017)$ work is instructive in this regard. He finds that, despite a language of "must" and "shall" and "duties" $(2017,171)$, the Act itself has relatively few "teeth" when it comes to enforcement. As Davies points out, the

\footnotetext{
${ }^{4}$ Available at https://www.worldwewant2030.org/about/. Last accessed 2 November 2017.

${ }^{5}$ Available at https://sustainabledevelopment.un.org/sdgs. Last accessed 2 November 2017.

${ }^{6}$ Available at http://gov.wales/newsroom/environmentandcountryside/2015/150429future-generations-act/?lang=en. Last accessed 2 November 2017.
} 
Act is very much couched in "exhortatory rather than mandatory terms" (ibid.), and its efficacy will depend mainly upon the Future Generation Commissioner's (FGC) and the Auditor General's abilities to hold public bodies to account (via a process of reporting to the National Assembly once every four years), and the political will and personalities of those people (Davies 2016; 2017). In addition, the budget for the office of the FGC (which oversees the implementation of the Act) is relatively small, at $£ 1.46$ million per annum, a figure that, as Davies $(2017,175)$ says, "is smaller even than the budget for the Children's Commissioner for Wales, despite the fact that the [Future Generations Commissioner] is overseeing the 'central organising principle' of Welsh governance".

Given these constraints, the power of the Act will depend largely on the ability of the office of the FGC and the Welsh Government to persuade public bodies to co-operate. Therefore, what it is asking people to do that is, the vision and aspiration for the nation that it is asking people to join is vital. Interestingly, a common refrain I heard from members of the public who participated in this research was that the WFGA was "just words", and that it wouldn't amount to much. Conversely, given the Act's lack of legal power to enforce, it might be that words are, in fact, its most powerful element, with the potential to shape people's imaginaries about possible and desirable futures. As shall be discussed in the following sections, this discursive power can influence the forms that socioecological transformation takes, and warrants close attention not only because the Welsh Government's rhetoric about the WFGA Act (and related policies such as the Environment (Wales) Act 2016) form a "grand, regional narrative" (Murphy 2013, 131) about socioecological transformation that creates the context for other policies and actions in Wales, but also because as Nikhil Seth's comment (above) implies there is international interest in what the Welsh Government is saying. So far, however, there has been no critical engagement with the content of the Act in terms of the kinds of narratives about socioecological futures that it promotes. As far as I am aware, this thesis is the first attempt at doing so, and, as such, opens up a field for future research. It also contributes to ongoing research into new modes of governance that are emerging in response to environmental crises (e.g. Anderson 2010, Braun 2014, Wainwright and Mann 2013).

It should be mentioned that the research presented in this thesis takes a different approach to narrative than has previously be explored in the Welsh context. This includes a piece of research commissioned by the Welsh Government in 2014, carried out by the Climate Outreach and Information Network (COIN), ${ }^{7}$ which assessed the

\footnotetext{
${ }^{7}$ Now Climate Outreach. See https://climateoutreach.org/. Last accessed 2 November 2017.
} 
efficacy of various climate change narratives through workshops with members of the public, and developed recommendations for the Welsh Government's climate change communication based on the findings (Marshall 2014). While this work approached narrative from the perspective of individuals, or groups of individuals (based on socioeconomic indicators), and attempted to identify "successful" or "unsuccessful" ways of communicating climate change, I have approached narrative from a different angle in this thesis. Specifically, my intention has been to scrutinise the narratives that the Welsh Government itself is using in relation to sustainability, and to speculate (based on wider social and critical research and theory) on some of the implications of such narratives for socioecological transformation. In addition, my concern in this thesis is less with public perceptions of environment and climate change in Wales (see Capstick et al. 2013) (although this has formed an element of my interviews with research participants), than with the ways in which the Welsh Government presents these issues. Finally, the research in this thesis is emphatically not trying to predict or forecast environmental futures in the Welsh context (as a previous report by Netherwood (2008) has done), but is rather about how futures are imagined (which might include the influence of prediction and forecast techniques, but is not restricted to them).

This thesis therefore engages with the Welsh Government's policies on sustainability not necessarily, as Gerlach says, to apportion "success or failure to an experimental way of living and governing", but rather to assess the composition and potential of the ideas themselves (Gerlach 2017a, 2252). Gerlach writes in the context of his own engagement with the Ecuadorian government's "experiment in living well" (ibid. 2242); a policy programme and state-wide ethos known as "sumak kawsay" ("living well"), which adopts various aspects of Ecuadorian indigenous cosmologies in order to "challenge neoliberal modes of governance, and disrupt the ontological bifurcation of nature and society" (Gerlach 2017a, 2241). His engagement with this programme, he says, "is motivated by an urgency to critique a state experiment that hangs in the balance". My research with the Welsh Government can be thought of in similar terms, and indeed there are many similarities between Ecuador and Wales with regard to their political aspirations to carve out new legislation for more sustainable futures. In particular, I follow Gerlach's interest in the ways in which the experimental ideas being promoted by the government cultivate particular atmospheres which may or may not be amenable to socioecological transformation. In this sense, the research on the Welsh Government presented in this thesis fits within a wider tradition of thinking about nations as imagined communities which are assembled through a 
host of affective atmospheres (e.g. Anderson 2006a, Anderson 2009, Closs Stephens 2016). Previous research in Wales in this vein includes, for example, Curtis (1986) and Gruffudd (1995), which focuses on atmospheres of nationalism and national identity, but so far no studies have explored the affective dimensions of the Welsh Government's WFGA (and related policies), and the ways in which these contribute to "imagined communities" of socioecological transformation, both national and global.

I have gone into detail here about the relevance of the Welsh Government's policy aspirations because this is important for understanding the context of the research project, and to justify why Wales is an important place to conduct the research. However, the Welsh Government is not the sole focus of this thesis, and as will become clear I have also engaged with several other Welsh organisations and projects that are non-governmental and much smaller in scale. In these instances, the decision to choose case studies in Wales was, in large part, because it afforded an opportunity to compare and contrast "smaller stories" (Lorimer 2003, 200) of socioecological transformation with the Welsh Government's national vision, but also because Wales is where I live, and this closeness-of-experience afforded me the opportunity to "dig where I stand" (Mclntosh 2012, 34) and to learn from the case studies I have been involved with over extended periods of time. This approach to the research will be discussed in more detail in chapter 2 . I will just mention here that although I have sought out some "small[er] stories" of socioecological transformation in Wales, I deliberately chose not to focus on the strong tradition of eco-communities in Wales (Maxey 2003, Halfacree 2011). This is, first, because there is extensive existing research on such communities (in Wales and the rest of the UK) and their histories and visions for socioecological transformation (e.g. Maxey 2003, Halfacree 2006, 2007, 2011, Pickerill and Maxey 2007, Pickerill and Maxey 2009, Andreas and Wagner 2012, Pickerill 2015, Forde 2017) and, second, because I was keen to make space in the research for projects which might be surprising or unexpected, and which did not necessarily fit a stereotypical, predominantly rural, image of "alternative" socioecological transformation.

\subsection{Research context}

Having described some of the key motivations and rationale for this research, in what follows I introduce the key concepts and areas of the literature which have informed my approach, and which have provided a framework for discussing and 
analysing the material. I start with the broadest theme, imagination, and then get progressively more focussed in my discussion of various aspects of the research context. Although all of the themes discussed below have relevance for the thesis as a whole, some in particular are brought in or out of focus in each empirical chapter, depending on the case studies being discussed. For example, the theme of imagination is particularly relevant for chapter 5 , which discusses the role of art in socioecological imaginaries, while the second theme of utopia, dystopia, and the future is taken up in chapter 3, which looks at imaginaries of time and futurity. The third theme, stories, is broadly relevant across all of the empirical chapters, and the fourth, the Anthropocene, is particularly useful for understanding the context of chapter 4, which discusses approaches to human environment relations.

\subsubsection{Imagination}

As the previous discussion has indicated, I have approached this research from an assumption that imagination is an important dimension of socioecological transformation. However, imagination is a nebulous term and has been used in variety of ways, for example in terms of social imaginaries (Taylor 2004, Dawney 2011), technoscientific imaginaries (Marcus 1995), sociotechnical imaginaries (Jasanoff and Kim 2013), geographical imagination (Gregory 1994), and the geological imagination (Altena et al. 2015). The broadness of the term is part of its attraction, because it can be applied to a whole variety of aspects of life, and used to encapsulate how they come together to form our experiences of and in the world. However, this broadness also warrants a more detailed explanation of how I understand and use the term in this thesis.

Fundamentally, I approach the concept of imagination from an understanding that imaginaries are not opposed to "the real", nor are they a backdrop to the world, but, as Dawney $(2011,535)$ describes, "they are produced by bodies through practices and technologies and constitute the way in which we experience the world". In this sense, imaginaries are "central to an understanding of how bodies, individually and collectively, act on the world in order to manage affects, bring about change and in doing so produce subjects" (ibid.). For the purposes of this thesis I have predominantly approached imagination as a "social faculty" (Stoetzler and Yuval-Davis 2002, 325), which highlights how individual experience is situated in (and made possible by) a wider collective experience (Castoriadis 1994, Stoetzler and Yuval-Davis 2002). 
In addition, I am interested in plural imaginaries, rather than the idea of one particular social imaginary (Rossiter 2002). This is especially relevant in relation to climate change, where we find competing imaginaries formed from many intermingling sources (from scientific reports to disaster movies, for example) all of which inform a collective sense of "who we are and what we can become in times of climate change" (Sjögren 2016, 27). This is increasingly a position adopted in the literature, and in wider debates about climate (and other environmental) change, which argue not only that imagination matters for how societies respond to such issues, but also that it requires a concerted effort to understand and deconstruct current (ailing?) imaginaries of environmental futures in order to reclaim new ones and disrupt hegemonic imaginaries that can make it difficult to imagine alternatives (e.g. Hurley 2008, Yusoff and Gabrys 2011, Levitas 2013, Harris 2017b). Thus, Appadurai (1996, 4) sees imagination as a "space of contestation in which individuals and groups seek to annex the global into their own practices", and an important part of how "fields of possibility" (ibid. 31) delineate what people consider "possible and desirable to do, to know, to think, to feel, to dream at a certain point in time in a specific society" (Sjögren 2016, 26). Yusoff and Gabrys $(2011,516)$ also define imagination "as a way of seeing, sensing, thinking, and dreaming that creates the conditions for material interventions in, and political sensibilities of the world". This material and political potential of imaginaries for transformation is why I afford it central importance in this thesis as a way of approaching the various case studies and asking, essentially, what kinds of "economies of affect and imagination" (Rossiter 2002, 84) are being formed in relation to socioecological futures. Importantly, taking an interest in imaginaries also means developing an awareness of how the seemingly mundane or insignificant which might not be contested or seen as political are, in fact, part of imaginaries and their political potential (Sjögren 2016). As Latimer and Skeggs (2011, 393) contend, "imagination [is] one of the key sites in which all political and cultural agendas, large and small, are played out".

The concept of geographical imagination is particularly helpful for considering socioecological transformation, because it focusses on how ideas about space and place (and people's relationship with it) are shared, negotiated, consolidated, and reproduced in society in other words, how "habits of mind" about human environment relationships are formed (see Gregory 1994, Gregory et al. 2009). The question of how people imagine and render space can be thought of as crucial to socioecological transformation because it is about picturing the ways in which we inhabit the world an imaginary which can span past, present and future. As Massey (2013a) 
suggests, geographical imagination is a process and a way of knowing and making sense of the world, a way of encompassing the known and the unknown. In turn, these imaginaries can either limit or expand our perceived place in the world be that as individuals, communities, nations, or as a species. As Beck writes, the "cultural 'horizon' of people's expectations and values, born out of social experience and mediated via cultural symbols, history, and material conditions, must be the main point of reference for understanding ecological concern" (Beck 1995, 43). Thus, how we imagine our place(s) in the world influences how we behave in it (Harvey 1990).

Imagination is, therefore, not only an important concept for understanding how existing ideas and ways of perceiving space and place function in society, but also how new ones might arise and contribute to the formation of new socioecological conditions. However, it is increasingly suggested that there is a "crisis" in imagination when it comes to socioecological transformation (e.g. Buell 1996, Cheney 1999, Yusoff and Gabrys 2011, Wapner 2016, Magrane 2017). Harvey (2000) and Levitas (2013), for example, have written at length about a dearth of collective imagination that has resulted in a feeling that "there is no alternative" (Harvey 2000, 17) to social, political and economic conditions. More specifically, Wapner (2016), uses the idea of "Climate Inc." to describe a situation in which responses to climate change seem to be marked by a distinct lack of imagination, revolving only around narrow, hegemonic scientific and economic explanations about what is possible (see also Wainwright and Mann 2013). Such hegemony, as Harris (2017b) argues, closes down the imaginative space necessary to think otherwise about the climate crisis, and to create alternative ways of organising. This relates to Yusoff's (2009) suggestion, following Bataille, that approaches to climate change need to be able to incorporate dimensions of "nonknowledge" (of which imagination might be considered one), in order to go beyond the narrow accounts about climate produced through scientific data. Similarly, Braun (2015a) points out an opportunity and obligation for those in the social sciences to contribute to imagining socioecological transformation, rather than focussing solely on critiquing how we arrived at the current crisis (see also Chatterton et al. 2011). More broadly, Ingold (2013) suggests that acknowledging our imaginative participation in the more-than-human world will be crucial in any attempt to form more sustainable relationships with it.

In this sense, imagination presents both an opportunity (for novel forms of socioecological transformation) and a challenge (if, as the views outlined above suggest, there is a "crisis" in collectively imagining how the world could be otherwise) (Milkoreit 2016). This staging of imagination as an opportunity and a challenge is one 
which broadly underpins my approach to all of the empirical chapters in this thesis. However, chapter 5 engages most directly with the theme of imagination, with an exploration and discussion of the role of art in stimulating imaginative responses to environmental crises. Discussions about geographical imagination have frequently noted the importance of creative and aesthetic practices (see Harvey 1990, Gregory et al. 2009), but more recently this discussion has intensified around the role of aesthetics in socioecological transformation (e.g. Dixon et al. 2012, Hawkins et al. 2015b, Phillips 2015). The importance of aesthetic practices is in little doubt (as Castree, speaking at an event in 2013, contends, "imaginative works should be taken as seriously and afforded as much power as climate models and science with regards to their ability to shape the future" (Castree 2013b)), but there remains much to be learned and shared about the various forms that participation and engagement in art can take, and what role they have in reproducing particular imaginaries (Belfiore 2013). Chapter 5, therefore, contributes to these debates; it seeks to go beyond an instrumental engagement with "imagination" as something that can be provoked or manipulated by particular artworks and artists in response to environmental crises, instead developing an understanding of imagination and creativity as ways of participating in more-than-human worlds and, potentially, of transforming relationships with them.

\subsubsection{Utopia, dystopia, and the future}

Debates about imagination and socioecological transformation have been particularly directed at imaginations of the future; the future is conceptualised as a realm which is itself disclosed and related to in particular ways, and made present through particular practices (Anderson 2010). As Yusoff and Gabrys (2011, 519) contend, imagining environmental futures is part of how "possible futures are set in motion". Indeed, my original approach to this research was to explore future imaginaries in Wales, and although this remains an implicit intention, I have broadened my approach to encompass imaginaries more generally (as described above), because often it was very difficult to find or distinguish imaginaries and narratives which were explicitly about "the future". More often, I found that a whole host of discursive, representative and affective dimensions about the past, present, and the future, came together to form particular "economies of affect and imagination" (Rossiter 2002, 84) that, although not always explicitly about creating a "vision" for the future, still give clues as to how the future is desired to be and might become. Consequently, notions of the future, 
and future imaginaries, is a thread which runs through the whole thesis (although it is addressed most directly in chapter 3, which deals with imaginaries of time and futurity). In this section I will, therefore, review the broader context of work on future imaginaries within which I situate my own research.

I have started from an assumption that the future matters (Adam and Groves 2007); as Uprichard $(2011,2)$ summarises, "it matters in everyday life and it matters to the lives of everybody; it is an intrinsic part of the time and temporality in which all things are necessarily situated." As Anderson and Adey (2012) contend, there is a need to understand how geographies are made and remade in relation to the future, given that this geohistorical moment seems increasingly to be overshadowed by questions of what the future might be. Importantly, this approach to the future is not one that sees the future as already determined, or as a linear development from the past and the present, but as something that is actively and continuously being made. Given that futures are made present, or take on some form of presence, in a variety of ways, there is a need to question how relations between the past, present, and the future are achieved: "to understand how geographies are lived and made as futures are prophesied, imagined, deterred, regularised, invested in, hoped for and so on" (Anderson 2010, 778). Anderson (2010) also notes that human geographers have tended to neglect the category of the future compared with significant amounts of work on the past, memory, and haunting. In the seven years since his writing, work on futures has proliferated, particularly around notions of anticipation and anxiety (e.g. Neocleous 2012, Swyngedouw 2013a, Groves 2016), utopia (see below), ethics (e.g. Adam and Groves 2011), governance (e.g. Rickards et al. 2014), and affective dimensions of the future (e.g. Brigstocke 2016, Gerlach 2017b). However, there is a relative dearth of empirical work which engages with issues of how futures are, as Anderson says (2010, 778), "prophesied, imagined, deterred, regularised, invested in, [and] hoped for". This, perhaps, has something to do with the elusiveness of the subject the future, as a realm of imagination, might be easier to theorise about than it is to identify in empirical work, if only because methods for eliciting future imaginaries can be difficult to get right (Shirani et al. 2016). In the social sciences, efforts to do so have tended to focus on the future imaginaries of individuals (e.g. Schmidt et al. 1978, Hicks 1996, Tonn et al. 2006, Alm 2011, Lorenzoni and Hulme 2009). However, fewer studies have attempted to analyse future imaginaries which arise out of the socio-political context. Some examples include Fløttum et al. (2014), who analysed representations of the future in blogs about climate change; various analyses of future narratives in climate fiction and film (e.g. Hurley 2008, Whiteley 
et al. 2016), and of future technologies (Kinsley 2010); and narratives of future energy transitions (Miller et al. 2015). The research in this thesis therefore represents a novel attempt to bring together some of the theoretical developments around notions of the future (and imagination), with empirical examples in Wales. In doing so, the research also experiments with methods which have not previously been much associated with research on futures, such as ethnography and participant observation (this will be discussed further in chapter 2).

Given my concern with socioecological transformation, I have found the notion of utopia an important and helpful concept with which to think about future imaginaries. However, the concept of utopia (a word which "plays on the homophonic qualities of two Greek words عú (good) and oú (no, not) prefixed to a suitably

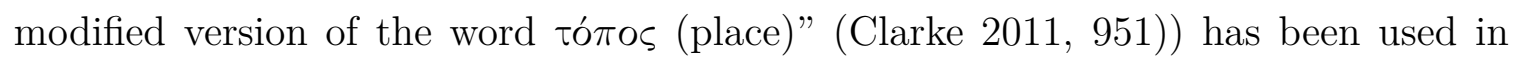
various ways. Kenny distinguishes between utopia deployed as (a) conceptions of an ideal society that are, nonetheless, not believed to be attainable; (b) calls for the creation of morally better societies; (c) detailed blueprints for the perfect societal future; and (d) various forms of 'social dreaming' that may help promote 'alternative' values and ideas (Kenny 2007, 212). Version (c) utopia as a detailed blueprint has been widely criticised for its associations with totalitarian political regimes, in the West and the East (Popper 1962, Berlin 2003, Gray 2007), and for the ways in which it closes down imaginaries of possible alternatives (e.g. Harvey 2000). Indeed, the notion of utopia continues to be popularly denigrated as "ideological" and "unrealistic"; as Kenny $(2007,212)$ puts it, the word utopian "dooms those to whom this label is attached to the status of the irrelevant, the marginal or the irrational". Nonetheless, a rich vein of work has continued to argue for the importance of utopianism as a kind of "social dreaming" (as in option (d), above) which urges and propels societies to transform. Perhaps the most famous amongst such commentators is Bloch, whose writings in The Principle of Hope (1986) defined utopia not as a pre-existing telos but as a process of utopic thinking that was driven by hope for a better future, and the anticipatory concept of the "not-yet". Bloch understood utopia as being continually built and emerging out of often overlooked expressions of hope in everyday life from fairytales to sport. Bloch's ideas about social change present a stark contrast to Freudian ideas of the time, because he understood hope for better futures as a more powerful driver of transformation than fear (generated by past events), which is what Freud identified as the more potent stimulus. Importantly, Bloch understood utopia not as a totalitarian vision, but as made up of fragmentary moments whose aesthetic quality allows them to be "utilized and reutilized for the realization of what has not- 
yet-become but can become" (Bloch 1988, xxiii). These formations can illuminate what is missing, but importantly they also instil hope and provide the impetus for individual and collective change (ibid.). More recently, critical social scientists have elaborated on the importance of this kind of utopian hope for the actualisation of socioecological change (e.g. Harvey 2000, Anderson 2006b, Anderson and Fenton 2008, Appadurai 2013). Levitas refers to such an approach as "utopia as method" (Levitas 2013, xviii), in which she understands utopia as a quest for grace, and for developing the necessary reflexivity and space for developing what (desirable) possible futures might be. Similarly, Jameson writes that "utopia as a form is not the representation of alternatives; it is the imperative to imagine them" (Jameson 2005, 416).

Nonetheless, there is a concurrent argument which many of the above-mentioned writers also express that utopianism is dying, or is already dead, and that intentional social change no longer seems possible (e.g. Bauman 2003, Featherstone and Miles 2014). The reasons for this down-turn in utopian thought range from Fukuyama's (2006) controversial suggestion that Western liberal democracies have reached "the end of history", and an end point in ideological evolution, to Bauman's argument that contemporary conditions of postmodernity are characterised, conversely, by an "unending sequence of new beginnings" (Bauman 2003, 11) in which utopianism cannot maintain a footing. As Clarke (2011, 952) writes (contra Fukuyama), "the end of utopia has not been brought about in the sense of it no longer being needed ... but because the conditions in which it could thrive have been liquidated." Indeed, Bauman's concept of Liquid Modernity (2000, 2007) is useful for contextualising current debates about utopianism. Here, Bauman identifies a belief in utopia as a defining aspect of modernity, and contends that the modern era was a "worldliving-towards-utopia" (Bauman 2007, 96). Utopianism, in this context, needed two conditions; first, a sense that the world is not functioning as it should, and, second, a confidence in human potency to rise to the task. In contrast, the fluidity of latemodernity, as Bauman describes it, means that change is so rapid that long-term visions and goals are rendered obsolete and replaced by preferences for flexibility and short-termism. In this situation, Bauman believes we have moved from a time when "utopia" meant a coveted, dreamt-of distant goal to which we could, should, and would, progress, to a time when "progress" no longer means shared improvement but, rather, individual survival a "desperate effort to stay in the race" (Bauman 2007, 104) in which a "desire for a better today has elbowed out concern with a better tomorrow" (Bauman 2003, 11). Similarly, Baudrillard (2000, 48) proposes that "life transformed" the goal of utopia has descended into "life-as-survival, which is a 
kind of minimal utopia". The prospect of environmental catastrophe only serves to further undermine any sense that the future will be better than the past. Clive Hamilton, in a recent article for Grist, sums up the situation thus:

What has died is our conception of the future, that has been around or been emerging since the industrial revolution: The idea that, whatever happens, we are moving into a brighter future where everything will be improving and getting better. And we are now facing a situation where that is a dangerous delusion. So, in that circumstance, what do we do? ${ }^{8}$

Hamilton describes a situation in which joyful utopianism seems inappropriate, but pure nihilism might not be so helpful either. With the loss of utopia "that peculiarly modern attempt to harness 'the urge to transcend"' (Clarke 2011, 952, citing Bauman 2003, 11) Baudrillard claims that there is a lack of "objects of belief. But even worse, perhaps, we lack objects in which not to believe" (Baudrillard 2000, 48). In lieu of utopia, it has been suggested (and, indeed, may be apparent to many of us) that a distinctly apocalyptic, dystopian tone has emerged (Skrimshire 2010, Žižek 2011, Featherstone and Miles 2014). Whether one takes the position that "dystopia is now" (Featherstone and Miles 2014, 128), or, as Clarke (2011) contends, that utopia has not failed but has in fact been achieved (in as much as it has succeeded in collapsing the modern divide between imagination and reality a distinction on which utopia depended to survive, thus rendering itself obsolete), there is a sense that the future is somehow "exhausted" and already determined (Brigstocke 2016, 92). While many contend that these conditions and visions of dystopia may be useful spurs and invitations for a rejuvenated utopian imaginary (e.g. Beck and Dorrian 2014, Claisse and Delvenne 2015, Featherstone and Miles 2014), there is also an argument that such crisis narratives serve only to perpetuate certain socio-economic-political arrangements (Klein 2007, Swyngedouw 2013a), legitimise anticipatory politics (Anderson 2010, Neocleous 2012), or simply cow people into positions of denial and apathy (e.g. Latour 2008, Beck 2010), with a sense that the future is already occupied, colonized, or destroyed (e.g. Hurley 2008, Brigstocke 2016). Conversely, it is suggested that utopia is lost precisely because the future cannot be visualised climate change, and the proposed Anthropocene, in particular, represent moments in human history with virtually no precedent and thus the risks and uncertainties seem incalculable and unknowable (Beck 1992, Chakrabarty 2009). As Anderson and Adey (2012, 1529) ask, "how to think a future that cannot be anticipated and exceeds all expectation"?

\footnotetext{
${ }^{8}$ Available at http://grist.org/climate-energy/is-the-anthropocene-a-world-of-hopeor-a-world-of-hurt/. Last accessed 7 November 2017.
} 
Clearly, the modern concept of utopia as intentional progress seems to be floundering. As Garforth et al. (2009, 3) contend, "as commitments to particular futures seem to recede or become impossible in conditions of late or liquid modernity, intention becomes increasingly problematic in attempts to hold onto hope and otherness." This opens up some interesting directions in which to take contemporary engagements with utopianism, especially in light of environmental crises. Garforth and Kraftl (2009) suggest that utopianism might be possible, or may occur, without the intentionality which has previously been assumed a defining aspect of utopia. Similarly to Bloch, they posit utopia as being as much about "otherness in the present" (Garforth and Kraftl 2009, 2), something identifiable in the most incidental and trivial aspects of social life, as it is about building better futures. Here, understanding utopianism means an awareness of how "any cultural formation includes within itself the possibility of an/other state of being" (ibid.), but these possibilities might be accidental, incidental or contingent, rather than something that is always and only intended. Garforth et al.'s provocation provides useful context for the research in this thesis, because I have included in my analysis both intentional utopias (such as the Welsh Government's visions for sustainable development ${ }^{9}$ ), and the kinds of incidental spaces of "otherness" (for example, as found in the practices of some of the other case studies) that do not necessarily explicitly envision a particular future, but which nonetheless contribute to the formation of one (see also Sargisson 1996, Fournier 2002). The research presented here therefore contributes, broadly, to questions about whether utopia can be, as Garforth et al. $(2009,2)$ put it "something that happens to us, rather than solely something we make, mean or intend." Such engagements with utopianism take something of a post-human turn, calling into question the primacy of intentional human agency in the making of futures (see also Gilbert and Lambert 2010). Nonetheless, I must also acknowledge that this thesis is itself a product of a certain "modernity", because it is concerned with the ways in which life might be transformed. The research is therefore situated within a wider body of work which tries to reconcile an undeniably utopian desire to contribute to socioecological transformation (Braun 2015a), while simultaneously avoiding some of the pitfalls of modernity which have, by and large, created the socioecological conditions of the present. In this, I follow Featherstone and Miles's reinterpretation of modernity, in which utopia is a mode of thinking about the ways in which the future, and the new, might emerge from a present which seems exhausted, while acknowledging that the ways it might do so may be diverse and not necessarily coherent. Theirs is a modernity which is not

\footnotetext{
${ }^{9}$ The Welsh Government's programmes are indications that, as Kenny (2007, 212) contends, proclamations of intentional utopia's demise have in fact been over simplified and over-stated.
} 
organized around core principles, such as scientific thought and progress through technology, or through the instrumentalism that undermined modernist architecture and urban planning. Instead, it is a generic, experimental, utopian form of modernity concerned with openness to the future and how we might extract the possibility of the new from the ruins of the present. But is this more than a salvage operation? Just as the liberating, progressive aspects of modern culture were trashed as some kind of state despotism and buried in the rubble of demolished towerblocks, so the hope that informed and, in many ways, shaped modernist culture remains a dynamic force for change. The first step, perhaps, is to reimagine the world and to reclaim the ways in which meanings in the world are produced, conveyed, and shared (Featherstone and Miles 2014, 129)

Other writers add that, for utopia to have any relevance or force in contemporary life, it will need to involve, amongst other things, thinking differently about what constitutes knowledge (Levitas 2013), experimenting with new possibilities for politics and political subjectivities (Braun 2015a), an attention to difference and plurality (e.g. Amin and Thrift 2005, Gibson-Graham 2008), and an openness to utopian possibilities which arise out of non-intentional, human and more-than-human assemblages (Garforth and Kraftl 2009). Finally, it will involve "thinking of space as animated by (im)possibilities, potentialities and virtualities" (Anderson and Fenton 2008, 78; see also Kraftl 2007). Given these provocations, and alongside the contribution this thesis makes to understanding how geographies are made and remade through the presence of future imaginaries more broadly (Anderson and Adey 2012), the thesis is also situated within these recent efforts to re-engage with the concept of utopia, and even to participate in making utopian spaces of heterogeneity and "otherness" more visible, more imaginable. Some methodological implications of such an approach will be discussed in more detail in the following chapter. However, alongside this commitment to engage with utopianism as spaces of heterogeneity and otherness, through the research I also found myself increasingly drawn to the benefits of adopting a dystopian imaginary once in a while. As I came to see it, the two are not necessarily contradictory, because it may be that a dystopian imaginary entails a processes of loss, grief, and acceptance that marks an important transition from a position of denial to that of transformation and adaptation. Thus, dystopianism can be thought of as an integral part of utopianism because, as Featherstone and Miles (2014) contend, it is only when we confront the worst, that we realise the urgency to invent 
the new. Similarly, as recent research published in Nature Climate Change points out, "ecological grief", is a legitimate and important (although under-acknowledged) mental health response to the unprecedented loss of biodiversity currently underway, and one which helps to remind us that climate change and ecological degradation are not just abstract scientific concepts (Cunsolo and Ellis 2018). I return to this subject in chapter 3. For now, I turn my attention to the final two themes of this section on research context; stories and the Anthropocene.

\subsubsection{Stories}

There seems to be an intuitive fit between storytelling and matters of imagination, the future, and transformation. Perhaps this is not surprising given that notions of transformation, and of the future, can be mapped easily onto a story-like structure of beginning/middle/end (or past/present/future). Indeed, as Cronon (1992) has argued, stories are integral to how thought tends to be organised and how experiences are ordered and made sense of. ${ }^{10}$ Stories also require imagination, and have the ability to encapsulate many different dimensions at once. As Cameron $(2012,574)$ observes (drawing on Polletta 2006),

stories embody a series of contradictions: they are understood as both authentic and subject to manipulation, idiosyncratic and signs of more general processes, disciplined by and exemplary of dominant narratives and yet capable of transgressing and transforming dominant narratives. Stories also demand interpretation; their normative, emotional, or moral effects are derived relationally, through interpretation, not directly conveyed. The concept of 'story' thus focuses attention on the problem of personal and collective experience and expression, and the ways in which modes of organizing, assembling, performing, and interpreting those experiences and expressions exceed the personal and particular.

In addition, "story" might be distinguished from "narrative" in that it offers "a kind of heterogeneity, materiality, sensuousness, and openness that narrative does not" (Cameron 2012, 577). And, while story commonly refers to a sequence of events, narrative can be considered as a discursive presentation of events (not necessarily in

\footnotetext{
${ }^{10}$ Although, it is worth pointing out that this doesn't necessarily apply to all cultures, particularly in the sense of assuming a consecutive progression of past, present and future. For example, in the South American Aymara language and culture, the future is conceptualised as behind, while the past is ahead (Núñez and Sweetser 2006).
} 
sequence) (Culler 1982, Phelan and Rabinowitz 2008). Thus, while a story implies a temporal element of transitioning from A to B to C, and so on, a narrative might be thought of more broadly as a discursive "style" a particular way of talking about or representing an issue (which need not necessarily entail a temporal "plotline"). Both stories and narratives are increasingly recognised as sites of interest for thinking about the workings of power, knowledge, and ideas, given their capacities to move, affect, and produce collectivities in ways which interweave the personal, social, structural and ideological (Cameron 2012, Haraway 2016). There has also been something of a "narrative turn" in the social sciences in response to dissatisfaction with the dominance of physical, technical representations of environmental issues (Moezzi et al. 2017). As Harris (2017a, 647) says, stories "matter in the traditional sense in that they are symbolic; they provide us with much needed motivation and orientation. However, they also matter in the sense that they animate the world around us; they carry with them material-discursive weight." While it is common to look for stories in traditional "story-telling" media, such as literature, film, and theatre (and, indeed, research is increasingly engaging with environmental stories and narratives in these contexts (e.g. Hurley 2008, Whiteley et al. 2016, Ghosh 2016)), stories and narratives are also woven through many aspects of life, for example, in policy, in conversations between people, in community projects, in curricula, and so on. Implicitly or explicitly, everything that is said and done arises out of, and contributes to, a particular telling of the world, or how it could be. Collard et al $(2015,327)$ therefore refer to these practices as "practices [that] bring worlds into being; different stories enact different worlds that may be co-emergent, partially connected, or in conflict." As Lejano et al. (2013, 2) contend, stories and narratives are also how "people both analyze and realize personal relationships with land, animals, rivers, air, and even bacteria."

From this perspective, it is possible to see why stories and narratives might be so important in relation to socioecological transformation, for they engage people's capacities individually and collectively to speak into existence alternative worlds and ontologies (Blaser 2010). Consequently, they "matter", not only in the sense that they create meaning and are meaningful in people's lives, but also in the sense of the "mattering" effect of stories; experiences of reality are created through the discursive privileging and processing of certain materials, and thus stories might be considered just as agential in relation to environmental crises as humans, rocks, and $\mathrm{CO}_{2}$, for example (Barad 2003, Harris 2017b). Stories are, in one sense, representations, but they can also be useful for thinking about how the more-than-representational world, 
that is, the affective, experiential aspects of life (Cameron 2012), come to shape our "storylines" about the world even if they remain unwritten or unspoken. Stories can therefore be a helpful conceptual bridge between a humanistic emphasis on representation, discourse, and meaning, and the more material, corporeal approaches of non-representational, post-human branches of critical geography (Saldanha 2012). This seems particularly relevant in relation to imaginations of the future, which, as Adam and Grove $(2007,201)$ contend, is an "invisible reality" which is constituted by and through "atmospheres" of emotion, intuition, and affect, the known and the unknown (Anderson 2009).

Some stories, however, are valued and told more than others, and this is a crucial point. Given that, as Barad contends, discourse is not only what is said, "it is that which constrains and enables what can be said" (Barad 2003, 819, emphasis added; see also Foucault 2002), which stories come to matter has important implications for how environmental crises are responded to. Climate change and the Anthropocene (more on which later) are concepts that pose enormous imaginative challenges, particularly in traditions of Western thought, because they overturn dualistic ideas about humans' separation from the environment, indicating, instead, how fundamentally entangled and interdependent human and more-than-human agencies are, and how these relationships play out over vastly longer timescales than had previously been imagined. Latour, referring to the oft-repeated warning that humans have breached several "planetary boundaries" (see Rockström et al. 2009), sums up the predicament thus:

$[\mathrm{N}]$ ot only do we have to swallow the news that our very recent development has modified a state of affairs that is vastly older than the very existence of the human race [...] but we have also to absorb the disturbing fact that the drama has been completed and that the main revolutionary event is behind us, since we have already crossed a few of the nine "planetary boundaries" considered by some scientists as the ultimate barrier not to overstep! [...] How can we simultaneously be part of such a long history, have such an important influence, and yet be so late in realizing what has happened and so utterly impotent in our attempts to fix it? (Latour 2014, 1)

Latour contends that this perplexing state of affairs is one for which people are not sufficiently mentally or emotionally prepared (at least, within the context of Western modernity that Latour writes), and he asks, "how do we tell such a story?" (Latour 
$2014,3)$. If, indeed, this moment in history represents a pivotal time for constructing new stories and narratives about how to live in the knowledge of such crises, and for responding to them (the scientific consensus also seems to indicate that the next few decades represent a brief window of opportunity to minimize the potentially catastrophic effects of climate change (e.g. Pachauri et al. 2014, Clark et al. 2016)), then it is vital to investigate how, why, and from where stories about these situations and how to change them are emerging. ${ }^{11}$ Ghosh (2016) suggests that the task is, therefore, not to focus on the inevitability of particular environmental futures, but rather on the issue of which stories get told and valued, and how they shape our current predicaments. Indeed, a key provocation for this thesis has been Castree's (2013b, unpaginated) contention that, as we cannot directly experience the future, we can only be affected by conceptions and images, imaginations, representations of what is to come. Thus, "whose mirrors and which magnifying glasses" we use is a key consideration for research in this field.

I have, therefore, found story and narrative helpful concepts for approaching, analysing and discussing imaginaries about socioecological transformation in Wales. In particular, the Welsh Government's policy innovations lend themselves to this approach. Environmental policy is, as Harris (2017b) contends, a major character in larger climate/environmental stories. Thus, policy shapes discourse and determines which parts of the story can be told, and policy itself can be understood as a kind of narrative (Roe 1994). However, story and narrative have also been useful ways to approach the other case studies in this research, for example, those which might be thought to constitute the more informal, "smaller stories" (Lorimer 2003, 197) of socioecological transformation which "narrate themselves into existence" (Lejano et al. 2013, xii) Ingram et al. 2014). Focussing on these smaller stories can be a way, as Lorimer (2003, 200) suggests, of "pausing to account for particularity, not in opposition to the general, but as a way of chafing against the ways in which the particular tends to figure in 'grand, scholarly stories"'. Or, put differently, stories disclose how "it is possible to find small kingdoms of worldliness, and to craft short stories as outcrops of global history" (Lorimer 2009, 269). To focus on these stories is therefore to recognise that local storytelling can also be sites of political transformation and disruption (Cameron 2012, Kolinjivadi et al. 2017), indicative of the "bottom up" nature of some socioecological change (Loftus 2009).

\footnotetext{
${ }^{11}$ This is not to say, as with utopianism, that stories necessarily have to be intentional; stories can also be a useful approach to thinking about how collective experiences arise unintentionally, and from human and more-than-human interactions (e.g. Lorimer 2006, Kohn 2013, Ingram et al. 2014).
} 
Cameron (2012) describes how these two approaches to storying one which sees stories as "metanarratives" for understanding power and knowledge, the other which seeks to apprehend the small without immediate recourse to larger explanatory frames might be seen as contradictory and in tension with one another. Indeed, she shows how a recent surge in interest in stories of the small, the local, and the particular has occurred, in part, as a relational and material turn against existing engagements with storying which were predominantly concerned with the ways that power and knowledge operate, but which it has been argued overlook the more intimate, non-representational aspects of life. On the contrary, I find storying to be a useful analytical concept precisely because it facilitates an attention to both, and, moreover, because the broadness and complexity encompassed by the concept of "story" helps to blur the boundaries between scales (Marston et al. 2005), between representational and non-representational, and between knowledge and emotion. As Cameron (2012) acknowledges, power and the political are not only about what we say, know, and represent, but also emerge from the felt, embodied, and ontological. Storying can take the form of a "metanarrative" which references a kind of transcendental morality (as with some political narratives), but storying can also be orientated "toward the emergent, the not-yet-here, [participating] in the materialization of new realities" (Cameron 2012, 580). It is in this sense that I refer to the "margins and the mainstream" in the title of this thesis. Following Katz (1995; 1996), I do not use these words as substitutes for "unimportant" and "important" (respectively), but instead I seek to take the marginal seriously as a form of "minor theory" that might present alternative ways of seeing things. As Katz contends, the "minor" is not oppositional to the "major", but it may subvert it from within (Katz 1996, 489), offering a flexible means "for thinking about practice in new and revitalized ways" (Katz 1996, 494).

To summarise, I understand stories to be important in relation to socioecological transformation because stories are a component of personal and political mobilisation, and a way of producing meaning and change (Gibson-Graham 2008, Bochner 2012). Stories can be understood as both as symptoms of dominant discourses, and as integral to the creation of alternative worlds, enabling subjects to inhabit these new discursive terrains (Gibson-Graham 2008, Haraway 2008). Stories represent but they do not only represent; they also affect and move, they are "inclinations that draw us places" (Cameron 2012, 584, citing Rose 2006). To this end, I have treated the case studies in this thesis as sites of stories and narratives from which to learn something of how socioecological transformation from the margins to the mainstream is imagined and enacted. 


\subsubsection{The Anthropocene}

The Anthropocene is possibly one of the most audacious stories there is about socioecological transformation. Latour $(2014,3)$ has called it a "geostory", and Castree (2014b, 468) remarks that the proposed re-naming of the current geological epoch is "an extraordinary act of representation", a neologism that has the potential to become central to everyday discourse, embedded in the "story-lines" about the way the world is, or should be (Hajer 1995, 56). In this section I summarise why the Anthropocene is considered such a significant proposal and one in need of critical reflection and how debates about it have informed my approach to this research.

The Anthropocene has been proposed as a new geological epoch (Crutzen and Stoermer 2000, Crutzen 2002), recognisable by the trace that the human species will have left in the geological record, thus making this era "functionally and stratigraphically distinct from the Holocene" (Waters et al. 2016, 137). Numerous proposals have been put forward as to when the Anthropocene "begins", ranging from the early spread of agriculture, to the first nuclear detonation (Lewis and Maslin 2015). Despite the Anthropocene not yet having been formally adopted as a geological epoch by the International Commission on Stratigraphy (ICS), the concept has attracted significant attention and captured imaginations, from coverage in the The Economist, National Geographic, The New York Times, and the New Yorker, for example (see Castree 2014a), to numerous journals and books bearing its name (e.g. Ehlers and Krafft 2006, Davis and Turpin 2015, Lorimer 2015, Scranton 2015). And although the Anthropocene is still largely debated as a matter of scientific fact (e.g. Zalasiewicz et al. 2017), the concept itself has huge discursive implications and, accordingly, is becoming the site of much critical scrutiny (e.g. Castree 2014c, Latour 2014, Malm and Hornborg 2014, Yusoff 2013).

Much of this discursive power, as Chakrabarty (2009) points out, has to do with how the Anthropocene concept invites people to imagine humans' impact on the planet on a geological scale; "to call humans geological agents is to scale up our imagination of the human", Chakrabarty says $(2009,206)$, adding, "we humans never experienced ourselves as a species ... [t]he discussion about the crisis of climate change can thus produce affect and knowledge about collective human pasts and futures that work at the limits of historical understanding" (ibid., 220 221). The Anthropocene concept invokes new conceptions of time (Nordblad 2017, Strydom 2017), agency (Latour 2014), knowledge (Johnson et al. 2014) and governance (Dalby 2013, Clark 2017, Yusoff 2017), and presents an opening for cultural and political reimaginings of 
human relations with the more-than-human world (Delanty and Mota 2017). Some have suggested that this is a positive development, signifying a new-found awareness of the inseparability of human and natural histories. For example, Clark and Yusoff's appraisals of the ontological implications of the Anthropocene tend to be hopeful ones, pointing the way to an understanding of humans as thoroughly earthly creatures not only fleshy, biological ones, but geological ones, too (Yusoff 2013, Clark 2017, Clark and Gunaratnam 2017). Similarly, Gibson-Graham and Roelvink (2010, 322) suggest that the advent of the Anthropocene might inspire an openness to conceptions of the world that is "a living process of inter-being ... a process of co-constitution that produces a new body-world". Others look to the possibilities for creating a "good" Anthropocene, replete with opportunities for creating a more habitable and welcoming epoch (Buck 2015), not only for humans but for other species, too (Collard et al. 2015).

Others have greeted the proposal of the Anthropocene with a great deal more hostility. For example, Malm and Hornborg (2014), amongst many others, question the use of the species category "Anthropos" and the ways in which this obscures intraspecies inequalities which are part and parcel of current ecological crises. They also contend that the Anthropocene narrative also returns us to some humanistic ideas, as humanity is portrayed as "a species ascending to power over the rest of the Earth System" (Malm and Hornborg 2014, 62). Similarly, Anderson (2014b, 13) recognises an emerging paradox, whereby, while "the threat of ecological catastrophe is provoking a critique of the idea that humans exist apart from nature, this threat also appears to be prompting a renewed commitment to the idea that humans possess a unique capacity to control our environment." Crist $(2013,129)$ adds that the discourse of the Anthropocene "refuses to challenge human domination", facilitating a perception of the world that is tenaciously anthropocentric. There is also a concern that the prefix "Anthropos" implies that all humans are the cause, and that there is something intrinsically bad about humans. Not only does this, as Malm and Hornborg (2014) say, obscure the vast inequalities between different human communities (and their vastly differing ways of perceiving and interacting with the environment), but it also shifts attention from what some argue to be the real culprit of environmental change, which is not humans per se, but one particular way of organising that is, industrial (and now, increasingly, neoliberal) capitalism. The focus on "humans" as a singular category, it has been argued, therefore perpetuates racialised and neo-colonial discourses about, for example, population (e.g. Saldanha 2013, Malm and Hornborg 2014, Last 2015), whereas the attention should really be on the resource-intensive lifestyles (and 
economies) of the relatively few. Elsewhere, it is suggested that the Anthropocene is increasingly being deployed as a way of generating future-oriented anxieties which are subsequently used to legitimise reactionary bio-politics such as increased surveillance and securitisation (Evans and Reid 2014).

Other names have been suggested as more appropriate for this era; the "Capitolocene" (Moore 2017, 2015), for example, or Haraway's "Chthulucene", which seeks to name the myriad entanglements of "temporalities and spatialities and myriad intra-active entities-in-assemblages including the more-than-human, other-than-human, inhuman, and human-as-humus" (Haraway 2015, 160). However, the Anthropocene still appears to be the most prominent name, gradually gaining acceptance as the signifier of the current geological epoch. Indeed, it is already much more than simply a name for a geological epoch, having become "a contentious term and a lightning rod for political and philosophical arguments about what needs to be done, the future of humanity, the potential of technology and the prospects for civilization" (Dalby 2015, 34). And yet, as Johnson et al. (2014) contend, "the Anthropocene" remains a relatively roughly-defined place-holder for an uncertain and unprecedented historical condition. Particular scrutiny is therefore warranted regarding the ways in which the term (and associated concepts such as Planetary Boundaries (Rockström et al. 2009)) is used, as well as of the multiple tropes, metaphors, visions, and knowledges that it sets, and that set it, in motion. Given how the concept of the Anthropocene seems to cross-cut so many important scientific, philosophical, ethical, and political issues, how it is interpreted, and who gets to invoke which framing of it, matters greatly, as Dalby $(2015,33)$ says, "for the planet and for particular parts of humanity". If the Anthropocene emerges as a dominant framing for talking about socioecological transformation, than it is also vital to ask "who is dominating the conversation about how to change the state of things?" (Todd 2014, 244).

The Anthropocene concept is perhaps best thought of as an imaginative "spark" (Collard et al. 2015, 326), a touch paper for transforming how humans perceive their place(s) on earth, although it has also been suggested that the concept is in many ways too huge to imagine. ${ }^{12}$ Either way, it seems clear that the Anthropocene is an imaginative issue as much as it is a matter of mere designation decided by the International Commission on Stratigraphy. Indicative of this, the Anthropocene is increasingly being engaged with in terms of narrative and story (see Veland and Lynch

\footnotetext{
${ }^{12}$ Morton $(2007,2013)$ describes the Anthropocene as a "hyperobject", an entity that signals such vast temporal and spatial scales that it defies traditional ways of understanding the world, and which is simultaneously ubiquitous and elusive, notoriously ungraspable.
} 
2016, Bonneuil and Fressoz 2016, Kunnas 2017), because such an approach helps to highlight how the imaginative framing of the Anthropocene has the power to shape thinking, emotion, and action, and because it has a familiar story-like structure of a beginning, middle and end, although what these will look like (or be defined as) is precisely what is at stake. In addition, approaching the Anthropocene from the perspective of storying recognises that there are, and will be, multiple ways of telling the story, each with varying ethical and political repercussions. Accordingly, engaging in the "anticipatory semantics" of the Anthropocene is necessary in order to fill in its contours (Johnson et al. 2014, 2).

The research presented in this thesis therefore contributes to ongoing efforts to critically examine the ways in which the idea of the "Anthropocene" emerges. Although none of the case studies I have engaged with explicitly refer to the Anthropocene, their practices and stories might be considered as indications as to how this particular geohistorical moment will or could unfold, and the forms that socioecological transformation "in the Anthropocene" might take. As Castree (2014a, 443) has noted in reference to recent apocalyptic Hollywood films, often the Anthropocene concept appears in "all but name". This is particularly relevant for the Welsh Government's approach to socioecological transformation, where the influence of Global Environmental Change research, and the concept of Planetary Boundaries both of which are closely related to the Anthropocene concept are present, and which suggest that Anthropocene idea is beginning to shape policy approaches even if it is not referred to by name. Dalby (2013) argues that critical scrutiny of these emerging geopolitical discourses in light of the Anthropocene will be essential. In addition, and as noted previously, the thesis as a whole is guided by calls for critical social science to play a more active role in conversations about environmental change, and the Anthropocene is fast becoming a central part of this conversation (see Castree 2014a, 2014c, 2014b). As such, each of the following empirical chapters which discuss conceptions of time, of human-environment relations, and art (respectively) can all be interpreted in light of the Anthropocene concept, although it has not been my intention to "force" this framing upon them.

\subsection{Research questions}

This study focusses on the socioecological imaginaries of several organisations based in Wales (which will be introduced in the following chapter). I have approached 
each of these as potential sites and examples of socioecological transformation, an approach which is inspired by Gibson-Graham and Roelvink's (2010) suggestion that, rather than asking the time-honoured but often paralysing question of "what is to be done?", perhaps there is something to be learned from asking "what is already being done?" (Gibson-Graham and Roelvink 2010, 331). Within this broad aim, a number of questions have guided my approach:

- How do assumptions about the future, and temporality, implicitly or explicitly shape imaginaries of socioecological transformation?

- How is socioecological transformation envisaged, experienced, narrated, and hoped for?

- What kinds of human environment relations are being imagined and performed, and how is the more-than-human world made present?

- What are the "affective lives" of the ideas being produced and reproduced, and what kinds of socioecological futures do they make possible?

The study is thus a critical analysis of socioecological imaginaries that seeks to challenge taken-for-granted or deterministic ideas about what the future will be, or what socioecological transformation entails. In this respect, it responds to calls for better understandings of how societies construct socioecological futures (Appadurai 2013), and how geographies are made through, and in relation to, socioecological crises (e.g. Anderson 2010, Anderson and Adey 2012). By deconstructing dominant framings and also by maintaining an attentiveness to the ethical and political possibilities emanating from the "smaller stories" of socioecological transformation, the research presented in this thesis represents a novel study of socioecological imaginaries in Wales, a country whose political innovations make it an interesting and important site for the research, but which has so far received little critical scrutiny.

On a more personal level, this thesis also represents my own story of learning how to think about socioecological crises, what possibilities for transformation might be, and how I might relate to them. The research has, therefore, been an intervention into my own "mental ecology" (Guattari [1989] 2014, 12), as the ideas, people, and practices that I have encountered along the way have produced innumerable, and often radical, shifts in how I think about the world, and how it might be otherwise. 


\subsection{Thesis structure}

From here, I develop the theoretical basis for the thesis with a chapter on my methods and approach. After that, three empirical chapters form the body of the thesis, followed by a short concluding chapter (chapter 6). Each empirical chapter is relatively stand-alone, containing relevant reviews of literature, analysis and discussion. The theme for each chapter was not predetermined but, rather, developed out of the research itself. The first of these (chapter 3) explores ideas about time and futurity, comparing and contrasting these across various case studies. The second empirical chapter (chapter 4) then shifts focus to more spatial imaginaries, to examine how relations between humans and environment are narrated and performed; the final empirical chapter (chapter 5) homes in on a more specific topic, that of the role of art in socioecological transformation. 
CHAPTER 1. 


\section{Chapter 2}

\section{Ways of knowing, ways of working}

There is always a story to how thought occurs (Rose 2016, 137)

In this chapter I describe how my epistemological and ontological approach to the research relates to my aims and intentions, my choice of case studies, and my choice and use of various methods. Along the way I consider some of the challenges and "blind spots" inherent in particular philosophical approaches and methods, and some difficulties that I encountered.

\subsection{Ways of knowing}

My perspective on the "work" that research does has changed considerably during the course of this $\mathrm{PhD}$. At the outset, and after undergraduate and MSc training predominantly in environmental sciences, I approached human geography (a relatively new field for me) with a similarly "scientific" aim: to find out about representations of environmental futures in Wales, analyse them, and write about them. I envisaged that I would, through the research, be able to represent various themes and then make legitimate claims about the general population based on these. I hoped that my "findings" would then be disseminated to interested parties, such as the Welsh Government or non-governmental organisations working on sustainability, for example.

These ambitions stemmed from an idea of myself as a detached observer, but it was an idea that quickly dissolved as my research, and reading, progressed. Fieldwork is intensely personal (England 1994), and there is no getting away from the fact that 
the words and ideas that make up this thesis are fundamentally shaped by the interactions of my own history, background, education, knowledge and ideas with those of the people and places I have encountered during the course of the research. As I began to meet research participants, join in with their activities, and learn about their ideas, I quickly became embroiled, and could no longer claim to be a detached observer. Not only did this steer me away from a morally unjustifiable stance of treating participants as mere "mines" of information (Stanley and Wise 1993, 168), but it also brought the research process alive and steered me towards some unexpected outcomes of this research: shared ideas, friendships, and experiences. As such, I feel that whatever I convey in this thesis should not been read as a representation of the world(s) I have been researching, but rather constitutes my response to my encounters and interactions with them (Rose 2016, 143). Although such an autobiographical approach can be derided for being "soft" and "touchy-feely" (see Crang 2003), elsewhere bodily and affective engagements are increasingly acknowledged as important realms of experience, knowledge, and politics (e.g. Dewsbury 2010, Thrift 2011, Hawkins 2013). This is particularly important in relation to socioecological transformation, where the "molecular domains of sensibility, intelligence and desire" are considered to be crucial (Guattari [1989] 2014, 18). An autobiographical approach is therefore well-positioned to capture some of the more intangible and embodied elements of experience and encounters with different ideas and imaginaries (Bateson 1979). Such an approach also corresponds to moves within geography to understand research as a process of learning through the body's own responses and "haptic knowledges" (Crang 2003, 499). It also acknowledges that these experiences are highly variable between people (Flowers et al. 2014, Haraway 1988).

The aims of this research have, as outlined in the previous chapter, been guided by my own particular ethical-political perspective on what constitute ecologically "good" (and bad) ideas. While such partiality is often considered a "nuisance" in research, something to be accounted for and the effects of which to be diminished as much as possible (see Silverman 2013), in some areas of research (particularly in geography), the ethical-political convictions of the researcher are considered central to the aims of conducting the research in the first place. This marks a radical shift from the view of researcher as detached observer to that of the "activist" academic or critical social scientist (e.g. Castree et al. 2010) who seeks to use their academic work to further particular political, social or ethical causes either theoretically or practically or both usually in line with the values and ideals of their research participants. Haraway's $(1994,62)$ view, for example, is that the researcher's role is not only to 
read and analyse worlds, but also "to get at how worlds are made and unmade, in order to participate in the processes, in order to foster some forms of life and not others." Thus "the point is, in short, to make a difference however modestly, however partially, however much without either narrative or scientific guarantees." (ibid.).

Acknowledging the subjective nature of social research has also been a process of accepting that I cannot directly speak for other people. As England (1994, 251) explains, "what I will be studying is a world that is already interpreted by people who are living their lives in it and my research would be an account of the betweenness of their world and mine." She stresses that the researcher can never pretend to fully represent their informants' voices, and that research arises precisely in this "betweenness" between their worlds and our own. In order to accept responsibility for the research we produce, England believes the first step is to expose the partiality of our perspectives, for to "speak for them and not myself" (ibid.) is morally unjustifiable. The purpose of this chapter is therefore to give a detailed impression of what the partiality of my perspective is, and how it has guided my approach to the research.

\subsubsection{Utopia as (my) method}

One of the first books I picked up at the start of my $\mathrm{PhD}$ was Levitas's Utopia as Method (2013), and it struck a chord. During my MSc studies I had become more and more interested in the notion that "doom and gloom" warnings about environmental catastrophe were not only ineffective in stimulating personal and social change, but that they might be detrimental. I found inspiration in popular books like Do good lives have to cost the Earth? (Simms and Smith 2008), which traded on the conviction, as earlier expressed by Bloch (1986), that utopic thinking is driven by hope and the possibility that things might, or could be, better in the future. Levitas, also influenced by Bloch, developed the notion of utopia into a reflexive method for the 21st century that, she argues, is both appropriate and necessary. It is worth quoting her at length here, as her proposal has been fundamental to my own approach to research:

[a utopian method] provides a critical tool for exposing the limitations of current policy discourse about economic growth and ecological sustainability. It facilitates genuinely holistic thinking about possible futures, combined with reflexivity, provisionality and democratic engagement with 
the principles and practices of those futures. And it requires us to think about our conceptions of human needs and human flourishing in those possible futures. The core of utopia is the desire for being otherwise, individually and collectively, subjectively and objectively. Its expressions explore and bring to debate the potential contents and contexts of human flourishing. It is thus better understood as a method than a goal a method elaborated here as the Imaginary Reconstitution of Society. (Levitas 2013, xi)

Such reconstitution, as I outlined in the introductory chapter, is a pressing need (although I would add to Levitas's proposal the need to consider all life, not only humans), and corresponds with Haraway's (1994) suggestion that the researcher can and should participate in making worlds rather than just observing them. Feminist political-economists Gibson-Graham have been central to carving out new spaces and roles for the researcher in this vein. They emphasise how, in focusing on some ideas and narratives rather than others, the researcher participates in the materialisation of new realities, alternative worlds, and possible futures. In bringing marginalised activities to light, for example, researchers have a role to play in helping to make such activities more "real and credible as objects of policy and activism" (GibsonGraham 2008, 613). Like Levitas, Gibson-Graham have been especially interested in opening up "an imaginative space for ... alternatives at a point when they seemed to be entirely absent" (Gibson-Graham 2008, 613).

The utopian and ethical stance taken by writers such as Levitas and Gibson-Graham has greatly influenced my approach to selecting case studies. I have chosen to focus on case studies which at least at the outset respond to current socioecological problems in either novel, or "marginal" (i.e. not mainstream) ways, and which propose to help life flourish. They suggest ways of living, doing and organising which, in some way or other, represent an imaginary reconstitution of society and of ecologies of ideas. In taking such a focus, I have necessarily averted my gaze away from "nonutopian" narratives, practices, and ideas which may, nonetheless, have important implications for socioecological imaginaries. I have chosen not, for example, to focus on dystopian fiction and cinema because I am inspired by the sentiment, expressed by Williams $(1989,118)$, that "to be truly radical is to make hope possible, rather than despair convincing". Gibson-Graham (2012 37) ask "what if we were to accept that the goal of thinking is not to extend knowledge by confirming what we already know, that the world is a place of domination and oppression? What if instead we thought about openings and strategic possibilities in the cracks?". This is not to 
say that it isn't important to, as Levitas (2013, xi) says, "expose the limitations" of current practices and discourses, but it is to recognise that, in addition, perhaps researchers have a responsibility to seek change, "for unless we begin to believe in the possibility of change and begin to encourage the marginal (and even that which does not yet exist, but could) by actively storying these margins, [then] change will not occur" (Cameron 2012, 581). Adopting utopia as a method, Levitas writes, "involves both making explicit the kinds of society implied in existing political programmes and constructing alternatives. It entails also considering the kinds of people we want to become and that different forms of society will promote or inhibit" (Levitas 2013, xviii).

A "hopeful ethics" (Chatterton et al. 2011, Gillan and Pickerill 2012) also relates to calls for a re-enchantment of geography in general (e.g. Woodyer and Geoghegan 2013). Enchantment is a "reparative" approach to research, which seeks to direct attention from "repression and oppression to possibilities and potentialities" (Woodyer and Geoghegan 2013, 197). Bennett (2001), contends that there is in fact a strong ethical case for enchantment, and insists that it is by no means a redundant concept in an era where dystopia often seems to rule in fact, she suggests that (re)enchantment is perhaps one of the most important political projects of our times. In a similar vein, Latour has argued that critical thought can be misused, at risk of fuelling atmospheres of distrust, ambivalence, and conspiracy, and he suggests instead that the academy needs to turn its attention from "matters of fact" to "matters of concern", and in doing so endeavour not so much to "debunk" but to protect and care (Latour 2004b, 232). Enchanted geographies might also be thought of as a response to colonial academic narratives in the West which have been tied to a "disenchantment of the world" (Weber [1948] 2009), in which imagination is subordinated to instrumental reason and rationality. Herman argues that such an obsession with rationality and the disenchantment it gives rise to constitute "epistemological blinders [that] border on ideology and pose an an enormous obstacle for geography" (Herman 2008, 74). From this perspective, hopeful ethics and enchantment therefore play a crucial role in widening geography's view and its participation in how worlds are "made and unmade" (Haraway 1994, 62).

While for some a hopeful, enchanted, orientation might seem "pollyanna-ish" (see Gibson-Graham 2002, 25), others argue for the political potential of hope (e.g. Anderson 2006b, Blomley 2007, Sparke 2007). However, being hopeful, or utopian, does not necessarily mean abandoning any attempts to interrogate problematic practices and ideas; I found that while, out the outset, some case studies appeared to offer hope- 
ful, transformative alternatives to the status quo, during the course of the research it became apparent to me that often these cases were underpinned by problematic ideas and narratives which I felt warranted a more sceptical approach. It is for this reason that, while in general my stance has been a hopeful-utopian one, I bear in mind Chatterton et al.'s call for research projects in this vein to be "hopeful but not naively so. They are often normative, probing 'what ought to be' rather than just 'what is': in this sense, they may be explanatory-diagnostic but also anticipatory-utopian. They may interrogate wider structural logics but also be based in lived experiences" (Chatterton et al. 2011, 185). Thus, I have tried to balance Gibson-Graham's (2012) approach of bringing to light practices at the margins, with a simultaneous effort to reflect on how dominant framings of socioecoloigcal transformation could be different (England 1994). I think Haraway $(1992,326)$ sums this orientation up best by suggesting that it is a matter of being both generous and suspicious.

\subsubsection{Analysis through stories, narratives, and "Earth-writing"}

Telling ought never be thought of as an easy option or an innocent act. (Lorimer and Parr 2014, 543)

As introduced in the previous chapter, I have approached the research from a perspective of stories and narratives, essentially asking "what story is being told here?" Narrative analysis is an approach which attempts to identify the basic story being told, focussing on the way a narrative is constructed, the intention of the teller and and the meaning of the "plot" (Riessman 1993). Relatedly, discourse analysis is concerned with how knowledge is produced through the use of language, or through the adoption of implicit theories and ideas, and how these discourses are both produced by and producers of material practices and power relations (Ritchie et al. 2013, Feindt and Oels 2005). Coming back to Bateson and Guattari's emphasis on the importance of ideas, and the suggestion that "the ecological ideas implicit in plans are more important than the plans themselves" (Bateson 1972, 513), stories and narratives can also be though of as kinds of "vehicles" for particular ideas and concepts.

Accordingly, I have adopted a broadly poststructural approach to analysis which has an eye towards the histories and social contexts of the ideas which constitute particular narratives (e.g. Derrida 1967, Foucault 2002), and which is wary of trying to "pin down" meaning in any precise, stable, or essential way, but rather understands "reality" as produced and reproduced through discourse. However, Cronon asserts that 
there is a tension between the "endless postmodernist deconstruction of texts that fails to ground itself in history, in community, in politics", and in the practicalities of addressing the "moral problem of living on earth." He implies that the postmodern/poststructuralist claim that "there is no outside-text" (Derrida 1977, 144) leads to a seemingly existential and hopeless position. On the contrary, it seems to me that there is a degree of crossover between Cronon's $(1992,1374)$ assertion that narrative is "our best and most compelling tool for searching out meaning in a conflicted and contradictory world", and the poststructuralist conviction about the groundlessness of texts and narratives is what affords them a degree of play, creativity, and openness to be interpreted in a variety of ways which, in turn, influence how we experience the world (Barthes 1972, Silverman 2010). If indeed, "stories are all we are" (King 2003, 1), as a researcher I feel there is, then, an imperative to embrace and work with narratives and stories as ways of participating in "making worlds" (Haraway 1994, 62 ), not only by representing them but also in responding to them. In responding, I myself am involved in a process of narrating the people, places and stories I have encountered, of weaving them together in this thesis. It would be impossible for me to list chronologically (or even to remember or record) all of the elements which have influenced this thesis over the past four years, and as such I have necessarily chosen to include some things and not others, and I have determined where it should "begin" and "end" (Cronon 1992, 1354). As such, what is written in these pages is not a complete account nothing ever can be but a story, a version.

As Cameron (2012, 586) writes, "from a poststructuralist perspective, geographers have always 'told stories'; if one accepts the proposition that knowledge is narratively constructed then all geographic writing must be understood as a form of storytelling". Indeed, the word "geography" comes from the ancient Greek verb geo-graphein, meaning "to write" or "to scratch" the earth. In essence, this description sees the geographer's role as akin to that of a story-teller "in interpreting the earth, geographers inscribe its surface with narratives and stories" (della Dora 2015, 225). Rose (2016) elaborates on the value of stories in terms of how they, and we (as researchers), "inscribe" the earth. He highlights how, through stories, researchers can reposition themselves in relation to the topic of research, and to the research participants. He argues that stories are more than "just" evidence, and he distinguishes between empirics and stories. Whereas the purpose of empirics is to evidence a theory, the work of stories is to "weave together and order the [author's] confused and uninformed conception of temporal life" (Rose 2016, 134). The risk associated with empirics and theories, Rose notes, is that using one to prove the other makes the research partic- 
ipant "an object whose only value is to confirm our suspicions or prove our point of view" (Rose 2016, 134 quoting Jackson 2013). Against this, he suggests that stories are in fact the origin of our thinking, rather than its evidence (see De Castro 2014). Thus, stories are not defined by what they prove, but "by what they give to us as authors" (Rose 2016, 132, see also Hendry 2007). This necessitates something of a reversal in how we conceptualise the relationship between "researcher" and "informant", and calls into question the scholarly preoccupation with originality, ownership and authorship. Rose points out that, in telling stories, "it is not us who give the [participants] a voice. It is they who give us ours" (Rose 2016, 144). From this perspective, as the "author" of this thesis I am not necessarily the voice that gives, but the voice that needs, and this necessitates a position not just of responsibility to research participants, but also of humility and vulnerability, for I rely on others and the stories they tell. As Rose explains:

When the stories that lead us to think are positioned as the evidence for our thoughts, we come to see language and writing as a tool that is ours to use and others as a proxy for its truth. It is only when we approach language as an opening to the other, only when we acknowledge the other's call as imminent to the very act of thinking itself, that we understand the thoughts we speak and the words with which we speak them do not originate from within, but constitute a response. (Rose 2016, 143)

Rose proposes that stories therefore have an innovative role to play in the academy: through stories, researchers can cultivate texts which are more inclusive and which acknowledge the author's debt to others; stories can open up academic disciplines to a multitude of voices; and they also open up a terrain of academic engagement where effectiveness is not only judged on its ability to match theory to empirics, but also by its ethics, its capacity to move and affect, and to contribute to social change. Thus, approaching the research from a perspective of story and narrative is appropriate given the aims of this research; as Bochner $(2012,162)$ urges, "there may be no better way [than stories] to come to terms with how we want to live and what we can understand and say about how others live than to listen to and converse with their stories". 


\subsubsection{Truth}

If, as I have suggested above, stories and narratives have an important place in academia, and in creating meaning, what can be said about the "truth" of such meaning? Here I share Bochner's (2012) approach in which he states that the truths he aspires to are not literal ones; they're emotional, dialogic, and collaborative truths. Therefore the stories we tell as authors are not intended to be received, but rather to be encountered, conversed with, and considered. He concludes that "my concern is not with better science but with better living and thus I am not so much aiming for some goal called 'Truth' as for an enlarged capacity to deal with life's challenges and contingencies" (Bochner 2012, 161). Collard et al. also evaluate the "value" or "truth" of particular stories according to their ability to enlarge one's capacity to live:

If different stories perform different yet interconnected worlds, then worlding practices can be evaluated in terms of their effects; some worldings might be wrong in the sense that 'they enact worlds (edifices) in which or with which we do not want to live, or that do not let us live or let some live and not others' (Collard et al. 2015, 328, quoting Blaser et al. 2014)

This stance fits with a pragmatist handling of the idea of "truth", that is, a "refusal to believe that truth 'rests upon foundations of any certainty"' (Harney et al. 2016, 319, quoting Bernstein 1992; see also Rorty 2009), and where the truth of an idea is judged by its usefulness in that particular context (Barnes 2008, 1544). Importantly, this approach is not an "anything goes philosophy" (as relativists might claim), but nor does it claim that truth can be "discovered" (as absolutists claim). Instead, truth is "constructed as the by-product of a process of solving problems undertaken by people in particular places and times" (Harney et al. 2016, 319).

As such, and in line with other attempts (noted in this chapter) to move away from neo-colonial forms of knowledge production, my approach adopts a pluralist view that there can be different kinds of truth which are context-specific. As Bauman (1997, 112) has pointed out, the idea of "truth" as referring to a singular reality belongs to a "rhetoric of power" which is founded on dispute that is, the concept of truth only becomes useful when it is used to assert the veracity of a belief in disputes about who is "right" and who is "wrong". This, as Bauman contends, is simultaneously a contest about who has the right to speak with an authority which others should obey, a contest which is most clearly evident in the logic of colonialism and imperialism 
and which has ongoing relevance for the ways in which academic/scientific knowledge is produced and circulated today (Noxolo 2017, Radcliffe 2017a).

However, Bochner (2012) asserts that the pluralism of a narrative approach does not mean it is not rigorous, or fails at "analysis" on the contrary, reflection is at the heart of storytelling because stories revolve around trouble, feelings, and decisions that need to be clarified and understood. For example, the evolution of my own version of this story has been constantly modified over the past three years, during which time the story has (I hope) been steered away from completely misleading interpretations be this through conversations with research participants, with supervisors, with friends, through reading and listening to other stories, and by presenting and writing for public audiences. Harney et al. $(2016,319)$ refer to this as a "critical community of inquirers" through which assumptions and beliefs can be filtered, examined, critiqued, and revised if necessary. Thus, stories rarely develop in a vacuum but they are shaped by an iterative process of inquiry which extends beyond my own subjectivity (Cronon 1992, Rose 2016).

With all that said, it also needs to be acknowledged that, through writing, life is turned into language, and yet, "the world does not exist in the shape of the sentences we write when we describe it" (Bochner 2012, 161, drawing on Rorty 1982). In writing, we can never fully "represent" (even if what I'm trying to do is represent my own response) the world because of what Dewsbury $(2003,1907)$ calls "the folded mix of our emotions, desires, and intuitions within the aura of places, the communication of things and spaces, and the spirit of events." Often, such traces "map out a world that we come to know without thinking" (ibid., emphasis added). Thus, perceiving, let alone expressing or conveying, such a folded mix of influences is rarely possible, although storying, as a more personalised, expressive and potentially affective method, as described above, may be better placed to do this than many other ways of "scripting" the world (Dewsbury 2003, 1907). In this thesis I have tried to combine "traditional" sources of knowledge (interviews, observation, textual analysis), with a simultaneous reflexivity about how my own "personal involvement with the world" (Dewsbury 2003, 1908) has influenced what I have, in the end, written. For this there can be no "proof"; no argument or discourse can necessarily make someone else feel it too, and yet it constitutes my knowledge, nonetheless. How to account for and recognise diverse kinds of knowing, from intuition ("gnosis") to external sources of information ("epistamai") is an ongoing endeavour in geography, as evident in the field of non-representational and more-than-representational theory (e.g. Thrift 2008, Lorimer 2008, Anderson 2009, Pile 2010). Such concerns resonate 
with Bataille's (2004) theory of "non-knowledge" and "inner experience" as aspects of life which fall outside the usual theories of "knowledge", but are nonetheless central to the experiences of life things like laughter, chatting, anxiety, and love, for example. Fitznor (2012) urges that the time is ripe for more personalised approaches to (non-)knowledge production, such as storying, narrative, and autobiographical writing, to be acknowledged as valid forms of academic scholarship and as an important contribution to countering neopositivist, neocolonial trends in social science. This will require a "shift in which bodies, which knowledges, and which ways of knowing are at stake in the academy" (Cameron et al. 2014, 24). Recently, such an approach has been taken up in "creative-critical" and "geopoetic" (Hawkins 2013, 63) modes of geographical writing (e.g. De Leeuw 2012, Cresswell 2014, Simpson 2015, Griffiths et al. 2017, Gerlach 2017b), which have also guided my own approach in this thesis.

\subsection{Who with, where, and why}

\subsubsection{Perspectives on place}

Initially, I have to admit, I felt frustrated as I watched colleagues and friends travel to exotic locations for their own geographical research, while I stayed in Wales. Wales seemed small, parochial, and in the broad scheme of socioecological challenges and grand ideas such as the "Anthropocene" insignificant. In retrospect I realise that my attitude was deeply rooted in a bias towards thinking of "the global" as glamorous, and the local as boring. Through my background in environmental sciences I had learned to think about the earth as a system and it felt like a wrench to be diverted from this "global" gaze in order to focus on the seemingly mundane circumstances of my immediate environment. The global appeared to me as "sufficient, whole, powerful, and transformative in relation to ... the local [which] is deficient, fragmented, weak, and acted upon" (Gibson-Graham 2002, 7). Indeed, in a seminal work on scale in geography, Taylor $(1982,26)$ emphasises the global as the "ultimate" scale, the one that "really matters". Gibson-Graham (2002) (amongst others) have passionately opposed such a framing, and yet notice that their own work on localised economic politics repeatedly stimulates these kind of reactions. Moreover, they contend that the perceived binary between global and local doesn't seem to emanate from a reasoned intellectual position, but a bodily, visceral one. They highlight an implicit assumption that "global" forces are inevitably more powerful (read, more 
interesting) than local interventions, and ask why it is that many researchers concerned with how the human and ecological consequences of globalisation might be transformed are not more often attracted to the local as a site of realistic challenge and possibility. Local stories, they claim, are made to seem "patently ridiculous" (Gibson-Graham 2002,6) as ammunition for challenging the power and dominance of the global, and yet, they claim, to partake in such an assumption is a mistake, for the local is just as much a space of politics as the global.

My own perspective has gradually changed over the past three years, and I have come to see how a focus on the local can not only be interesting, but it might be imperative with regards to socioecological transformation. Ingold (2000), drawing on Heidegger's (1971) concept of dwelling, has stressed this point, arguing that the conservation ethic entailed in a "world picture" representation of the earth (Skocz 2009, 130) not only has its roots in the history of colonialism, but it is also one which apparently expels humans from their immediate lifeworlds, and disempowers local communities of the responsibility to care for these environments because it shifts power/blame/agency "up there" and somewhere else (Friedman quoted in Marston et al. 2005, 427). As I see it now, this research is, in essence, concerned with how humans relate to and live within webs of more-than-human life, now and in the future, and ecology, as McIntosh (2012), suggests, "must start with the ground on which we stand". He adds that "[t]he parochial is that which relates to the parish, from the Greek, para-oikos, 'beside the household'. Ecology shares the same root in oikos. As ecologists, we must dig from where we stand, but that does not mean we should remain stuck in a hole" (Mclntosh 2012, 34; see also Padrutt 2009, 18). ${ }^{1}$

Perhaps this merely reiterates the popular slogan "think global, act local". However, such a notion suggests a binary position of "global" and "local" which, in actuality, is no more helpful for understanding the world, or developing a socioecological awareness, than a dominant global gaze is (e.g.Marston et al. 2005, Massey 2005). Indeed, "global" and "local" might be understood as rather arbitrary markers of scale, and to rank one scale above the other would be misleading (Marston et al. 2005). Instead, as Latour $(1993,122)$ states, local and global "offer points of view on networks that are by nature neither local or global, but are more or less long and more or less connected". In a similar vein, Bingham $(1996,365)$ writes that "the "material' and the 'social' are always already bound together, always already binding together". Thus,

\footnotetext{
${ }^{1}$ Harvey (1996, 169-172), however, tempers the attraction of place-based "bioregionalism" by pointing out that such ethics - while holding out some potential for addressing the current problems of human alienation from nature - also carry the risk of fostering overtly nationalist, exclusionary, even fascist ideologies.
} 
Massey describes how the local is global, for "places are also the moments through which the global is constituted, invented, coordinated, produced. They are 'agents' in globalization"(Massey 2004, 11, emphasis in original). From this perspective place is not a thing but a way of seeing and focusing an "entry point" into an issue or problem (Gibson-Graham 2002, 9). Thus, as Cloke et al. (2004) suggest, researchers should strive to see the intersections of influences emanating from different scales, rather than focussing on a place as though it where a bounded entity. This is, in general, how I have approached my engagement with the case studies in this thesis as particular "moments" which might be understood as constituted of, and constituting, broader processes of socioecological transformation.

However, as Cloke et al. (2004) also point out, in practice it is often difficult not to give priority to one "scale" over another during the course of fieldwork, and in my case the fieldwork foregrounded the perspectives of individual people and small communities and organisations in and around the region of South Wales where I live. On this point, it is interesting to note Marston et al.'s (2005, 422) contention that "most empirical work is lashed to a relatively small number of levels body, neighbourhood, urban, regional, national and global. Once these layers are presupposed, it is difficult not to think in terms of social relations and institutional arrangements that somehow fit their contours". Thus, form determines content, and against this they suggest to eliminate the concept of "scale" altogether, doing away with any transcendent predetermination "whether the local-to-global continuum in vertical thought or the origin-to-edge imaginary in horizontal thought" (Marston et al. 2005, 422). Against notions of scale, they too suggest an approach to space not through the "familiar concept of 'the local', but rather as the milieu or site actualized out of a complex number of connective, potential processes [...] [b]y this, we mean that a social site is not roped off, but rather that it inhabits a 'neighbourhood' of practices, events and orders that are folded variously into other unfolding sites". Inspired by Deleuze and Guattari's $(1987,500)$ call to "[n]ever believe that a smooth space will suffice to save us", Marsden et al., along with Massey, Gibson-Graham and many others, contend that what is needed is attention to the "fleshy, messy and indeterminate stuff of everyday life" (Katz 2001, 711), as the diverse and varied worlds of social life where "ideas are formed, actions are produced, and relationships are created and maintained" (Marston et al. 2005, 427). Place thus becomes a means through which to "tell new stories about the world", as Massey and Thrift (2003, 276) have written. Similarly, Bawaka Country et al. (2016, 469) suggest that the task is not to abandon the "big stories", but rather to look for the big stories which emerge 
from the richness of context, to look to "smallish stories" to build more nuanced understandings of place/space.

While such arguments over definitions of space and place might seem rather abstract and perhaps irrelevant in terms of my methodology, I think it is essential that I set out how my thinking and approach have been shaped by these arguments, and also why, therefore, I think a "parochial" perspective is justified and important. There is always a politics to scale, and how we engage with it has important repercussions for social action (2003). As a researcher, then, my engagement with scale informs my decisions regarding "how best to link social movements, for identifying cracks in perceived 'armours', and for highlighting social alternatives" (Marston et al. 2005, 426).

Carrying out research within the locality of where I live has had some additional, practical, benefits: it has been relatively easy to allow knowledge to grow "from the crucible of lives lived with others" (Ingold 2014, 387), more so than if my fieldwork had been restricted to brief visits abroad each summer (for example). In Bawaka Country et al.'s (2016) thinking on place, they contend:

no work purporting to be conceptual occurs without being firmly shaped by the empirical in place/space. The most philosophical of ramblings are shaped by the work of someone who is sitting (walking, dancing, eating, breathing, writing, reading), doing something, somewhere. [...] [I]t is not possible to write about knowing place/space without doing place/space (Bawaka Country et al. 2016, 470)

Even when I have not been officially carrying out fieldwork, I have been within a certain sphere of day-to-day life, events, news, emails, current affairs, social media updates, and conversations, which all have relevance for my research topic. While it has been impossible to formally record all of these influences as "data", they have nonetheless contributed to my sense of place, and of the themes and ideas arising. "Digging where I stand" (Mclntosh 2012, 34), then, has not only benefited my ability to learn from others and to understand their stories, it has also enhanced my own sense of place and belonging to this area, an area to which I myself was a newcomer five years ago. For me this has been a process of becoming more empowered about my own ability and responsibility to care for the ecologies (human and non-human, organic and inorganic) in which I dwell, and a shift in my perspective on the apparent hierarchy of local and global as powerless and powerful (respectively). Such a shift 
resonates with Gibson-Graham's $(2002,14)$ call for a "resubjectification", which they describe as a micropolitics of enabling subjects to inhabit terrains and identities differently, and reshaping the ways, as individuals, we live and enact the power of the global (see also Guattari [1989] 2014). Relatedly, Thrift (1999) suggests that it is through our embodied relationships and constant, concrete, attunements to the world that we make steps towards what he calls "an ecology of place."

However, with all of this said about the benefits of a place-based approach, one of the most formative experiences of my $\mathrm{PhD}$ (although not a part of my fieldwork) was a three month placement at Arizona State University (ASU) in the USA. ${ }^{2}$ The benefits to my knowledge, understanding and perspectives which came from this trip are hard to overstate, and this has prompted me to reflect further on issues of place/space and the role of mobility in research and in life more generally. Somewhat ironically, perhaps, being introduced to aspects of indigenous culture and knowledge in Arizona led to my interest in concepts of indigeneity, dwelling, and decolonialism in Wales. These concepts and literatures have helped me to see the research I have been carrying out in a different light, and which have subsequently strongly influenced the development of this thesis. That I travelled approximately 8000 kilometres to gain these perspectives only hints at some of the complexities of advocating a "placebased" approach, when the benefits of, and opportunities to, travel are also hard to ignore. In addition, the carbon emissions entailed in flying to the USA felt to me rather hypocritical in the context of a research project concerned with environmental futures. The prevalence of flying in academic disciplines such as geography which simultaneously claim concern for environment and climate change, is also an issue which is rarely discussed openly (some notable exceptions include Anderson 2013; 2014a), and again my trip to Arizona prompted me to reflect at length on this tension (although I have yet to reach any comfortable conclusions).

\subsubsection{Case study choice}

In the following sections I give an overview of each of the main case studies which became the foci of my research. Aside from the six mentioned here, there were several other organisations with whom I established relationships and even carried out interviews but which I have not included here, because, for one reason or another,

\footnotetext{
${ }^{2}$ This was made possible by the Economic and Social Research Council's Overseas Institutional Visit program. I participated in the Imagination and Climate Futures Initiative at ASU, and met with academics in this field at ASU and at Arizona University, Tucson.
} 
I did not engage with the group on the same level as I would have liked (either because of difficulty in contacting people and a lack of opportunities to attend events and meetings), or simply because I found that other case studies drew me in more interesting directions. There are almost endless possibilities for case studies when it comes to research on sustainability it seems that virtually everyone is "doing sustainability" in one way or another. Thus, inevitably the case studies presented here are just a "snapshot" of things going on in Wales. However, although they are in some ways "only" examples of themselves (Dewsbury et al. 2002, 439) they are also, as the previous section emphasised, the products and producers of multiple unfoldings and infoldings of social life, and as such one should be able to interpret from them something about wider socioecological contexts. While it should be kept in mind that the thesis would undoubtedly have taken a different shape, and different ideas developed, if I had chosen (or chanced upon) a different constellation of case studies, the fact that these particular case studies came to my attention and seemed to me to correspond to the research questions in particular and interesting ways indicates that they are not entirely random choices, either. I focussed on these examples because they all present in some way a novel, pioneering, or unusual approach to socioecological transformation at least within Wales, if not more widely. In addition, I was drawn to them because they cover a range of "sectors" governance, agriculture, energy and art and therefore present a wider and more diverse constellation of imaginaries than if I had focussed on one particular sector. I felt that breadth was more valuable than depth for the purposes of this thesis, given my intention of foregrounding ideas and practices which might otherwise be marginalised, as well as highlighting the textures, rather than the "smooth spaces", of life (Deleuze and Guattari 1987, 500). For each case study introduced below, I briefly describe what each does, why I was drawn to them, and what the nature of my involvement with each has been, including the kinds of methods used.

\subsubsection{Welsh Government}

The Welsh Government featured in the $\mathrm{PhD}$ proposal from the start, owing to its policy innovations on sustainability, environment and well-being (as described in the previous chapter). This political context in many ways sets the scene for this research, as I am both interested in the kinds of stories and narratives the Welsh Government is telling through this policy approach, as well as how non-governmental groups are imagining futures/responses in contrasting ways. 
As a result of the public nature of the development of the Well-being of Future Generations Act (WFGA) (including a series of public consultations called The Wales We Want), there have been many opportunities for me to attend events, briefings and conferences. I was doing this before starting the $\mathrm{PhD}$, out of personal interest, and have continued to do so throughout the $\mathrm{PhD}$. In most cases I took observational notes and recorded or noted down key speeches. However, although attending all of these events has helped to form my overall impressions, owing to time constraints I have only analysed a relatively small proportion of these notes using more formal methods such as coding.

In addition to observational material, I conducted two in-depth interviews with prominent figures related to the WFGA: Peter Davies, who was, until 2016, The Future Generations Commissioner, responsible for overseeing the development of the Act and the public consultations; and Andrew Charles, Head of Sustainable Development for the Welsh Government. I also interviewed Jane Davidson, the Government Minister who was responsible for creating the One Wales: One Planet Sustainable Development Scheme in 2009, which strengthened the Government's constitutional commitment to Sustainable Development as a central organising principle. Although Jane Davidson is no longer in Government, her influence is tangible and she remains a prominent and well-known figure in sustainability and environment issues in Wales more broadly.

Research has also involved textual analysis of Welsh Government material related to environment and sustainability, for example the One Wales: One Planet Sustainable Development Scheme and two short films which explain the WFGA Act and Environment (Wales) Act 2016. I have focussed on public-facing media as I was interested in the kinds of narratives and stories that the Welsh Government (and affiliates) convey to the public. Textual analysis has been one of the most useful methods because often it was difficult for me to engage on a more personal level with staff: government officials that I interviewed were often reasonably guarded due to the public nature of their roles. At most conferences and events I was also something of an "outsider", neither in government, business, nor public services. By contrast, the texts I have analysed are aimed at citizens like me and thus have provided a useful insight into the Welsh Government's values and narratives which are not necessarily obscured by problems of my positionality. 


\subsubsection{Ghost Forest environmental art installation}

The National Botanic Garden of Wales (NBGW) was also written into the PhD proposal as a partner, and my involvement with this organisation goes back to 2011, when I carried out research for my MSc dissertation there. The NBGW is Wales's only national botanic garden, and receives about 1.8 million visitors a year. As the first major garden to open in the UK in over 200 years, it has emerged with a strong stated commitment to educate and inspire for a more sustainable future, in line with the Welsh Government's own sustainability agenda (Welsh Government provides approximately $30 \%$ of funding for the Garden).

While my MSc project focussed on visitor engagement at the Garden as a whole, my $\mathrm{PhD}$ research has focussed specifically on the Ghost Forest, an environmental art installation created by the artist Angela Palmer, that arrived at the Garden in 2012. It comprises ten huge primary rainforest tree stumps which were sourced and transported from Ghana by Palmer and were subsequently displayed in London, Copenhagen and Oxford before coming to rest at the NBGW. Palmer's intention behind the work is to create a message about deforestation and climate change, with the missing trunks and canopies of the trees serving as a metaphor for the removal of the world's "lungs" a message which the Garden seeks to retain, albeit in subtly altered ways.

My interest in the Ghost Forest centred on three questions: one, what kinds of feelings and atmospheres around environmental futures does the Ghost Forest convey and, two, what kinds of responses does it evoke in those who encounter it? Thirdly, what does the Ghost Forest say about the role of art in socioecological transformation what is art deemed to "do", and with what effect? Essentially, I was interested in the kind of story the Ghost Forest tells about environmental futures, humans' relationship to the non-human world, and the role of art in our societies.

The research period with the Ghost Forest during the summer of 2015 was relatively constrained compared to other case studies, involving several days of observation and short, "vox-pop" style interviews with Ghost Forest visitors (eighteen people in total), whom I approached at random as they passed through the open air installation. In addition I carried out in-depth interviews with NGBW staff: Rosie Plummer (then Director), Bruce Langridge (Head of Interpretation), Rob Thomas (Head of Development), Simon Goodenough (Curator), and the Ghost Forest artist, Angela Palmer (via email). I also interviewed Lowri Jenkins, a member of staff at Size of 
Wales, a Cardiff-based charity who were instrumental in bringing the Ghost Forest to Wales. Textual analysis of Ghost Forest interpretive signs, a film about the making of the Ghost Forest, and website content (from the NBGW and the Ghost Forest) was also carried out. I also analysed comments left in visitor books from all three of the Ghost Forest's previous locations (Copenhagen, London, and Oxford), which helped to contextualise the comments I was receiving from visitors on site at the NBGW. I have also drawn on my own personal experience and encounters with the Ghost Forest, having spent several hours sitting amongst the trees and observing passers by.

\subsubsection{Swansea City Council}

Swansea City Council has been selected by the Welsh Government as the model local authority (LA) for sustainability in Wales (it has the largest team of people dedicated to sustainable development of all LAs in Wales, and receives additional funding from the Welsh Government in order to develop the Council's capacities in sustainable development. As such, the Council has been trialling a horizon-scanning exercise to produce periodic Future Trends reports for the LA. Although no other LAs are currently doing this, the Welsh Government has a constitutional commitment under the new WFGA for the Government to produce a similar Future Trends report twelve months before any Assembly election. I was therefore interested to learn how Swansea Council are interpreting the duties handed to them by Welsh Government, and also how the organisation imagines the future, particularly through the use of tools like horizon scanning.

I carried out in-depth interviews with three members of Swansea Council's Sustainable Development Unit, and an additional interview with a Learning Support Officer at Swansea City Council who works in some of the most deprived wards in Swansea, and has been involved with some public engagement activities related to the Future Trends report for Swansea.

In addition to interviews I attended a Swansea Council workshop in 2015 in which the Sustainable Development Unit introduced the concepts of scenario planning, foresighting, and horizon scanning to other members of Council staff. Textual analysis of Swansea City Council's Corporate Plan 2015 17, and of internal documents related to the Future Trends work, was also carried out. 


\subsubsection{Zero Carbon Britain}

Zero Carbon Britain (ZCB) is the flagship research project from the Centre for Alternative Technology (CAT) in Machynlleth, mid-Wales. CAT is a long-standing and well-known environmental charity in Wales, and ZCB was established in 2006 in order to research options for rapid decarbonisation in Britain. Since then, ZCB has produced three reports which demonstrate how Britain could achieve net-zero carbon emissions using currently available technology, efficiency improvements, and lifestyle changes. Importantly, however, ZCB stresses that its intention is not to provide a blueprint for action but rather to present an alternative vision of the future and to show what is possible. ZCB engages in public outreach about its work both on-site at CAT and further afield, through online resources, public talks, and residential courses. ZCB's vision has also been presented to the All Parliamentary Climate Change Group, was favourably received by the Liberal Democrats in 2007, and ZCB's roject co-ordinator, Paul Allen, sits on Wales's Climate Change Commission, an advisory board to the Welsh Government. ZCB has also presented its reports at several of the United Nations's Climate Change Conferences, including the most recent ones in Paris (2015) and Marrakesh (2016).

I was drawn to ZCB as a case study because it is setting out a bold, clear vision of the future and has an unashamedly utopian stance in fact, much of its work revolves around a belief that society needs more utopian visions of the future to aspire to. Although ZCB has a UK-wide focus, its links within Wales are also notable, and its work is referred to in the Welsh Government's Climate Change Strategy for Wales (2010).

My research with ZCB consisted of ten days of participant observation on site at CAT during August 2015, during which time I volunteered with the ZCB team (which, at that time consisted of three other people) and gave daily presentations about ZCB to CAT visitors. The presentation was one which had been designed by ZCB's coordinator Paul Allen, entitled "The Extraordinary Story of Humans and Energy", and gave an overview of how energy use has changed over the course of human evolution, the problematic situation this has created in regard to climate change, and finally an overview of ZCB's work and vision. During this period I also interviewed Paul Allen and Philip James (another ZCB team member), as well as CAT's director Adrian Ramsey and two members of staff at CAT who are involved with ZCB's work. I supplemented my volunteer experience with textual analysis of several online ZCB resources, including a short film made about the project. As with the NBGW, while 
on site at CAT I also carried out a number of opportunistic "vox-pop" interviews with visitors, speaking to fifty-two people in total.

\subsubsection{Emergence}

Emergence is a small, grassroots, arts and sustainability organisation based in Swansea, Wales, originally founded as a project by Fern Smith and Rhodri Thomas. It describes itself as "a collaborative project that advocates creative practice for a sustainable future through hosting artful events and gatherings". ${ }^{3}$ I was introduced to Emergence in the first year of my $\mathrm{PhD}$, when one of my supervisors forwarded me an invite to an Emergence event, a four day walk across the Gower Peninsula. Since then I have been more or less continuously involved with Emergence as both a participant and co-organiser of events. In 2015 I became a member of Emergence's small board of directors as it established itself as a Community Interest Company. Over this period I have developed a close friendship with Fern Smith and Philip Ralph, Emergence's codirectors, as well as several other people who are regularly involved with Emergence's activities. Consequently, my research with Emergence has been the most immersive of all the case studies I have been involved with, and Emergence has become a significant and influential part of my life in general. I have absorbed the Emergence "story" over many conversations and encounters, and the ideas and philosophies which have been introduced to me through it have been pivotal to how this research has evolved, and have challenged and transformed many of my own preconceptions about art and social change.

Participant observation with Emergence was based on nine separate events which I attended between 2014 and 2016, some of which I also helped to organise. In addition, conversations and presentations at some of these events were recorded and transcribed, and textual analysis of Emergence material online and in print has also contributed to the research.

Although Emergence is a small organisation, it is not unknown in Wales. It was behind the report Culture Shift: How artists are responding to Sustainability in Wales (2014), which gained some recognition in the Arts Council of Wales; Fern Smith, Emergence's founder and director, has been recognised within Wales for her leadership in the Arts with two awards from the Arts Council of Wales, one in 2009 and another

\footnotetext{
${ }^{3}$ Available at http://www.emergence-uk.org/\#the-emergence-vision. Last accessed 17 November 2017.
} 
in 2016. Meanwhile, the Welsh Government's Well-being of Future Generations Act (2015) includes amongst its seven well-being goals "A Wales of vibrant culture". Thus, Emergence's ideas even if currently only realised on a very small scale with a handful of people have a particular relevance in the Welsh context.

\subsubsection{Cae Tan}

Cae Tan is another initiative based near Swansea, in Ilston on the Gower Peninsula. It is the largest Community Supported Agriculture (CSA) scheme in Wales, and the only biodynamic one, providing weekly vegetable boxes for one hundred households across Gower and Swansea, and offering volunteering, training, and education opportunities for schools and youth groups. I first became involved with Can Tan as a member when it was established in 2014, and started to spend days on the Cae Tan field as a volunteer during the summer and autumn of 2015.

Cae Tan interested me as it has a particular vision and model of agriculture which claims to be more socially, economically, and ecologically sensitive. It is also part of a growing CSA movement currently still relatively small in the UK, but particularly popular in Japan (where it originated), and the USA. ${ }^{4}$ The biodynamic method that Cae Tan uses also presents some interesting and alternative ideas and practices about how humans can relate to the more-than-human world. Cae Tan has been featured on BBC Radio Wales, and is an active member of the CSA Network UK. It has run several successful crowd-funding campaigns to help support the farm, including regular community fundraising events such as harvest festivals, and has been in receipt of some Welsh Government funding during its first few years.

I carried out in-depth interviews with Cae Tan's main grower, Tom O'Kane, and another key figure, Ant Flanagan, who leases the land to Cae Tan and who initiated the project. I also interviewed two Cae Tan members who regularly volunteer at the field. In addition to participant observation during volunteer harvest days, I have attended public meetings about Cae Tan, participated in building a compost toilet on site at the farm, and joined in with one of the biodynamic preparations in 2015 (these take place on certain days each year and involve particular practices and rituals). As with Emergence, I have also developed friendships with many of Cae Tan's growers and members.

\footnotetext{
${ }^{4}$ See https://www.ifoam.bio/en/community-supported-agriculture-csa. Last accessed 17 November 2017.
} 


\subsection{Ways of working}

Having described my approach to the research and the case studies I have engaged with, my aim in this section is to provide an overview of the kinds of methods I've used, and why. The word "method" has associations with "normal" science and laboratory experiments, and can often be interpreted to mean techniques of "extracting" knowledge from the object of study. However, the etymology of method denotes a "way", "path, or "road" to knowledge (Doel 1994, 128), and this, as I hope I have already shown, more closely describes my own approach. In addition, Ingold (2014) describes participatory social science methods as, essentially, practices of joining in, and need not be shrouded in mystery. Nor should they, he claims, split experience between being in the world and knowing about it, for,

in the conduct of our research, we meet people. We talk with them, we ask them questions, we listen to their stories and we watch what they do. In so far as we are deemed competent and capable, we join in. There is nothing particularly special or unusual about this: it is, after all, what people do all the time when they encounter one another (Ingold 2014, 386)

Accordingly, I have not designed the research, fieldwork and analysis in particularly "neat" or linear ways, but rather have sought to immerse myself in various encounters, while keeping my research questions in mind. For example, I initially approached a multitude of organisations and people as potential participants in the research in some cases I carried out interviews with them, too. Inevitably, while some of these encounters opened up avenues for research, others didn't, while still others would morph into something I hadn't expected but which was equally interesting. To have set out with a very rigid sampling strategy, interview design, or "tick-list" of people and organisations, would have prevented me from being able to follow unforeseen lines of enquiry. Similarly, I resisted defining particular "phases" of the research, so as to afford me similar flexibility to be open to unforeseen circumstances and opportunities that arose all the time. I carried out participant observation, interviews, and textual analysis (as discussed later in the chapter), more or less concurrently throughout my fieldwork-intensive second year. One determinant of timing was, however, that I usually waited to ask for interviews until I felt I had built up a sufficiently good relationship with that particular person, and had a sufficiently good understanding of their work. My use of several different methods (interviews, participant observation, 
and textual analysis), can also be understood as my attempt to "join in" in various ways, rather than a desire to produce a "complete" picture by "triangulation" which would be, as Hammersley and Atkinson note (1995, 230), a naive ambition. Using a variety of methods has, however, enabled to me to understand future imaginaries from a variety of perspectives, and has also afforded me some flexibility with regard to the degree I was or wasn't able to "participate". For example, in some cases my primary mode of engagement was through participant observation and interviews, whereas others where opportunities for more direct involvement were limited relied more upon documentary sources.

While this might indeed sound like "mess in social science research" (Law 2004, 2), that is, in part, the point. Davies and Dwyer (2007) urge researchers to rethink exactly what it is that they understand social science investigations to do, and how methods are being used to make claims about the world. In particular, they suggest that the operation of social science methods to generate singularity, clarity and to reduce uncertainty and ambiguity is misleading. Rather than the pursuit of certainty in generating representations of the world, Davies and Dwyer suggest that there is a need to recognise "that the world is so textured as to exceed our capacity to understand it, and thus to accede that social science methodologies and forms of knowing will be characterised as much by openness, reflexivity and recursivity as by categorisation, conclusion and closure." (Davies and Dwyer 2007, 258). Thus, and like Haraway's (1994) call for researchers to participate in making worlds, Law (2004) and Davies and Dwyer (2007) contend that the methods that researchers use don't just describe social realities but help to create them, too.

This has implications, ultimately, for how I conceptualise what it is I "do" as a researcher. Initially, I thought what I was doing was "ethnography", an attempt to accurately note down and record what it was I was encountering literally, to write about people. For Ingold, though, in taking such an approach

the priority shifts from engagement to reportage, from correspondence to description, from the co-imagining of possible futures to the characterization of what is already past. It is, as it were, to look through the wrong end of the telescope. Instead of calling on the vision afforded by our education to illuminate and enlarge upon the world, the ethnographer takes his or her sightings from the world in holding up the other's ways to scrutiny (Ingold 2014, 392). 
Embracing the "mess" of social science, admittedly, can create difficulties when it comes to analysis and writing. But the benefits of a messy approach are that it has freed me to participate in the "co-imagining of possible futures" (Ingold 2014, 392), rather than simply reportage and description aspirations which, as I stated in the opening section, have been central to my research aims.

The problem, as Greenspan (1998) has observed, is not what we get out of stories but how we can get into them. In attempting to "get into" stories about socioecological transformation in Wales, I find the concept of a "meshwork" (Ramsden 2017, 4) useful, for it describes how theory and practice intertwine in producing knowledge. A "meshwork" is also an appropriate concept because, as I progressed through the $\mathrm{PhD}$, I found the confidence to be less rigid about where I drew the lines around what my "methods" were and what "the field" was. As already intimated, "research" has not necessarily been something I stopped doing (knowingly or unknowingly) when I left the office or turned off the voice recorder. With this in mind, I now turn to describe in more detail the specific methods which formed the core of my approach, and the basis of my "meshwork".

\subsubsection{Conversation as research method (a.k.a In-depth inter- views)}

I interviewed thirty-five people using a semi-structured, in-depth approach. I approached potential interviewees on the basis of their involvement with the various case studies: usually at least one of the founders/directors of the organisation, as well as additional interviews with key staff, volunteers, or participants. I conducted a few interviews with people connected to case studies which I subsequently decided not to include in the thesis. However, these people's responses to the more general questions (below) have contributed in a broader sense to my understanding of the research topic. Each interview was tailored towards finding out about the socioecological imaginaries of the case study and of the people involved with it, and so the form was often very unstructured, allowing the interviewee to initiate topics which I could not have anticipated. However, towards the end of every interview, I steered the conversation towards the same set of three questions, which (although sometimes phrased in slightly different ways) were:

- How do you imagine the future? [I left this question open regarding temporal and geographical scales] 
- What's one thing that excites you and one thing that worries you about the future?

- If you put yourself in the shoes of someone living in the future, looking back at today's society, what will they think?

These questions were deliberately abstract and sometimes took people by surprise, but they were often very useful for eliciting people's "knee-jerk" responses to, and imaginations of, socioecological futures. In my analysis of interview material, these questions have been helpful both in serving to contextualise people's involvement with a particular case study, and also as a more general insight (irrespective of case study) into how people feel about and imagine socioecological transformations and the future.

The location for interviews was always suggested by the interviewee (two interviews were conducted by phone), and ranged from cafes and pubs, to people's homes and offices, and even a polytunnel. I did not set any parameters as to the length of each interview (they ranged in length from thirty minutes to nearly two hours). In all cases my intention was to create a relaxed and friendly atmosphere in which conversation could flow and we both felt at ease. Nonetheless, interviewing people was by far the most nerve-racking and difficult aspect of the research for me: due either to the awkwardness of speaking to people I otherwise knew little about, or the opposite problem which was having to conduct "formalities" with people who I knew well and count as friends. Usually, in all but the most formal of situations (for example with government representatives), this awkwardness would dissipate and interviews often evolved into a more relaxed conversation. In some instances I got the impression that, in having the conversation, both I and the participant felt empowered through the sharing of stories, and talking about our concerns for the future. In this sense the effect of conversations went beyond simply research and became a form of participation in the community and world (Harney et al. 2016). Often, I was amazed at the depth of feeling that people expressed, and was humbled by the trust they showed in voicing such feelings (even in the short space of "vox-pop" interviews). As such, it feels more appropriate to describe many of these encounters as conversations rather than interviews. As Ingold (2014) contends, research sometimes need not be any more complicated than meeting people, and talking with them. Similarly, the word "interview" implies a particular subjectivity of the researcher as "in control", and this neglects the possibility that, as already mentioned, it is the interviewee who gives us our voice, not the other way round (Rose 2016). 
Uprichard has made the case for using semi-structured interviews to explore temporal horizons, arguing that "asking individuals about their desired and projected futures ought to be part of the social scientist's methodological tool box" (Uprichard $2011,4)$, because time and temporality are real ontological entities which shape the world, and are shaped by social life, too. Thus interviews are a way of constructing multiple possible futures through personal narratives, and of recognising the agency of individuals as "accomplices of the processes that help make the probable a reality" (Uprichard 2011, 12). Semi-structured interviews are also, I believe, suitable for capturing some of the complexity that time and temporality introduce to understandings of how socioecological transformation is imagined. Semi-structured interviews allowed the interviewees freedom to roam onto topics which I could never have anticipated, such is the potential diversity of people's imaginations. As Uprichard notes "the conversation can go as deep or as broad as may be necessary" (Uprichard 2011, 17). In addition, face-to-face interviews facilitated a sensitivity to feelings about the future that were conveyed through facial expressions, pauses, and laughter, for example (Clifford et al. 2010) all aspects which would not have been possible to acknowledge through methods such as paper questionnaires or online surveys.

Yet, despite the advantages that interviewing affords, Uprichard (2011) notes that in the social sciences, it is yet to be widely engaged with as a method for finding out about how people perceive the future. This is odd, she contends, given the popularity of interviews for finding out about the past and the present. Given the assumption that people can talk about the past and the present "it is rather curious that when it comes to asking individuals about the future, there might be a perception that individuals are somehow unable to talk about the future. This perception is simply unfounded" (Uprichard 2011, 17). In much social science research on future imaginaries, Uprichard suggests, people are rarely "allowed" to simply speak, or the researcher to simply listen (although, the notion of "simply" could be contested here, as there is always a context for conversations). For example, some common methodological approaches to studying future imaginaries include: web-based surveys to investigate how individuals think about and visualize the future (Tonn et al. 2006); longitudinal studies of large datasets in order to relate attitudes about the future amongst young people to outcomes in adult life (Alm 2011); psychological studies to investigate the relationship between the degree of optimism in individuals' outlook for the future, and the time horizon they extend their thoughts to (Schmidt et al. 1978); comparative studies (for example between groups of males and females) about how much people worry about the future; scenario-development or visioning workshops (see Arnaldi 
2008); analyses of representations of the future in a large corpus of English-language blogs related to climate change (Fløttum et al. 2014); a substantial body of work on young people's views of the future, conducted through nation-wide or transnational surveys and questionnaires (See Hicks 1996); a combination of quantitative surveys and qualitative discussion groups to elicit views of future social-economic and climate change in England and Italy (Lorenzoni and Hulme 2009), and photo-elicitation techniques for researching energy biographies (Shirani et al. 2016). In such research, there seems to be a preference to use what Bussey $(2014,95)$ calls rather "complicated and abstract" methods that have a tendency to create distance between researcher and participant.

Like Uprichard (2011), I am prompted to wonder why it is that semi-structured interviews, or, for that matter, conversations are often overlooked as methods in futures research. My own experience has been that the face-to-face communication and conversation-like rapport of semi-structured interviews actually afforded some of the most revealing encounters with other people's stories. With that said, and as mentioned in the previous chapter, questions about "planetary" futures did, understandably, sometimes take people aback and leave them struggling for words. In response to this, I organised a one-off experimental focus group/workshop as part of the Small Is festival held at CAT in September 2015. ${ }^{5}$ The workshop design was such that people could come and go as they pleased over the course of an hour and a half and chat about the future, while playing with some playdough which I had set out on the table. I was inspired by Gauntlett's (2013) premise that "making is connecting", and that, through making things, or even just having something to play with in their hands, people might relax and be able to express visually thoughts and feelings which they may never have been asked to put into words before. Although I only conducted one such workshop, it was a fascinating experience and shed light on some of the limitations of conducting one-to-one, and/or short "vox-pop" interviews, especially with regard to "big" topics (such as the future of the planet) which sometimes elicited bemusement, surprise, awkwardness and embarrassment from participants. In a group setting, I found that the participants would prompt each other with questions, and that conversation on these "big" topics flowed more easily.

There is one major assumption which underpinned my approach to the interviews and choice of questions which I feel needs particular attention here. This has to do with my own implicit conceptualisations of time and temporality as linear (an assumption which I only became aware of myself later in the research). In asking

\footnotetext{
${ }^{5}$ Available at https://www.smallisfestival.org/. Last accessed 17 November 2017
} 
people how they imagined the future "would be", or what people in the future might think "looking back" at today, I was conveying a particular view of the future as somewhere "out there", ahead of us. While it is likely that the people I interviewed, coming from a broadly similar cultural (that is, Western) context to me, held similar conceptions of time, it is possible that this was not always the case. For example, in one interview the person I was speaking to insisted that he struggled to think about the future, that he doesn't do it very often, and so couldn't answer my question at all. On reflection, I realised that my questioning had not allowed for the fact that, for many people and cultures, time is not conceived of as linear. For example, many Buddhist philosophies understand the notion of time flowing from past, present, to the future to be an illusion, and that obsessing over "the future" might in fact be detrimental (see Inayatullah 1993).

Ruby's (1991) paper about the dilemmas of "speaking for, speaking with, and speaking alongside" opens with an extract of dialogue from the film The Passenger (1975) to highlight how, in asking questions, indeed in having conversations, we are sometimes blinkered by our own particular perspective, which may have very little in common with the perspective of the person we are talking to. I reproduce the extract here because it reminds me as with the assumption about linear time that the conversations I have had with people during this research probably say as much about me as they do about other people, and it makes me smile to think that my participants may have shared the same bemused response of the rebel character (below), but were too polite to say! In the film, the character Locke tries to interview, on film, a rebel leader in an unspecified north African country. They have the following conversation:

Locke: Yesterday when we filmed you at the village, I understood that you were brought up to be a witch doctor. Isn't that unusual for someone like you to have spent several years in France and Yugoslavia? Has that changed your attitude towards certain tribal customs? Don't they strike you as false now and wrong perhaps for the tribe?

Rebel: Mr. Locke. There are perfectly satisfactory answers to all your questions. But I don't think you understand how little you can learn from them. Your questions are much more revealing about yourself than my answer would be about me.

Locke: I meant them quite sincerely.

Rebel: Mr. Locke. We can have a conversation, but only if it is not just 
what you think is sincere but also what I believe to be honest.

Locke: Yes, of course, but...

(The rebel leader now turns the camera around so that Locke is centred in the frame.)

Rebel: Now, we can have an interview. You can ask me the same questions as before.

(Ruby 1991, 50)

\subsubsection{Vox-pops}

In addition to in-depth interviews, I also carried out short "vox-pop"-style interviews involving about seventy people (interviews were often with two or three people at a time). All the vox-pop interviews were carried out at either the National Botanic Garden of Wales (NBGW), or at the Centre for Alternative Technology (CAT), as these were locations which provided ample opportunity to approach passers-by during my time spent carrying out fieldwork at each site. Each conversation would typically last a few minutes, and my intention was to get an insight into people's feelings and thoughts about the topics I introduced, rather than the kind of depth of knowledge associate with in-depth interviews. At the NBGW I asked people about their impressions of the Ghost Forest art installation (GF) and about their hopes/fears about deforestation, and at CAT I asked people about the purpose of their visit and about their impressions of the Zero Carbon Britain project. In each interview, as with the in-depth interviews, I also asked a final question(s) about how people feel about the future, either by asking what they were excited and worried about, or what they imagine the future will be like. The structure of this last question often varied according to the context and what I felt was appropriate.

The vox-pops were largely opportunistic and I didn't have a particular sampling strategy. My intention was simply to have short conversations with people around me, and if possible to find out something of their thoughts about the future. All of the participants were visitors to environmentally-minded, educational charities (NBGW and CAT), and so the range of people I was able to speak to was unavoidably biased. However, it is worth mentioning that the two organisations (very broadly speaking) attract quite different visitors, with CAT being a renowned "left-leaning" organi- 
sation, popular with those seeking "alternative" lifestyles, while NBGW might be seen as a more "traditional", "conservative" (with a small "c") organisation, popular with day-trippers, garden enthusiasts, and increasingly young families. In addition, both NBGW and CAT also attract visitors who are not necessarily interested in the organisation's environmental ethos and are simply passers-by. Finally, almost all of the people I spoke to were white and British and the majority were over the age of thirty reflecting the demographics of visitors to both organisations. ${ }^{6}$ Although these vox-pops should not be construed as in any way representative of a particular population, they did highlight some interesting themes which have helped to corroborate findings from the in-depth interviews, as well as raising some interesting questions regarding the mood of "non-expert" citizens in contrast to the kinds of narratives being produced by the "change-maker" case studies I explore. My intention was never for the vox-pops to constitute a central part of the research, but rather to complement it as an interesting sideline. Although few direct quotes from the vox-pops are used in this thesis, largely because the research focus moved towards in interest in the imaginaries constructed by the projects and organisations themselves, I mention them here because these conversations were an important aspect of my experience of the research process. They would also be a rich starting point for future research on individuals' imaginaries of the future.

\subsubsection{Participant observation}

Participant observation has been central to the research presented in this thesis. Ingold $(2014,389)$ describes participant observation as being a practice of attending "to what others are doing or saying and to what is going on around and about; to follow along where others go and to do their bidding, whatever this might entail and wherever it might take you". Similarly, Hammersley and Atkinson (2007, 3) state that this method involves participating "in people's daily lives for an extended period of time, watching what happens, listening to what is said, asking questions; in fact collecting whatever data are available to throw light on the issues with which [the research] is concerned". Thus, there can be no observation without participation to observe is not to objectify but to attend to persons and things, to learn from them. In essence, Ingold contends that participant observation is an (albeit condensed) expression of the way we go about our lives. The difference, perhaps, is that I would

\footnotetext{
${ }^{6}$ These are my own estimations - I did not ask people directly to provide demographic information as I wanted to keep the conversations as informal and relaxed as possible. I did not carry a note book or clipboard, just a voice recorder.
} 
often carry a notebook around with me, making note of things that I might not ordinarily have been as alert to. I did not try to disguise when I made note of things, although, equally, I tried not to be so overt as to make people feel uncomfortable. When it wasn't possible to make notes immediately (for example while walking, or weeding a vegetable patch, or having a conversation) I would take "scratch notes" (Ottenberg in Sanjek 1990, 92 121) and then write these up in the evening so that the experience was as fresh in my memory as possible. Thus, when spending time with these various groups of people and organisations, I was open about what I was doing and why I was there, and asked permission of the relevant people. I was careful to point out that my intention wasn't simply "research" but was also a matter of personal interest and commitment for me (as such, my involvement was generally one of a "complete participant", attempting to participate fully in the group's activities, as opposed to a more partial or detached role (see Ritchie et al. 2013, 246), although this varied according to the case study, as described above). In addition to notes, I also took photos as a means to remember and "capture" particular moments or themes. As Ritchie et al. (2013) advise, photos are also texts and should be subjected to the same reflexive thought as other observational data (namely, "why did I take it?"). Taken together, the insights arising from participant observation and my own photos, while crucial in helping me to develop my understanding, are distinct from material arising from interviews because observational texts are purely researcher-generated, and rely upon the assumption that my notes, memory, and diligence are sufficient (Mack et al. 2005). Nonetheless, in most instances I have spent sufficient amounts of time with the case studies that my impression of what they do has not been solely dependent on notes or photos made in the field, for some of what I "know" about each case study has built up intuitively, through those affective "traces" that Dewsbury (2003) refers to, though which I might not have directly observed or recorded in the field.

Participant observation thus provided a rich, immersive method of building an understanding of the narratives and stories of the case studies I was involved with, and it afforded an in-depth knowledge of the workings and ideas of particular groups often spanning several months or years. As Ritchie et al. (2013) contend, observation has the potential to facilitate insights into interactions, processes and behaviors that often goes beyond the understanding conveyed in verbal accounts. In addition, participant observation was crucial in getting to know and feel something of the "affective atmospheres" (Anderson 2009, 77) and non-representational aspects such as emotion, belief, and intuition, which were central to how the case studies functioned and the 
meanings they created. Participant observation has been used to great effect in geography in this regard, either as a means to gather "insider" knowledge of places and peoples the researcher is already familiar with, or as a means for becoming acquainted with hitherto unknown situations (Laurier 2016), so as to understand the world in new ways and from different perspectives ${ }^{7}$ (e.g. Parr 1998, Wylie 2005, Venkatesh 2009, Vannini 2012). Indeed, the researcher's experience, response, and physical and emotional feelings evoked by the contexts they are encountering is all part of where the value of participant observation is considered to be (Ritchie et al. 2013). Participant observation was also central to my ability to form relationships of trust with people who I subsequently interviewed, and owing to my regular participation with certain groups meant that my presence as a researcher, over time, did not "stand out" and cause people to behave differently from how they might otherwise have done. Finally, participant observation like in-depth interviews seems to be a relatively under-utilised method for engaging with the topic of socioecological futures. This is perhaps because, generally, participant observation is an excellent method for understanding the "here-and-now" and how the fabric of everyday life is produced, and thus notions of imagination and the future might not seem so readily available as things to "observe" (and participate in) in the material practices of the present. On the contrary, I found that, as long as I approached participant observation with a question in mind about what kinds of socioecological imaginaries are being produced, and what kinds of futures implied, then this method provided valuable insights into the research topic.

\subsubsection{Document analysis}

Doel $(2010,488)$ maintains that a "text" is a tissue of signs "it is anything with a signifying structure, a text is anything that refers meaning and thereby the reader elsewhere, to other texts, languages, codes, contexts, situations, expectations, habits, etc." As such, texts often appear as "mundane and are scattered among the debris of everyday life (e.g. graffiti, statutes, advertisements photographs)" (ibid. 485). In short, anything that means anything or can be interpreted is a text, regardless of whether it is a written source, and so to some extent textual analysis, as a method, applies broadly across all the strands of my research. However, I have engaged specifically with some written texts in order to analyse content from specific organisations,

\footnotetext{
${ }^{7}$ In fact, in some instances, my position shifted during the course of the research from that of an "outsider" seeking alternative perspectives, to an "insider", reflecting on my own (by then) close involvement with the case studies.
} 
particularly those which serve as "communicative devices" (Ritchie et al. 2013, 342), presenting the ideas and values of the organisation to the wider public. In analysing texts, I have found Doel's $(2010,491)$ style of approach useful. He suggests that of any text the researcher asks

- How, why and for whom has it been constructed?

- What are the materials, practices and power relationships that are assumed by it and sustained through it?

- What codes, values, dispositions, habits, stereotypes and associations does it draw upon?

- What kind of personal and group identities does it promote? And how do they relate to other identities? What does it mean?

- What are its main structuring devices: oppositions, divisions, metaphors, illustrations, examplars etc?

- More importantly, what kind of work does it do, and who benefits? What has been included, excluded, empowered and repressed? How might it be modified, transformed or deconstructed? How could this social space be inhabited differently?

- Finally, since nothing ever comes alone, what wider assemblages does it fit into and resonate with? Are these assemblages synergistic or contradictory?

Cultural texts matter because they shape and inform social practices and social spaces (e.g. Clarke 1997). As Atkinson and Coffey (1997, 55) state, "documents do not stand alone", they are always situated within a wider cultural context and frame of reference; they are "social facts which are produced, shared, and used in socially organised ways (ibid., 47). In many instances, documents were my first port-of-call in the research process, helping to direct me towards particular themes or subjects which I could follow up in interviews (e.g. Bowen 2009). Documents also provide a useful angle on certain themes and stories which can be less obvious when approached through interviews or participant observation. Often, documentary and website data (including images, graphics and photos) can provide the clearest insight as to a particular person's or organisation's vision, and what kind of "public face" they seek to present. Alternatively, what is and is not presented in documents can also be a useful and potentially insightful point of comparison with other accounts, 
such as those from interviews and participant observation. Content analysis is also, arguably, the least subjective of the methods I have used because the texts are not researcher-generated, (although, of course, my interpretation of them is). As these texts exist in the public domain, people can read them for themselves in order to, if they wish, verify or dispute my interpretation.

I have focussed on key, public-facing texts produced by each of the case studies most frequently website content but also government reports and communications, including short videos made for that purpose. Although some physical documents were obtained, very often the documents I analysed were downloaded from the internet, or taken directly from a web page. This in itself can raise some challenges for the researcher because of the transient nature of much internet content (Flick 2009). However, my interest was usually in published/official documents, or in the mission statements of organisations, which are generally more stable in this regard. One exception has been the Ghost Forest's official website, which was taken down in 2017. However, I managed to find other sources of the same material where necessary, and these sources are cited wherever they are used in the thesis. Finally, in addition to Doel's questions above, I have approached analysis of these texts always with my overarching research questions in mind, namely, what kinds of socioecological transformations are being imagined?

\subsubsection{Analysis}

As the notions of "meshwork" (Ramsden 2017, 4) and "mess in social science" (Law 2004 , 1) imply, analysis has not been, in this case, a discreet phase of the research which happens after fieldwork, before writing up. In fact, the analytic journey has been ongoing, and began long before the fieldwork itself did, from my choice of research questions (which defined the scope of the formal analysis to come), to hunches and opportunities which arose and shifted my attention to other aspects of the research (Ritchie et al. 2013).

Nonetheless, the "output" of the methods I have described above produced a lot of material; transcripts, field notes, and documentary texts, which I have needed to approach in a systematic manner in order to make sense of it. My basic approach has been that of thematic analysis, working systematically through texts in order to identify clusters of meaning, topics, and themes which help me to address the overall research questions (e.g. Boyatzis 1998). Thus, this approach had been inseparable 
from a grounded theory approach, allowing for theory to develop out of the themes identified, as the material accumulates, rather than analysing the material according to a particular theoretical framework (Glaser and Strauss 1967, Gibbs 2007).

Coding has been a useful way to get a handle on themes, although I have been wary of the way in which coding, in effect, is "an attempt to fix meaning, constructing a particular vision of the world that excludes other possible view points" (Seale 1999, 154), as this undermines my intention, as stated earlier, to take a pragmatist approach which doesn't seek to fix meaning but rather is more open to the world as in constant flux, with multiple "truths". For this reason, I have used codes "as signposts to interesting bits of data, rather than representing some final argument about meaning" (Seale 1999, 154, see also Cope 2005). In this respect, coding has been hugely beneficial in helping me to organise and sift through the material. I mainly coded using open-source coding software RQDA ${ }^{8}$ (a linux version of N-Vivo), although some coding was carried out by hand where the document formats were not compatible with the RQDA software (physical paper copies, or PDFs, for example). I approached the material non-cross-sectionally (developing separate code "books" for each case study (see Cope 2005) and cross-sectionally (applying the same codes to the material, regardless of the case study)(Ritchie et al. 2013). The later approach was used predominantly for analysing material from in-depth interviews and vox-pops, where I was interested in people's responses to the more general questions about the future, regardless of which case study they were affiliated with. The codes that I developed included a combination of descriptive and analytic codes (Cope 2005, 282 283 ), that is, some referred to blatant or obvious themes as described by the words and phrases themselves, while other codes referred to more implicit themes indicated by the text. I typically used these codes alongside one another as seemed appropriate as I worked through the text, rather than first coding descriptively and then going back to code analytically. Alongside these coding activities, I also kept a diary of ideas and themes which occurred to me along the way, both specifically in relation to each case study and more generally, particularly as I began to identify linkages or differences between case studies. The diary also helped me to keep track of insights and significant themes as they became intuitively clear to me, and thus the coding provided a useful means with which to revisit the data and corroborate (or not) my intuitive sense of things.

\footnotetext{
${ }^{8}$ Available at http://rqda.r-forge.r-project.org/. Last accessed 1 August 2017
} 


\subsection{Introducing the empirical chapters}

In this chapter my intention has been to give the reader an insight into how I have approached the research and why I have arranged the thesis in a particular way, stressing that what follows is a particular way of storying the experiences, encounters, and observations I have had during the past three years. Although I have not made explicit reference to aspects of assemblage theory in this chapter, Anderson and McFarlane's $(2011,126)$ description of assemblage as "a certain ethos of engagement with the world" encapsulates how I have gone about "assembling" this thesis, and how I hope the writing conveys a sense of this ethos. As Anderson and McFarlane (ibid.) suggest, "rather than the testing of a pre-existing hypothesis, work that deploys assemblage experiments in the sense that it opens the researcher up to risk, embraces uncertainty, expresses something of the fragility of composition, and strives to listen to what Deleuze and Guattari $(1986,83)$ term 'the sound of a contagious future, the murmur (rumeur) of new assemblages of desire, of machines, and of statements, that insert themselves into the old assemblages and break with them".

As outlined in the previous chapter, the following empirical chapters are structured according to themes which developed during the course of the research. These themes were not predetermined but emerged at a later stage in the research. In large part, each chapter theme became apparent to me because of some striking contrasts between two or more of the case studies, in terms of how the case studies dealt with a particular dimension of socioecological change. For example, through my research with the Ghost Forest and Emergence, I was struck by how differently each project conceptualised and performed the role of art in socioecological transformation. As such, although the themes of each chapter could be used as a lens on all of the case studies, I focus only on two or three main case studies per chapter those cases where the theme is particularly pronounced, or where a comparison between particular cases is helpful in highlighting some nuances and/or contradictions (occasional text boxes provide short contributions from other case studies where necessary). In addition, I settled on these themes because each of them, it seems to me, constitutes a fundamental aspect of socioecological imaginaries (that is, conceptions of time and change, relationships between humans and environment, and the role of art and culture), and the chapters therefore contribute to wider ongoing conversations about future imaginaries and socioecological transformation. The case studies appear in each empirical chapter as follows: 
- Chapter 3 ("Constructing time to build the future"): Welsh Government, Swansea City Council, Emergence, Zero Carbon Britain

- Chapter 4 ("Cultures of nature: imagining and performing more-than-human worlds"): Welsh Government, Cae Tan

- Chapter 5 ("The role of art in socioecological transformation"): Emergence, Ghost Forest (National Botanic Garden of Wales)

Finally, chapter 6 ("Reflections") summarises the thesis's key contributions, draws out some cross-cutting themes between the chapters, and indicates some possibilities for future research. 


\section{Chapter 3}

\section{Constructing time to build the future}

Time is one of the assumed yet irreducible terms of all discourse, knowledge, and social practice. Yet it is rarely analyzed or self-consciously discussed in its own terms. It tends to function as a silent accompaniment, a shadowy implication underlying, contextualizing, and eventually undoing all knowledges and practices without being their explicit object of analysis or speculation. (Grosz 1999, 1)

The original task of a genuine revolution ... is never merely to "change the world", but also and first of all to "change time". (Agamben 1993, 91)

This chapter explores how temporality and futurity feature in several of the case studies' orientations to, and narratives about, socioecological transformation. Time and futurity are often taken-for-granted aspects of climate change and sustainability, and thus tend to be under-examined, as the opening epigraphs suggest. I start from an understanding of time not as universal or independent of social life, but as socially constructed and as taking on shape by virtue of relational configurations between past, present and future (Klinke 2013, Anderson and Adey 2012). This does not mean to say that temporality doesn't have "real" influence on people's lives indeed it does both in material practices and in how people think (Adam 2013) but is rather to acknowledge that time, like the future, is made present in a multitude of implicit and explicit ways language, statements, practices, measures, 
apprehensions and engagements which influence how life is imagined and governed. Inayatullah (1990), following this Foucauldian perspective on time, describes how time is no longer a given: it is a way of creating rather than describing the world. Anderson and Adey $(2012,1533)$ write that "futures are folded into a dynamic present and ... all geographies are made through this folding and unfolding". If, as this suggests, constructs of time are complicit with organisations of power, knowledge, and institutions, then time and futurity are important considerations in discourses of environmental transformation, and in understanding how geographies (including relationships between humans and environment) are made and unmade.

My aim in this chapter is therefore to illuminate some strands of temporality and futurity which run through the case studies I have been involved with, so that they may be rendered more available for conscious consideration. I focus on questions of how transformation, and relationships between past, present and future, are imagined, asking what shape does time take in these imaginaries? And how is change assumed to occur? My interest, therefore, is not only in what these organisations are saying about the future but how they are saying it. In doing so, I use literature about philosophies of time, anticipatory politics, and complexity theory as a lens through which to consider contemporary empirical examples from Wales, and therefore help to further understanding of how time and futurity function in social and political life, and, ultimately, in socioecological transformation.

The chapter focuses on three main case studies: the Welsh Government, Zero Carbon Britain, and Emergence. Some additional material comes from my research with Swansea City Council. I begin by "setting the scene", giving a brief overview of the kinds of socioecological transformations these three organisations aspire to achieve or assist, before turning my attention to aspects of time and futurity which shape their approaches in various ways. Part one focusses on questions of uncertainty and how it is handled; part two explores the kinds of trajectories of change imagined by the case studies; and part three explores how the various case studies set out to "know" the future if at all. Part four concludes with some reflections on the inherent tensions and contradictions that a focus on temporality and the future brings to light. Not every section features all three case studies, but instead I bring different case studies in and out of focus depending on the themes under consideration. 


\subsection{Transformative visions}

I was drawn to each of the case studies in this research because of the claims they make and aspirations they have with regard to future socioecological transformation. Before going any further, it is therefore worth briefly visiting each case study in turn to see what each says about the future transformations they hope to achieve, before getting into the main body of the chapter which will delve beneath these headline statements to see what kinds of narratives and concepts of time, futurity and change support them.

\subsubsection{Welsh Government}

The Welsh Government has been steadily strengthening its commitment to sustainable development since the 1998 Government of Wales Act (legislation which was influenced by global developments around sustainability at the time, namely the Brundland Commission's coining of the term "sustainable development" in 1987, the Rio Earth Summit in 1992, and the Treaty of Amsterdam in 1997, which saw sustainable development become part of the legal framework of the European Union). Its legislative commitment was increased in the subsequent Government of Wales Act (2006), making sustainable development a statutory duty of the Welsh Government, and in 2009 the One Wales: One Planet Sustainable Development Scheme of the Welsh Assembly Government confirmed that sustainable development would be the central organising principle of its administration. It remains one of just a few administrations in the world to have done this, and indeed this, as Matthew Quinn, director of Environment at the Welsh Government has commented, is a key part of Wales's "distinctiveness" in its devolution "journey". ${ }^{1}$ The One Wales: One Planet scheme $(2009,17)$ states that its vision of a sustainable Wales is one

where Wales lives within its environmental limits, using only its fair share of the earth's resources so that our ecological footprint is reduced to the global average availability of resources, and we are resilient to the impacts of climate change (see fig.3.1 for more)

In 2015, the Welsh Government's stance on sustainability was cemented through the introduction of a pioneering Well-being of Future Generations Act (WFGA) (2015).

\footnotetext{
${ }^{1}$ Matthew Quinn speaking at Swansea University on 22nd October 2015
} 
This Act places a duty on all public services in Wales to act in accordance with sustainability goals and ways of working specified in the Act, and has been accompanied, and shaped, by a nation-wide consultation exercise called The Wales We Want, involving around 7000 people. Public communication about the Act provided in the document Well-being of Future Generations (Wales) Act: The Essentials (2015, 3), states that the Act

is about improving the social, economic, environmental and cultural wellbeing of Wales. It will make public bodies think more about the long-term, work better with people and communities and each other, look to prevent problems and take a more joined-up approach. This will help us to create a Wales that we all want to live in, now and in the future. To make sure we are all working towards the same vision, the Act puts in place seven well-being goals.

The Welsh Government's vision is novel for a number of reasons. First, its stated aim to reduce the country's ecological footprint to a globally sustainable 1.88 global hectares per person, within the lifetime of this generation, marks a significant aspiration given that current levels of consumption equate to 3.28 global hectares per person. $^{2}$ Second, the policy innovations of the WFGA attempt to embed a longterm approach into Welsh politics, on the timescale of decades rather than the usual four to five year political cycle. Third, it promotes a notion of "intergenerational solidarity", which has recently been the subject of a United Nations report (2013) and recommendations, but very few national governments have enshrined the rights of future generations in their constitutions. Only a handful of countries have established national institutes or commissioners for future generations (they are Canada, Finland, Hungary, Israel, New Zealand, and Wales). Fourth, the Welsh Government emphasises the need to take an integrated, "joined-up" approach to transformation and sustainability, merging the environmental, social, and economic legs of the classic sustainability "stool" to form seven Well-being Goals (see fig.3.1), within which the Act also incorporates culture. It has therefore been recognised as one of the most holistic pieces of sustainability legislation worldwide. ${ }^{3}$ It's worth noting here that although the Welsh Government's climate change targets correspond to UK

\footnotetext{
${ }^{2}$ Ecological and Carbon Footprints of Wales Update to 2011 (Stockholm Environment Institute and GHD, 2015). Available at http://gov.wales/docs/desh/publications/150724ecological-footprint-of-wales-report-en.pdf. Last accessed 31st May 2017

${ }^{3}$ An assessment made by the World Future Council. Available at http://www.futurepolicy . org/equity-and-dignity/guardians/wales-well-being-of-future-generations-act/. Last accessed 31st May 2017
} 
and international frameworks, they are perhaps the least aspirational component of the Welsh Government's vision: its commitment to $3 \%$ reduction in greenhouse gas emission per year (Climate Change Strategy for Wales, 2010) are nowhere near the $69 \%$ annual reductions deemed necessary to avoid extremely dangerous warming of 2 degrees Celsius or more (Anderson and Bows 2008).

\subsubsection{Zero Carbon Britain}

The Centre For Alternative Technology's Zero Carbon Britain (ZCB) project's primary aim is to provide the research to show that a net zero-emissions society is possible with currently available technology, and to visualise what this future might actually look like. Since 2007 the group has produced scenario reports detailing how energy supply and demand in the UK could be managed with a $100 \%$ renewable energy system, changes in land use, diets, and lifestyles. ZCB's raison d'etre is summed up in a statement in its most recent scenario report, Zero Carbon Britain: Rethinking the Future $(2013,3)$ :

Closing the gap between current "politics as usual" and what is physically necessary to address climate change will require cross-sector collaboration and public engagement, framed by robust international agreements to foster high-level all-party political commitment. Zero Carbon Britain: Rethinking the Future provides a positive and technically feasible future scenario that aims to stimulate debate and catalyse action across all parts of society. Through this project, the Centre for Alternative Technology (CAT) hopes to inform, inspire and enable contemporary society to embrace the changes required to rethink the future.

Importantly, ZCB does not intend its research to be taken, as it says, as "eco-scripture that has to be followed to the letter", but is rather to open up conversations that "start with the realities that the scientific consensus demands" (Allen et al. 2013, 10). In contrast to the Welsh Government, ZCB therefore bases its scenarios on what current science deems necessary in order to stay below warming of 2 degrees Celsius, and its aim is to stir people's imaginations in this regard:

By getting people thinking differently about the future, we hope to catalyse urgent action across all sectors of society if we can't picture a solution, we will surely stay stuck in the problem. (Allen et al. 2013, 10) 
Our Vision of a Sustainable Wales is one where Wales:

- lives within its environmental limits, using only its fair share of the earth's resources so that our ecological footprint is reduced to the global average availability of resources, and we are resilient to the impacts of climate change;

- has healthy, biologically diverse and productive ecosystems that are managed sustainably;

- has a resilient and sustainable economy that is able to develop whilst stabilising, then reducing, its use of natural resources and reducing its contribution to climate change;

- has communities which are safe, sustainable, and attractive places for people to live and work, where people have access to services, and enjoy good health;

- is a fair, just and bilingual nation, in which citizens of all ages and backgrounds are empowered to determine their own lives, shape their communities and achieve their full potential.

To achieve this, sustainable development (the process that leads to Wales becoming a sustainable nation) will be the central organising principle of the Welsh Assembly Government, and we will encourage and enable others to embrace sustainable development as their central organising principle.

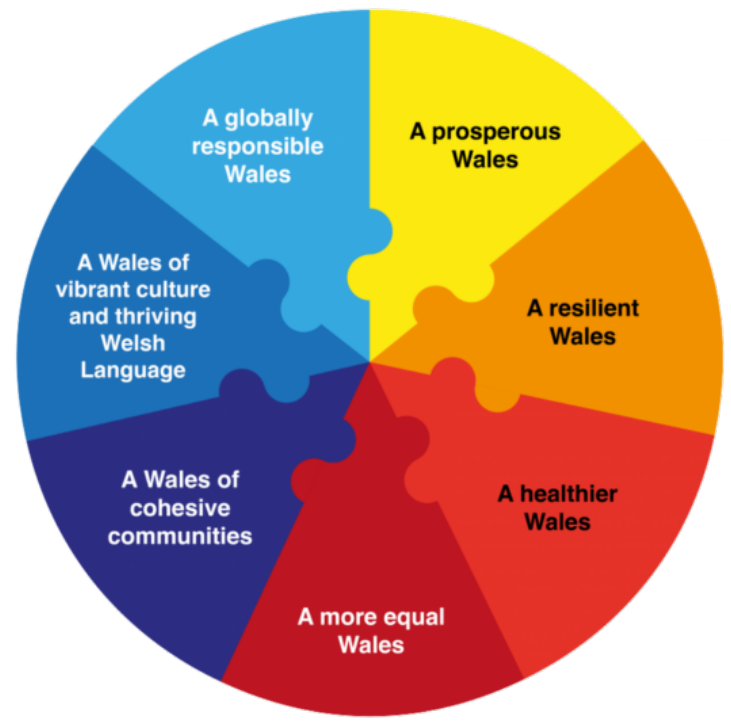

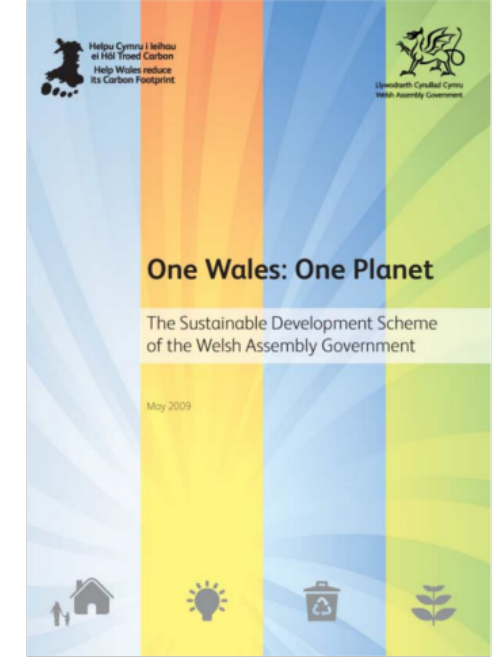

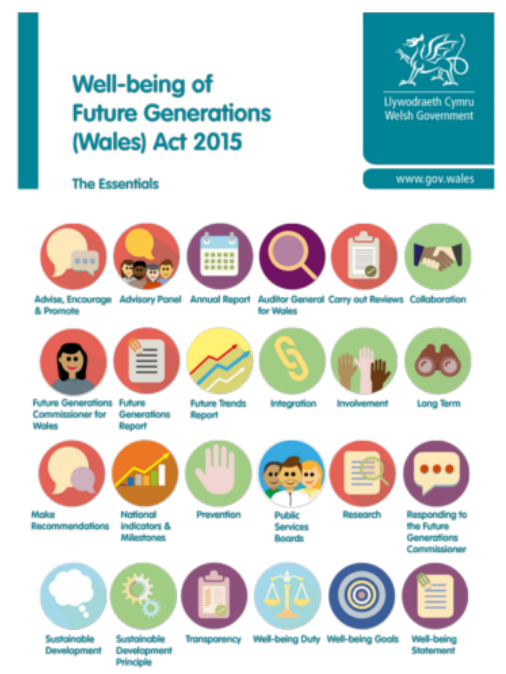

Figure 3.1: Welsh Government Sustainability initiatives: (top row) extract and front cover of the One Wales: One Planet Sustainable Development Scheme 2009 and (bottom row) the Well-being Goals as represented in public communications about the Well-Being of Future Generations Act 2015, and front cover of public guidance about the Act (The Essentials). 
ZCB, as its name suggests, has a Britain-wide focus, and it pro-actively communicates its research to a wide range of groups, from policy organisations to faith groups, nongovernmental organisations to Members of Parliament. This has extended to include international networks and the United Nations Conference of Parties on Climate Change, where ZCB has presented its research on several occasions. In Wales, ZCB is based at the Centre for Alternative Technology, itself an iconic institution in the environmental movement, and much of ZCB's public outreach revolves around talks and displays for CAT's visitors, and to school groups. In addition, Paul Allen, ZCB's project Co-ordinator, is a member of Wales's Science Advisory Council and serves on the Climate Change Commission for Wales.

\subsubsection{Emergence}

Emergence takes a more values-based approach to socioecological transformation. On its website, ${ }^{4}$ it states that

Emergence consciously seeks to embody the values we treasure in a hope that a more creative, caring and compassionate planet might be our next evolution, revolution, or re-evolution. Emergence is a collaborative project that advocates creative practice for a sustainable future through hosting artful events and gatherings.

In some ways this vision of transformation sounds vague in comparison to both the Welsh Government and ZCB, but this "openness" is very much part of what Emergence aims for. Its website goes on to describe Emergence as "a creative project and growing network which itself strives to work emergently", seeking to develop ways of working together which "recognise a more relational and dialogic approach."

Emergence's vision for transformation is radical in that it questions some of the ideological foundations of society, describing itself as both "a 'midwife' to new ways of being, creating and doing and a 'hospice worker' to an old paradigm no longer fit for purpose". These new ways of being, creating, and doing amount to what it calls "the art of living within the ecological limits of a finite planet."

\footnotetext{
${ }^{4}$ Available at http://www.emergence-uk.org/\#the-emergence-vision. Last accessed 31st May 2017.

${ }^{5}$ Available at http://www.emergence-uk.org/\#the-emergence-vision. Last accessed 31st May 2017.
} 
Initially, Emergence sought to reach a wide range of arts practitioners in Wales, but increasingly its activities have become "closer to home", involving a range of events, usually centred on activities of walking and/or talking, based in or around Swansea. Sometimes these events are tiny just a handful of people in a living room and at others they have involved upwards of one hundred people in public spaces. Depending on the scale, news about Emergence's events are spread either informally through networks of friends and acquaintances, or via more public advertisements on social media, Emergence's website, and occasionally on paper flyers which are distributed around public places by volunteers.

\subsection{Imagine that: attitudes towards change and uncertainty}

The concept of the absolutely new raises many anxieties. While it is clear that newness, creativity, innovation, and progress are all terms deemed social positives, the more disconcerting notion of unpredictable, disordered, or uncontainable change the idea of chance, of indeterminacy, of unforeseeability that lurks within the very concept of change or newness, seems to unsettle scientific, philosophical, political, and cultural ideals of stability and control. (Grosz 1999, 6)

"This Changes Everything", the title of Naomi Klein's recent bestseller about climate change, seems to sum up a general mood which surrounds the issues of environmental change and how humans should or might respond to it: that something, somehow, has to change. What makes this vast statement all the more slippery is that, simultaneously, everything is changing all the time, everywhere, and at speed. Even if we don't feel this in our everyday lives, we are readily reminded about the shape-shifting nature of the world by those who study it (e.g. Bauman 2000, Virilio 2006) and by a fast-paced global media. Change is inevitable, but how we engage with the idea of change is particularly pertinent to the issue of socioecological transformation, where the necessary changes are deemed to be so large: the recommendations of the Fifth Assessment Report of the Intergovernmental Panel on Climate Change, for example, calls for timely and significant society-wide transformational change (Gillard et al. 2016).

Change, however, is usually accompanied by uncertainty. Indeed "uncertainty" is 
frequently claimed to be one of the hallmarks of contemporary societies (e.g. Bauman 2007). In this section I hope to show how uncertainty, although often the source of much anxiety, can be engaged with in a range of ways which have implications for the kinds of transformations imagined by the organisations in question.

\subsubsection{Resilience}

Sustainable development provides us with the route to developing a sustainable and strong economy that operates within environmental and financial limits, which meets the needs of all our citizens now and in the future, and is resilient to future change (One Wales: One Planet 2009, 5)

A resilient Wales: A nation which maintains and enhances a biodiverse natural environment with healthy functioning ecosystems that support social, economic and ecological resilience and the capacity to adapt to change (for example climate change). (Goal 3 of the Well-being of Future Generations Act, 2015, 6)

One response to uncertainty is, almost intuitively, to try to guard against it as much as is possible. Resilience emerges as a key framing in the Welsh Government's approach to sustainability transformations, as the two extracts above highlight.

Resilience is a notion which has taken off over the past several years and can be found in a whole host of political discourses, practices and academic debates (Pugh 2014), particularly in relation to climate change and adaptation, from regional to global contexts (O'Hare and White 2013). As Simon and Randalls (2016, 4) suggest, resilience is "being offered as the solution to incredibly challenging societal problems and a key organizing concept in the zeitgeist of uncertainty". And yet "resilience" like "sustainability" is a notoriously vague and fuzzy term, utilised and mobilised in a broad variety of agendas. The Welsh Government's publications and communications regarding sustainability are peppered with the word "resilience", but little is offered in the way of a definition or specific applications. This ambiguity seems to be key to the term's apparent success; it tends to be passively received as a broadly helpful concept "upon which a host of strategies may converge to help society and cities better prepare for a range of risks" (O'Hare and White 2013, 275). Resilience names a seemingly positive future (after all it seems counter-intuitive to argue that we should not become more resilient to potential shocks or stresses) and yet it makes 
no promises (Simon and Randalls 2016). Nonetheless, Simon and Randalls (2016) maintain that there is, at least, one common theme across the diverse ways in which resilience is engaged with, and that there is a certain notion of flexibility and possibility. In one sense this feels appropriate: the postmodern era has been characterised by conditions of uncertainty, accelerating change and complex, perpetually-in-flux global circumstances (e.g. Bauman 2000, Tomlinson 2007); Bauman (2000) uses the term "liquid modernity" to describe these conditions of fluidity, constantly changing circumstances, and a propensity for "lightness" and mobility. Under such conditions, resilience seems to offer a tempting tool with which to stay afloat and navigate this tide of perpetual change, a way of shifting with the sands, rather than being swallowed up by them.

At the same time, however, the notion of resilience can be used to imply a certain impression of enduring and staying put. As a concept it

smoothly combines meanings derived from physiology (the capacity of material to return to a previous state), psychology (the capacity of an individual to return to normal after a traumatic event), ecology (the capacity of systems to continue functioning and renew themselves after a disruptive event) and informatics (the capacity of a system to keep on functioning despite anomalies and design flaws). (Aradau and Van Munster 2011, 46)

In this context, "resilience" is underpinned by an assumption that the present conditions ought to be maintained. Indeed, in contrast to social movements which welcome radically different futures that genuinely surprise, anticipatory politics aim to ensure that "no bad surprise happens" (Anderson 2010, 782). While this might indeed be desirable in terms of seeking to avoid potentially catastrophic effects of climate change, and to maintain Holocene-like climatic conditions, it is not so useful if resilience serves as a concept with which to preserve the political, economic and social arrangements which are creating environmental and social crises in the first place (e.g White and O'Hare 2014, Gillard 2016). If resilience implies the ability to "field", "absorb" or "bounce" back from extreme events in such a way that life can go on as before (Braun 2014, 56), then Walker and Cooper (2011) suggest that there is an "ideological fit" between resilience logics and neoliberal philosophies, as do MacKinnon and Derickson (2013), who write that "resilient spaces are precisely what capitalism needs spaces that are periodically reinvented to meet the changing demands of capital accumulation an increasingly globalised economy" (MacKinnon 
and Derickson 2013, 254). Genuine socioecological transformation and "resilience" would therefore seem to be quite at odds with one another, because resilience begets responses to the environmental predicament that are organised within the horizons of a liberal-capitalist order (Swyngedouw 2013b). The concern is that, despite the potential of "resilience", as claimed by some resilience scholars, to usher in new ways of thinking about human environment relations in terms of complexity, non-linearity and non-equilibrium (Gillard 2016), in practice resilience often serves to ensure that the neoliberal order can survive somewhat longer.

The Welsh Government's use of terms such as "building resilience" and "[becoming] more resilient to the consequences of climate change impacts" feeds into a corresponding narrative which enacts the future as a threat. For example, its One Wales: One Planet Sustainable Development Strategy $(2009,14)$ states that "climate change threatens the basic elements of life for people around the world access to water, food production, health, and use of land it also threatens our wider environment". As Anderson (2010) contends, across many domains of life, the future is increasingly problematised as a disruption or surprise, something to be preempted and prepared for, and liberal democracies are forging atmospheres and imaginaries of anticipation in relation to the future. Groves (2016) suggests that the future becomes charged with emotion and affect it is not simply anticipated, but anticipated with anxiety. Neocleous $(2012,187)$ claims that "trauma has come to govern us", and asks what this might be doing, ideologically and politically. Neocleous's central argument is that the idea of an "age of anxiety" is being constructed around us, so as to become an unquestioned part of our cultural common sense. The concerning thing about this is what Marx long ago spelt out: that capital, as a system rooted in feelings of everlasting uncertainty, both generates and thrives on anxiety (Neocleous 2012, 192).

This has implications for the ways in which futures are disclosed, related to, and governed. Braun (2015a) suggests that there has been a shift in the ways in which the future has been related to (at least in Western cultures), describing how whereas "in modernism time was seen to flow from the present to the future, today we increasingly experience time coming towards us, from the future to the present" (Braun 2015a, 239). Beck (1999) has characterised today's proliferation of anticipatory actions as a "world risk society", but points out that today's risks are calculated in relation to what is essentially unknown about the future, as opposed to the pre-modern known dangers which would have haunted the lives of people in the past. This marks a significant shift from a situation in which dangers such as plagues and natural disasters were understood as strokes of fate, attributable to Gods or Nature, to a far more 
politically charged context in which today's "risks" are intimately linked with human decision-making, accountability and responsibility. The concept of the Anthropocene, and associated ideas such as planetary boundaries and safe operating spaces, are increasingly linked with narratives of resilience and security (Randalls 2015), and the reconfiguration of public life around temporal registers of uncertainty, adjustment and repair (Barnett 2015). Acting in advance of the future particularly when it comes to climate change is an integral feature of life, and yet the ways in which it happens are rarely scrutinised (Anderson 2010). If the resilience category relies on an anxious political psyche, always preparing for an "oncoming attack" (Neocleous 2012, 192), then this legitimises particular kinds of governance and knowledge production, conditioning how "the future" can be intervened in, by whom, and with what objects of concern in mind. A host of measures, registers, apprehensions, engagements and movements are involved in making the future present through anticipation, and which lend particular weight to ideas such as "preparedness" and "prevention", and, especially, prediction. For example, the WFGA (2015) requires Assembly Ministers to produce a Future Trends Report twelve months before every Assembly election. The report includes predictions of likely trends in social, economic and environmental indicators in Wales. Justifying this move, the Welsh Government $(2015,10)$ states that

It's important that we understand the challenges that we will be facing, and have a clear picture of where we are heading.

In addition, it has devised a suite of National Indicators in order to measure progress towards the Well-being Goals. Ministers set milestones in order to establish expectations and chart progress. Regarding the Future Trends Report, the then Future Generations Commissioner for Wales, Peter Davies, told me in 2015 that

... corporate organisations undertake risk assessments in terms of what are the risks that the organisation is facing and in some respects for me the Future Trends Report is um, part of our risk assessment as to, you know, what are the external factors that are going to be impinging upon our ability to, you know, create the Wales ... we want. Um, some of which we can work with, positively, as opportunities, but some of which are trends or things that happen to be managed and that we have to build resilience ... to respond to.

The Welsh Government's framing of sustainability transformations in terms of risk- 
management reflects a wider tendency to reduce climate imaginaries to cost/benefitstyle analyses (e.g. Shaw and Nerlich 2015), a kind of "climate reductionism" (Hulme 2011) which focuses predominantly on impact and prediction rather than more imaginative accounts of social life and visions of the future. This particular gaze on tomorrow can permit recasting future social and environmental issues as technomanagerial issues of today, with science and technology held up as keys to adaptation and resilience. In the course of such reductionism, for example, "the problem" (which, in reality is complex, nebulous, messy) appears to crystallise around particular objects of concern. In what Beck $(2010,263)$ calls the "technocratic iron cage of environmental politics", $\mathrm{CO}_{2}$ is the "thing" around which which environmental dreams, aspirations, and policies crystallise (Swyngedouw 2013b). This can be seen in the Welsh Government's emphasis on particularly material aspects: transformation is framed largely in terms of efficiencies and reductions in resource use and $\mathrm{CO}_{2}$ emissions ("using resources efficiently and proportionately" is the phrase used in Goal 1 "A prosperous Wales" of the WFGA). This kind of focus, according to Žižek (2011, 353), results in a form of governance which sees "power turned into administration, relieved of its radical responsibility." Sywngedouw's description of this situation certainly resonates with WFGA. He sees administrative governance (associated with "commodified" entities such as $\mathrm{CO}_{2}$ ) as constituting "a set of managerial and institutional technologies that revolve around reflexive risk-calculation, self-assessment, interest-negotiation and intermediation, accountancy rules and accountancy based disciplining, detailed quantification and bench-marking of performance" (Swyngedouw 2013b, 5).

\subsubsection{Vignette: Swansea Council and the future}

Swansea Council offers a closer insight into the Welsh Government's anticipatory orientation to the future, particularly how it manifests itself at local scales of governance. Swansea Council happens to be my Local Authority, and has been earmarked as the flagship council for sustainability in Wales by the Welsh Government. My analysis of several of the council's strategic documents (including a pilot Future Trends report for the Local Authority) is complemented by in-depth interviews with three of the four members of the Council's Sustainable Development Team. I also participated in a workshop run by the Sustainable Development Team, which introduced the concept of Scenario Planning to other Council staff.

Talking with Council staff and reading its strategy documents gives one a sense in 
which the future is being "geared up for". It is constructed as a matter of getting organised, of planning, route-mapping, meeting targets and working together. At the time of my research with the Sustainable Development Team, the Well-being of Future Generations Act had just been passed but councils had yet to receive guidelines as to exactly how the Act would be implemented, and thus it loomed large on the Team's horizon. This sense of anticipation and uncertainty around the implications of the Act was compounded by a language which enacts the future as a threat, which seems to have been introduced into the Local Government lexicon by a piece of work by an academic consultant which was commissioned by the Welsh Local Government Association in 2008. Futures, Trends, and Horizon Scanning for Welsh Local Government (2008) was intended to help local authorities to incorporate futures issues into their planning and service delivery. The document frames the future largely in terms of risks, threats, and shocks from climate change to demographic change and presents a range of quantitative predictive data, as well as an introduction to several tools that staff can use to better take into account these trends, such as horizon scanning, trend analysis, and futures workshops. The report is highly deterministic in its tone, and begins one of its introductory chapters with the paragraph

A review of the futures literature has provided an overview of the key trends and drivers which will influence our communities and way of life in the coming decades. There is a consistency of message from much of this material, backed up by statistics and scenarios which suggest a range of threats, risks, challenges and opportunities for society as a result of these 'drivers'. Public authorities will have to react to these events.

It continues

Futures analysts suggest that "Intelligent" strategy will consider the future trends which may favour, allow or threaten well-being and seek to make and take opportunities to contain and manage threats. It will also consider the level of impact, the likelihood, symptoms and causes of trends and also consider "whether we are on the right track" and "are we moving fast enough" to meet out aspirations given these influencing factors on our communities.

In turn, this language is reflected not only in the Well-being of Future Generations Act (particularly in the legislation's emphasis on progress reporting and future-trend reporting), but also in Swansea Council's own Future Trends for Swansea report 
(2015), which was based on the academic consultant's recommendations. Its report (which has been circulated amongst other council departments) begins

This paper is a regularly updated working document that offers an insight into our current understanding of future trends likely to impact the planning and delivery of Council Services. The trends identified are based on sound evidence and statistics referenced from credible sources of intelligence. However the information presented is not absolute or certain but rather intended to provide a reflection of wider visions of a probable future based on current drivers. Trends have been sourced from a variety of global, national and local contexts and were selected for the relevance of the impact on City and County of Swansea Services.

My impression is that the future is thus constructed as something to be informed about, not something to be created, or even yet to happen. Although the documents are essentially exercises in detailing some possibilities and likelhoods, in effect they create a much more fixed idea about the future as already determined. For example, one staff member (A) told me that the Future Trends for Swansea document amounted to

a long-term vision, so these are the issues that are likely to face the overarching issues we're likely to face in Swansea this is what we're going to do now to prepare for that ... in 2040

Another staff member (B) told me that one question the team therefore asked themselves after they had produced this Future Trends document was

how can we inform local residents about the future, from this foresighting document?

In this approach to the future, much emphasis is placed on external "drivers" and potential impacts, rather than the agency of people themselves. For example, staff member (A) told me

we've produced a future trends paper which ... has various different trends which are kind of they're more scientifically based rather than looking at scenarios or looking at different views or foresighting as such? It's kind of we're looking at more ... evidence-based things to put in our documents. Um, not looking at views [or] what people think, specifically. 
There is therefore a sense of powerlessness or defensiveness in the face of global trends, particularly with respect to the economy, technology, population, and climate change. It is interesting to note which trends have been selected and how they are subsequently framed. In the Future Trends for Swansea document, as with the preceding Futures, Trends and Horizon Scanning for Welsh Local Government (Netherwood 2008), the trends selected are overwhelmingly negative or threatening in some way from the pressures of globalisation to the possibility of food scarcity. Importantly, although these are speculative documents, the trends are couched in a language of what will happen as opposed to what could happen further creating a sense that the future is already determined. For example, the Futures, Trends, and Horizon Scanning report features predictions for the "Big picture" which includes statements such as

Enhanced by IT, expectations for quality of life, goods and services will be higher. Secularism and materialism will grow, with capitalism continuing to be the dominant force in our lives. Gender equality will continue to grow in the developed world. There will continue to be a decline in civic values ... (Netherwood 2008, 7)

And yet, as Hebdige (1993, 270) contends, "nothing is ever bound to happen" although representing the future as such has particular ways of structuring potentialities and thinking possibilities.

The desire for evidence about what this "inevitable" future has in store corresponds with a framing of uncertainty as a key barrier or blocking point for transformational change (which also needs to be understood in the context of generalised uncertainty about the the prospect of job losses and funding cuts to Local Authorities in the coming years). As the Sustainable Development team leader explained in the scenario exploration workshop in 2015

when we [the Sustainable Development team] have approached people about this the reaction has been "no, we can't, too far ahead, too uncertain!" So, we are using scenarios to help overcome this blockage...

Scenario planning, and the associated concept of "horizon scanning", implies an ability to look ahead, as if in a watchtower, to see what's coming, to reduce uncertainty and to have time to prepare for it. Implicit in this is a linear imaginary of time and a way of relating to the future as almost a physical realm out there, ahead. One can detect a sense of "holding the fort" in this kind of imaginary, a sense almost of embattlement which is heightened by the ways in which staff members talked about 
the need for unity and coherence, to embed and align with the Well-being Goals, and also their use of language regarding understanding strategic risks, building capacity and increasing resilience.

Just as with the wider Welsh Government narrative, however, there is a tension with this linear imaginary which seeks to predict and resist the future, with another one which forges ahead with a language of progress, of achieving targets and creating, as Swansea Council's Corporate Strategy states, "A safer, greener, smarter, fairer, healthier, richer Swansea achieved, in part, by ensuring we have a sharp focus on the delivery of our commitments" $(2015,4)$. In addition, the language of the Welsh Government's One Wales: One Planet Sustainability Strategy is echoed in Swansea Council's One Swansea Plan, both of which emphasise the importance of working together towards a shared goal.

On reflection I am left with a sense of two narratives vying for attention in Swansea Council from above there are the visionary goals set out by the Welsh Government, reflected in the corporate language and public communications of the Council management. But behind the scenes there is a rather more embattled feel to the Council's relation with the future, where the future is constructed as a threat to be prepared for. Time, resources, and uncertainty regarding long-term council funding and jobs create an atmosphere in which there is barely enough energy to prepare for what are deemed inevitabilities hurtling towards them, let alone to imagine and create a future of their own making.

To conclude this section on the Welsh Government and Swansea City Council, it is worth returning to the point that anticipation, and, specifically, resilience, are on the face of things difficult notions to find fault with, as they point to a vague yet seemingly optimistic aim (White and O'Hare 2014). And yet it is precisely this "common sense", consensual, framing which is questionable in terms of imaginative capacities for transformation. Nordmann (2014), for example, argues that "if we think of the future as something to be anticipated, expected, prepared, or braced for, and, at best, modulated as it comes upon us, we postulate ourselves as fundamentally unfree with respect to the future" (Nordmann 2014, 93). In contrast, he suggests that an ability to "freely envision a future world that accords to our values and needs" (ibid.) is what is needed in order to break the mould of anticipation which reproduces neoliberal horizons, allowing us to judge scenarios according to desirability rather than inevitability. In the next section, I turn my attention to another case study, Emergence, and its rather different treatment of uncertainty, in order to elucidate 
how such an approach might take shape.

\subsubsection{Embracing uncertainty}

How is it possible to revel and delight in the indeterminacy of the future without raising the kind of panic and defensive counterreactions that surround the attempts of the old to contain the new, to predict, anticipate, and incorporate the new within its already existing frameworks? (Grosz 1999, 16)

If the Welsh Government seeks to deal with uncertainty through particular registers of preparedness, prediction, resilience, and anxiety, Emergence deals with uncertainty in very different ways. In Emergence's approach, uncertainty and instability are welcomed as sources of transformative capability, rather than something to be guarded against or prepared for. Intriguingly, this approach shares some ground with resilience thinking in terms of an orientation towards "complexity" science, which understands matter as made up of networked connections, evolving in often unpredictable and non-linear ways (Gillard et al. 2016). However, whereas the Welsh Government's approach tends to approach this complexity through consensual management and administration, Emergence sees the discord and instability of such complexity as that which provides the most promising openings and opportunities for change. Thus, on its website Emergence describes how it

recognises that the world in which we live is one which itself has emerged from countless processes, decisions, policies as well as intended and unintended actions. We recognise that the outcome of our actions is unknown and there is no one grand solution or magic widget capable of fixing everything that is broken. Everything is interconnected. The threads that comprise the web of life run through each of us and connect all our actions for good or ill.

Emergence's acceptance of uncertainty in the face of what it believes to be fundamentally unknowable marks a striking contrast to widespread political commentaries which have embedded within them a supposed desire for security, certainty, and control (Neocleous 2012). The Welsh Government's use of the term "resilience" can be seen as part of a wider constellation of discourses which feed off and into this assumed desire. At the time of writing, for example, the British Prime Minister, Theresa May, 
had used the election campaign slogan "strong and stable leadership" so much that it had become the subject of widespread national ridicule and satire. Emergence's approach, by contrast, rejects this assumed need for absolute certainty and security, and in fact it welcomes a sense of vulnerability in light of inherent uncertainty.

The word "vulnerability" usually has negative connotations in relation to environmental change: vulnerability is something to be avoided, protected against, forestalled. Nonetheless, a growing body of work suggests that being able to grasp complexity (and part of developing ecological sensitivity) is itself a matter of acknowledging our own vulnerability to such complexity (e.g. Macy and Johnstone 2012). It is in this spirit that Emergence has made the embracing of vulnerability an essential part of its activities, particularly through nurturing spaces for open, non-judgemental conversations.

In 2015, for example, Emergence organised events (called COP123) over three consecutive Saturdays in Swansea to mark the COP21 climate talks taking place in Paris during the same period. Each of the events was designed to allow the space and time six hours in fact for people to talk about their reactions to screenings of climate films (Chasing Ice, Age of Stupid, and This Changes Everything, respectively). Rather than seeking to raise awareness, or galvanise action, Emergence's intention was rather to allow space for the feelings associated with the environmental crises depicted in the films attention to which is often pushed aside in favour of doings to be voiced. A conversation becomes a way of allowing vulnerability into the room, although this is still not easy within a frame of social norms that prioritises strength over weakness and fear. At the first COP123 event, for example, participants initially broke into excitable (sometimes confrontational) exchanges about the root causes of, and solutions to, climate change. It wasn't until Emergence's co-director Philip Ralph performed his improvised monologue One Eyed Man (fig.3.2), in which he lays bare his own fears and vulnerabilities in relation to worldly phenomena, that the atmosphere changed and people began to voice their own feelings about climate change. Ralph's intention with these performances was not to hide behind a character, but to talk candidly about his thoughts, feelings, and uncertainties a process which often evolved into a conversation with the audience. At all three COP123 events, the effect of his honesty and openness was arresting, and created an atmosphere in which people felt similarly inclined to be open and honest about their vulnerabilities in their subsequent conversations with one another. As Ralph explained:

The elephant in the corner of all of this, for me, is fear. Which has been 


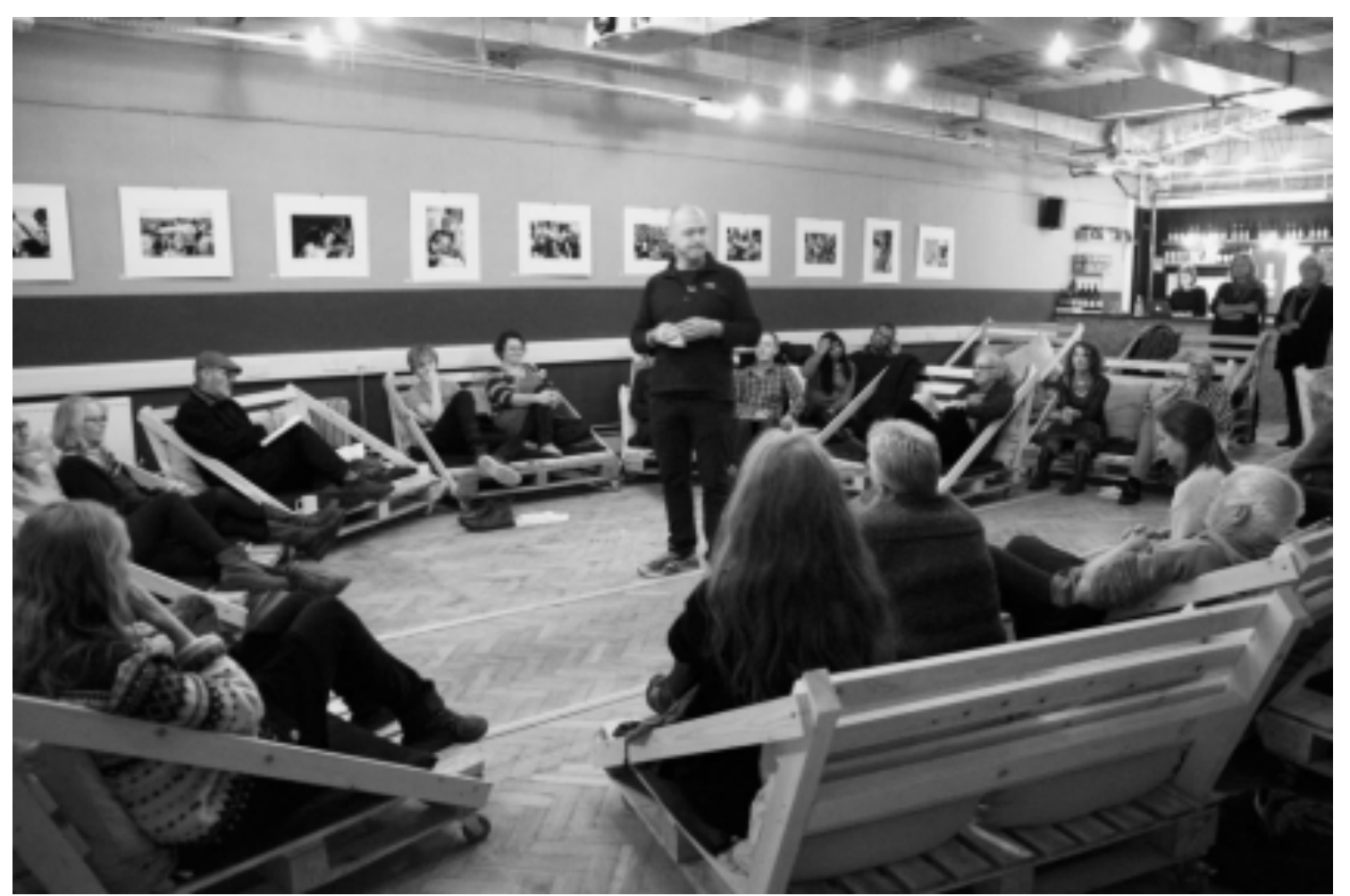

Figure 3.2: Philip Ralph performing One Eyed Man with participants at COP123. (Source: Eleanor Flaherty)

rife this week [the week in which terrorist attacks shook Paris in November 2015]. But, I would argue for me, don't know about you is rife all the time now. I'm afraid ... to turn on my computer, I'm afraid to look at the news, I'm afraid to go out on the street, and talk to somebody. I'm afraid to have these conversations in rooms with people who I don't necessarily know what they feel.

Dewsbury (2003) contends that bearing witness to what is happening in the world is a necessary prerequisite to changing it, and is sometimes the hardest part, for it requires transgressing the stage of representation, "creating a space to think where there is no model for action, emphasizing the world's unspoken demands in the present more than its prescripted futures" (Dewsbury 2003, 1908). Paradoxically, "staying with the trouble" (Haraway 2016), rather than jumping to solutions, can facilitate a deeper engagement with socioecological crises and possibilities for change. Fern Smith, speaking at an Emergence event in 2015, echoed this sentiment, saying

to get to a place of action we have to go through a sense of how we feel about stuff ... we need to go to the place of difficulty ... and see that other people are there too. 
Emergence frequently uses the notion of "holding a space" in which these kind of vulnerabilities can be shared. In order to do this, it often draws on methods of Council (Zimmerman and Coyle 1996) as a way of facilitating group discussion, and asks that people try to speak and listen "from the heart". Usually, a talking piece a smooth, heavy, palm-shaped rock is passed around the circle to signify whose turn it is to speak, and a candle is often placed in the middle of the circle. These sensory encounters help to create a space where the spoken word is honoured and where people feel accepted and listened-to. While this might sound romantic, its ritualistic ambience nurtures, and requires, absolute trust and openness and negates the urge to premeditate what to say. This can be a profoundly visceral and often uncomfortable experience. People respond to method of Council in different ways, with laughter, tears, and embarrassment, as Reason (2007) also recalls, but it is nearly always powerful.

Ramsden (2017) observes how the I-don't-know space of vulnerability in a conversation between strangers can be a site for generating ethical awareness: voice can be thought of as an ethico-political force capable of creating worlds and opening up spaces for different ways of perceiving and being together in the world (Kanngieser 2012 ), often in ways not possible through other forms of communication. For example, during COP123, I, too, found that I voiced to virtual strangers thoughts and feelings about climate change that I don't even discuss with close friends and family; largely, I realised, for fear of making myself and others uncomfortable. My impression was that other participants found the COP123 events to be similarly cathartic experiences. Months later, for example, I bumped into one participant and, reminiscing, he referred to the events as having been being "soul food".

In conversation with me, Fern Smith referred to the concept of "negative capability" as something which underpins her own approach to uncertainty, and which informs the work that Emergence tries to do. Negative capability is a phrase first used by the Romantic poet, John Keats, in a letter to his brother in 1817. In it, he writes that negative capability describes how a person "is capable of being in uncertainties, mysteries, doubts, without any irritable reaching after fact and reason" (Keats 1899, 277). Numerous writers have taken up Keat's idea of negative capability, including the social theorist Roberto Unger, who adapted the term to explain how people can be empowered against social and institutional constraints, and to deny any fixed schemes of division, hierarchy, and routine (Unger 2004). Dewey also refers to Keat's insight to suggest that the "silent workings" of the imagination, including elements of chance, spontaneity, and intuition are sometimes closer to understanding "truth" 
than any amount of reasoning (Dewey 1934, 33).

It perhaps seems wholly unreasonable for me to even attempt to compare the Welsh Government's and Emergence's approaches to socioecological transformation given the hugely different contexts and responsibilities each has the Welsh Government, after all, is tasked with running a country (with all the socio-historical-political constraints and "baggage" which comes with that), while Emergence is a far more autonomous organisation in terms of its freedom to experiment and (far smaller) responsibilities. Gillard et al. (2016) suggest that, in the context of environmental policy, it can be expected that macropolitical structures produce incremental or "of a kind" change, whereas micropolitical entities (personal networks and social movements, for example) can produce radically alternative discourses and practices (see also Inayatullah 1990). Nonetheless, given the centrality of uncertainty to the experience and organisation of everyday life, particularly with regard to the future, it is perhaps not too ambitious to ask how politics might look if it were to engage with uncertainty differently. As Grosz $(1999,11)$ asks, "what, for example, would politics be like if it were not directed to the attainment of certain goals, the coming to fruition of ideals or plans, but rather required a certain abandonment of goals?" Philip Ralph, speaking at the launch of Emergence's Culture Shift Report, put it bluntly:

Saying you don't know something doesn't mean "I'm an idiot". The more that we play the game of "Hello I'm so and so and I'm in charge" the more we are, frankly, fucked. We want certainty, we want to know but wouldn't it be wonderful if politicians stood up and admitted [that] we are not sure?

Ralph's comments lament a political climate in which acknowledging uncertainty amounts to electoral suicide. And yet it is precisely an element of chance that signals, according to Grosz, the openness of the future, its relative freedom from the past, and the possibilities of paths of development. This is an out-of-joint time (in so far as it is out of joint with dominant relations to time and futurity) which cannot be contained in a model, plan, or blueprint, but is a positive leap into the unknown, of what we can't yet see or think is happening (Rajchman 1999). The ways in which uncertainty is related to, therefore, has implications for how the shape of the future is imagined. It is to this subject that I now turn. 


\subsection{Temporal trajectories}

\subsubsection{Linear time}

All three of the case studies discussed in this chapter the Welsh Government, Emergence, and Zero Carbon Britain contain within their approaches a certain sense of forward motion or orientation. The predictive orientation of the Welsh Government's anticipatory politics entails a linear conception of time, and this is reinforced by a tendency to employ language which underpins a sense of forward motion. As already mentioned, the WFGA is founded on seven well-being "goals", and the legislation includes "milestones" and "future trends" reports. In addition, the Welsh Government frequently refers to its legislation in terms of a "journey" (e.g. "This [One Wales: One Planet] Strategy is a critical step on a journey to meeting that bigger challenge"), "stages", "pathways" and "the road to achieving the vision". Phrases such as "working towards" and "route map [of the journey]" are also indicative of a particular way of relating to the future, and in particular conveys a sense of regulated transformation towards a particular aim. Zero Carbon Britain, too, frames the whole endeavour of getting to net zero carbon in terms of a story of forward motion and progress. Written communication material and presentations to the public (which I delivered during my time volunteering with ZCB), depict the sweep of human evolution and history, as encapsulated by the title of ZCB's presentation "The Extraordinary Story of Human Beings and Energy". Paul Allen, ZCB's project co-ordinator, told me that

we have to begin to plot what paths of rapid decarbonisation will get us down to the safe limit

Correspondingly, ZCB contextualises its work within timeframes often associated with climate change scenario planning, for example the years 2030 and 2050, and often uses trajectory-style diagrams to highlight how its scenario compares with those of other commitments on emissions reductions (fig. 3.3). The titles of its three most recent reports also exude an enthusiastic, directed stance towards the future: "Zero Carbon Britain: Rethinking The Future" (2013); "Who's Getting Ready for Zero?" (2015) and "Zero Carbon Britain: Making it Happen" (2017). Emergence, as already mentioned, also has at its core a sense of forward motion of emergence, evolution, and transformation, but, significantly, it does not evoke any particular goals or aims located on a particular temporal horizon. 


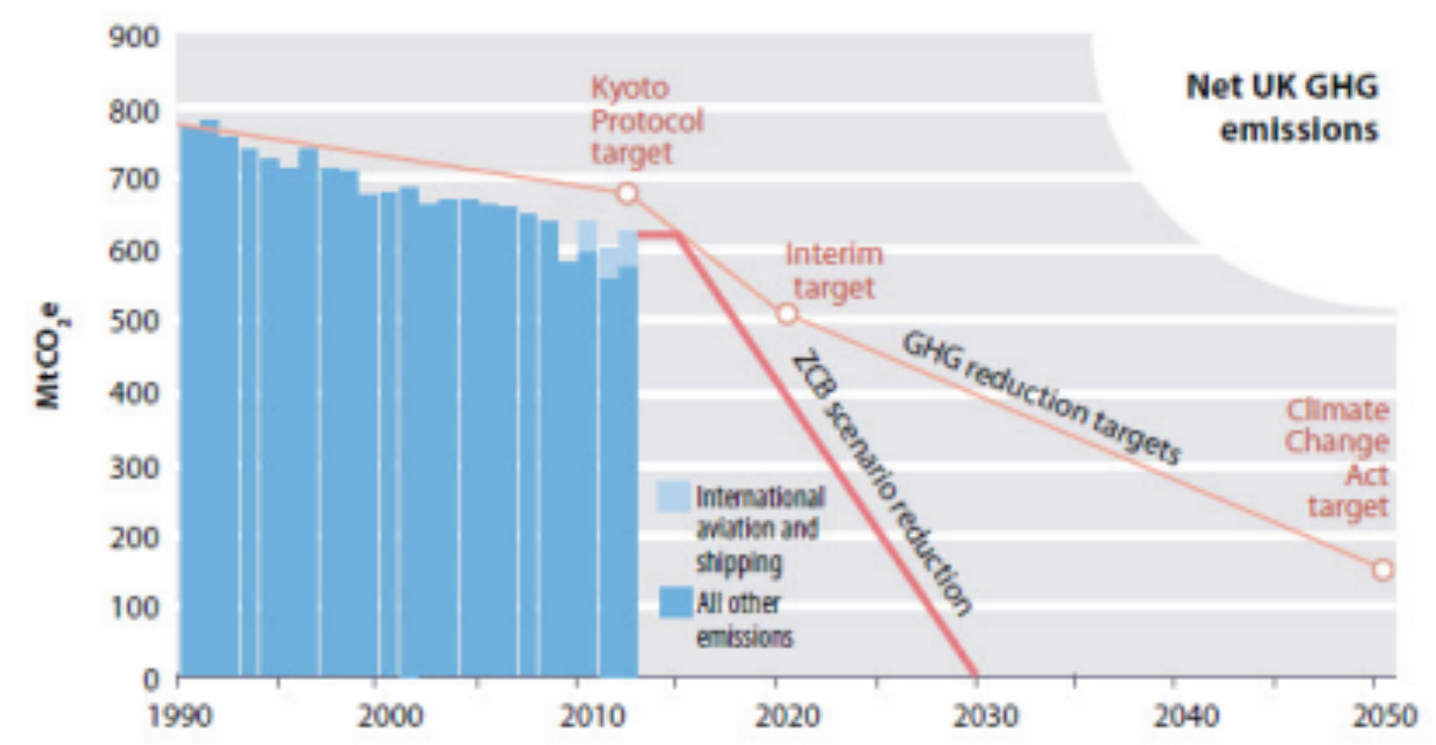

Figure 3.3: Graphic representation of the ZCB scenario trajectory.

The idea of progress and a sense of moving forwards towards the future (or, for that matter, backwards to the past), is a particularly "modern" imaginary the modern era is characterised by an orientation to the future which is invested with a collective sense of purpose and improvement, a sense of leaving the past behind (Bauman 1993). Compelling visions of the future are central to such imaginaries. Indeed, following various diagnoses of the postmodern condition as being marked by a loss of utopian thought about the future, and therefore a fading of any belief that there could be any alternative, many have argued that a resurgence of the utopianism of modernity is necessary (e.g. Harvey 2000, Levitas 2013). Proponents of so-called Green Modernity (e.g. Nordhaus and Shellenberger 2007) urge that aspirations for continuous improvement, innovation, novelty and progress are precisely the qualities which must be held on to if there is to be any hope of mobilising the political and social will (and energy) to transform to more sustainable arrangements. As Latour (2008) contends, the thrusting-forward arrow of time towards a picture of an attractive future, and its associated emotions of enthusiasm, frontier spirit and optimism, is unparalleled in its ability to unlock political passions, and to develop a politics of possibility necessary for overcoming the depoliticising effect of doom and gloom environmentalism. As Latour says, now is the time "to develop, not withdraw" (2008, 13). Hebdige (1993, 278) contends that the metaphor of the journey is "the most trite, overused, banal metaphor imaginable for the way we move through time", and yet it is undeniably powerful as a focus for collective as well as personal identification within historical narratives. The Welsh Government and ZCB appear very much to be buying into this 
sentiment. Indeed, their depictions of desirable future states towards which society can collectively travel seem to want to defy the shifting sands of liquid modernity and to regain some sense of the progress-orientated optimism of earlier modernity. In such a stance towards the future, Inayatullah $(1993,242)$ suggests, "humans are not left alone wondering aimlessly in a universe that has no certainty". Bauman adds that linear time is an imaginary more hospitable to life as a pilgrim, that is, orderly, predictable, determined, ensured. He $(1996,22)$ suggests that:

$[\mathrm{P}]$ ilgrimage is what one does of necessity, to avoid being lost in a desert; to invest the walking with a purpose while wandering the land with no destination. Being a pilgrim, one can do more than walk one can walk to. One can look back at the footprint left in the sand and see them as a road. One can reflect on the road past and see it as progress towards, an advance, a coming closer to; one can make a distinction between "behind" and "ahead", and plot the "road ahead" as a succession of footprints yet to pockmark the land without features. Destination, the set purpose of life pilgrimages, gives form to the formless, makes a whole out of the fragmentary, lends continuity to the episodic. (Emphasis in original)

And although Bauman's assertion is that this kind of approach to life is no longer available or viable in the present, post-modern or liquid-modern conditions, it is striking how the Welsh Government and ZCB seem to grasp at a pilgrim-like narrative. There is also something of a tension between this progress-orientated narrative and the resilience narrative outlined in the previous section. While the former appears to strive to transform the world anew, the latter seeks to preempt and prepare for a future which is seemingly beyond control. It is as though these two impulses pull in opposite directions along the same linear trajectory. This tension is especially apparent in the Welsh Government's narrative, and is compounded by a corresponding discourse of limits which it employs in relation to human ecological relations. In its ambition to achieve the goal of "an innovative, productive and low carbon society which recognises the limits of the global environment and therefore uses resources efficiently and proportionately" (WFGA The Essentials 2015, 5) as well as repeated references to "living within environmental limits" (a notion which is also employed by both ZCB and Emergence to some degree), the Welsh Government echoes a wider resurgence of interest in the notion of environmental limits (Rickards et al. 2014). Whereas Enlightenment/Modern attitudes to progress celebrated the transcendence of environmental limits, the environmental politics which emerged after the 1972 Club of Rome report Limits to Growth (Meadows et al. 1972), warned that growth 
was inescapably limited by the physical facts of existence, and urged that current trajectories of growth were no longer tenable. Despite recent efforts aligned with Green Modernity to re-vamp environmental politics to have a more forward-looking, optimistic feel rather than a conservative one (for example, the UN's World We Want project), notions of environmental limits have gained renewed credibility through the popular concepts such as "planetary boundaries" and "safe operating spaces" for humanity (Rockström et al. 2009). The Welsh Government, then, appears to be juggling two contrasting imaginaries of transformation: one which encourages progress and innovation; and one which calls upon society to "pull back" and limit itself.

Rather than trying to ascertain the particular pros and cons of either a progress or limits-orientated temporality, it is useful to step back and consider the kinds of imaginaries created by a linear conception of time more broadly. Romanyshyn (1989) describes how linear perspective initially developed as a technique in Renaissance art, where space was organised according to vanishing points on the canvas and located objects on the same plane, rather than the multiple perspectives evident in earlier Medieval art. Subsequently, this perspective became a pervasive "habit of mind" for western-Enlightenment societies, and was tightly bound to the emergence of technological worlds and to sociotechno-imaginaries (Jasanoff and Kim 2009). The emergence of linear perspective is significant because it is one in which "the observer is positioned as a spectator outside the space thus represented" (Groves 2016, 5). Thus, linear perspective creates a model of time in which subjects are always somewhat divorced from the future (the future is "out there"). Extropolating from this, it could be argued that the Welsh Government and ZCB are both creating imaginaries of time and the future which are always projecting forward to a time which is "not yet" for the Welsh Government, it is "future generations"; for ZCB, it is 2030. And so, while a future-orientated utopian imaginary is frequently held up as something that is necessary for radical social change (indeed, Bloch (1986) conceptualised the "not yet" as a latent form of hopeful anticipation which is central to human thought), the practice of envisioning future states is simultaneously criticised by some for the limitations it can place on people's own imaginative capacities. For example, and especially in relation to climate change, there is concern that people may find it difficult to conceptualise a time beyond that of their own lifetimes, and to imagine distant futures "end points" such as 2050, or several generations hence (e.g. Brace and Geoghegan 2011; Hulme et al. 2009). A related problem, identified by Falk (2016), is that this focus on the "not yet" encourages a politics of postponement and reinforces the feeling that climate change (for example) is a distant threat. Another criticism 
is that this perspective of the future as "a space of points and plotted trajectories" (Grove 2016, 5) can have the effect of closing down choices and justifying particular forms of power quite the opposite of the "politics of possibility" intended by proponents of Green Modernity (Nordhaus and Shellenberger 2007). In linear imaginaries, Inayatullah $(1993,250)$ contends, "time is largely reductionist with efficiency as the primary goal", and this legitimises, as with "resilience", a range of techno-managerial policy actions in the present which forestall the eruption of genuinely new ways of knowing and doing (Inayatullah 1990). One related effect is that agency for transformation is removed from the lives of "ordinary" people climate politics remains an "elitist and expert discourse" (Beck 2010, 254), depoliticising subjects because it transfers agency to centres of power (science, technology, and governance), and away from individuals and communities (MacKinnon and Derickson 2013, Inayatullah 1990).

Some have questioned whether a linear imaginary is capable of fostering the kinds of transformation needed, when more nuanced and complex understandings of society and ecology and transformation are warranted (e.g. Prigogine and Stengers 1997, Urry 2005a, Morton 2010). Inayatullah suggests that what is needed is a "multiple theory of time and space" $(1993,249)$, and he points to the existence of other temporalities and metaphors of time, including cyclical, spiral, and decline, perspectives. Such "heterotemporality" (Klinke 2013, 678) the existence of multiple temporalities alongside one another affords a richer imagination of time and transformation and corresponds more closely with the lived experiences of people, that is, time which appears to go slowly or speed up, time which stands still, time which repeats (as in the routines of our daily lives), not to mention time which surprises. As such, lived time tends to be experienced as spiral and fractal, rather than linear (see fig. 3.4), and incorporate multiple objects of concern such as other people, places, communities an "interdependence of linked narratives spiralling on through time" (Groves $2016,7)$. It is from within lived futures, rather than from abstracted perspectives on the future which flatten and linearise, Groves contends, that society will be better able to cope with uncertainty through relations of connection and attachment.

Although it is not my intention in this chapter to repeat long-standing debates in philosophy about the nature of time, it is clear that some of the basic assumptions about time which underpin the case studies I discuss here can be traced to some of these fundamental standpoints regarding time. The linear temporality outlined above, for example, aligns with a Newtonian view that time is absolute, and passes uniformly and predictably, independently of human life. This, as Massey (2005, 32) 
describes, is a time (and a future) "which is already given in the initial conditions". By contrast, an opposing view on the nature of time is encapsulated by Bergson's famous phrase "time is invention or nothing at all" (1998, 341), which rejects any kind of determinism and understands time as essentially unpredictable, and emergent through action. It is this idea of time which underpins Emergence's approach to the future, as I explore in the next section.

\subsubsection{Emergent time}

How to think of direction or trajectory without being able to anticipate a destination? (Grosz 1999, 9)

If the idea of linear progress towards particular goals is problematic, and yet if we accept the suggestion that it would be a good idea to transform in some way towards improved socioecological conditions, then how, as Grosz asks above, can one imagine such a trajectory without anticipating the destination?

The concept of "emergence" from which Emergence takes its name presents some possibilities in this regard. Emergence (the concept) derives from complexity theory (e.g. Holling 2001, Urry 2003, DeLanda 2006) which moves away from theories of equilibrium, and instead posits a non-linear conception of evolution and change which recognises that events do not always unfold in predictable ways, or according to an "action-reaction model" (Inayatullah 1994). In this context, emergence refers to the ways in which systems (be they cultural, environmental, economic systems) self-organise and learn, often resulting in very complex situations arising from relatively simple starting points. Conversely, emergence also refers to the ways in order "emerges" at the edge of chaos (e.g. Prigogine and Stengers 1984). In this sense, the phenomenon of emergence cannot necessarily be understood in its totality, it cannot be broken down into parts, because everything influences everything else, often in surprising and unpredictable ways. Rather than a deliberate, planned, and measured approach to change, there is a sense of something fundamentally unplanned at work. As Philip Ralph, Emergence co-Director explained at one Emergence event in 2015

We're connected not only in the fact that we are connected because we are all ... inhabitants of this planet but we are connected because we are all ... within this system emergence, the name of this enterprise, "emergence"... recognises that factors come together to create circumstances and actually, 
one cannot predict it, one cannot plan it and it just goes. And we're all part of that.

Emergence, and complexity theory, challenges deterministic ways of thinking about the future. This is especially noticeable in their handling of unpredictability. Whereas unpredicability is, as already discussed, a feature of resilience narratives, it seems that, often, while the resilience discourse acknowledges a complex-systems view of the world, it fails to extend it to ways of relating to the future. Rather, the unpredictability becomes a source of anxiety and the resilience narrative gets forced into a linear account of the future as a "space of plotted points and lines"(Groves 2016, 5). By contrast, the idea of emergence presents some possibilities for maintaining a sense of complexity and keeping hold of a notion of chance and openness in relation to the future. Emergence's interest in uncertainty, vulnerability and unpredictability is an approach that tries to welcome chance. In complexity theory, chance (as with uncertainty) is the motor of creative evolution (Bergson 1998). The work that Emergence (the organisation) does is concerned with how to take this understanding of time and transformation of the nature of the universe itself and find ways of working with it in social life. Emergence (the concept) refers to how time proceeds "not by continuous growth, smooth unfolding, or accretion, but through division, bifurcation, dissociation by difference through sudden and unexpected change or eruption" (Grosz 1999, 28). This echoes Althusser's "aleatory" (by chance) materialism which entails

the negation of all teleology whether rational, moral, political, or aesthetic. I would add that such a materialism is not the materialism of a subject such as God or the proletariat but rather the materialism of a process without a subject that governs the order of its own development, without any assignable end ... And then the clinamen suddenly appears: an infinitesimal declination that one doesn't know whence, when, or how it originated. What's important is that the clinamen causes the atom to deviate the course of its fall through the void and causes an encounter with the nearest atom and from encounter to encounter ... a world is born. (Althusser quoted in Casarino 2003, 222)

Inherent in the concept of emergence, and in complexity theory (as with related philosophies such as Whitehead's Process Thought (2010)), is a certain notion that there are latent "tendencies" in the system, and that although the world is understood as always in becoming, often chaotically so, there are some patterns and orders which 
emerge. Such a notion does not necessarily assume a difference between organic and inorganic life, or between mind and matter, and thus cannot be straightforwardly described as "vitalism", but it does allude to the idea of a life force or life energy, perhaps best expressed through Bergon's (1998) idea of an élan vital. In this idea, Bergson attempts to account for evolution and processes of becoming as more lively, self-organising, and creative, than mechanistic viewpoints would have it. Inaytullah suggests that this can be a problematic idea for many because it appears to have mystical "pre-Darwinian" overtones (Inayatullah 1994, 684). On Emergence's website Fern Smith writes about the concept of emergence, explaining that

It is alchemy, the secret to re-generation. Looking for definitions of emergence often seems disappointing the explanations do not seem to match the lived experience of watching it happen. The term comes from systems theory but its modus operandi is more often deeply poetic or improbably transcendent. This word reminds me that the world is not merely material. It is a participatory universe that continually seeks to orient towards balance and health. Something is moving through it an emergent quality if we can step back, silence ourselves and be open to the possibility...

The complexity/emergent view maintains that the world is always in flux. To think of it as such is to think of time "like a kaleidoscope: the world is a matter of patterns that change, that partly repeat, but never quite repeat, that are always new and different" (Arthur quoted in Inayatullah 1994, 693). Thus, emergence is "never simply a chronological relation of before and after, but a [non-temporal] phenomenon" (Szerszynski 2017, 256).

Groves' diagram (fig 3.4) illustrates nicely how various temporalities might be visualised. It shows two versions of linear temporality, featuring abstract and emptied futures. The third illustration depicts the kind of kaleidoscopic temporality associated with emergence, resembling an organic spiral formation which, as Grosz (1999, 5) describes "builds, binds, contains, and transforms all relations, whether natural, cultural, or personal while also ensuring their dispersal, their development beyond current forms and parameters".

A useful way of distinguishing between these temporalities is with the concepts of chronos and cairós. Chronological time (chronos) refers to measurable, quantifiable, homogenous and essentially empty time a time which Casarino (following Agamben) describes as having found its "apotheosis with capitalist modernity" (Casarino 

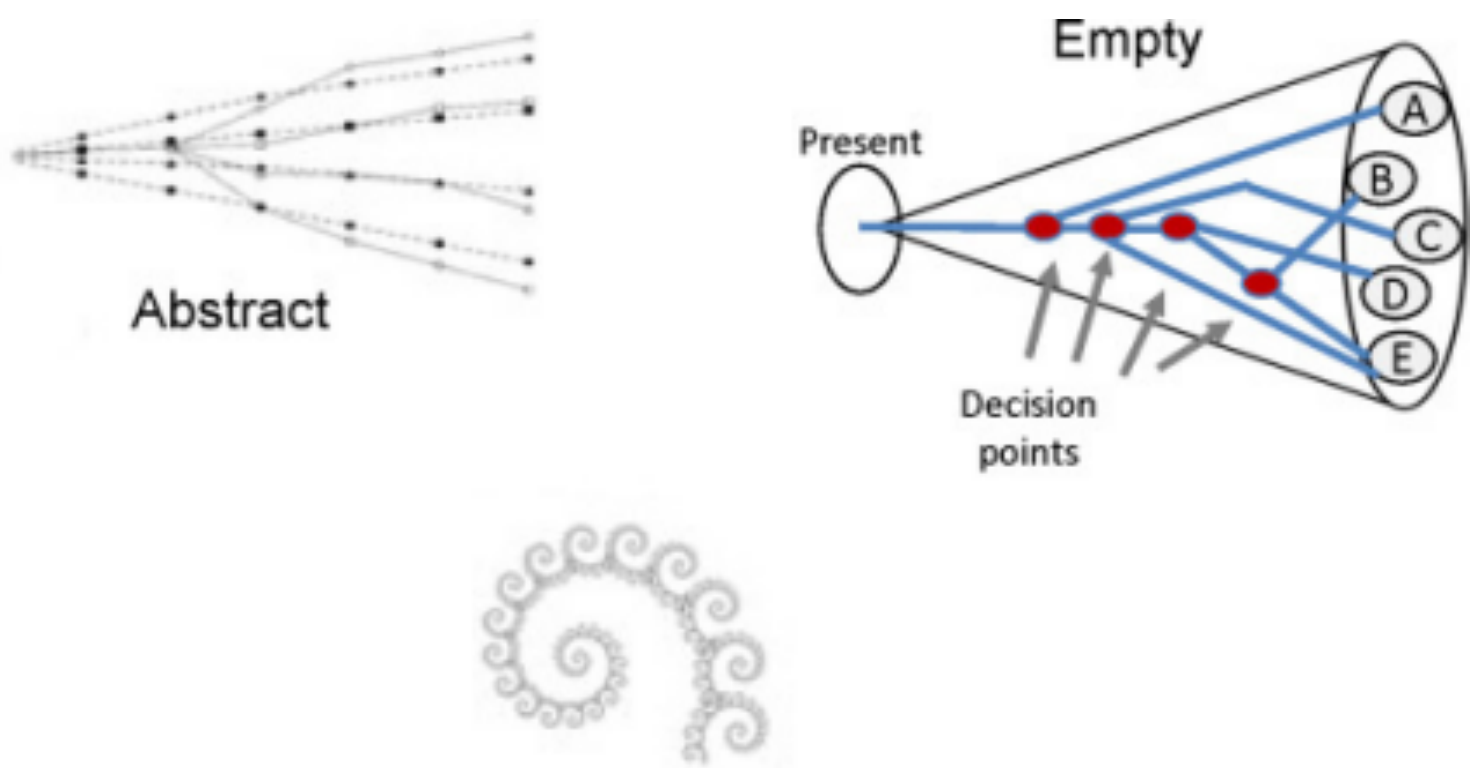

Lived

Figure 3.4: Diagrams of temporality. (Source: Groves 2016, 8)

2003, 220). This is a time in which, as Casarino puts it, we are always waiting. Against chronos, Agamben finds another kind of temporality in marginalised, forgotten cultural traditions which incorporate interruption, discontinuity, and undeferred fulfilment, especially in the concept of cairós. Cairós describes the "the abrupt and sudden conjunction where decision grasps opportunity and life is fulfilled in the moment" (Agamben 1993, 101). In other words, the shape of a cairós moment is its unanticipated intrusion on historical expectations (Falk 2016). Deleuze, too, developed a philosophy of time which resonates with this idea of cairós. He posited that time is less a flow than an interacting network of processes and elements, characterised by infinite variability, or, in other words "chaos" (see Deleuze 1994). Chaos, difference, and unpredictability are central to Emergence's emergent sense of time, and its understanding of how change happens and newness arises.

The theoretical underpinnings which I have outlined in this section highlight the rather different ontological standpoints being reproduced by the various case studies with regards to temporality. In the following section I use this basis to show how "geographies are made" (Anderson and Adey 2012, 1533) through these constructs of time, with a particular focus on how knowledge is implicated in how these temporalities are performed. 


\subsection{The role of knowledge}

The Welsh Government and ZCB both privilege knowledge in relation to the future. The Welsh government's anticipatory stance means that knowledge in the form of prediction or at least possibility and likelihood is valued. This is evident through the Future Trends reporting scheme, which forms an obligatory part of the Well-being of Future Generations Act. ZCB also takes a strongly "evidence-based" approach to the future in that it makes rigorous assessments both of the latest climate science predictions and projections and renewable energy technology, in order to be able to present a vision of the future which corresponds to both these bodies of knowledge. Not to seek some kind of knowledge, some kind of purchase on the future seems, perhaps, barbaric:

Predictable, measured, regulated transformation, change under specifiable conditions and with determinate effects, seems a readily presumed social prerequisite; upheaval, the eruption of the event, the emergence of new alignments unpredicted within old networks, threatens to reverse all gains, to position progress on the edge of the abyss, to place chaos at the heart of regulation and orderly development. (Grosz 1999, 16)

And yet in the notion of emergence there is a certain call to relinquish this reliance on "knowledge" as a bridge towards the future. Minkowski argues that futurity is essentially outside of knowledge, and that it is precisely this mystery which propels life "it makes of the future a reservoir of eternal and inexhaustible forces without which we could not continue to live" (Minkowski 1970, 81). This is not to deny the value of knowledge in general, but to say that, when it comes to the future, knowledge functions as a "mode of resistance to the play of vital forces" (Grosz 1999, 21).

This resonates with other accounts which suggest that efforts to "know" and predict the future closes down more possibilities than they create, and that a position which would allow more radical transformation is one which keeps the future fundamentally open. An attentiveness to chaos also resonates with Serre's $(1995,13)$ attempts to think outside of metaphysical categories of unity and rationality and to place contingency and "noise" as the "ground of our being" instead. It is one thing, however, to theorise about such things, and often quite another to find ways to live and enact them. As Minkowski $(1970,7)$ asks, "How do we live the future, independent of and before all knowledge?" As already discussed, knowledge, certainty, and control are often highly coveted amidst conditions of uncertainty. 
Emergence draws on the concept of synchronicity as a means by which to incorporate an understanding of complexity, uncertainty, and mystery into daily life. Synchronicity is a concept which has appeared frequently in Emergence's ethos and in my conversations with co-directors Fern and Phil. It was first introduced by Jung (1952) to denote "meaningful coincidence", that is, a situation in which acausally connected events are experienced as having a meaningful connection in the context of the subjectivity of the person having the experience. Thus, synchronicity represents a meeting of internal and external "reality", and thus alludes to the possibility of a collective unconsciousness which does not reside within individuals but is ubiquitous. Rather than trying to direct, control, or avert change, to take a sychronicity approach is to be open to the possibility of the acausality of some transformation, and to welcome the possibility that a multitude of forces or energies may be at play which we might never be consciously aware of. Significantly, according to Pauli's and Jung's (2001) development of the idea, synchronicity calls into question the conventional concept of time, for it places emphasis on meaning as an ordering factor rather than causal chains through time and space. In their exchanges with one another, Jung describes synchronicity as an "ordering system by means of which similar things coincide, without there being any apparent cause" (Jung and Pauli 2001). In other words, as Casrino (2003, 223) puts it, "things happen: all sorts of encounters take place all the time, and we have no say in the matter but as they are taking place they can be affirmed as necessary and can be actively turned into lives and worlds of our own making over and over again". On Emergence's website, Fern Smith writes about how synchronicity has informed her own ways of working, and the creation of Emergence:

I [had] consciously given myself up to "the methodology of the marvellous" [referring to Gablik's (1991) description of synchronicity] the inexplicable synchronistic process by which one attracts, as if by magnetism, the next piece of vital information. This led me to magical places, where I encountered some courageous, smart and compassionate thinkers. It also led me to Emergence and the extending circle of people I have been lucky enough to work with over the course of the project.

But, as she told me, synchronicity for her is not a passive way of working, but an active methodolgy

there is a methodology there, and, and, there is something very exact as well, with synchronicity, it's not just a kind of a vague thing. Um ... and 
it's very ... powerful ... and I think that you can work with it.

Central to Fern's interpretation of synchronicity, and indeed to the ways in which Emergence deals with the prospect of not "knowing" the future, are qualities of trust and intuition. Trust is a crucial element not only in the way in which Emergence facilitates dialogue, but also in everything from how it organises events to its philosophies for life. For example, on Emergence's website Fern describes how she went about organising one of Emergence's earliest events:

The outcome of the event was not to be predicted before the invitation was accepted. The invitation went out to those who had attended the summit locally and those who had worked on it from further afield. Trusting in Emergence uncertain outcomes, interconnectivity, developing resilience, creating a learning supportive community this is what we asked for and hoped to give our guest delegates. ${ }^{6}$

More recently, Emergence invited the peace and environmental activist Satish Kumar (a key figure for Emergence and with whom the organisation made a documentary in 2016) to speak at a peace walk in Swansea. Kumar passionately told the audience:

If you really want to experience emergence you have to just trust, trust in your heart, trust in people, trust in the universe.

Emergence draws inspiration from both non-Western and early (pre-modern) cultures in this respect, as this extract from a talk by Ian Rees (at an Emergence harvest festival in 2015) indicates:

So, when we talk about things like tipping points, or new paradigms or new ideas, we're talking I think about something really difficult ... the things we need, in my experience, is to trust and sustain our trust in what arises out of our own hearts. It's difficult really to know sometimes what arises you know we're so used to thinking about ourselves and our world in particular ways. Now what the bards would do [...] would be an embodied descent into mystery and not-knowing. And, that descent, in their time, would not just be for personal problems or personal issues, but also in response to ... the great cultural issues of their day.

To relate to the future via trust and intuition is diametrically opposed to a desire to

\footnotetext{
${ }^{6}$ Available at http://www.emergence-uk.org/wp-content/uploads/The-story-so-far.pdf. Last accessed 31st May 2017.
} 
predict and control, and indeed it can provoke deep scepticism in the context of Western knowledge traditions. I found myself initially wary of the kind of language associated with Emergence (and emergence), interpreting it as perhaps a pseudo-spiritual or "New Age" response to conditions of liquid modernity and endemic uncertainty. This remains, of course, a possibility, but on spending more time with Emergence and tracing some of the lineage of the ideas which influence it, I am more inclined to understand Emergence's emphasis on trust and intuition in the context of a serious attempt to rethink how we relate to the future, and to conceptualise how transformation happens, outside of a linear, cause-effect model. A focus on trust and intuition resonates a great deal with Bergson's and Minkowski's theories of time and becoming (see also Grosz 1999). It also resonates with Delueze's approach to non-linear time (and with Keats' negative capability concept), in which the relation to the future is "not to predict, but to be attentive to the unknown that is knocking at the door" (Rajchman 1999, 47). Only through such a relation can the present become something creative and experimental. Rajchman $(1999,46)$ writes that "it is a matter of freeing our sense of time or the future from any salvationism from judgement or judgement day and making it a matter of trust in the world." For these writers, chance is what signals the openness of the future, to the possibilities of paths of development and trajectories which are uncontained by the present "the random or the unpredictable is of the essence of a time that is not regulated by causality and determination but unfolds with its own rhythms and logic, its own enigmas and impetus" (Grosz 1999, 4). This is to insist on the fundamental openness of time to futurity, and to resist attempts to reduce time to the workings of causality. Grosz's reading of these philosophies of time also fundamentally rethinks Darwinian notions of becoming. Whereas Darwin's theory of evolution has frequently and forcefully been evoked to support and explain a linear, deterministic sense of progression, driven by natural selection (in both biological and social terms), Grosz's non-deterministic reading of Darwin's seminal works posits his theories as the enabler of conceptions of dynamic, open-ended, random, and creative becoming, owing to the centrality of chance in evolutionary processes (Grosz 1999, 2008a).

It is in this spirit that Emergence attempts to embody a trajectory without specifying a destination. Fern Smith describes it thus:

I think fundamentally, it's about holding the possibility of change, and ... making that available for people and holding you know that everything ... everything that we seem to do has that sense of ... openness and possibility to it, which makes it anathema to anything in the current 
system, you know. So ... you know fundamentally there is no outcome.

You know, always, cos Emergent systems, they're not about the outcome.

This seems to echo something that Minkowski (1970) writes:

... as soon as I think of an orientation in time, I feel myself irresistibly pushed forward and see the future open in front of me. And this fact of "being pushed" has nothing passive in it. This does not mean that exterior forces compel me to look in front of me and to progress in that direction ... It means that I tend spontaneously with all my power, with all my being, toward a future. (Quoted in Grosz, 1999, 15)

Emergence, in contrast to ZCB and the Welsh Government, is not so much interested in generating "exterior forces" (e.g. targets, goals, visions) to compel people to look ahead, as it is in providing conditions in which the possibility for change is held open, a possibility which, as Minkowski suggests, compels people towards new conditions spontaneously rather than mechanically. Emergence's desire for "knowledge" about the future is therefore markedly less than both the Welsh Government's (in terms of gathering it), and ZCB's (in terms of generating it).

\subsubsection{A brief consideration of consensus}

A final question looms over these three case studies' relationship with knowledge, and that has to do with consensual framing. One effect of a linear sense of time in which an ideal future is posited as something to aim for (a "zero carbon Britain", for example, or the seven Well-being Goals of the Welsh Government's act), is that it not only closes round one particular imaginary, thereby foreclosing others, but it also assumes a kind of consensual agreement that this is something towards which "everybody" should aim. The Welsh Government are explicit in this regard: a national consultation exercise called the Wales We Want was rolled out to inform the development of the Act, and in the short publicity video featuring protagonist, Megan, the closing words of the narrator are "This is the Wales that Megan wants. It's the Wales we all want" (emphasis in original). Further public communication about the Act states "To make sure we are all working towards the same vision, the Act puts in place seven well-being goals" $(2015,3)$. One Government official told me that the language about goals and collectivity was deliberately chosen to align with the language and framing devices used by the United Nations, both in terms of its 
Sustainable Development Goals, and its World We Want web platform, which states "we will bring the priorities of people from every corner of the world to the forefront and help build a collective vision". ${ }^{7}$ In addition, the Welsh Government's sustainable development strategy is called One Wales: One Planet, again emphasising a desire for unity and a collective cause. There are two criticisms that can be directed at this kind of consensual framing. First, as Massey (2005, 5) contends, the cosmology of "only one" narrative "obliterates the multiplicities, the contemporaneous heterogeneities of space. It reduces simultaneous coexistence to place in the historical queue." The temporal language of collective progress, then, can serve as a neocolonial mode of placing the "Other" as "behind" in time: less developed, less legitimate, and therefore somewhat erased (Klinke 2013). It is also unreflexive about the existence of multiple temporalities, multiple ways of constructing the future, and thus is unquestioning of an assumed universality of the temporality of modernity (Inayatullah 1990).

The second, related, criticism is that, in proposing "what ought to be", situations are presented with a consensual framing which mobilizes thought and action in particular ways and which are, essentially, undemocratic (Stengers 2005). ${ }^{8}$ It is a situation in which, Swyngedouw (2013b, 6) suggests, "disagreement is allowed, but only with respect to the choice of technologies, the mix of organisational fixes, the detail of the managerial adjustments, and the urgency of their timing and implementation, not with respect to the socio-political framing of present and future natures." And while Emergence's temporality seems to offer some alternatives to such technomanagerialism, with its emphasis on transformation and becoming as multiple and contingent, there is a risk, too, of this approach particularly articulations of it which contain a sense of élan vital also falling into the trap of consensual framing. Stengers $(2005,1000)$ is concerned, for example, with how models of emergence based on biological or natural science models allude to an idea of "the harmonious participation of each member in a single body" or a "good common world" an appealing idea but one which, according to Stengers, needs to be rejected as a "bad, anti-political mix of naturalism and religion" (ibid.) which imposes another form of authority ("the cosmos"). This is a crucial point because it reminds us that, even when the rhetoric embraces complexity and multiplicity, this is often a hard stance to embrace in practice. As Stengers warns, ideas about complexity can themselves arise out of a rather

\footnotetext{
${ }^{7}$ Available at http://www.beyond2015.org/world-we-want-2015-web-platform. Last accessed 31st May 2017.

${ }^{8}$ For Stengers, democracy can either refer to "the least bad way of managing the human flock, or a gamble focused on the question not of what humans are but what they might be capable of" (Stengers 2005, 1001).
} 
singular understanding of the world, one which is therefore implicitly (and perhaps unintentionally) promoted as a vision for the future.

Nonetheless, Emergence's conception of an open, fundamentally unknowable future, which emerges out of chance and novelty, provides a stark contrast with approaches, often entailed in linear conceptions of time, which seek knowledge about the future, and which set goals and visions for the future. This contrast raises an interesting question with regard to the (often great) importance placed on images and imaginations of the future as exterior forces which compel people to look ahead, a question which I now explore in greater detail in relation to the Welsh Government and ZCB, which both rely heavily on the tactic of "looking ahead".

\subsection{The question of looking ahead}

As Yusoff and Gabrys contend, "future representations [...] and their imaginative possibilities to become otherwise is part of how we might understand how possible futures are set in motion" (Yusoff and Gabrys 2011, 519), and the drive to construct positive visions of the future is increasingly being adopted by environmental movements. Zero Carbon Britain seems, particularly, to be operating in this vein. ZCB's project co-ordinator, Paul Allen, was keen to stress to me that ZCB is not intended as a "blueprint" for action, but rather as an inspiring story about the future, a way of showing people what is possible so they might get excited about it, rather than using messages of doom and gloom. In his presentations to members of the public, Allen makes an analogy with his own experience of quitting smoking it wasn't the health warnings and risks that gave him the willpower, it was meeting a friend who had quit smoking and was now a picture of health and fitness. Allen aspired to be like him, and that's what motivated him to quit smoking. And so it is, according to Allen, with climate change. He remembers in the 1950s and 60s representations of the future were incredibly exciting "flying machines and gold miniskirts" whereas now, since the 1980s or so, society's images of the future have become increasingly dire as the spectre of environmental catastrophe has increased. This leaves people with no story to aspire to, no better future to believe in. Allen gave me an example of this lack of imagination, recounting an experience he had had teaching a workshop with a group of teenagers:

... every time we look in the future we paint pictures of the future; in 
novels, films, gaming world, television, we're painting ecological collapse and dystopia, so the the majority of people are now beginning to think "that's what's going to happen". I mean I had a group in of 18 years-olds, and there was like, 50 of them, and I said ... I want you to tell me a film or a book or a novel or a TV series that you've seen that's [set] 10 or 20, 30 years in the future, where we have more or less risen to the climate challenge. And they talked in little groups and eventually somebody went "Wallace and Gromit" [laughter]. That was it! That was the best they could come up with!

Allen established ZCB to try to address this lack of imagination, and to try to challenge the paralysis of "oh dearism" (a phrase borrowed from the film maker, Adam Curtis) when it comes to thinking about the future. To accompany the technical reports of ZCB, Allen has developed what he calls the "Extraordinary story of Human Beings and Energy", which contextualises the technological changes that ZCB is envisaging within the broader sweep of human history, from hunter gatherer and agrarian societies which depended on solar, water and wind energy, to the discovery of fossil fuels and the subsequent acceleration of industry and consumer culture. Allen wants ZCB to shed light on the "next chapter" in this extraordinary story, of a shift away from fossil fuels and towards a situation where humans are once again dependent on renewable sources of energy but this time on a much more sophisticated scale. As he explained to me:

I think the aim of the project is to change how we think about rapid decarbonisation. Rather than to be seen as a sad burden that, unfortunately, because of climate change, we're gonna have to live more boring lives and we're not gonna have the exciting stuff that we've got used to ... it's much more exciting to think about co-benefits, and the increases in well-being, the better diet, the more exercise, the more time in nature and all of the positive things things that we actually want to change and it gives us a generational opportunity to change them. And focussing on a positive vision. Get people excited about it! And show that it is achievable with existing technology. To change how people think about human beings and energy. Which is why I've developed this story which sort of gives a wider perspective, over time, to that relationship.

As one video about ZCB explains, the project "helps us see what a positive future would look like". For Allen, as with the Welsh Government's emphasis on roadmaps, 


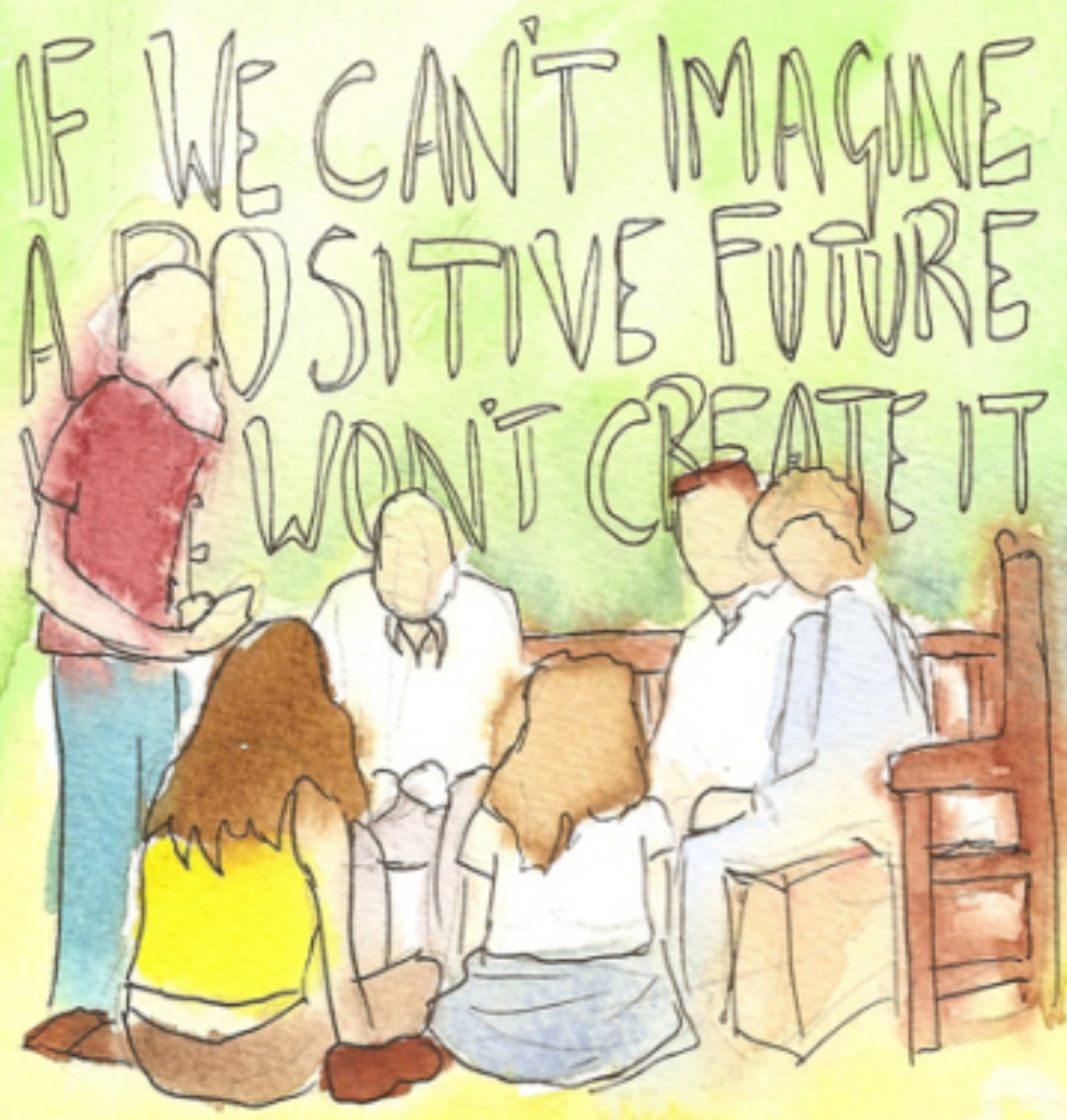

Figure 3.5: Image used in ZCB presentations and media (source: ZCB) 
milestones, and goals, there is a sense that having something to aim for is important. In ZCB's presentations to the public, an image is used which says "if we can't imagine a positive future we won't create it" (fig. 3.5), and Allen told me

it would be awful to think that we did all this big shift of infrastructure but we did it 15 years too late, so we didn't manage to stop really big serious climate change? We have to do it on time and it has to be what we call evidence-based decarbonisation, it has to be ... at the rate that will actually solve the problem? It's like you're building a bridge you have to know where you are and where you're going. (Emphasis added)

ZCB's other full-time member of staff, Philip James, added that it is important to have something to work towards:

So I think there's ... as much as we don't want to be trying to sell it and be ridiculously positive and glossing over kind of the difficulties of it ... I think you do have to kind of ... we have to try and create within society ... an idea that this can happen and needs to and then we'll work towards it.

Importantly, this kind of outlook on the future is what Groves (2016) has called both abstracted and emptied. Abstracted in the sense that the scenarios and targets draw one's gaze out to an abstract horizon, a time that is "not yet", and emptied in the sense that it assumes that the future can be whatever we make of it (as Allen says, below, "all we have to do is claim it"). Allen uses history as his guide in this sense:

That sort of thing can then trigger a radically different world in a way that perhaps ... people who grew up under apartheid could never imagine that the world ... people who grew up in slavery could never imagine a world where slaves would be liberated, or women who grew up in a very sexist society could never imagine that we'd have a woman Prime Minister! We'd have an African-American president! ... So there's a world there that perhaps we can't even imagine yet, that's very exciting and all we've got to do is claim it.

And so it is that ZCB creates a positive vision of the future which focuses on what Allen calls the "co-benefits" of decarbonisation smarter energy, improved transport systems, healthier diets, less consumption, more land for trees and wildlife, happier people and it is rigorous about showing how these changes could be brought about 
(at least in technological terms its most recent research has begun to explore social, political and economic factors in making such a transition possible, too). ZCB emphasises this positive vision by painting a contrastingly dystopian vision of the past and present, for example by highlighting current levels of energy use and waste and the insidious role of consumer advertising in driving these levels of consumption, which ultimately makes people neither rich nor happy. This, too, is characteristic of a "modern" orientation to the future, as depicted, for example, by Benjamin's "Angel of History" trope: the angel (a metaphor for modern society) is propelled into the future because it is fleeing from its horrible past. In his presentations, Allen often refers to the "fossil-fuelled dream" which promised greater and greater prosperity but which is now crumbling under the weight of evidence that this dream does not deliver, neither environmentally nor in terms of human health and well-being. The presentations feature images (fig.3.6) which depict the "normal" as outrageous, an act of "collective, suicidal insanity" (Korten 1995, 247), and which therefore establish a feeling of estrangement, of disenchantment with the status quo, making it look strange, absurd and grotesque (Fournier 2002, Soper 2008). ZCB's depictions of the future also feature photos of present-day conditions, but are selected to engender a far more optimistic outlook. In both its presentations and online videos (fig.3.7), photos and footage of renewable energy technology, weather conditions (to indicate the use of solar, wind, or hydro energies), and happy-looking people, combined with an energetic, up-beat sound-track, and narration from on-screen interviews with ZCB staff, create a sense of energy and hope for the future.

In the Welsh Government's recent publicity, notably for the Well-being and Future Generations Act (2015) and the Environment (Wales) Act (2016), one can detect a similar spirit of optimism and aspirations for an improved future (as enshrined in the Well-being Goals). However, there are some important differences with the ZCB approach in terms of how the future is represented.

Like ZCB, the Welsh Government has employed what is perhaps best described as a decidedly "cheerful" visual approach to representations of the future. Unlike ZCB though, the Welsh Government videos and summary documents utilise cartoon graphics with bold, primary or pastel colours, simple symbols and stylised representations of people and places. The videos feature happy, smiling (cartoon) people, a backdrop of lush green landscapes and blue skies, and a soundtrack of birdsong (fig.3.8). The films pan along a left to right trajectory (akin to a platform computer game), thus linking them to the linear narrative outlined above. They are narrated by a female voice in a light-hearted, optimistic tone, without complicated language. The 


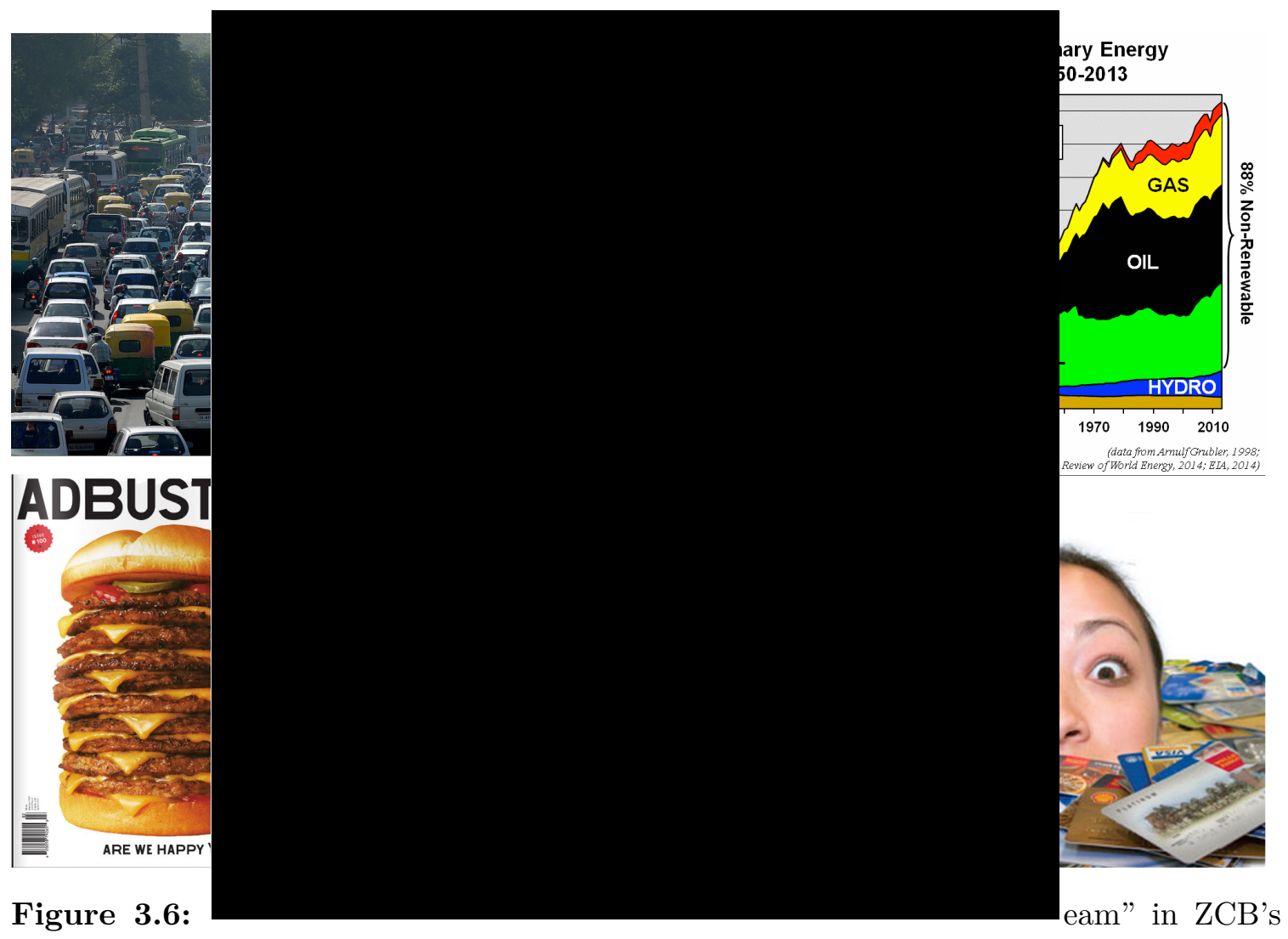

presentations. (Sources: Google Images except where specified)

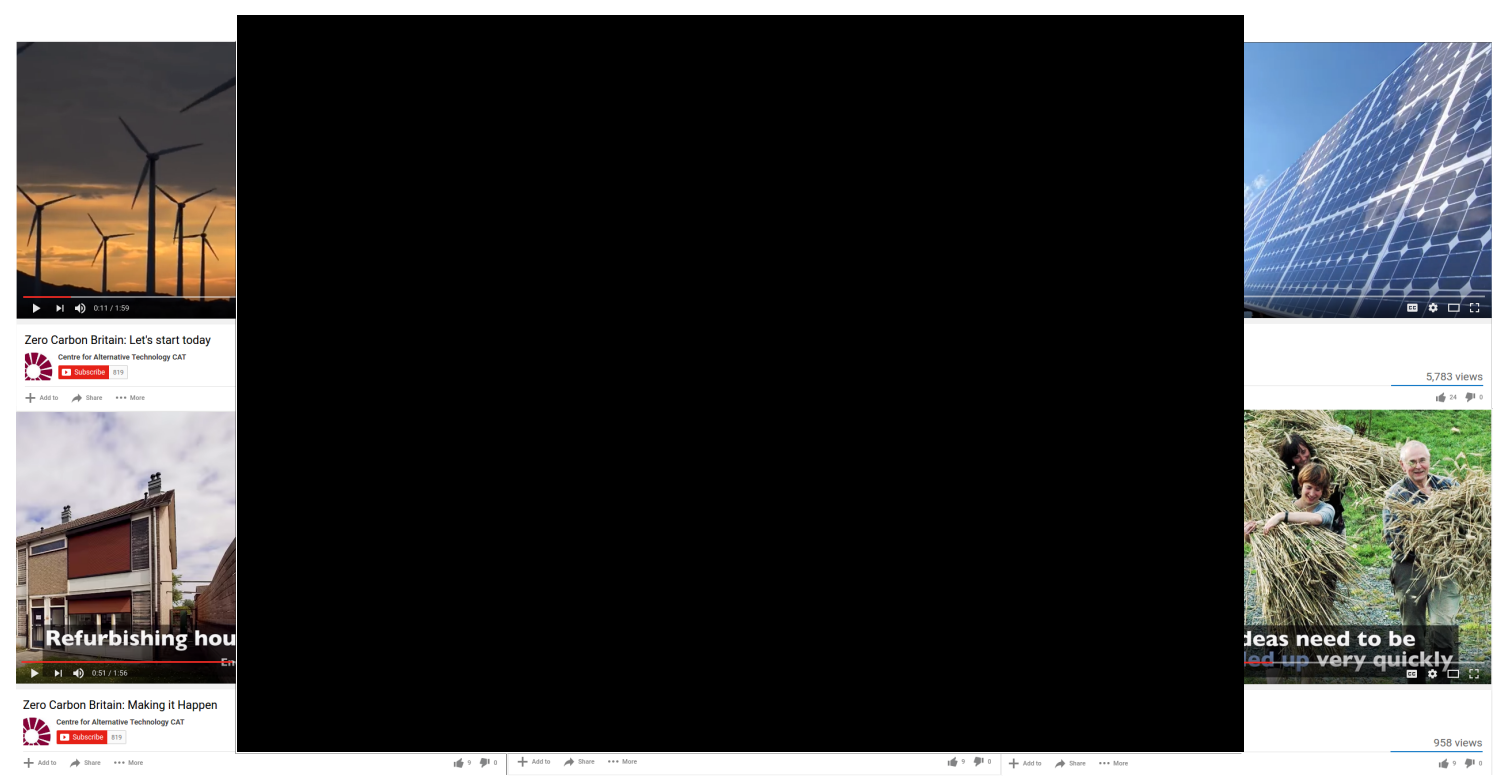

Figure 3.7: Stills from ZCB films "Let's Start Today" (2014) (top row), and "Making it Happen" (2017) (bottom row) 


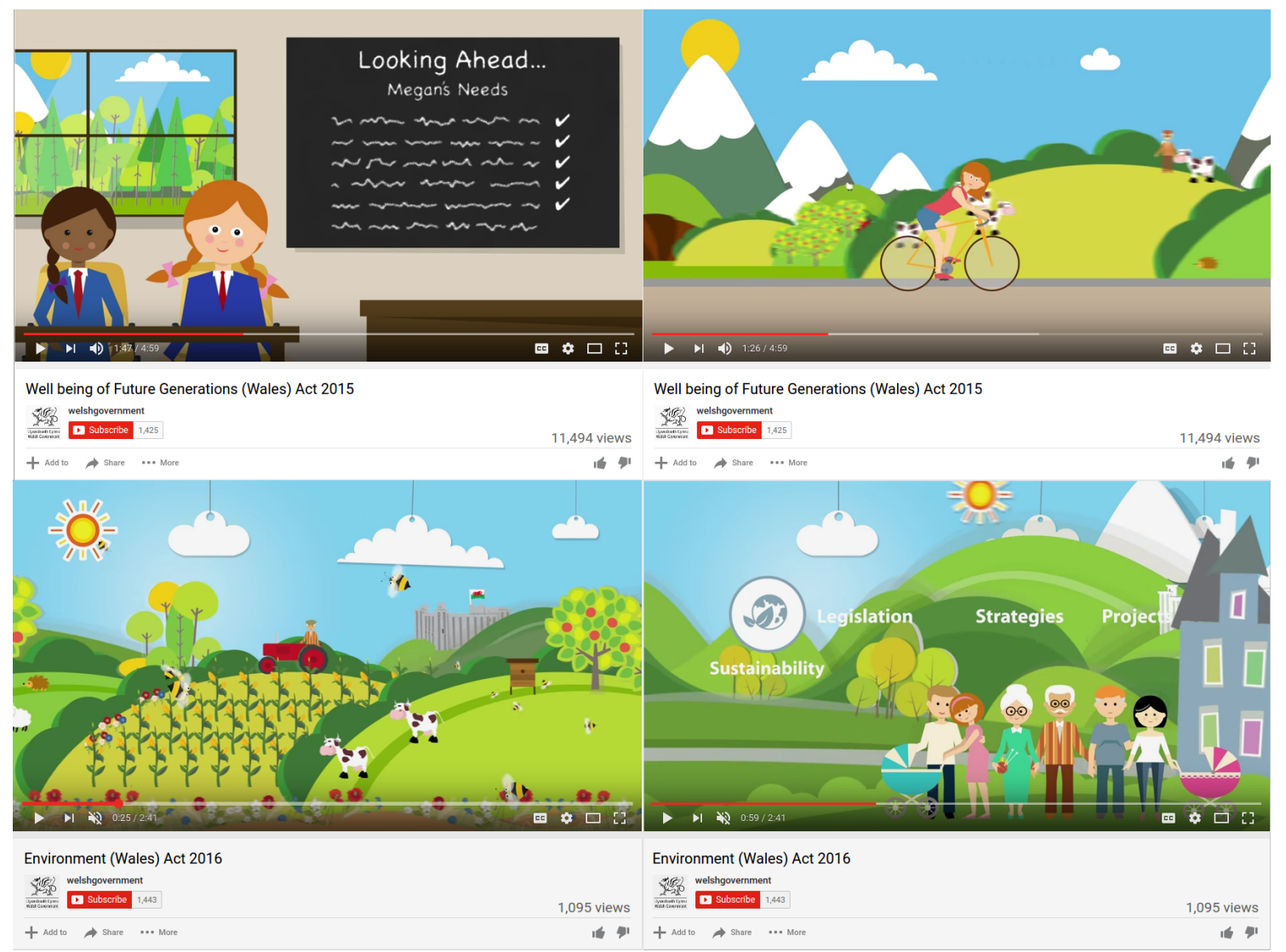

Figure 3.8: Stills from videos introducing the Well-being of Future Generations Act (top row), and the Environment (Wales) Act (bottom row)

WFGA video features a single protagonist, "Megan", and follows the course of her life through a trajectory of birth, school, college, career, family, death, highlighting the challenges she may face in meeting her needs along the way challenges which the Act is designed to address (such as poverty, climate change, health, and inequality). Notwithstanding an obvious need and desire to make publications accessible to a wide spectrum of people, it is worth, I think, exploring the possible implications of these aesthetic choices with regard to how they may shape imaginative capacities for transformation.

That the Welsh Government paints a decidedly rosy picture of the future is not especially unusual. As already mentioned, positive, hopeful stances towards the future are deemed by many as an essential motivating force for social transformation, a tactic which ZCB and many-other future-focussed organisations seem to be employing. However, the Welsh Government's decision to use simplified, cartoon images means that often the seriousness or scale of the challenge is somewhat glossed over. Climate change, amongst other socioecological concerns, is presented as a mere problem 
to be dealt with along the (seemingly inevitable?) route towards a happy future. In contrast to $\mathrm{ZCB}$, which presents its own (rigorously researched) scenarios in the context of the latest climate science (and matches its efforts accordingly), the Welsh Government proposes to deal with climate change in vague terms. One section of the WFGA film shows a smiling Megan sitting at school with a checklist under the title "Looking Ahead: Megan's Needs" written on the blackboard. The narrator says:

So, over her life, Megan has many needs. The good news is that these needs can be met. However, there are a few challenges in the way. Such as: Poverty; an increasingly global economy; an ageing population; poor health; and the inequality of health between people; climate change, and pressures on our natural resources; and rising demand for quality public services

And while this sounds all very straightforward, it fails to acknowledge that the Welsh Government's own targets on climate change (3\% reduction per year), as mentioned, are nowhere near what has been deemed necessary if extremely dangerous changes in climate are to be averted (Anderson and Bows 2008). Similarly, little if anything is said about how socioecological problems are already affecting society rather, they are presented as threats in the future. Again, the contrast here with ZCB is evident, for although they both depict a bright future, ZCB does not tend to gloss over a view of some aspects of the present as "grotesque". The Welsh Government's approach might therefore be understand in terms of what Hirt (1996) calls a "conspiracy of optimism" a situation in which the severity of the problem(s) are masked by whoever is doing the representing. Latour (2015) finds this to be a widespread problem, and contends that never before in history has there been such a mismatch between the requirements of time and space and the utopian "keep calm and carry on" approach coming from leaders.

If, then, organisations opt for envisaging decidedly "positive" futures rather than remaining ambiguously open to them, what might be the consequences of obscuring from view, other, darker, future possibilities? One compelling suggestion is that, contrary to an instinct to want to avoid pain or discomfort, confronting shared vulnerabilities in the face of overwhelming environmental crises is a crucial element in processes of individual and collective transformation. As Macy and Johnstone (2012) explain, the result of not suffering, of not confronting the dystopian possibilities of the current situation, is apathy (meaning, literally, a- 'without' + pathos 'suffering'). Other commentators have also noted the pitfalls of only focussing on the good. Feath- 
erstone and Miles $(2014,128)$ remark that "it is only when we confront the worst, and understand that the way things are now cannot continue, that we realize that we must change our situation and invent the new". Haraway (2016), too, believes that "staying with the trouble" necessarily involves the work of, as Kenney (2014, 255) puts it "inheriting violent pasts and presents in the process of building more livable worlds". Dystopian imaginaries might also be a way to empower individuals and political communities to take action to avert it (Claisse and Delvenne 2015). This proposition also resonates with the etymology of "crisis", which, as mentioned in the previous chapter, originally meant the time to make decisions (Bauman 1999). Today, the idea of "crisis" is more often associated with an atmosphere of impending disaster or catastrophe and feelings of uncertainty and helplessness one reason to want to avoid thinking about it whereas, in earlier usage, "crisis" depicted a moment of engagement and for taking sensible decisions which could secure a turn for the better (ibid.).

The Welsh Government is therefore caught in a difficult place: too much talk and imagery of apocalypse risks depoliticising issues like climate change as uncontrollable planetary inevitabilities, while too much "utopianism" prevents people from confronting pain and vulnerability, and thus creates another kind of depoliticisation: apathy. This is a tension not easily resolved, and is not the job of government alone. Nonetheless, my analysis suggests that, in the Welsh Government's approach in particular, there is a trend towards an increasingly infantalised, sugar-coated approach to socioecological transformation. The imagery and narratives employed in the recent videos create an aesthetic which belies the complexity of both the problem and the solutions. Solutions are couched in relatively familiar, safe terms "the cupcake as opposed to the messy and collapsing sponge-cake" (Whyman 2014, unpaginated). This kind of response to a crisis is reactionary rather than radical, shutting down possibilities thrown up by the crisis in favour of (re)asserting certain values, and creating an infantalised subject. Guattari ([1989] 2014, 33) vehmently opposes such a "stupefying and infantilising consensus", arguing that what is really needed in response to socioecological crises is a dissensus, and a cultivation and openness to diverse possibilities. This criticism can also be applied to ZCB, for it too depicts a rather "one size fits all" image of the future although it is careful to point out that its work is not intended as a blueprint but as a conversation-starter.

There is one more important difference between ZCB's and the Welsh Government's projections of the future. The Welsh Government's decision to use cartoons and playful graphics rather than photos of actual people or places (as ZCB have done) 
raises some interesting questions about how imaginaries and relations to the future are constructed. It is possible to understand why cartoons might have been chosen as "neutral", easy-to-reproduce graphic representations which help to build a particular image and "brand" for the Welsh Government's sustainability projects. However, I suggest that using cartoons rather than photos of actual people and places does two, related, things in terms of future imaginaries: first, and related to a linear perspective on time, it implies that the future is somewhat detached from the here and now, and that the "imagined future is a different world, inhabited not only by different technologies but inhabited by different people, too" (Nordmann 2014, 89). Second, this sense of "otherness" means that the future is essentially unrecognisable in the context of the present there is little to suggest that things already happening in society are related to possible futures, or that problems like climate change are having or will have an effect on current environments and communities. It therefore reinforces an imaginary in which the future is abstracted, always somewhere ahead or "not-yet", rather than a lived future which is inextricably part of the present (Groves 2016). Chakrabarty $(2009,197)$ describes how "the current crisis can precipitate a sense of the present that disconnects the future from the past by putting such a future beyond the grasp of historical sensibility". The Welsh Government's future is, in some respects, a "world without us" (Weisman 2008), in that images of presentday people and places are substituted by idealised cartoon forms. Adam and Groves (2007) describe how contextual, embedded and embodied futures-in-the-making can be "airbrushed from the picture, traversed and negated" (Adam and Groves 2007, 14). The danger is that this kind of artistic licence glosses over the real difficulties of transformative action (Harvey 2000), and therefore that images "do not identify agencies and processes of change. The result is that utopia moves further into the realms of fantasy" (Levitas 1993, 265). This "abstract" utopia (as opposed to "concrete" utopia which recognises the possibility of a real, possible, and transformed future) is something that Levitas, following Bloch, recognises as a distinctive feature of postmodern utopianism. Although this might have the advantage of liberating the imagination from the constraint of what it is possible to imagine is possible, it has the disadvantage of severing utopia from recognisable processes of social change (Levitas 1993), and this can be equally as depoliticising as a future imaginary which is laden with apocalyptic imagery. 


\subsection{Concluding thoughts and some tensions ex- plored}

If the notion of the Anthropocene signals, as Delanty and and Mota (2017) propose, a new cultural model which invokes new conceptions of time, agency, knowledge and governance, then the examples explored in this chapter offer some insights into how such models and ways of coping with the future are currently taking shape. There is a complex interplay of forces that shape futures-in-the-making, and in this chapter I have only visited a few. Nonetheless, the themes of resilience, uncertainty, linear conceptions of time, and emergence are suggestive of particular "ecologies of ideas" (Guattari [1989] 2014) and modes of approach which resonate in the context of socioecological transformation more widely. These themes also present some deep contradictions and tensions which are worth drawing out in a little more detail here.

In both the Welsh Government's and ZCB's case, there is a privileging of knowledge in relation to the future, both in terms of predicting future trends (in the case of the former), or of depicting what is technically possible (in the case of the latter). This seems to be fundamentally at odds with the kind of lived, emergent future advocated by Emergence, a position which says that in order for radical "newness" to emerge, then the future, essentially, needs to remain unthought, for to "know the future is to deny it as future, to place it as a given, as past" (Grosz 1999, 6). It is Emergence's commitment to this idea which means that it doesn't set out to describe particular goals, aims, or timescales, but instead proposes to "live the future now". 9 There is also some agreement in the literature that attempts to represent the future close down possibilities and narrow the imaginative space to play and experiment (e.g. Braun 2014, Massey 2005). Massey $(2005,11)$ says "only if we conceive of the future as open can we seriously accept or engage in any genuine notion of politics. Only if the future is open is there any ground for a politics which can make a difference." But how can this be reconciled with a simultaneous argument that visions of the future are important and that narrative accounts of how to get from the past to the future via the present are central to people's abilities to organise, co-operate, and create? The paradox is that any attempt to "make solid" plans for sustainability, to set out a vision and to enshrine it in legislation as the Welsh Government have done, cannot help but to close down other possibilities, other futures, and other imaginations, and evoke a somewhat linear, chronological imaginary of time. What's

\footnotetext{
${ }^{9}$ Available at http://www.emergence-uk.org/\#the-emergence-vision. Last accessed 1 June 2017.
} 
more, Negri (in Casarino 2003) takes the view that utopianism (which he identifies with Benjamin's concept Jetztzeit ${ }^{10}$ ) is in fact the motor of "progress" (progress being a watchword of capitalism), and thus represents an all-too-fleeting possibility, soon to be "retranslated into quantified and measured time, and hence flattened back into the relentless march of progress precisely because it was only a flash" (Casarino 2003, 227). Thus, utopianism is eminently co-optable by capital. Taken a step further, the kind of utopianism-as-blueprint approach has long been subject to criticism for its tendency towards totalitarianism (see, for example, Jameson 1996 and Harvey 2000).

And yet to go to the other extreme of not generating concrete images of the future, of insisting on the radical openness of the future, there is another dilemma, in that such an approach cannot account for the necessity of some forms of closure (around particular policies or course of action, for example) unless the desired state is a "romanticised and perpetually unfilled longing and desire" (Harvey 2000, 183). In addition, there is the potential for radically open futures (such as Emergence's) to create a vacuum. As Grosz $(1999,17)$ asks, "if the revolution can carry no guarantee that it will improve the current situation or provide something preferable to what exists now, what makes it a sought-for ideal? What prevents it from blurring into fascism or conservatism?" Similarly, the "open and multiple" futures approach could also be read simply as a reflection of the kind of fragmentary, noncommittal conditions of postmodernity (see also Clarke 2011), rather than a radical attempt to transform those conditions (e.g. Levitas's “ambiguous utopia" (1993)). What's more, how is such radical openness, and an aversion to planning or predicting or setting targets, tenable on the scale of modern nation states? The short answer is that it probably is not, because the aspirations of "nation-becomings" (the promise of life, status, and longevity for its citizens) are very distinct from the becomings of emergent and contingent life-in-general (Grosz 1999).

Emergence's lived philosophy on time and becoming, however, gives me pause for thought. In particular, it casts some doubt on the assumed role of images of the future, of the importance of the "not yet" and of horizons of hope and aspiration in socioecological transformation. There can be little doubt that representations of the future are folded into the present in dynamic ways which influence all manner of aspects of daily life (Anderson and Adey 2012). And yet, does trying to imagine and envision desired futures perpetuate a model of linear time which abstracts and

\footnotetext{
${ }^{10}$ In Benjamin's Theses on the Concept of History ([1942] 2009), Jetztzeit is a notion of time which challenges chronological progression, and sees time as "here-and-now", poised with revolutionary energy, ready to take a "tiger's leap" into the future.
} 
empties the future, continually postponing it and fuelling some of the ideological assumptions about growth and progress which are so environmentally deleterious? What might this striving and yearning do to our abilities to transform the present? These are some of the questions which arise from a consideration of alternative temporalities, such as Emergence's "living the future now". Such understandings of time and becoming can be traced to a variety of contexts, from Western scientific understandings of complexity theory and emergence, to the philosophies of time posited by Bergson, Deleuze, Minkowski, Grosz and others. Versions of the idea of "lived futures" can also be found in several Eastern traditions, notably Buddhism, which emphasises the importance on the present moment, and of slowing down and becoming more aware of one's immediately lived experiences. With notable similarities to the aforementioned theories and philosophies of time and becoming, the Buddhist tradition teaches that the mind is not separate from matter, and therefore that patterns of thought flow out into the environment. Incessant brain-chatter the racing back and forth between past and future which generates feelings of dissatisfaction, craving, longing, and anxiety is, according to this line of thought, a form of selfharm which also ripples out into the environment (see Gaard 2014). The alternative to "racing ahead", then, as Stengers (2005, 994) echoing Levitas albeit in a less mechanistic way suggests, is "to 'slow down' reasoning and create an opportunity to arouse a slightly different awareness of the problems and situations mobilizing us", ${ }^{11}$ in a similar way that some Eastern philosophies suggest that attempts to know and understand the future moves one further away from understanding reality, rather than closer to it (see also Inayatullah 1990, 118). This is not to disregard an ethical responsibility towards those lives that are yet to come, but rather to understand the future (and an ethical responsibility towards it) as fundamentally lived, that is, not a transcendental construction in spiritual or material space (Inayatullah 1990) where the time for action is forever postponed (Falk 2016), but understood as arising from the social fabric of everyday life.

Levitas's ideas about utopianism are useful for thinking about these tensions. Levitas maintains that utopianism is important for social transformation, but in her "Utopia as Method" thesis (2013) and work leading up to up to it, she shows that utopias (historically) have not always been located in the future, but in a spatial "elsewhere" (Levitas 1993, 259). In fact, as Levitas points out, it is only with the advent of "ideas of progress and the belief in some degree of human control over social organisation that the location of utopia in the future, as a point to which society

\footnotetext{
${ }^{11}$ The 13th Century Persian poet Rumi's famous lines "Sit, be still, and listen; For you are drunk, and we are at the edge of the roof" seem to sum this sentiment up perfectly.
} 
either inexorably tends or can be pushed by human action, becomes a possibility" (ibid.). The possibility of removing the temporal dimension and yet retaining some of the socio-psychological benefits (hope, enthusiasm, will, for example) associated with utopianism (Latour 2008; Beck 2010), is therefore intriguing, and resonates somewhat with a position (like Emergences's) which avoids speculating about or positing future visions, but nevertheless is still "utopian" in that it is underpinned by a desire for change. Levitas's concept of utopia as method seems to chime with the idea of lived futures, for it posits the here-and-now as the space to be transformed and attended to, and, crucially, recasts the future as a problem of conceptualising agency in the present. In an earlier essay, Levitas $(1993,265)$ writes "the solution [to the severing of utopia from processes of social change] is not to call for more and better utopias, more and better images and maps of possible futures. These will follow when we have better analyses of the present which identify possible points of intervention, paths and agents of change. The fault lies not in our stars, but in ourselves."

My own experiences with the Welsh Government's, ZCB's, and Emergence's temporalities therefore leave me feeling somewhat conflicted. Although there are plenty of criticisms which could be made about the consensual framing and linear imaginaries of ZCB and the Welsh Government, I am simultaneously impressed and excited by them in many ways. ZCB's scenario, in particular, gives me a sense of (apparently justified) hope that transformation is possible. My own experience thus puts me somewhat in agreement with the argument that images of the future are important motivational forces. And yet my involvement with Emergence has led me to consider other possibilities, for it has indeed aroused a slightly different awareness of the situation in me. This is an awareness of a much deeper sense of transformation, one which goes beyond superficial (but nonetheless essential) changes to energy infrastructure and political institutions (for example), and which calls into question the very ways in which time and transformation are thought about and enacted. In particular, it is Emergence's focus on what is unknown and unknowable which has, I think, much to offer. In Stengers's alternative definition of "cosmos", the word does not refer to a particular thing or unified, harmonious universe, but to "the unknown constituted by these multiple, divergent worlds and to the articulation of which they could eventually be capable" (Stengers 2005, 995). This is not to deny knowledge, but instead to demand that "we slow down, that we don't consider ourselves authorized to believe we possess the meaning of what we know" (ibid.). If each case study might be thought of as a different character, each vying for my imagination in terms of narratives, visions, aspirations and proclamations about the future, then more often than not I 
have found that Emergence, with its ideas about time, uncertainty, and becoming, is to quote Stengers $(2005,994)$ again the one that "slows the others down, [that] resists the consensual way in which emergencies mobilize thought or action. This is not because the presentation would be false or because emergencies are believed to be lies, but because "there is something else more important". Perhaps it is this space of slowing, resisting, of acknowledging the unknown, which holds out possibility for the most radical forms of socioecological transformations, for it makes room for the bubbling up of dissensus and novelty, and helps one to gain a glimpse at how particular futures and temporalities, which have emerged as seemingly universal, might not be so universal after all. It is therefore a means by which we might see beyond the particular versions of temporality and futurity we have constructed for ourselves (or have been constructed for us). As Inayatullah (1990, 134) says, paraphrasing Heidegger, "we hope for a future time, yet we exist in quantified, commodified time; we hope for a world outside of who we are, yet our awareness only encounters what it has admitted beforehand as an object possible for it". To slow down and to become attentive to the possibility of cairós, emergence, unpredictability, is thus to begin to "change time", and it is this, as Agamben (1993) ambitiously suggests, that is the true task of genuine revolution.

This does, however, leave me wondering what role narratives and stories about and for the future play in this context. Can stories of socioecological transformation be non-temporal? Do they need destinations, a beginning, middle, and end? Are stories inherently chronological, or can they incorporate something of cairós, too? And, relatedly, is imagination of the future the same as imagination for the future (the former being a more instrumental use of imagination which attempts to know the future, the latter being an imaginative capacity to live emergently). Many argue for the need for new temporalities if we are to escape the chronological, quantifying time of capital and modernism and thus find more socioecologically sustainable ways of living (see Inayatullah 1990 and Casarino 2003, discussing Agamben, Negri, and Marx). But whereas those such as Agamben and Grosz look towards the revolutionary potential of the now (the realisation that change does not necessarily depend upon waiting), and of the idea of lived futures-in-becoming, contingent upon chance and intuition (a "time without time"), others suggest a new sense of time which is stretched, encompassing a longer temporal vision and sense of deep time, which nurtures an ethics of care and responsibility towards future generations (e.g Inayatullah 1990, Adam and Groves 2011). Inevitably, this chapter opens up more questions than it answers. This is particularly true with regard to an almost unspoken tension be- 
tween how to balance, on one hand, an undeniable need to take action on (especially) climate change, an issue which is virtually impossible to disentangle from ideas of time and futurity, and, on the other hand, how not to whittle down possibilities, or to set motions of action and thought on particularly narrow trajectories which remove from present and future generations the opportunities to make their own decisions, and their own worlds. Although this is a difficult tension to resolve, especially in the context of bureaucratic and political constraints, perhaps it is useful to think of the approaches to time outlined in this chapter not as opposites but as parallel. That is, they need not be mutually exclusive, but can coexist. Indeed such an approach might enable the most flexible and fruitful forms of socioecological transformation, albeit only if these varying forms of temporalities can coexist in slightly better balance (i.e. with more credence given to the emergent and the unknown) than is usual in most "mainstream" approaches to environmental problems. 
CHAPTER 3. 


\section{Chapter 4}

\section{Cultures of nature: imagining and performing more-than-human worlds}

Western civilization countries have this expression "going back to nature", which I've never understood. I'm rather keen on going forwards to nature! (Bjork, 2016) ${ }^{1}$

\subsection{Introduction}

In the previous chapter I situated socioecological transformation as a temporal issue, and I explored the ways in which this temporality manifests itself in some of the case studies. The chapter concluded that a linear perspective on time and futurity, where the future is imagined as something "out there" and "yet-to-come" (Groves 2007 , 1), can have the effect of severing the future from the present and the past, creating subjects as observers "outside" of time. In other words, transformation imaginaries which play out along axes of time might fail to take into account more immediate, complex, and non-linear forms of temporality which are commonplace in everyday experience. While calls for more contextualised, participatory, lived, futures-in-the-making (e.g. Adam and Groves 2007) go some way to addressing this

\footnotetext{
${ }^{1}$ Crack Magazine interview, With all the Earth's Electricity, by Jazz Monroe, Issue 68, September 2016
} 
problem of abstraction by understanding the present rather than the future as the focus of transformation, there was a significant omission from my discussion of this issue: space. For all the temporality of talk about more social and ecologically just futures, it is easy to forget that these issues are intractably spatial, too, concerning relations and interdependencies between people, places, and species. Any responses to current crises and the creation of alternative ways of organising need, therefore, to accommodate the simultaneity and heterogeneity of lives lived spatially, as well as temporally. The question of socioecological transformation might also be posed as the question "how are we going to live together?" (Massey 2013a, unpaginated), which is as much a question of simultaneity (space) as it is about succession (time). By levering imaginaries into narratives of time, perhaps there is a risk of overlooking the transformational potential that alternative conceptions of space can bring.

On this, Massey's thoughts on (a radical re-imagining of) space are illuminating: “... temporality has been extolled as the vital dimension of life, of existence itself. The argument here is that space is equally lively and equally challenging, [...] far from it being dead and fixed" (Massey 2005, 14). The kind of spatial imagination that Massey proposes (one that sees space not as an empty stage but as full of, and constituted by, a multitude of dynamic relations) brings with it many of the qualities one might consider central to socioecological transformations. First, it is to be able to imagine difference and simultaneity of "stories-so-far" lived out across the world: to "take on board coeval multiplicities" of space, and to deal with "constitutive complexity" (Massey 2005, 8) rather than simplified, linear stories of development. Second, Massey suggests that a spatial imaginary which understands lives as simultaneously lived with billions of other interdependent and interrelated lives all sharing the same planet may ignite an ethics of care and responsibility towards these relations (Massey 2005), an ethics which tends to be elusive in future imaginaries where the object of concern is always "out there" and "not-yet". And third, space (Massey suggests) is an utterly political realm, whereas "the future", imagined as a temporal realm, is increasingly seen to be depoliticised through narrowed-down imaginaries of either apocalyptic dystopias or sugar-coated utopias. As Massey says, "thinking the spatial in a particular way can shake up the manner in which certain political questions are formulated, can contribute to political arguments already under way, and most deeply can be an essential element in the imaginative structure which enables in the first place an opening up to the very sphere of the political" (Massey 2005, 9). Perhaps a focus on space, with all its complexities, differences, and radical potentialities, 
can help puncture the feeling that "there is no alternative" (Harvey 2000). ${ }^{2}$

In this chapter my aim is therefore to engage with spatial imaginaries via an exploration of how relations between humans and more-than-human others are imagined, constructed and performed by two case studies, Cae Tan and the Welsh Government. The qualities of such relations are central to how space is constructed, and to the ways in which humans currently co-habit with other life (human and more-thanhuman) and, crucially, how it is envisaged it could be otherwise. I focus on two very different organisations here because they both appear to develop ideas about interconnectedness, but in rather different ways, and thus they highlight how a current vogue for relationality (in policy as well as in academia) needs to be carefully examined in terms of how such rhetoric actually acts in the world (Mansfield and Doyle 2017). In addition, the two case studies highlight just how heterogeneous imaginaries of socioecological relations can be, and shows how those that are constructed at the more visible, official, level of policy, can be challenged and enacted otherwise in other areas of life. Cae Tan and the Welsh Government have an intriguing relationship in this respect, because, although the Welsh Government's performance of human environment relations seems to contradict Cae Tan's approach, the Welsh Government has supported Cae Tan through various funding initiatives, and thus there is a relationship between the two that hints at a degree of understanding and mutuality. Before turning to the case studies, I first review some of the context for thinking about the social construction of nature, particularly in light of the concepts of imagination and performance.

\subsubsection{Imagining "nature"}

Few commentaries on the social construction of "nature" fail to reference to Williams' inclusion of the word in his volume Keywords: A vocabulary of culture and society, in which he states that "nature is perhaps the most complex word in the [English] language" $(1976,219)$. Yet despite the nebulous, abstract nature of the word "nature", it has enormous social power, holding sway over ideas about anything from

\footnotetext{
${ }^{2}$ It should be noted that my use of the terms space/time and spatiality/temporality are themselves not straightforward concepts, and point to particular epistemologies. In this chapter, I refer to them as relatively distinct concepts (as is common in geographical literature), but this is a position which is challenged in some areas of physics, for example, in the concept of space-time (as in Einstein's theory of Relativity), where space and time are fused together. The more dynamic, political understanding of socially-constructed space that I draw on here does, however, try to avoid an overtly dichotomous dualism in which space and time are understood as opposites (for more on this matter, see Massey $1992 ; 1999)$.
} 
wilderness (Cronon 1996), morality and ethics (an idea about what is "natural" gets applied to diverse topics, from sexuality to diet), to green consumerism. To take a social-constructionist perspective is therefore to understand "nature" not as a concrete "given", something out there, waiting to be experienced or interacted with (Castree 2013a) but instead as a concept which is constructed and mediated via a whole host of meaning-making practices. From this perspective we can begin to see why understandings and imaginations of what nature "is" have important implications for the ways in which humans interact with and behave in the world. A perceived separation of human culture from "wild" nature first emerged, it has been suggested, from the Christian doctrine of domination (White 1967), and was subsequently perpetuated via secular, scientific practices of seventeenth and eighteenth century Enlightenment (Harvey 1996, Anderson 2014b). The separation and domination trope is commonly understood to be one of if not the most problematic and destructive ideas in history, in terms of how relations of exploitation and despoliation, not only between (some) humans and other species but also between humans, are legitimised and maintained. Feminist and ecofeminist theory makes explicit how the subjugation of "nature" (which arises from a masculine idea of human mastery over nature), is an idea which is inseparable from the subjection of women and other marginalised groups (e.g. Merchant 1980, Gaard 1993, Plumwood 1993). It is with these and many other violences and injustices in mind that Moore $(2015,4)$ writes that the nature culture binary "drips with blood".

Over the past few decades, critical engagement with the idea of "nature" has helped to dismantle this binary (Descola 2013), and within academia many arguments are made for doing away with the idea of "nature" altogether (e.g. Morton 2007). Such arguments are also finding their way into public discourse (for example, McKibben's (1989) The End of Nature, and a recent article about nature in the Anthropocene in the Guardian newspaper, featuring the philosopher Timothy Morton ${ }^{3}$ ). Nonetheless, what to do with the knowledge that the nature culture binary is an "absurd" one remains a vexing problem (Moore 2015), and in "nature's" place, there has been a proliferation of different ways of imagining and constructing relationships between humans and the more-than-human world, so-called "social natures" (see Jones 2009 for an overview). Consequently, political struggles are no longer necessarily about "naturalness", but about "what should be done, by whom, to bring about which social natures, and to the benefit of whom (human and nonhuman)" (Mansfield et al. 2015, 284). The outcomes of these struggles are central to the shaping of socioecological

\footnotetext{
${ }^{3}$ Available at https://www. theguardian.com/world/2017/jun/15/timothy-mortonanthropocene-philosopher. Last accessed 24 June 2017
} 
futures; as Jones (2009, 295) suggests, "the stakes are high".

The battle for competing visions of social natures feels or at least is commonly depicted in the literature as being particularly tense at the moment. There are a number of reasons for this, which I will briefly outline here. The first can be traced to a growing interest in notions of non-duality and relationality, including movements in posthumanism, assemblage theory, actor-network theory, and materialism. These literatures put forward ontologies which, in various ways, seek to dismantle a view of humans as transcendent subjects in an otherwise inert, objective world, and instead put forward alternative views of how we might relate to ourselves, to one another, and to the more-than-human world. Posthumanism, for example, "rejects the notion of the separability of humanity from the non-human world ... Rather, it seeks to recover the complex ways in which humans are entangled with non-humans" (Franklin 2007, 1) and Ginn $(2017,1)$ adds that the term "emphasizes the different ways humans are continually produced through material forces, discursive regimes, and through nonhuman agencies. One of posthumanism's key aims is to dissolve binary distinctions that characterise humanism, most notably culture/nature and self/world". Posthumanism offers terms such as "more-than-human" (Whatmore 2006), "multinatural" (Bingham and Hinchliffe 2008), "hybrids" and "cyborgs" (e.g. Haraway 2008) to try to move beyond humanist traditions and towards a recognition of how "human" bodies and activities are in fact constituted of a multitude of more-than-human assemblages with more-than-human others: the boundary around "human" is in fact endlessly porous. Relatedly, relational-materialist ontologies (see Anderson and Harrison 2010), which can include the prefix "vital", "immanent" or "new", also maintain an understanding of the "human" which does not stand apart from its interaction with an array of "microbes, tools, technologies and other organisms that operate above, below and beside the body, shaping its genetic forms and its subjective and cognitive powers" (Braun 2013, 2), and as such seeks to recognise the active participation of nonhuman forces and agencies in events (e.g. Bennett 2009). It is worth noting here that while posthumanist and materialist movements are largely directed at challenging the centrality of the "human" (and the idea of a separate "nature") in Western philosophy (Ginn 2017), these literatures themselves have been subject to criticism for risking reproducing colonial ways of knowing (with often very Eurocentric scholarship) by subordinating and/or instrumentalising non-Western, indigenous ontologies (see Sundberg 2014).

Nonetheless, non-dualist ontologies of matter and the de-centering of human subjectivity increasingly move towards an imagination of "nature" which is vastly different 
to a dualistic, Cartesian one. This brings me to a second factor contributing to the current zeitgeist centred on social natures: the rise of the so-called "new sciences". As introduced in the previous chapter, complexity theory and related fields such as quantum theory, which have risen to prominence in the last few decades, specifically challenge an idea of nature as fixed, stable, equilibrium-seeking, and linear, and instead conceptualise the world in terms of dynamism, non-linearity, and nonequilibrium, with a tendency for small perturbations to create dramatic changes (for example, notions of "tipping points" and "regime shifts") (e.g. Bohm 1951, Prigogine and Stengers 1984). Significantly, many of these ideas have been imported from the physical sciences to the social sciences (e.g. Barad 2007, Haven and Khrennikov 2013, O'Brien 2016), thus contributing to a sense in which humans are increasingly considered as elements in and of a much wider meshwork of agencies which behave in a complex manner (Dittmer 2014), and, indeed, are increasingly being used to understand the world more generally (Urry 2005a). Complexity has therefore come to characterise a particular "structure of feeling" across a whole range of fields (Urry 2005b, 1), perhaps most popularly exemplified in the idea of "Earth as Gaia" (that is, as a living organism) (Lovelock 1995) and the growing field of Earth System Science (e.g. Steffen et al. 2006).

While it is generally accepted within critical geography that these emerging nondualist ontologies offer radical potential for thinking and being otherwise when it comes to imaging humans and "nature" as always interconnected (Braun 2013), these ideas and practices are themselves also unleashing new forms of politics, particularly, as Mansfield and Doyle $(2017,22)$ contend, with regard to "efforts to engineer a range of new natures, including bodies, ecosystems, and the earth system writ large". Nowhere is this more apparent, perhaps, than in the idea of the Anthropocene, as discussed in the introductory chapter. For Mentz, there is a need for "dynamic narratives about our relation to the biosphere" (Mentz 2012, 587), while Braun (2015b) suggests that the ontological and epistemological questions raised by the Anthropocene facilitate and necessitate an "experimental turn" with regards to how socioecological relations are now assembled (see also Lorimer 2012). It is in a similar vein that Szerszynski et al. (2003) claims that new "nature-cultures" are being co-produced through improvised, staged, ritual, and everyday activities. These kinds of "performances" are how presence the ways in which the world is felt, sensed, experienced, and related to is created (Schieffelin 1998). The following section reviews some of the ways in which human environment relations can be thought about in terms of performance, as well as how representational practices also play a role in bringing 
into being particular kinds of socioecological imaginaries.

\subsubsection{Performing socio-ecologies}

Ways of knowing are kinds of doing. (Stirling 2016)

Following the kinds of shifts in understandings of human environment relations outlined above, Szerszynski et al. (2003) contend that relations between humans and the more-than-human world are not static structures but activities which themselves produce meaning, affect, and knowledges. This is reflected in the emergence of nonrepresentational theory (NRT) in recent decades, which although building on the same ontological foundations of social constructivism (that nothing is "given") seeks to move beyond a narrow focus on representation and cognition, because this in itself reproduces a binary: between "the world" on one hand and its "meanings" on the other (Anderson and Harrison 2010). The notion of performance helps to illuminate some of the ways in which nature society relations are felt and sensed in subtle ways which are "not well served by noun-dominated languages" (Szerszynski et al. 2003, 1). This is not to say that language itself is not important language can be just as performative as it is representative; language does something as well as signals something (Castree 2014c; see also Austin 1975). Performance is a useful concept to think with in relation to the case studies in this chapter because the term encompasses a range of practices which can be understood as bringing into existence particular socioecological assemblages and knowledges. These include practices of repetition, iterative processes, and "following scripts" (e.g. Goffman 1959, Schechner 1988, Butler 1990) (indeed, things have to be continually performed to exist at all (e.g. Butler 1997)), as well as more ephemeral, unpredictable, and improvised performances which also constitute, and are integral to, socioecological relations (Crouch 2003, Kershaw 2012). None of this is to say, however, that representation does not matter indeed, when it comes to "nature", representational practices can be understood as part and parcel of creating particular performances, atmospheres, and structures of feeling. As Castree $(2013 \mathrm{a}, 37)$ reminds us, "we rely on myriad others to form our own ideas, hopes, opinions, values and worries about everything", and he insists that, even while "what matters" in our lives is not reducible to the creation, circulation and effects of various representations, representations can nonetheless shape feelings, dispositions, and habits of action in the broadest sense. Representations are not necessarily the antithesis of emotion, affect, embodiment, and practice, and nor are they synonymous 
with speech and sight only, and to draw a false dualism between representational and non-representational approaches, "seems to require a new version of an old division between thought and action ... mind and body" (Nash 2000, 657). Representation takes place in, and is made possible by, a backdrop of non-representational activity (Anderson and Harrison 2010). With this in mind, I have approached the themes in this chapter, broadly speaking, via a notion of "affective atmospheres". Anderson (2009) uses the concept of "affective atmosphere" to refer to the background "hum" of conscious and unconscious factors which make up the ongoing construction of our socioecological realities (see also Thrift 2004). These are the kinds of ambiences, moods, feelings, tones (perhaps best thought of as "collective affects") which are central to our experiences of the world but which we may be aware of only subliminally or tangentially. I use both performance and representational lenses to help me do this. I draw on my own experience, observations, and conversations to try to capture something of the intensities, feelings, and affects involved (while also bearing in mind that in trying to "capture" more ephemeral aspects, I necessarily end up representing them), while also engaging some representational approaches to help elucidate practices of meaning-making which reside in discourses, texts, images, and practices. This is particularly the case in my analysis of the Welsh Government (below), because the nature of the research has been necessarily textual rather than ethnographic. My attention to representation is not with a view to rendering framed, fixed, and inert "all that ought to be most lively" (Lorimer 2005, 86), but rather is to try to remain alert to the ways in which acts of representation both create and reproduce particular atmospheres that weigh upon ${ }^{4}$ and emanate from society. These atmospheres matter because, as already mentioned, the shifting figure of the environment underpins all kinds of assumptions, practices, beliefs and forms of governance (Pugh 2014). How we describe the world reflects, at some level, how we experience it, and vice versa. Correspondingly, the ways in which narratives and experimentation regarding socioecological relations evolve are of great importance for the ways in which socioecological crises are interpreted and responded to (Gillard et al. 2016). In the following sections I turn my attention to Cae Tan and then to the Welsh Government (occasionally using text boxes to highlight contributions from other case studies), in order to develop a detailed account of how these organisations are narrating, performing, and experimenting with social natures.

\footnotetext{
${ }^{4}$ Karl Marx asks "the atmosphere in which we live, weighs upon every one with a 20,000-pound force, but do you feel it?" (quoted in Anderson 2009, 77).
} 


\subsection{Cae Tan}

Cae Tan is the largest Community Supported Agriculture (CSA) scheme in Wales, having been established in 2014 as a collaboration between Swansea-based Gower Power, a community co-operative which leases the land for the farm, and Tom O'Kane, the grower ${ }^{5}$ who set up and now runs Cae Tan with the support of one other paid member of staff, Lizzie Walshaw, and a group of regular and not-so-regular volunteers. Cae Tan is part of a growing movement in "alternative agriculture", including organic, ecoagriculture, and permaculture, which are generating new ideas and practices for growing food (Ingram 2007). The CSA model is an aspect of Cae Tan's approach which I will return to later. First, however, I focus on another distinctive feature of Cae Tan's ideas about, and performance of, human-environment relations, which is its biodynamic method of growing food (Cae Tan is the only CSA in Wales which practises biodynamics). Biodynamics is a method of growing food which emerged from a series of lectures by the scientist and philosopher Rudolf Steiner, delivered to a small group of farmers in Poland in the 1920s, and which now has a following of practitioners across the world, with over 5000 biodynamic farms registered across 50 countries worldwide (98 biodynamic farms are registered in Great Britain, covering a total area of $4331 \mathrm{ha}^{6}$ ). Steiner, who referred to his work as "spiritual science", was influenced by the German writer, Goethe, who, amongst many other things, theorised about the centrality of personal meaning and intuition in humans' relationship with, and perception of, the world (Paull 2011). Steiner's philosophy of anthroposophy, from which biodynamics stems, postulated a spirituality which was understood "not on a religious or individualistic basis, but as a set of predictable, natural forces that guide and shape physical and emotional formation" (Ingram 2007, 307). Accordingly, Steiner's conception of biodynamic agriculture recognised the farm as "a living organism" (Paull 2011, 32), and the method emphasises the importance of "cosmic, ethereal, and astral forces, which shape animal and plant growth and development" (Ingram 2007, 307). Biodynamics, through a range of on-site practices, aims to concentrate or build these forces. In his initial lectures, Steiner ([1924]1993) suggested that "we must approach everything in farming with the conviction that in order for the whole thing to work, we need to pour life and also astrality into everything around us." In what follows I draw on my experience as both a member of Cae Tan (I receive a weekly share of Cae Tan's vegetables) and as

\footnotetext{
${ }^{5}$ At Cae Tan, the term "grower" is used rather than "farmer", a decision which, straight away, indicates a desire to distinguish themselves from more conventional forms of agriculture.

${ }^{6}$ Available at http://www.demeter.net/statistics. Last accessed 17 June 2017.
} 


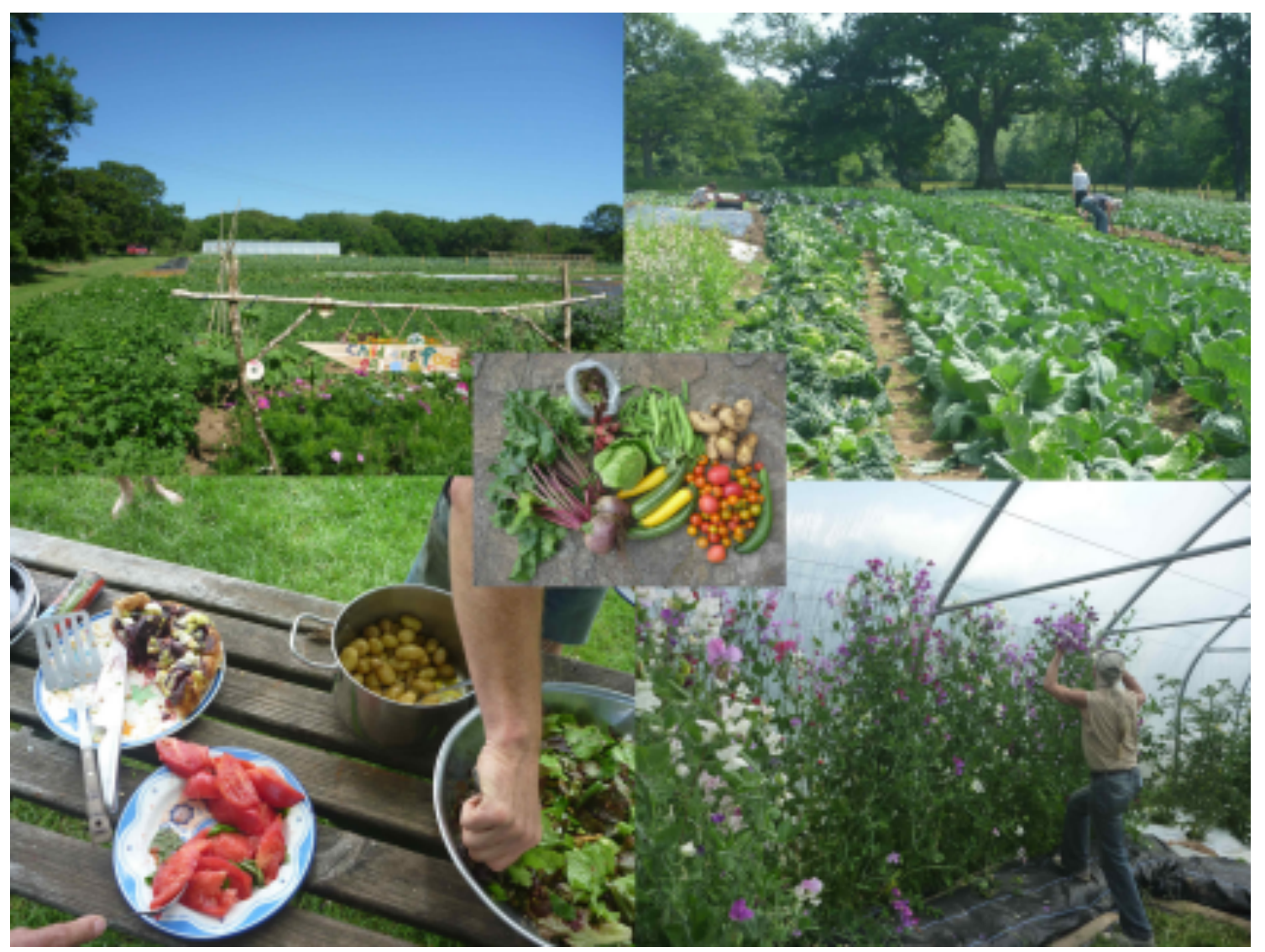

Figure 4.1: Snapshots of life at Cae Tan, clockwise from top left: The Cae Tan field; harvesting vegetables; lunch for volunteers, prepared using vegetables from the field; harvesting sweet peas as a colourful, scented addition to the members' vegetable boxes. Centre image: a summer crop of vegetables.

a volunteer at the field during 2015/2016 (fig.4.1) to explore some dimensions of the biodynamic method with regards to how particular atmospheres, ideas, and relations with and about the more-than-human world are constructed.

\subsubsection{Matter and energy}

Biodynamics, in common with other alternative agriculture (for example, organic farming), is a method that pays close attention to soil life and fertility. It is worth, therefore, starting "from the ground up", as it were. In biodynamics, soil is not viewed as inert matter, a mere substrate for growing, but rather as a complex ecosystem, imbued with energy and life. Seen through the lens of performance, a biodynamic approach, and the narratives which accompany it, "enrols" more-than-human actors such as fungi, bacteria, and trace elements in soil and elevates the importance of these networks of actors over the ability of humans to affect soil fertility via industrial- 
agricultural methods such as the Haber-Bosch system of artificial nitrogen fixation which is now widespread in modern agriculture. This is not an especially "new" attitude towards soil, although up until very recently it has been a rather marginalised one $^{7}$ : in 1947 Howard $(1947,212)$ wrote "it must never be forgotten that living organisms and not human beings are the agents which make compost", and elsewhere Coleman $(1989,99)$ writes "even if I thought I knew everything I would rather let it be done for me by the real experts . . . activities of bacteria, fungi, dilute soil acids, chemical reactions, rhizosphere effects, and countless others we are unaware of". Biodynamics is distinctive because it performs this attentiveness to the "micro-world" of the soil via very particular material practices. Following biodynamic guidelines, the Cae Tan growers make specific herbal preparations at certain times of the year and administer them to the fields, crops, and compost piles to promote fertility. Fertility is understood in both a material (microbial) sense as well as what some might call an "ethereal" sense, as this quote from Tom O'Kane, describing the contents of one of the biodynamic herbal preparations (a mixture of cow manure and herbs, packed into a cow's horn), demonstrates:

it's manure from a cow that's fertile, that's got a calf, so already [the preparation has] got that fertility influence from the cow, and the cow's basically got this extremely long intestine that's got like loads of microbes in, so you get all the fertility from the intestines ...

This perceived "chain" of fertility extends from the soil, to the nutrient content of the plants growing in it, and so to the health of the people eating them. Tom explained to me how the biodynamic process is about "connecting everything up", noting that when the soil isn't fertile, the plants can't access a full range of nutrients and consequently there is a general "dullness" of life which extends to humans when they eat nutrient-poor plants. Such a sentiment hints at what Deleuze calls a "vagabond materiality" , that is, a sensibility to the mutability of matter, its instability and activeness (Bennett 2007), and the "conductivity" of material qualities through a series of transformations. It highlights, too, the ways in which edible materials, in particular, are active "inducer-producers", co-constituting the very molecules of our bodies, thus blurring the boundary between "inside" and "outside" (Bennett 2007, 134). As Bennett (ibid. 145) contends "food as a self-altering, dissipative materiality is also

\footnotetext{
${ }^{7}$ Indeed, it seems to add a post-human/materialist quality to Marx's idea of "metabolic rift", which saw rapidly depleting soil fertility as evidence of rupture in the nutrient cycling between "nature" and capitalist human societies, thereby hindering sustainability (see Foster 1999).

${ }^{8}$ Gilles Deleuze, 'Metal, metallurgy, music, Husserl, Simondon', delivered in Vincennes, 27 February 1979, available at www. webdeleuze.com. Last accessed 19 October 2017.
} 
a player. It enters into what we become".

Ideas about energy and forces are also central in biodynamics, as a means by which these fertile qualities and materialities are shared and distributed. I attended one of Cae Tan's biodynamic preparations in July 2015, in which one of the cow manure/herb mixtures was administered to the fields, and Tom described how he envisaged the work that energy does:

if you imagine the earth's energy pushing out in the summer and everything grows, like all these tomatoes are shooting up, but then in the winter, all the focus is back in the earth. So it sort of goes backwards $\ldots$ it goes in on itself. So, you're kind of trapping that inward energy ... everything that's happening here at the moment is actually happening under the soil in the winter, all this sort of busy, life force. So you're kind of trapping that, in this cow manure in the cow horn ...

An attentiveness to energy also includes the "energies" of daily weather, seasonal conditions, and planetary motions. Tom went on to explain that

the fertility [preparation] for today, the cow manure one, is all about building that downward, fertile energy in the soil. So you've got, like [today] the moon is descending, [and] you [spray it] after three o'clock when $[. .$.$] the day is coming to an end, um .. ideally the moon in its phase,$ will be closing, um ... but it depends whether you can get it all right or not and also, ideally you'd have a day like today, not necessarily raining, [but] it should just be like a heavy, muggy, overcast sort of downwardpushing day.

This description of the "downward-pushing", heavy, energy of the atmospheric conditions, and the connection Tom makes with this kind of energy and the fertility of the soil and the timing of the preparation, is particularly interesting. It resonates with what Jackson and Fannin (2011) have called "aerography" the experience of air as simultaneously meteorological (what Tom is describing in the above quote might also be described as high pressure, high humidity conditions by a meteorologist) and affective (see also McCormack 2008). That is, the atmosphere is imbued with a sense of feeling and meaning, as something which surrounds us a "weather world", where there is an "involvement of the land and air" (Adey 2015, 57). What's more, to wait for the "right" conditions is to heighten one's awareness that the air, the winds, and humidity, are to a large extent beyond human control (Rose 2014). Rose (2014, 218), 
drawing on Levinas, says "the wind, for example, appears from nowhere. Its presence forces itself upon us and we are at its mercy. We mourn the loss of warm sun on our skin or ache for the appearance of a cooling breeze. We want these things but have no recourse or claim on their coming and going". For some, it has been the taking-for-granted of the elements and in particular of the atmosphere which has resulted in so many of today's environmental woes. Abram $(2014,301)$, for example, asks "what is climate change if not a consequence of failing to respect or even to notice the elemental medium in which we are immersed? Is not global warming, or global weirding, a simple consequence of taking the air for granted?" In addition, it is possible to read this attentiveness to the connections between soil, atmosphere, and planetary motions as an attentiveness to depth, and to interconnections in three dimensions rather merely across Earth's surface a "willingness to think through the compositional depths and dynamics of the planet" (Clark 2017, 216). There is something of an "airy poetics" (see Choy 2011, Engelmann 2015b) to the way in which Tom describes the centrality of atmosphere to the performance of biodynamics, a way of developing an elemental imaginary and a practice of "affinitive listening" (Adey 2015, 62) that is, an awareness of the simultaneous material, affective and aesthetic impressions of air which might simultaneously stimulate an awareness in us of how other beings and things also attune to air (Engelmann 2015b). While emerging work on airy imaginaries, atmospheres and poetics is helping to develop vocabularies and concepts to grasp at air's meaningfulness in the making of human and more-than-human geographies, much of this work is based on artistic and/or scientific and technological engagements that are overtly about air (for example, meteorological balloons, flights, and artistic depictions of breath (see McCormack 2008, Engelmann 2015b, 2015a). My analysis of Cae Tan helps to extend this body of work by exploring the kinds of airy poetics which abound in the more obscure, perhaps unexpected, areas of life, and how these, too, are (always and already) shaping socioecological imaginaries.

Tom's description of atmospheric conditions also hints at a sensitivity to the affective atmospheres of non-humans and to subtle flows of energies that are understood intuitively rather than empirically. As $\operatorname{Choy}(2011,157)$ writes, "[a]ir muddies the distinction between subjects and environments, and between subjects. This thickness and porosity rendered by air is part of what makes the air and the airborne such deeply felt elements. In this respect, practising biodynamics represents something of "a gathering or folding of landscape and subjectivity" (Adey 2015, 57): soil, air, and non-human beings are understood and performed as possessing varying qualities and forms of consciousness, and possessing their own particular atmospheres (see 
Lorimer et al. 2017). For example, Tom contrasts the cow horn preparation with another one which uses a stag's bladder:

the cow is this earthy heavy thing, it's always got its head down it's always eating, and they say that the cow's consciousness is not in its head, it's in its belly, it's got like ... four stomachs ... so they say the cow's consciousness is more in its belly that in its head ... so a cow like processes the land, and its consciousness is totally processed through its gut? And the stag, is completely the opposite, it's like the most alert creature, it's all up there [head], and it's like totally awake and really skittish, and it's getting that energy, and its bladder is totally related to it's the first thing that sort of releases in fear, so it's getting that total awareness and brightness, and combining it with the total opposite of the cow.

The various herbs and plants (yarrow, chamomile, nettle, oak, dandelion and valerian) are also used for their various medicinal and energetic properties (yarrow, for example, if associated with qualities of strength and protection), which are thought then to be passed to the soil, to the plants, and so to whoever eats them. The use of animal parts in biodynamic preparations is one of the more controversial aspects of biodynamics ${ }^{9}$, and in some ways appears to be at odds with a methodology which otherwise claims to care for and nurture biodiversity (Paull 2011). This highlights a tension between the "flat ontologies" (DeLanda 2013) entailed by some materialist approaches (i.e. the claim that all entities are on an equal ontological footing, with no one thing possessing greater "dignity" than other objects), and a question of ethics ${ }^{10}$. To recognise that all objects are equally "real", lively, and possessing of agency is not necessarily to deny that some objects (in particular, humans) exercise power and influence in the context of some assemblages. This poses the question of how human perpetration of non-human suffering and death ought to be conceptualised within a supposedly flat ontological landscape. There are several possible responses to this question. On one hand, some strands of ecofeminism, particularly vegetarian/vegan-feminist approaches (see Gaard 2002, Adams 2015), would find the wilful exploitation of any animal incompatible with a claim that humans are not superior to other beings.

\footnotetext{
${ }^{9}$ For example, see https://stopsteinerinstroud.com/category/biodynamics-2/. Accessed 20 June 2017.

${ }^{10}$ At this point it is worth mentioning that Cae Tan by no means claims to have a "vegan" ethicit keeps bees and chickens on its land, and many members, including the grower, Tom, have a "sheep club" in the neighbouring field; a flock which they take it in turns to tend to and from which they get a home-grown supply of lamb.
} 
To perpetuate harm on other species seems to engage in a peculiar double-standard whereby justice only applies to human life, therefore reinforcing the very dualism (between human and non-human) which new materialisms claim to overcome. On the other hand, others (for example, Derrida (1991) and Haraway (2008)) claim that the idea that we might somehow escape harming other beings or avoid unequal relations is a fantasy and that it is more appropriate to think of entities as partners in an "ongoing, unavoidable dance of relating" (Armbruster 2011, 44). Haraway's (2008, 80 ) belief is that "there is no way of living that is not also a way of someone, not just something, else dying differentially", and her response is therefore not to side-step the issue by pretending that entirely equitable relations are possible, but instead to become more sensitive to these complex interrelations. Haraway wants us to develop our abilities to respond to and respect the suffering of more-than-human others, and therefore to take harm seriously in order to keep "inequality from becoming commonsensical or taken as obviously okay" (Haraway 2008, 77). It is important to note in this context that any extension of the notion of "ethics" to non-human others doesn't necessarily entail a posthuman stance, because it still treats humans as the seat of value in evaluating the world. While this doesn't entirely resolve ${ }^{11}$ whether or not the use of animal parts in biodynamic preparations is at odds with an "ethical" approach (see Bryant 2011 and related blog posts ${ }^{12}$ ) I think it does suggest that this practice doesn't necessarily preclude Cae Tan from engendering a sense of awareness of and sensitivity towards non-human others (plants, animals elements and so on), and thus an ability to exercise power in a more responsive and to respectful way. There is no easy, fair, or "right" solution, but perhaps there are ways to find better, more careful ways of negotiating the "dance" (Greenhough and Roe 2010, Armbruster 2011).

I will leave this issue to one side because it will take me down a path which is not entirely relevant to this chapter, but it is worth bearing in mind as an interesting question mark in the context of the discussion of gratitude and interdependence that follows.

\footnotetext{
${ }^{11}$ In fact, an important aspect of both Derrida's and Haraway's arguments is that these ethical tensions can never be fully "resolved", and that all we can try to do is to become more aware of the entanglements, interdependencies and contradictions of our life styles.

${ }^{12}$ See https://larvalsubjects.wordpress.com/2012/06/01/flat-ontologyflat-ethics/ and https://larvalsubjects.wordpress.com/2012/06/09/the-question-of-flat-ethics/. Accessed 3 July 2017.
} 


\subsubsection{Ritual and spirituality}

The biodynamic preparations are mixed and administered to the land via particular rituals (at specific times each year, according to planetary alignments and weather conditions) which help to perform an understanding of the more-than-human world as energetic, interconnected, and conscious. In July 2015, I joined a small group of Cae Tan members who had come, mostly out of curiosity (Tom had put out an invite to Cae Tan members via email), to help in the preparation of the above-mentioned cowmanure and herb mixture for application to the Cae Tan field. It was a pleasant but humid summer's day, and hadn't long stopped raining, and the cow-manure mixture (which had been packed into a cow's horn buried underground for several months over the winter and spring) had been dug up, ready for use. Tom showed us the moist, crumbly, dark mixture after he had decanted it into an old mug, talking about the fertility and vitality which was locked into the mixture (fig.4.2). We collected water from the nearby stream (Tom noted that rain water would have been "even better" another indication of the attentiveness to elemental properties entailed in this method, but also of the flexibility and "improvisation" in the ways in which it is performed, seeing as no rain water had been collected), and poured it out into a copper barrel (copper, Tom explained, is also thought to be an ideal material for this purpose). The cow-manure mixture was first mixed with a little of the stream water and warmed over a stove, and then added to the water in the barrel. The mixture then needed to be stirred, by hand, continuously for an hour. Tom explained that the vortex created by the stirring motion was intended to mimic the movement of the planets. We sat around chatting, asking Tom about the process and discussing the various properties of particular plants and herbs, while taking it in turns to plunge an arm into the water and stir vigorously for as long as our muscles would allow us, before handing over to the next person being careful not to let the vortex subside. Tom explained why the method of stirring was important:

you mix it in a spiral, so you cause like a vortex you build up the speed of the water, and in about 1520 seconds, you can see right to the bottom of the barrel? And then basically as you see to the bottom, then you turn the other way and it just causes chaos and everything breaks. And every time you cause chaos, it kind of imprints ... well it imprints different things, it imprints, like, the influence of that day, and what's going on, so all the atmospheric conditions ... you're kind of trapping them and printing them on the liquid that you're gonna spray on the land, and also 
your intentions as a group of people or an individual, sort of just naturally goes into it

Contrary to what one might expect for a ritual, the atmosphere was not sombre or regimented sometimes our conversation drifted to entirely unrelated topics, and jokes were also shared. At one point during the stirring process, Ant (Gower Power founder, land owner, and Cae Tan member), laughed that his "left back-hand" stirring motion was "rubbish" and that he feared if there was a patch on the field that doesn't grow properly this season it will be his fault. Finally, the mixture was poured out into plastic trays, one for each of us to carry under one arm as we walked across the field, throwing the water from the tray with the other hand so that it would fall in an arc across the soil and crops. This motion (akin to that of scattering seeds) is also intended to mimic the motion of the planets.

Regardless of the efficacy of such rituals in terms of crop health (more on this later), what struck me was how it performed particular ideas about the more-than-human world, and socioecological relations. For example, having joined the activity with no prior experience of biodynamics, I was somewhat surprised to find myself feeling a personal responsibility towards the preparation and the soil: like Ant and his worries about his "rubbish left back-hand", as I stirred the water I wondered if my technique, or even my thoughts, might affect the quality of the preparation. I wondered what $I$ was imprinting on the water. The stirring of the water, the talk of intentions and atmospheres and energies, therefore alerted me to the possibility of ethereal connections, feedback and relations between humans and environments which are not entertained in more mechanistic approaches to agriculture. Moreover, the time spent carrying out the ritual was itself an immersion in the elements the water on my skin, the grass I sat on, the air I breathed and this is a space in which new understandings or attunements can arise, a chance "for the mind to mull over what floats by on the affective tide, or to swerve from its course as momentum decreases. Undoubtedly these are openings for learning" (Gibson-Graham and Roelvink 2010, 322). The biodynamic rituals might therefore be understood as part of producing particular "bodily states and attitudes towards ecological systems" (Smith 2011, 62). Leopold's (1949) notion of land ethics postulates that for a transformation in human relations with the land, there is not so much a need for intellectual persuasion but for a different affective orientation to the land it's not something that can necessarily be taught but must be felt. For Leopold, an ecological ethics is a matter of bodily impulse. The ritual of stirring water with one's arm is certainly a direct way to engage the body, from the sensation of the water to aching arm muscles, as is the subsequent 


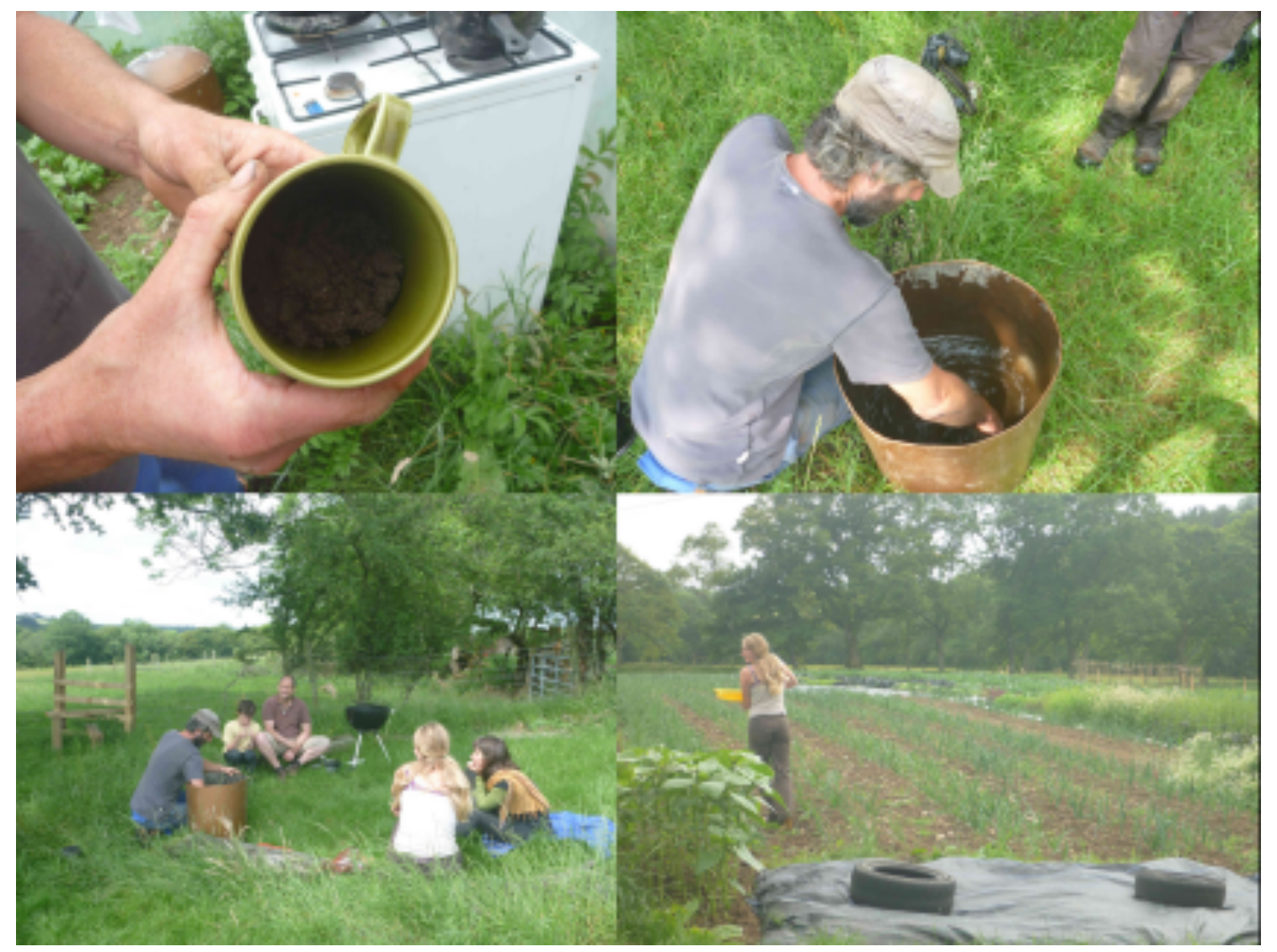

Figure 4.2: Biodynamic preparation clockwise from top left: the cow manure/herb mixture; Tom stirring the water to create a vortex in the copper barrel; Lizzie walks barefoot, using her hands to throw the the mixture across the crops to her right; the hour-long stirring process is shared amongst us while we chat.

sprinkling of the mixture across the fields by hand. That all the participants chose to walk barefoot across the furrows during this process is perhaps testament to the importance of bodily sensations (wet grass and warm mud under feet, for example) in performing these relations. In addition, sharing these movements and sensations with a group of people created shared experiences of landscape (Szerszynski et al. 2003). Even if my decision to take my own shoes off was less of a "bodily impulse" than a desire not be the odd one out, nonetheless, the experience alerted me to new ways of experiencing the land and a feeling of relatedness with the people I was with. The concern with planetary movement which is apparent in biodynamics also adds an additional dimension to land body relations, extending them to incorporate universal and cosmic imaginaries, too (see Last 2017).

Ritual seems an unlikely response to ecological concerns, and is often met with scepticism and wariness (Grimes 2003). And yet, as Grimes notes, through ritual it is possible to cultivate bonds be they psychological or physical or both (one could ar- 
gue these are not separable, in any case) between humans and between humans and the more-than-human world. Grimes therefore believes that ritual can be good for the planet, not because it is something that is easy to prove, but because much wellgrounded speculative theory suggest that it could be. However, Grimes $(2003,43)$ is careful to differentiate between an idea of ritualised human life being conservative, "one-dimensional, stereotyped, and inflexible", and a much more enlivened understanding of ritual. In this latter understanding, environmental rituals are a way of bringing performance, theatre, and creativity outside, instead of staged within theatre walls (Schafer 1991) (see also text box 4.2.1), a means by which participants might, as Rappaport $(1999,125)$ puts it, "enliven the order that they are performing with the energy of their own bodies, and their own voices make it articulate. They thereby establish the existence of that order in this world of matter and energy". This is reflected in a comment from the Cae Tan volunteer, Geoff:

Well ... life is not embodied, necessarily, but it can become embodied by going through a certain process ... so, it's not the other way around. I mean that's just a fact, the planet whatever life is being generated on this planet is flowing through us, we're not creating that life ... you know. So, at least a respect for nature and the nurture 'oh the sun on my face, the wind in my hair' picking berries, and all this sort of thing that is integral to being human!

For Rappaport, ritual is necessary for human survival, for it is the only way certain kinds of meaning can be expressed. To take a less anthropocentric view, however, we might understand the work of ritual in the context of "placework" (e.g. Larsen and Johnson 2016, Bawaka Country et al. 2016), a methodology whereby place and self are co-constituted. The ritual approach to biodynamic preparations I have described above may have an anthropocentric aim (to produce healthier and more abundant plants for humans to eat), but it is underpinned by an understanding that place has agency, irrespective of human presence and awareness. Acknowledging this, Larsen and Johnson (2016) suggest, leads to a different understanding of the geographical self to a more-than-human geographical self. As Geoff also gets at with his comment "we're not creating that life" (above), a ritual may be performed by humans, but humans are not considered the sole players. Rather, the humans are participating in a "collective experiment" with more-than-human others, and no one thing is necessarily in control (Latour 2011,3). Rituals enable an emotional, embodied understanding of this as well as an intellectual one, and this, as Grimes, like Leopold, contends, is necessary for a change in "one's characteristic tilt in and 
towards the world" (Grimes 2003, 33).

In ritualising, Grimes (2003) claims, human beings discover, then embody and cultivate their world views, attitudes, and ethics. For attitudes to become definitive they must be cultivated by practice; performance is therefore an integral process in and of ecosystems (Kershaw 2012). Stengers's take on ritualising suggests somewhat in contrast to Rappaport's (1999) suggestion that rituals are declarations of certainty and order that the efficacy of ritual lies not in saying "what ought to be", or offering any prophetic revelation, but rather is

to catalyze a regime of thought and feeling that bestows the power on that around which there is gathering to become a cause for thinking. The efficacy of the ritual is therefore not the manifestation of a Goddess who might inspire the answer but that of a presence that transforms each protagonist's relations with his or her own knowledge, hopes, fears and memories, and allows the whole to generate what each one would have been unable to produce separately. (Stengers 2005, 1002)

Grimes (2003) also talks about how a resurgence of environmental rituals in recent years marks an attempt at the recovery of the sacred. In my experience, Cae Tan's approach certainly fosters a sense of sacredness, not only in its conception of the soil as a living entity, but also in terms of a sense of gratitude towards the land, which goes beyond the biodynamic rituals to the wider ways in which Cae Tan works. For example, it has revived a tradition of celebrating Harvest at the beginning of Autumn each year, and organises a party with music, dancing, games, and beer, in the near by Heritage Centre in Parkmill, Gower. Since 2015, a few members have also organised a "Wassailing"13 event each January, an ancient ritual of celebrating, singing (and drinking) to the health of the apple trees (of which there are some planted on the field adjacent to Cae Tan), to scare away "evil spirits" (through the loud banging of pots and pans, and shouting) so that a better harvest of apples might follow in the Autumn. It is important here to reiterate that none of these ways of relating to the more-than-human world entail an entirely "equitable" relationship humans are still expecting to be able to reap a harvest from the vegetables, or from the apple trees. Indeed, the phrase "above all else we wanted and still want to create a place where natural resources are cherished and valued", which was used in a Cae Tan fund-raising campaign, exemplifies a mix of stances towards the more-than-human gratitude

\footnotetext{
13“Wassail" derives from the Old English was hál, meaning "be healthy". The tradition derives from Pagan times and was particularly popular in the cider-producing regions of West of England.
} 
alongside a paternalistic language of "resources". But the expression of gratitude to plants, weathers, soil microbes (and more) for making any of this possible marks an important attitudinal shift from the construction of a nature which is seen and felt to be inferior, and there solely for human exploitation and control. The marking of the seasons, the embodied rituals, and the celebration of (rather than a taking for granted of) are ways of performing a more interdependent sense of self. The Cae Tan volunteer, Geoff, told me that the reason he got involved with Cae Tan was in part about

actually appreciating that our very lives are given to us as bounties, you know ... we dream ... the sense organs that we are observing everything through, you know ... these things are amazing gifts, you know you're provided with all that you need ... you know, it sounds like a throwback to some sort of biblical thing but it is just a fact you know, er, the planet itself is what's producing us, and every other life form on it ... it's kind of like the planet is the, er, the petri dish, if you will, that everything is growing on ... it's the petri dish ... nothing can survive [without it].

He continued

So we are a part of the process, we are not separate from it ... the idea that us human beings are somehow separated off from all this and that we can have objective opinions about it is quite frankly ludicrous. So, until people can at least accept that and that flies in the face of what a lot of people who place far too much importance on their own, shall we say intellects ...

Notions of interdependence, gratitude, humility, and co-becoming have much in common with panpsychist philosophies which are increasingly being invoked to help understand some of the spiritual dimensions of materialism (Rigby 2014). For example, Mathews finds points of confluence with aspects of Aboriginal and Daoist philosophies, particularly in a favouring of "practices of conserving and cherishing 'the given' that which already exists replenishing the sources of renewal in natural cycles so that 'production' is accomplished largely by the world itself, without our having to direct and and design the process" (Mathews 2006, 99). Similarly, Gaard (2014) finds commonalities between the new materialism and Buddhist philosophies of interdependence, something which chimes with Tom's comment about biodynamics that 
it's just reconnecting, when you go through the whole process, it's like reconnecting you with the soil, the food you're eating, all the atmospheric conditions, where the moon is, what the planets are doing, and it's just sort of ... you're kind of working with all of it, in a very sort of practical, getting-your-hands-dirty way.

Importantly, rituals such as the biodynamic one described above enact and imagine a more-than-human world within which humans participate and co-operate, rather than control. Celebrating "the given" also alludes to a rather more benevolent imagination of the world than one in which "nature" is constructed as wild and dangerous, one which must be either subdued, avoided, or out-witted. It constructs a notion of human agency which aligns "with rather than against the grain of existing conativities" (Mathews 2006, 99), and thus resonates with an idea, as championed by the Russian geographer Kropotkin, that evolution involves co-operation as well as competition. Kropotkin ([1902] 2012) challenged what he saw as a misinterpretation of Darwin's theory of evolution which privileged an idea of "nature red in tooth and claw", and instead highlighted the ways in which social and biological "mutual aid" is also essential to survival amongst all forms of life.

Participating in Cae Tan's biodynamic ritual and in the Harvest festivals and Wassailing events, I gain a sense that what is being performed is not any kind of monolithic new religion (Rigby 2014), but rather a notion of spirituality (even if that spirituality is not explicitly named) that "arises from matter's own inner principles" (Mathews 2006, 94). This spiritual materialism emerges from "a diversity of situated practices of invocation and thanksgiving appropriate to the cultural imaginary and ecosocial contexts" (Rigby 2014, 287). Such practices tend to be place-sensitive, improvisational, and participatory activities which "co-produce" nature cultures (Szerszynski et al. 2003). As I have suggested, such practices might not altogether do away with ideas about human transcendence (for the rituals can be seen in terms of manipulation of nature as well as celebrations of it), but which nonetheless greatly enhance a sense of relatedness, care, and responsibility (e.g. Bawaka Country et al. 2016). Accordingly, rituals, ceremonies, and rites can be instructive with regard to human environment relations as much as for what they instruct people not to perform as well as what to perform. 


\section{Text box 4.2.1 Women Dancing}

Another, quite different, ritual performance takes place every year on Caswell beach, not far from the Cae Tan field. Woman Dancing is an off-shoot of the Emergence project, organised informally via facebook invites shared amongst networks and friends. Women Dancing is described as a celebration of the Spring Equinox, and involves women of all ages gathering on the beach just before dawn to dance to Patti Smith's album Horses as the sun comes up, which each person listens to on their own headphones (a silent disco-esque equinox ritual!). Part of the facebook invite reads:

This is a dance of gratitude for the cycles of nature to the sun for coming up, for the spring for coming round and for the sea for coming in [...] The invitation is to dance alone or dance with others. It's not about the choreography it's about letting yourself be danced and letting your soul play. You can dance for the sea, the sand, the sky, the sun, someone you love or someone you've lost. The ritual will be the ancient rite of moving our bodies in celebration in the open air.

There is an obvious link with ecofeminist theory and praxis here, given the connection promoted between women and nature. One could criticise this for being a rather essentialist, identity-politics driven version of ecofeminism given its conflation of women and nature (Merchant 1980). Yet it also creates a sense in which non-human entities are active subjects (as in the phrase "letting yourself be danced"), and this moves towards a more nuanced and posthumanist understanding of ecofeminism (e.g.Plumwood 1993; Haraway 1991). In terms of ritual and the performance of human-environment relations, the Women Dancing event is particularly interesting for how it resonates with Schafer's (1991, 97) comments regarding the importance taking "theatre" outside:

We need to breathe clean air again; we need to touch the mysteries of the world in the little places and the great wide places; in sunrises, forests, mountains and caves and if need be snowfields or tropical jungles. For too long the clement temperatures of our theatres have neutralized our thermic sensibilities. Why not a concert under a waterfall or a dramatic presentation in a blizzard? And why should we not feel the rain on our faces when we sing or a distant mountain throw back 
to us the voice we have just sent out to it? Why do we fail to notice the grass at our feet, the darkening of the sky or the sharp green eyes in the night air? [...] These are the miraculous arenas of living drama inviting us to interaction; and the experience is absolutely free

In this context we may understand Women Dancing, and Cae Tan's biodynamic rituals, not only as performances of gratitude but also in terms of facilitating embodied, sensuous experiences of being outdoors. This is a subject to which I return in chapter 5, which explores Emergence's approach, and the role of art, in more detail.

As I set out in the introduction to this chapter, it seems that now more than ever the Anthropocene calls for ways of cultivating different ways of thinking and acting "that differentiate and distribute agency as an essential development in our collective survival on this planet" (Larsen and Johnson 2016, 161). What is perhaps less clear is the role of spirituality in this. Szerszynski argues that not only does the Anthropocene need to be "decolonised", but it needs to be "desecularised", too. He suggests that rather than the Anthropocene representing a loss of sacrality (as is often claimed), perhaps this coming epoch might in fact involve a great acceleration of spirit (Szerszynski 2017, 253-4). Szerszynski takes his cue from the proliferation of concepts such as quantum theory and thermodynamics which are increasingly being invoked with regards to the "interlocking gradients and flows of energy, value, power and entropy" which cross-cross the Earth, and which might yet, he suggests, help us "to grasp the heterogeneous, shifting and contested nature of the Earth's spiritual body, and thereby to start to develop a post-secular analysis of a dynamic planet" (Szerszynski 2017, 255). Spirit, here, is understood to refer to any "embodied or disembodied non-human agency that is experienced, interacted with or is otherwise socially consequential" (ibid.). Szerszynski proposes six possible "Olympian Gods of the Anthropocene" that "clash furiously in the contemporary geophilosophical imaginary". Szersynski suggests that the first "God" through which complex processes of planetary change are being coded is the Anthropos ("the elevated, abstract human as the full, clothed body onto which all flows of matter and value in the new epoch of the Earth are recoded and inscribed. This is the abstract human as despot ..." (ibid. 258)). The second challenger is capital (for some "the real agent behind the transformation of the Earth"). The third high deity is the one which I would like to highlight here in relation to Cae Tan (although there is a fourth, the sun, which I discuss briefly in text box 4.2.2): this is the Earth conceptualised as living being, for example, as Andean deity Pachamama, the "dynamic living system" or "Mother 


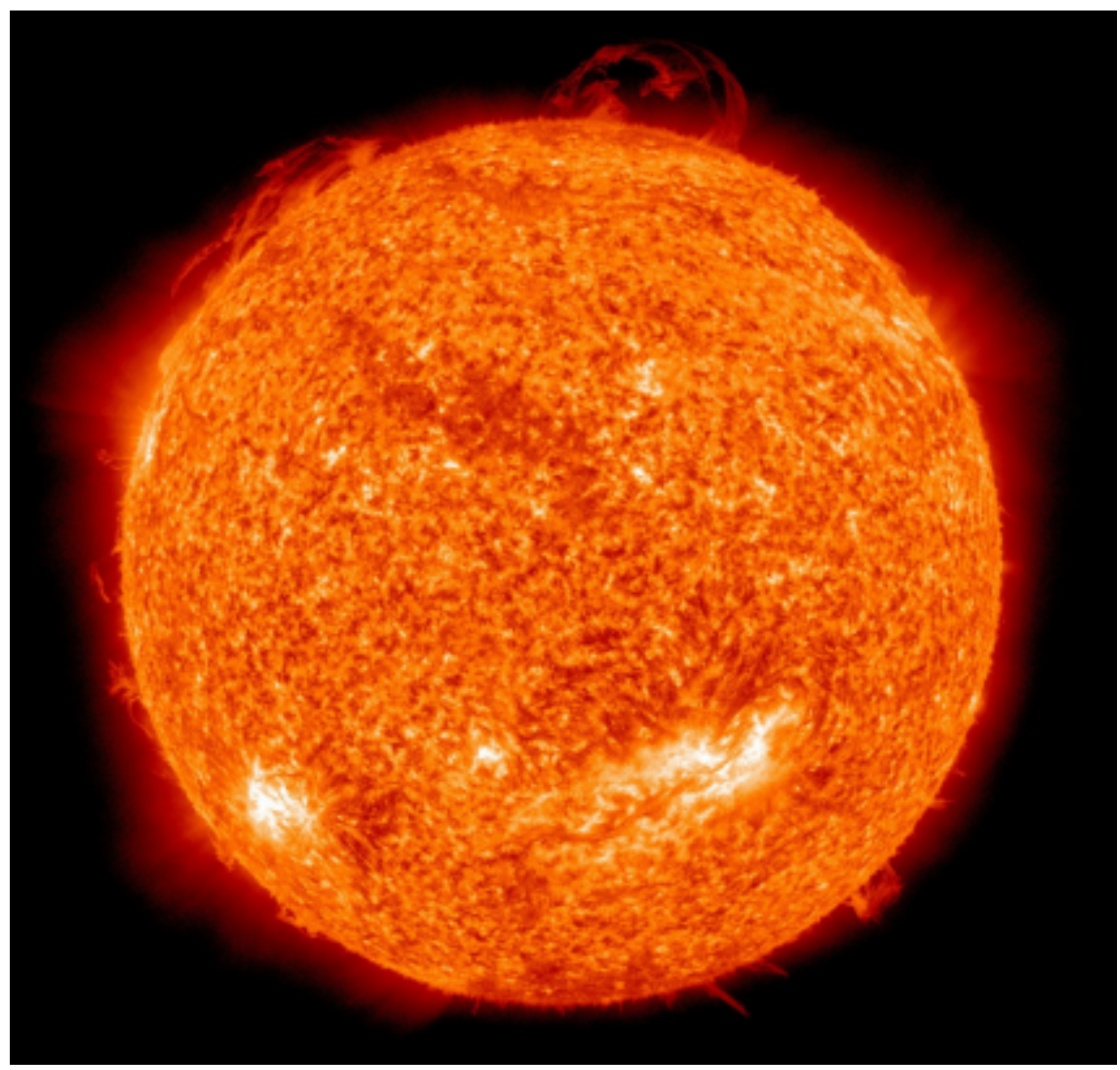

Figure 4.3: The Sun: one of the opening images used in Zero Carbon Britain's presentation to visitors, called The Extraordinary Story of Human Beings and Energy. Source: ZCB

Earth" whose rights are now protected by Bolivian law (De la Cadena 2015). Or it is Gaia, a geophysical Earth whose parts work together as a living organism (Volk 2003).

Szerszynski suggests that to imagine the Earth as such is to imagine some sort of spiritual agency a "planetary spirit" in the making of this new Anthropocene epoch. Biodynamics is a method which conceptualises and performs the Earth as a dynamic living system with multiple more-than-human agencies at work within it. What Szersynski's analysis offers, I think, is a way in which we might understand place-based, improvisatory rituals and ceremonies, such as those associated with Cae Tan, in the wider context of the "grand narratives" and geophilosophies emerging in the wake of the Anthropocene concept. There are some confusing contradictions and tensions 
apparent in this "spiritual turn", for, while the Anthropocene concept asserts humans as a geologic force on Earth, these spiritual practices seemingly attempt a (renewed?) sense of humility and sacredness regarding the more-than-human world. Nonetheless, my analysis of Cae Tan's use of ritual, and of similar approaches by Emergence and Zero Carbon Britain (see text box, below), lends support to Szerszynski's (2017) claim that the coming epoch might, in fact, be one noisy with spirits (earthly and cosmic) as people try to navigate this somewhat confusing terrain.

\section{Text box 4.2.2 Zero Carbon Britain and the Sun God}

And then we discovered coal a massive deposit account containing millions of years of concentrated ancient sunlight. Suddenly humanity was no longer limited to an annual ration of solar energy. (from The extraordinary story of human beings and energy by Paul Allen, ZCB project co-ordinator, in Neal 2015, 20)

Fossil fuels have been essential in that, they've been like the yolk of an egg it's energy from the sun that's been captured and stored that we've used to transform how we live, but we now have to, like the chick, recognise that we've used all we can, it's all gone, we have to switch and burst back out into the sunlight and learn to live off the sunlight. (Allen, speaking at the Small Is festival at the Centre for Alternative Technology in 2015)

Zero Carbon Britain (ZCB) has at its core a conviction that industrialised, resourceintensive societies need to change their relationship with energy (see Allen et al. 2013, 138). To assist in such a change, ZCB endeavours to contextualise current energy use in a longer time scale of human history, and even geological time, to show how what is often thought of as "normal" isn't necessarily so. ZCB tends to frame this as a story, with the sun as its central character. Typically, a presentation to the public starts with an image of the sun (fig.4.3), and a description of the emergence of photosynthesising life forms on Earth, and goes on to tell a potted history of how the sun's energy has powered life, eventually human life, through millennia. The "take home" message of the presentations, as the quotations above suggest, is that, since the industrial revolution, humans have been thriving off a massive "deposit account" of concentrated ancient sunlight, which is now fast running out. ZCB's vision is thus one which imagines a renewables rev- 
olution in terms of a renewal in humanity's relationship with the sun itself, and the "annual budget" of direct sunlight it provides (be that manifested through solar, hydro, wind, or any other renewable energy form), rather than stored deposits of carbon.

In ZCB's account, the sun (re)appears as something of a "high god", to use Szersynski's expression, a gift of energy, the "ultimate capital" (Szerszynski 2017, 259, drawing on Bataille's (1988) theory of "general economy"), until the time that it extinguishes itself (Serres 1982, 169 174). Thus

It is the sun whose lesson of giving without benefit or return was not heeded by industrial society, and it is because of this not-heeding that the buried gift of sunlight thickens the air; that the colossus of the Anthropos has grown into masses, volumes and plates which weigh down on the Earth and alter its systems (Szerszynski 2017, 259)

By evoking a sense of awe and dependence on the sun, $Z C B$ reminds its audiences of the contingency and limits of human agency, because "extinction the prospect of solar death, the heat death of the universe and ultimately the end of space-time itself destroys the 'manifest image' of the human (or even the transhuman) in terms of personhood and intentional agency" (Szerszynski 2017, 261). ZCB therefore makes sense of the Earth's ongoing transformation through the story of the sun, a move which serves to trouble the notion of the Anthropocene as "the age of the humans", and evokes a sense of supernature which was "supposedly banished as a source of agency and meaning by the onward march of modernisation" (Szerszynski 2017, 253).

\subsubsection{Hocus Pocus}

Despite recognition of spirituality as an important aspect of human environment relations, there is often a widespread reluctance to name it as such: even where morethan-human agency and vibrancy is talked about, there is a tendency, particularly in Western discourses, to avoid talk of "superstition, animism, vitalism, anthropomorphism, and other pre-modern attitudes" for fear of "tainting the rationality of secular humans with the stain of pre-modern magic" (Sundberg 2014, 5). I detected a similar reluctance with Cae Tan, too. For all that I have already said about how Cae Tan's biodynamic method performs human environment relations in particular 
ways, it is notable how this seems to be a rather "back-stage" performance (see Goffman 1959) there is a reluctance, or embarrassment, perhaps, to put the biodynamic aspect of what it does in the spotlight.

Cae Tan volunteer, Geoff, with reference to human environment relations more generally, told me that he tends not to go into the "spirituality stuff" when he speaks with large groups of people because it tends to "turn people away", adding that he feels people increasingly need these issues to be presented to them in secular ways. Tom has also mentioned to me that he prefers not to "force" the biodynamic aspect on people it's there if people want to know more, but in terms of publicity he is concerned it might put some people off, or at least make Cae Tan feel less "accessible" to some groups of people than it might otherwise do. This hesitance comes through in Cae Tan's public-facing communications: the biodynamic approach to farming is rarely mentioned, and if it is, it is only by name (for example "We grow and supply fresh, seasonal, biodynamic produce to our members weekly throughout the year"14), without a description of what biodynamic agriculture actually entails, or its philosophical roots. Instead, Cae Tan's "front-stage" performance of human-environment relations revolves far more around the Community Supported Agriculture (CSA) model.

Although CSA and biodynamics are a compatible fit with one another (as Ingram (2007) observes, a CSA arrangement allows a biodynamic grower to contribute to more general spiritual health by growing nutritionally complete food and providing it within the supportive, co-operative framework of a CSA community), the narrative which accompanies CSA carries none of the spiritual "baggage" regarding more-thanhuman agency and sacredness that biodynamics might be seen as having. Nowhere in Cae Tan's regular newsletters, website, or BBC radio Wales interviews (of which there have been a couple) are the words "spiritual" or "sacred" used, despite them appearing in most descriptions of biodynamics online (for example the Biodynamic Association of North America, ${ }^{15}$ the Biodynamic Association of the UK, ${ }^{16}$ and Organic Wales ${ }^{17}$ ). The CSA model features much more prominently in Cae Tan's communications with its members and to the public. In particular, features of CSA such as closer relationships with food, with other members, and between consumer and

\footnotetext{
${ }^{14}$ Available at http://caetancsa.org/en/. Last accessed 16 June 2017.

${ }^{15}$ Available at https://www.biodynamics.com/what-is-biodynamics. Last accessed 17 June 2017.

${ }^{16}$ Available at https://www.biodynamic.org.uk/. Last accessed 16 June 2017.

${ }^{17}$ Available at http: //www .organicwales.com/organicwales/what-is-biodynamics. Accessed 16 June 2017.
} 
grower, are highlighted. As Tom explains in a short publicity film about Cae Tan,

CSA is more than just supplying food to people ... we're creating something bigger, we're reformulating how the local food system works. ${ }^{18}$

That "something bigger" appears to be a sense of belonging and care, as a Cae Tan fundraising campaign in 2015 put it,

Above all else we wanted and still want to create a place where natural resources are cherished and valued, where people feel a sense of belonging and ownership of their agricultural, industrial and natural heritage, and where they feel they are creating an ecologically sensitive future. ${ }^{19}$

Indeed, CSA has been heralded as one of the most likely alternatives to "the entire system of industrial farming" (Guthman 2014, 185), because the CSA grower has a direct relationship with members who pay a regular subscription in return for fresh, locally grown (and usually organic) vegetables. The grower thus sidesteps conventional market structures where they are exposed to risks of fluctuating prices or crop failures (the membership model ensures that the grower has a guaranteed income, while members trust that any "bad" weeks will be balanced by "bumper" weeks at other times of the year). In the CSA model, then, community takes centre stage: as Hayden and Buck $(2012,333)$ point out "member participation is critical in establishing and maintaining a connection to the farm that inspires commitment to a particular CSA." At Cae Tan, members have opportunities to meet one another and visit the farm through open days and BBQs, kids' play days, volunteer days, and the annual Harvest festival and occasional party, as well as through regular newsletters and a facebook page for sharing recipes. An online members survey carried out by Cae Tan in 2015 showed that many people identified feelings of "community" and the relationships between food, the grower, and members, as a valuable feature of the CSA model. It is these community-orientated aspects of CSA which, Hayden and Buck (2012) suggest, present possibilities for more ethical relations with the land, a sentiment which Tom echoes in the Cae Tan video:

If you want a different kind of agriculture that cares for the soil, and cares for what goes into our crops, then we need the community to make that happen

\footnotetext{
${ }^{18}$ Available at http://caetancsa.org/en/. Last accessed 27 June 2017.

${ }^{19}$ Available at https://fundit.buzz/FieldGoodCrowdFarming. Last accessed 27 June 2017.
} 
He goes on to describe why this community participation is important:

If we look after our soils, the soil's going to grow healthy plants and those plants are going to be rich in nutrients, and going to give us the nutrition we need

The interesting thing about this is that it reflects much of the philosophy of biodynamics but gives it a slightly more anthropocentric twist. Notions of care and responsibility are no doubt important (e.g. Lawson 2007), but also perhaps obscure the kinds of more-than-human agency (even the possibility the soil "cares" for us) that biodynamics is more attentive to. Indeed, in Cae Tan's descriptions of the CSA model (above), there is very much a sense of humans "in the driving seat", which is somewhat in contrast with the more co-operative performance of human and morethan-human relations which arises from biodynamics. Bennett $(2007,143)$ recognises a similar tension in her analysis of the Slow Food Movement in America (which shares many similarities with CSA):

The Slow Food programme involves taking the time not only to prepare and savour food, but also to reflect upon the economic, labour, agricultural and transportation events preceding its arrival to the market, consonant with a commodity chain approach that chronicles the 'life-history' of a food product and traces 'the links that connect people and places at different points along the chain.' The strength of this method resides in its ability to give consumers better insight into just what goes into their mouths: not only in terms of ingredients such as pesticides, animal hormones, fats, sugars, vitamins, minerals, etc, but also the exploitation of food workers, and the greed of agribusiness and its agents in Congress. But its weakness may be its anthropocentric allegiances, its tendency [...] to figure food as merely a resource or means.

For me, this gets to the nub of the difference between Cae Tan's "back stage" and "front stage" performances of human/more-than-human relations: the front stage "CSA" one largely figures food as a resource around which a community of people can gather and reorganise local food production, and although there is a strong desire to care for more-than-human others (the soil, for example), there are few indications that these more-than-human others are active participants. As Bennett (2007, 145) observes, "this picture of food [...], as a tool to 'be taken possession of', perpetuates the notion of nonhuman materiality as essentially passive stuff on one side of an 
ontological divide between life and matter." In contrast, the backstage biodynamics rituals seem to embody a more enhanced alertness to food as itself an actant within an agentic assemblage that includes (but is not superseeded by) human metabolism, cognition and moral sensibility (Bennett 2007).

It is not difficult to see why Cae Tan might be reluctant to put its biodynamic approach front and centre stage. Although there are favourable research findings about biodynamically treated soils (e.g. Reganold et al. 1993), these successes are often put down to the more "conventional" proven farming methods which biodynamics incorporates, such as organic agriculture, rather than the "wacky" practices themselves. One soil scientist has described the practice of administering herbal preparations as akin to "driving a tractor naked" in terms of its actual effect on the soil (see Ingram 2007, 307). Members and staff of Cae Tan with whom I have had conversations are not oblivious of such accusations. On one occasion when I was listening to Tom talk about biodynamics, he interrupted himself with a laugh and said "it all sounds like witchcraft!" Similarly, speaking to Ant (who leases the land for Cae Tan) on a separate occasion about biodynamics he told me

Yeah I mean it might be all hocus pocus but it's ah certainly not doing any harm!

This raises an interesting point about the "work" that environmental rituals do. Whether one "believes" that the effect of biodynamics on the fertility of the land comes from a physical, chemical interaction of the herbal preparations, or that the effect comes from people's intentions "imprinting" on the water, is, therefore, not of central importance. Tom happens to believe that the effect comes from both, while other members and volunteers are indifferent they appear not to need proof that the biodynamic preparations directly improve the soil fertility, it is not of primary concern. For example, Ant also told me, referring to the biodynamic method, that

um, it's interesting ... I think, yeah, I think it's kind of good ... I mean even if [people don't] have a sort of appreciation on that kind of spiritual plane ... they always appreciate how sort of intuitive they are about the land and about the relationship between the plants [...] I mean life's too full of ... we're just obsessed by doing rational things, and I find it kind of quite a ... creative ... release. You know I think I like doing things for no rational reasons ...

Another Cae Tan member, Clarity, also told me: 
... I mean, there's so many things in life that you can't understand or try and like ... you know, things that don't make sense, but they don't need to make sense you know? Like, if it works and you see the results of it then, you know OK, I don't understand how a little cow's hoof [...] it's best [not to] try to have to make sense of things, you know? Takes all the magic out of life if you have to always make sense. So um ... I keep my mind wide open! And say 'yeah, bring it on! sounds good to me!'[...] It's definitely not doing any harm!

There are two interesting things to note about this. The first is that, in terms of Cae Tan's "front-stage" performance, there is a reluctance to broadcast much about the spiritual basis of biodynamics, despite this forming a core aspect of its practices. This is perhaps not surprising given that, although census data suggest a high degree of belief in spiritual phenomena of one form or another (Bartolini et al. 2016), there is a parallel public (and, often, academic) discourse about increasing secularisation (e.g. Bruce 2002) which might make people wary of expressing their spiritual beliefs more openly. Second, and perhaps more surprisingly, is that, where Cae Tan members are aware and engaged with the spiritual elements of the biodynamic methods, they themselves do not see a need for "proof" of the efficacy of the method. In one sense this reflects an attitude which is, according to Ingram $(2007,304)$, frequently voiced by members of alternative food networks, that "biologically complex systems are very difficult for conventional science to accurately embrace". Rituals therefore seem to occupy a space in which people are willing to suspend their disbelief, but also in which people can express their dissatisfaction with, as Ant and Clarity say, a purely rational explanation of the world. Sullivan $(2010,126)$ recognises such practices as "counter-powers" to dominant modes of environmental governance which tend to silence more embodied relations and perceptions of more-than-human-others (more on which later). This also resonates with Grimes's (2003) point in the previous section regarding how the beneficial impact that rituals have resides as much in the psychological, bodily, and social (and political) connections they perform and instil as much as in measurable, material outcomes.

The participants in the quotes above seem to engage with the biodynamic rituals as a form of "communication with the world [that] is taken as an end in itself, as the dimension of grace in our lives, rather than as a means of securing our safety and good fortune" (Mathews 2003, 68). Coming back to the theme of sacredness, the quotes also allude to a sense of mysteriousness, and a "presence that presents itself as an absence, a nagging question, a distant calling whose contours remain wholly 
obscure" (Rose 2010, 509). It may not be clear what the ritual does, if anything, but that's part of the point there is a sense in which some things are beyond explaining, and, for Ant, Clarity, and Tom, at least, that's OK. Rituals which perform a sense of the sacred, Rose suggests, offer one way to "listen" to what is otherwise silent and unobservable. Put another way, rituals and performances such as these provide "a language, by means of which unexpressed, and otherwise inexpressible, psychic states can be immediately expressed, $[. .$.$] at the same time making it possible to undergo in$ an ordered and intelligible form a real experience that would otherwise be chaotic and inexpressible" (Lévi-Strauss 1963, 198). However, as Levi-Strauss observes (drawing on his analysis of shamanistic medicine rituals), far from being purely "superstitious", such rituals, by expressing psychic states, can have "real" physiological outcomes, too. Grimes $(2003,44)$ thus concludes that people do not perform rituals merely to exercise limbs, impress people with their skills, or even to demonstrate sacredly held beliefs, but rather, they do it "to discover ways of inhabiting a place. This is the noetic, or the divinatory, function of ritual; ritual helps people figure out, divine, even construct a cosmos." The biodynamic rituals can be thought of in terms of attuning to those more ephemeral or mysterious aspects of "nature", but also to do something in it, live in it, act in it, and transform it a kind of belonging that, Naess (1989) contends, is the starting point for political deliberation. Cae Tan members such as Ant and Clarity are prepared to put their doubts about biodynamics aside, in part, as a political act. As Clarity put it to me,

I feel like ... well ... the world needs hope right now, and the world needs inspiration, and the world needs positive vibes, and it needs to feel like, amongst all of the chaotic stuff that's happening, that there is something good going on. And even if you bumble your way through it or you're not quite sure, the fact that there's still some aspiration um, the fact that you look towards the dreaming instead of ... concentrating on the negative

Here, Clarity is also voicing Grimes's own suggestion that, regardless of one's doubts about "hocus pocus" rituals, "to wait, hoping for certainty [about whether rituals 'work'] before acting, is a greater risk than hoping to learn by acting" (Grimes 2003, 44).

The question of how best to grow food is a central one in the context of socioecological transformation, both in terms of producing enough of it and distributing it equally amongst the world's population, and in terms of meeting these needs without further degradation of soil health and biodiversity. My analysis of Cae Tan's approach 
to agriculture in this section highlights the importance of considering cultural and spiritual dimensions of agriculture, as well as technical practices and scales, when it comes to the potential "sustainability" of particular methods (Peterson 2016). As LeVasseur (2016) points out, agriculture is never simply a technical exercise for the procurement of food. It is also a daily exercise of nurturing and receiving, and can be an embodied and economic expression of people's relations with more-than-human others. CSA and biodynamics represent clusters of ideas which are marginal but highly mobile and seemingly on the increase, what Ingram $(2007,309)$ calls "generative networks" of relations between biological, energetic, spiritual, and human entities which are forming new "nature-cultures" or "imbroglios" (see also Smith 2017). Cae Tan, like many alternative agriculture schemes, espouses a general sense of "going back to" or "reconnecting" with nature, which in some ways, as Ingram notes, can represent a conservative idea of "nature". But it can also, as I have shown, present some more progressive ideas about humans and non-humans as co-participants in agentic assembages, a stance which moves away from the image of materiality as "inert, brute matter" (Bennett 2007, 134) towards a more lively understanding of interdependence. At Cae Tan, these images seem to come in and out of focus depending on which "performance" you choose to participate in (the CSA one, the biodynamic one, or both), or whichever one Cae Tan chooses to present. Consequently, if proponents of alternative agriculture describe themselves or are described as "working with" or "dancing with" nature, for example, it is useful to keep in mind the question "who leads, and when? Who decides the music?" (Ingram 2007, 310).

\subsection{Welsh Government}

Cae Tan is not alone in its desire to "connect things up". The Welsh Government, too, have made interconnectedness a central theme of their policies in relation to sustainability and environment, as evident in a range of policy outputs, from the One Wales: One Planet (2009) title of the Sustainable Development Agenda (giving a sense of unity and wholeness), to the "collaborative", "joined-up", and "integrated" approaches being promoted by the Well-being of Future Generations Act (2015). In fact, Jane Davidson, the former Welsh Assembly Minister for Environment, Sustainability, and Housing, who oversaw the introduction of the One Wales: One Planet Sustainable Development Agenda in 2009, told me in an interview that "I always take a kind of systems analysis of everything I do" and this was one of the reasons she sought to make sustainable development a central organising principle of govern- 
ment. In relation to the Well-being of Future Generations Act and mandatory Future Trends reports, Andrew Charles, the Head of Sustainable Development for the Welsh Government told me that

one of the bigger challenges that we've identified through this process has been [...] ignoring the interconnections between certain issues, whether it's poverty, or health, or climate change, you know, now, whilst we compartmentalise all these policy issues they all, on the ground, out in the street, they all link together they're not sort of divided out, but they've been divided internally. So it's an attempt to bring all that information together, really.

Thus, the Well-being Goals (2015) are designed to cut across various areas of policy which have traditionally been kept apart in silos, and public bodies are expected to work towards all of the goals, not just a select few.

The Welsh Government's approach seems to correspond to a current vogue for systems approaches or so-called "nexus thinking" in policy making. ${ }^{20}$ In some ways this represents, perhaps, a progressive turn to more relational, intersectional thinking which feminist and (more recently) materialist theorising has long advocated. The adoption of nexus approaches by policy has also been viewed somewhat more sceptically (e.g. Allouche et al. 2015). Similarly to the concept of "resilience" (see chapter 3 ), the idea of interconnection and integration is difficult to disagree with, and yet there is little consensus about what it actually means and how it is practised (ibid.). Mansfield and Doyle (2017) note that the concept of the Anthropocene encourages nondualist ideas of humans and nature as always interconnected, but that this in turn unleashes new forms of politics, "particularly regarding efforts to engineer a range of new natures, including bodies, ecosystems, and the earth system writ large" (ibid., 22). When it comes to environmental governance and policy, then, the task is not simply to celebrate that nondualism is being talked about, but to investigate how it works and with what effects, and, with this in mind, I now turn my attention to examine the Welsh Government's rhetoric of interconnectedness and its construction of human environment relations.

\footnotetext{
${ }^{20}$ See, for example, the UN General Assembly thematic debate 'Sustainable Development and Climate Change: Practical Solutions in the Energy- Water Nexus', 16 May 2013
} 


\subsubsection{Environmental limits}

If we trace the origins of the Welsh Government's Sustainability Scheme (and subsequent Well-being of Future Generations Act) back to its origins, we find that, like many environmental policies today, it has a lineage which extends from the publication of the influential report Limits To Growth: A Report for the Club of Rome on the the Predicament of Mankind (Meadows et al. 1972). Limits To Growth proposed that growth was inescapably limited by the physical carrying capacity of the earth, such that voluntary limits should be placed on capitalist growth to avoid dangerously transgressing them (Rickards et al. 2014). In the wake of this proclaimed "apocalyptic horizon" (Dryzek 2013, 37), an era of "green politics" emerged which culminated in the United Nation's decision to establish the Brundtland Commission, whose report, Our Common Future (1987), coined and defined the term "sustainable development". The Brundtland Report was groundbreaking in its focus on the linkages between environment, society and economy, as well as on intergenerational rights, and, following Limits to Growth, it founded its understanding of the unfolding environmental crisis in terms of human "needs" and environmental "limits", and posited sustainable development (as opposed to economic growth) as a way to balance the two. A few years later, in 1992, the UN Conference on Environment and Development produced an action plan known as Agenda 21, and this became the framework through which the idea of sustainable development found its way into the legal and constitutional frameworks of local and national governments. The surge in green politics during the $1970 \mathrm{~s}, 80 \mathrm{~s}$, and $90 \mathrm{~s}$ therefore had at its core the original warnings of Limits to Growth a focus on the finite resources of the planetary system, limitations on the degree to which the planet could absorb humans' pollution and provide for their activities, and the limits to growth and development that these planetary conditions would necessarily entail.

This way of framing the environmental crisis has been a cornerstone of the environmental movement and, indeed, several senior figures in the Welsh Government "cut their teeth", so to speak, during the 1980s and 90s, and have attested to how the Brundtland Report and Agenda 21 influenced their early political careers and their approach to sustainable development in their roles in the Welsh Government. ${ }^{21} \mathrm{~A}$ language of limits features heavily in its environmental policies. One of the overarching principles of the Welsh Government's Sustainable Development Agenda (2009)

\footnotetext{
${ }^{21}$ Comments made by Matthew Quinn, Director of Environment for the Welsh Government, in a public lecture at Swansea University College of Law, 22nd October, 2015.
} 
is stated as "living within environmental limits", and the more recently published Well-being Goals state an intention to be "an innovative, productive, and low carbon society which recognises the limits of the global environment" (Welsh Government $2015,6)$. This appears to track a recent resurgence in the language of limits, led, in particular, by the Stockholm Resilience Centre's work on Planetary Boundaries and its definition of a "safe operating space for humanity" (Rockström et al. 2009) terminology which has also found its way into the Welsh Government's publications.

The proclamation of limits might seem like a common-sense response to environmental problems which appear to be the result of unfettered production and consumption, providing a framework for "sustainable" societal development (e.g. Steffen et al. 2015), and indeed it can be argued that limits provide generative interfaces that are necessary for human innovation and thriving (Kagan 2014). However, Beck (2010) contends that a focus on limits is politically suicidal because it directs people's thinking only to preventing the bad, rather than creating the good. Take, for example, the kinds of verbs usually associated with a language of limits: "stop", "restrict", "reverse", "prevent", "regulate" and "constrain" (Nordhaus and Shellenberger 2007, 7). These words or similar are all to be found within the pages of the Welsh Government's Sustainability Agenda, where there is a strong focus on the need to "stabilise, then reduce" various consumption practices, from carbon to water. Latour (2008), summarising work on post-environmentalism by Nordhaus and Shellenberger (2007), describes how concern for the environment has thus been made into a question of "learning our limits", and is why the ecological crisis has not been met with the same "enthusiasm, energy, optimism, ideals and forward looking democratic spirit as the past tragedies of poverty, tyranny and war" (Latour 2008, 2). It is also ironic that according to Latour just as we are finally bridging associations between humans and non-humans in political and emotional arenas and beginning to realise that the first great narrative of modernisation (separation from "nature") is "complete bunk" (the concept of the Anthropocene signifies this, if nothing else), the environmental movement has come along and put the brakes on, believing that the solution lies in a kind of "re-separation" of culture and nature. In other words, the notion of limits (and a reactionary discourse that usually accompanies it), therefore presupposes some kind of external environment from which humanity has the option of retreating, thus reinforcing a nature culture binary, and a sense in which nature is something separate from, and victimised by, humans. Moore (2015) also suggests that this way of telling the "story" of humanity's relationship with nature (in which humanity has disrupted, defiled and degraded nature), is a particular way of thinking about his- 
tory that privileges what humanity does to nature, and this lends itself readily to catastrophist narratives which are increasingly embraced by scholars, activists, and the public alike.

\subsubsection{Ecosystem services}

Conceptualisations of a problem and efforts to resolve that problem are always closely connected (Moore 2017, 4). Thus, in constructing an imaginary of socioecological relations in terms of what humanity does to nature breaching limits, defiling, and destroying nature the Welsh Government (in common with most mainstream approaches to "Green Thought" (Moore 2015, 29)), creates the conditions for a particular way of framing and responding to these challenges. As McAfee (2016) contends, framing the problem primarily in terms of limits paves the way for "solutions" which are economically orientated, because neoclassical economics is essentially about the administration of goods and resources which are assumed to be in finite supply. Harvey $(1996,131)$ suggests that one of the most striking features of eighteenth century political economy was the way in which capitalism was able to subsume "the cosmic question of the relation to nature into a technical discourse concerning the proper allocation of scarce resources [...] for the benefit of human welfare". In recent decades we have seen this approach compounded by so-called "green neoliberalism", or "neoliberal natures" (e.g. Bakker 2010).

Two clear examples of this kind of economic ideology applied to the more-than-human world although not necessarily explicitly linked to monetary value stand out in the Welsh Government's approach: First, the "ecological footprints" conceptual tool, ${ }^{22}$ which calculates the impact of a person or community on the environment in terms of the amount of land required to sustain their lifestyle, is a headline indicator in the Welsh Government's Sustainable Development Agenda (indeed, its title, One Wales: One Planet, refers to the target of having a footprint that would be globally sustainable, using only one planet's worth of resources). Other publications make reference to the footprint model with phrases such as using "resources efficiently and proportionately" (Welsh Government 2015, 6). The footprint approach to dealing with limits and scarcity draws direct inspiration from the idea that "you cannot manage what you do not measure" (e.g. Suarez 2013), and is, essentially, a form of accounting, conceptualising the environment as a bank of reserves which can be quantified,

\footnotetext{
${ }^{22}$ Available at http://www.footprintnetwork.org/en/index.php/GFN/page/footprint_ basics_overview/. Accessed 24 August 2016.
} 
divvied up, invested in and withdrawn from. This can be thought of as part of wider attempts at bringing "nature" into the economy, alongside other forms of capital, where (the assumption is) it can be subjected to rational management (McAfee 2016, Castree and Henderson 2014). The ecological footprint is an abstraction, a definition of boundaries which allows "nature" to be segregated into typologies, measured (and potentially costed); a process which is deeply authored but comes to stand as authorless and normalised (Robertson 2012). The idea of a "footprint" also raises an interesting contrast with Cae Tan's more lively and engaged imaginary of soil and atmosphere. Moore $(2015,6)$ asks, "is the image of nature as passive mud and dirt a place where one leaves a footprint really the best metaphor to capture the vitality of the web of life?", and in my mind it is also a metaphor which privileges a two-dimensional imaginary (after all, it's the area of the footprint which is deemed to be important), rather than one which encourages a more stratified, depth-aware understanding of lifeworlds (Clark 2017).

The ecological footprint concept is one example of how attempts to "link up" environment, society, and economics might actually result in perpetuating certain dualisms rather than fostering genuine relationality. Another example can be found in the Welsh Government's "Ecosystems Approach", launched in a 2012 Green Paper entitled Sustaining a Living Wales, which set out a new approach to environmental management in Wales. The concept has since been adopted as an overarching framework for the Environment Wales Act (2016), and by the recently formed ${ }^{23}$ Natural Resources Wales (NRW), the government body responsible for environment and natural resources in Wales. The Ecosystem Approach is described as

a strategy for the integrated management of land, water and living resources that promotes conservation and sustainable land use in an equitable way. This approach explicitly makes the link between the services that support life and human well-being and the state of the earth's natural resources (Welsh Government 2012).

The Welsh Government's interest in ecosystems reflects its stated desire to take a more "joined up" approach to environmental matters. Here, there are similarities with Cae Tan in that efforts are being made to understand the interconnections between people and places, and yet the ways in which the Welsh Government presents this idea is markedly different from the ways followed by Cae Tan. For example, the Welsh

\footnotetext{
${ }^{23}$ Natural Resources Wales was formed in 2013, merging three previously existing but separate bodies: the Countryside Council for Wales, Environment Agency Wales, and the Forestry Commission Wales.
} 
Government describes ecosystems in purely "practical" terms, with little indication of the kinds of affects, emotions and energies that might also contribute to such assemblages:

The term ecosystem refers to a community of plants, animals and smaller organisms that live, feed and reproduce in a specific area and the way in which these communities interact with other non-living elements such as the weather, water and soils. Ecosystems are usually defined in terms of their dominant vegetation or environmental features. Describing and defining the natural world in terms of ecosystems allows us to scale the environment to suit our interests (Welsh Government 2012, 1, emphasis added).

One striking thing to note about this language is that it demonstrates a sense of power over rather than power with the more-than-human world; "scale the environment to suit our needs" implies a particular conception of human agency as superior and "in control". In addition, there is no particular recognition that the more-thanhuman world itself possesses any agency, only the acknowledgement that it is alive and humans are connected with it. There is a rather outdated, static conception of an ecosystem which assumes predictability and manageability, rather than a "new ecology" approach that emphasises "disequilibria, instability, and even chaotic fluctuations in bio-physical environments" (Zimmerer 1994, 108). Having said that, in more recent publications there has been a shift in the Welsh Government's language to include a sense of human dependence on more-than-human others. For example, in a 2015 short film about natural resource management in Wales, the narrator describes how

Mountains, forests, fields, rivers, our land and sea provide us with the resources we rely on, to live, work and play. They give us our sense of place, and underline our identity. ${ }^{24}$

This final sentence, "they give us our sense of place, and underline our identity", is one of very few references in any of the Welsh Government's publications to anything more ephemeral about human relationships with the more-than-human world, the "'something there' that cannot be seen" that Rose (2010, 509) evokes. More commonly, the Welsh Government represents its ideas about ecosystems in more tangible,

\footnotetext{
${ }^{24}$ Available at http://gov.wales/topics/environmentcountryside/consmanagement/ natural-resources-management/find-out-more/natural-resources-management-in-walesvideo/?lang=en. Last accessed 22 June 2017.
} 
empirical and scientific terms. As the previous but one extract shows, ecosystems are defined, described, and scaled, conceived of as discreet yet interacting parts ("weather, water and soils") which, often, are represented schematically via a series of connected symbols (fig.4.4). While this might be a well-intentioned attempt at "joined-up" thinking which tries to account for social and environmental complexity, it is worth asking whether simply drawing lines between "objects", or adding words like "interact", actually achieves a more relational account of the world or, in fact, reproduces reductionist and binary thinking. Castree (2003, 280), for example, uses the term "individuation" to refer to "the representational and physical act of separating a specific thing or entity from its supporting context." Putting conceptual boundaries around particular phenomena "involves a discursive and practical 'cut' into the seamless complexity of the world in order to name discrete 'noun-chunks' of reality that are deemed to be socially useful" (ibid.). Similarly, for Alvatar (1993, 185), it is the "splitting of complex ecosystems which simplifies them into legally definable and economically tradeable property rights." Like the concept of the ecological footprint, the Welsh Government's ecosystems approach might also be read in terms of abstraction, in which individual examples are labelled as instantiations of a generic category (e.g. "rivers", "forests") which can be as the Welsh Government says described, defined, and scaled. For Robertson $(2000,473)$, the effect is to sever "the characteristic being measured from the messy uniqueness of the physical site", and can create the impression that things of the same category are exchangeable, tradeable, and replaceable (Robertson's example is the "trade-off" of compensating for the loss of wetlands in some places by their artificial creation in others). What all of these authors seem to be driving at is that "nature", or "an ecosystem", is more than the sum of its parts (Robertson 2012), and that there is something about the materiality of the world that cannot be reduced to mere components. Harvey $(1996,155)$ suggests that there are inherent incompatibilities between the "materialities of nature" and the commodification process, because commodification requires that things are detached from the rest of life. Thus, abstractions (such as footprints and ecosystem components) are necessary in order for them to "bear value in capitalist circulation" (Robertson 2012, 386). With a slightly different take on this tension, Braun (2013, 1) suggests that "the neoliberalisation of nature must be understood, in part, as the strategic containment of the critical energies of new materialist thought" (emphasis added).

To be clear, the Welsh Government does not currently assign monetary value to ecosystems (although Payment for Ecosystems (PES) is something it is currently 


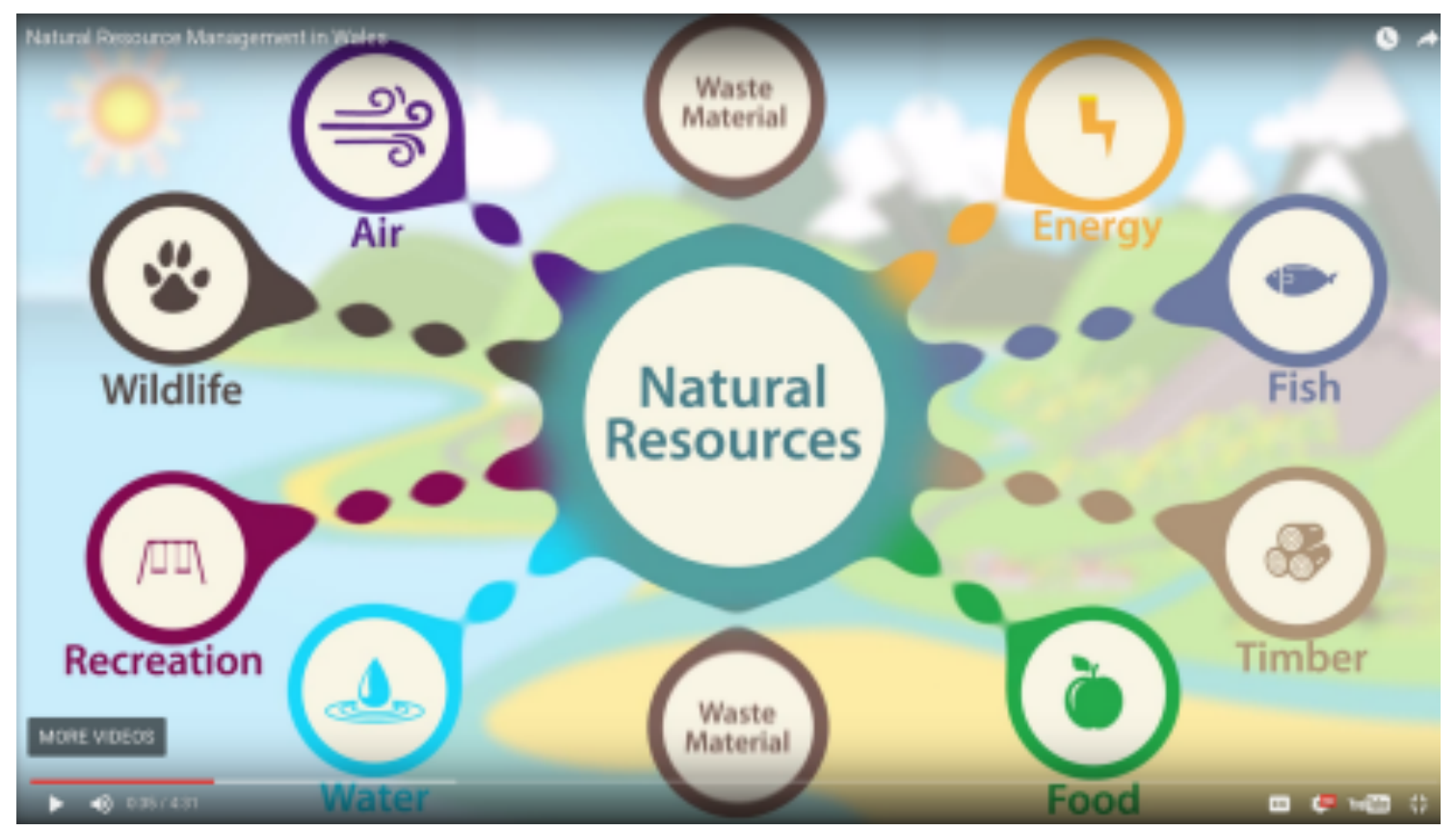

Figure 4.4: A still from the Welsh Government's short film about natural resource management in Wales, depicting a schematic representation of aspects of ecosystems that provide different resources and services.

looking into, ${ }^{25}$ and monetary values are occasionally referred to, as we shall see). But what it does do is use language which constructs a sense in which the relationship between humans and the environment is essentially one of provision and of ownership, supply and demand. The concept of ecosystem services (Costanza et al. 1997) has been much criticised (e.g. Rees 1998, Sullivan 2009, Norgaard 2010), and although the Welsh Government rarely uses the actual phrase "ecosystem services", the "service" idea can be detected not far beneath its rhetoric. For example, one of the opening paragraphs of NRW's statement of its purpose and priorities reads:

Wales's landscape, environment and wildlife are amongst its greatest resource, worth more than $£ 8$ billion to the Welsh economy. But it is even more valuable to us than that. It provides our basic needs, the air we breathe, the water we drink, the food we eat. It gives us energy, prosperity and security; it protects us and makes us healthier and it makes our lives better. (NRW 2013, 4)

In addition, NRW's website explains how

Our air, land, water, wildlife, plants and soil our 'natural resources'

\footnotetext{
${ }^{25}$ Available at http://gov.wales/topics/environmentcountryside/consmanagement/ payments-for-ecosystems-projects/?lang=en. Last accessed 26 August 2016.
} 
provide us with our basic needs, including food, energy, health and enjoyment. $^{26}$

The idea of ecosystem services was initially put forward as an effort to communicate the delusion of economic growth in a way understandable to mainstream economics (e.g. Costanza and Daly 1992, Jansson 1994), by advancing the metaphor of nature as a fixed stock that can sustain a limited flow of ecosystem services, and as a way to build support for conservation (Norgaard 2010). The concept has since risen to become a dominant framework for understanding and assessing ecosystems (e.g. The Millennium Ecosystem Assessment 2005), resulting in what Robertson (2012, 387) observes as a remarkable transformation and normalisation amongst policymakers whereby the "environment [can] be defined as potential commodities in nearly every aspect of its material existence, and at every scale from the atmospheric to the biochemical". Robertson paraphrases the opening line of Capital (Marx 1976): "the ecology of societies in which the capitalist mode of production prevails appears as an immense collection of services. The task is then to discover how such a world comes to be" (Robertson 2012, 387). The Welsh Government's approach has certainly not appeared out of a void. Take, for example, the kind of language used by JeanChristophe Vié, Deputy Head of the Species Programme of the International Union for the Conservation of Nature (IUCN) who, in 2009, stated that "[i]t's time to recognize that nature is the largest company on Earth working for the benefit of 100 percent of humankind and it's doing it for free"; or the Secretary General of both the 1972 UN Conference on the Human Environment in Stockholm and the 1992 Earth Summit in Rio, and the First Executive Director of the UNEP, Maurice Strong's, belief in 1996 that "[i]n addressing the challenge of achieving global sustainability, we must apply the basic principles of business. This means running "Earth Incorporated" with a depreciation, amortization and maintenance account" (both quoted in Sullivan 2010, 116).

When the world is seen to be composed of ecosystem services, Robertson (2012) suggests, the environment achieves a new legibility in the minds of environmental regulators, market designers, development planners, derivatives traders and venture capitalists (as Harvey $(1996,151)$ observes, "to speak in money terms is always to speak in a language which the holders of social power appreciate and understand"). It also creates new social worlds (as Kolinjivadi et al. (2017) contend Payments for Ecosystem Service (PES) serve as a neoliberal performative act). Massey (2013b)

\footnotetext{
${ }^{26}$ Available at https://naturalresources.wales/about-us/what-we-do/how-we-work/howwe-work-natural-resources-management/?lang=en. Last accessed 22 June 2017
} 
argues that, while such "vocabularies of the economy" masquerade as common sense, they are far from being so, and in fact they mould people's identities and relationships in such a way as to give precedence to the very particular economic relationship of buying, selling, and ownership. It also normalises a stance in which the nonhuman world is only nurtured and valued in so far as it benefits humans. It is worth noting here that, although there is one reference to the intrinsic value of life on earth in the Welsh Government's Sustainable Development Agenda (2009, 44), this is presented as a mere add-on to human-centric justifications for action such as "developing the economy", "enjoyment and inspiration", and "benefits to the tourist industry". In reducing the relationship between humans and non-humans primarily to one of service user and provider (as exemplified by the Welsh Government's prolific use of the words "our" and "resources"), it also obscures a more complex, dynamic, and interdependent reality of relations between culture and nature, the human and the non-human, the social and material (Norgaard 2010, Whatmore, 2002). Such an omission creates the impression of one-directional agency, in which humans manage and benefit from "services", rather than a picture of distributed agency that would take into account agency flowing in other directions the influence of ecosystem "disservices" on humans, like crop pests, or disease, for example (Vira, 2015).

\subsubsection{Making nature work}

The portrayal of the more-than-human world in terms of services and benefits leads us to another interesting, and related, effect of the Welsh Government's rhetoric, and this is the implicit emphasis and value placed on the notion of work and productivity. The Welsh Government does not put a price on ecosystem services, but it nonetheless uses a language which puts the environment "to work". For example:

When our environment is working at its best, society as a whole thrives. (Natural Resources Wales, 2017)

Our natural resources are working away, 24-7. They provide us with food, water, and clean air. They break down our waste material. ${ }^{27}$

Wales [aims to have] healthy, functioning ecosystems that are biologically diverse and productive and managed sustainably (One Wales: One Planet

\footnotetext{
${ }^{27}$ Available at http://gov.wales/topics/environmentcountryside/consmanagement/ natural-resources-management/find-out-more/natural-resources-management-in-walesvideo/?lang=en. Last accessed 22 June 2017
} 
Sustainable Development Agenda 2009, 14)

A Resilient Wales: A nation which maintains and enhances a biodiverse natural environment with healthy functioning ecosystems that support social, economic and ecological resilience and the capacity to adapt to change. (WFGA 2015, 6, emphasis added)

This marks, according to Boyd et al. (2001), a transition within capitalist ideology from what was once an extractive relationship with "nature" (the extraction of raw materials), to a move to industrialise bio-geo-chemical processes themselves, and treating them as a form of manufacture. Transforming nature's work/energy into value, Moore suggests, is one of the key ways in which capitalism has survived through projects that compel nature "to work harder and harder for free, or at very low cost" (Moore 2015, 13). So it is that the more-than-human world is reconfigured through the lens of capitalism, a system in which the "capacity to do work' by human and extra-human natures is transformed into value, understood as socially necessary labour time" (Moore 2015, 14). The work/energy might be monetised, or as we see with the Welsh Government's ecosystem approach it might be appropriated via non-economic means, as in the work of a river or a forest. Robertson adds to this picture an understanding of abstractions (like the concept of ecosystem services) as part of a larger process of "rendering surplus value from our bodies and lives" (Robertson 2012, 397), but this is a rhetoric which, even while speaking of valuing nature more, serves to intensify a sense of separation, for it stems from a classical and modern desire for the release of culture from nature (Sullivan 2010). This, too, is reflected in the growing market centred on trading and insuring against environmental risks (e.g. Christophers 2016). One of the darkest sides of this move towards valuation, is that, according to market logic, ecosystem services assume higher market value only when they are in decline, and precisely because they are scarce. ${ }^{28}$

There is a fine line, however, between the kinds of productive "energies" depicted by new materialist thought (for example Bennett's (2009) "vibrant matter") and the "work/energy" being evoked in neoliberal approaches to nature although the former usually is positioned as diametrically opposed to the latter. Indeed, both emphasise an inherent productivity of nature (this, too, is present in Cae Tan's

\footnotetext{
${ }^{28}$ It is interesting to contrast this with Cae Tan's approach in which a fixed price is paid to the grower each week, rather than for the specific quantity or type of vegetables received, and whereby a language of and aspiration for abundance is more commonly associated with its activities than scarcity. For example, its crowd-funder campaign declared that "[Cae Tan] is about a dream that those 15 acres can become an abundant food source". For a "manifesto for abundance", see Collard et al. (2015).
} 
approach). Braun (2013) maintains that there can and must be a crucial ontological difference between the two, a difference which becomes apparent by understanding the neoliberalisation of nature as a response to the critical energies and innovative forces of the more-then-human-world (a response which seeks to capture and contain it), rather than their origin. In one sense it becomes difficult to distinguish between the Welsh Government's and Cae Tan's approaches: the Welsh Government portrays the environment in terms of the services it provides to humans, and Cae Tan, too, is extracting a "service" (food provision) from the land. Both seek to benefit from morethan-human processes (as Braun writes elsewhere, these days "one acts on nature not to change or stop natural processes, but rather to allow natural processes to occur" (Braun 2014, 59)). And yet, for me, there still remains a crucial difference in how the two organisations relate to this productivity which, I think, gets at the distinction Braun identifies. Primarily, this difference is to be found in the contrasting narratives of ownership (Welsh Government) and gratitude (Cae Tan), and in the space allowed (or not) for the "something there" that cannot be seen, a sense of mystery or sacredness which escapes the reduction of an ecosystem into discrete parts, which cannot necessarily be described, measured, scaled, or traded. This is the "creative and sensuous experience of nature" that capitalism renders distant and opaque (Harvey 1996, 126). It is also a matter of how agency is conceptualised, and whether or not anything other than humans are imagined to possess it.

My analysis suggests that the Welsh Government's image of the world is one in which "we see ourselves as utility-maximising and self-interested" and, in addition, which renders "the biophysical world as composed of neatly nested classifications and neatly stacked functions, which takes the appearance of an act of reduction and simplification" (Robertson 2012, 397). This socioecological imaginary, while claiming to be "novel" and "joined-up", reproduces a specifically Western, industrial conception of nature which relies on a deep metaphor of "Earth as machine" (e.g. Gold and Revill 2004), a source of wealth which is constructed of measurable, mappable parts and subject to human domination. ${ }^{29}$ Nowhere is this attitude starker than in NRW's mission statement (2013):

We aim, within a global context, to unlock the potential that lies with Wales's natural resources.

Although there has been a resurgence of more "organic" metaphors about Earth

\footnotetext{
${ }^{29}$ This is not the same, however, as Deleuze and Guattari's (2000) concept of the "machine", which they use as a metaphor for connectedness.
} 
in response to the environmental crisis (Gold and Revill 2004), the Welsh Government's commitment to utilitarian concepts like ecosystem services suggests that the metaphor of the Earth as "a highly efficient and valuable machine" (Daily and Ellison 2002,2 ) remains an influential one. This kind of alienating metaphor (see Merchant 1980) is far more conducive to capitalist modes of consumption and production than conceptions of nature in terms of flows and webs, because capitalist modes of organisation depend on the delineation of objects. Perhaps one of the reasons Cae Tan is able to incorporate emotions such as gratitude and care in relation to what it does is precisely because it organises around an alternative mode of production and consumption the co-operative which does not depend on beliefs about human domination of nature. Cae Tan (along with a growing movement in CSAs) might also be thought about in terms of a response to a sense of loss of contact not only with the natural world, which has arisen from the alienating effects of capitalism, but also as an attempt to reclaim access to land as a means of production (Harvey 1996).

The research presented in this chapter suggests that the Welsh Government is somewhat caught between two rhetorics. On one hand, its Sustainable Development Agenda (2009) and Well-being of Future Generations Act (2015) appear to embrace concepts of interdependency and relationality through an ethics of care and responsibility which extend not only to "distant others" (as implied by the goal of Global Responsibility) but also to future generations. On the other hand, this aspiration falls short of embracing the more-than-human world in a similar way, and instead its imaginaries fall back on deep-seated narratives which reinforce a mechanistic view of the Earth and a dualistic separation between humans and environment. Murdoch $(1997,732)$ pointed out nearly 20 years ago that overcoming dualisms will not simply be a matter of adding terms such as "hybrid" and "cyborg" into our existing modes of thought (or, in the Welsh Government's case, words like "integration" and "collaboration"), "but will require a much more thorough reexamination ... for there is an ever-present danger that the dualisms will prise apart the connections and associations we manage to stitch together". My impression is that the Welsh Government's own imaginaries of socioecological futures are prised apart in exactly this way. Despite its ambitions to integrate environmental, social, economic and cultural realms and, indeed, to make sustainable development the central organising principle of everything that it does, by talking about the more-than-human world almost exclusively in terms of limits, footprints and services, ecological questions still appear as one specific domain of concerns "to be administered" rather than as the core of politics itself. 


\subsection{Concluding thoughts}

Capra $(1996,4)$ contends that many of the world's crises poverty, biodiversity, climate, debt are unified through a "crisis of perception". In this chapter I have taken this insight as my starting point to explore how perceptions of "nature" (and human relationships with it) are constructed and performed through two contrasting examples, Cae Tan and the Welsh Government.

My analysis suggests that Cae Tan embodies some key sensibilities of post-humanism and new materialism, that is, non-duality, relationality, and the perception of humans as embedded within a lifeworld of more-than-human agency. In contrast, although the Welsh Government claims to take a more relational approach, its intentions risk being undermined by some deeply utilitarian abstractions about the environment which lend themselves to neoliberalising attempts to "capture" the productive energy of the more-than-human world, and which shape human identities as "consumers" rather than "participants". This is not to imply a malicious intention by the Welsh Government to disguise its ways of working, but rather to show how relational ideas about "nature" are not per se "good" or "ethical" ones, for they take many forms and compete with (or can be co-opted by) a range of other political agendas. It is also a problem because it suggests that the "relational turn" is being adopted in policy only by name, and not necessarily by content, and therefore these efforts are not sufficient for engendering new kinds of politics a "cosmopolitics", as Stengers (2005) has called it which fully recognise the entangled nature of human and more-thanhuman agencies (see also Latour 1993, Whatmore 2002, Thrift 2008). Cosmopolitics can be described as "a politics of knowledge in which the admission of non-humans into the company of what counts invites new alignments of scientific and political practices and more democratic distributions of expertise" (Whatmore 2005, 93).

Moreover, while the relatively visible, public story that is rehearsed by the Welsh Government falls short of a necessary cosmopolitics, it $i s$ possible to find examples of cosmopolitical stories, narratives, and practices emerging in the "microplaces" (see Cloke and Jones 2001) of life, such as Cae Tan, thus highlighting the potentially rich hybridity of social-natures being forged in and around the places we live, and the importance of looking for them (Cloke and Jones 2001, Jones 2009, Kolinjivadi et al. 2017). However, for both Cae Tan and the Welsh Government, there is also something interesting to be said about their "front-stage" and "back-stage" performances, particularly in the sense that neither are particularly willing to engage publicly with 
notions of spirituality and sacredness, despite in Cae Tan's case this forming a key foundation of its actual practices. In this concluding section I contextualise the findings of this chapter by looking at some of the broader issues at stake in any conversation about human/more-than-human relations, which these case studies have helped to elucidate.

\subsubsection{Vexing vocabulary}

As noted in my introduction to this chapter, language matters because it performs as well as describes (Austin 1975), contributing in no small way to the background "hum" of life (Anderson and Harrison 2010, 7). When Latour asks "why has the question of non-humans failed to enter into politics in any energising way?" (Latour $2008,3)$, the answer is, in part, because just finding the language to articulate entanglements and to get past a nature culture dualism is hard. The theory exists, but notions of human and non-human entanglement and distributed agency are yet to be woven into the "atmospheres" of everyday life. This has become apparent to me nowhere more so than in my own attempts at writing this chapter, throughout which, I note in retrospect, I have relied on vocabulary ("human environment relations", "humans and more-than-humans", "the environment") that, although trying to grasp at a conception of the world beyond a simple nature culture binary, does not go far enough. My own language reproduces what Moore $(2015,13)$ calls "soft dualisms", still "encaged within the prison house of the Cartesian binary" (ibid. 5). This reflects a stubborn tendency to perceive and portray "the" environment as an object. To talk of "humans/society" and "environment", or even "more-than-humans", still risks assuming a fundamental separation between the two (as if they constituted just "two" different categories). When I talk of human environment relations, or interactions, I am still conceptualising two entities that can be separated from each other. I know that they cannot be, and yet my vocabulary continues to reproduce them. The vocabulary of nature "out there", as Moore (2015) points out, is powerfully etched into the socio-cultural DNA of Western societies, and coming up with a new vocabulary and methods is not easy. Throughout this chapter, rather than using a language of humanity's relationship to or with the environment (a conceptual vocabulary which presupposes that these are two separated, but linked, entities), perhaps it would have been more appropriate for me to talk of humanity's relations of nature. I agree with Moore (2015) in his analysis that we need to move beyond the environment as object, to a dialectics of humans in the web of life (see also Harvey 1996), a conception of 
humanity-in-nature rather than humanity and nature. It is easy to recognise the absurdities of the dualisms which structure language, but only to recognise them (and not to try change them) is not enough because this language organises the world in powerful ways ideas are material forces (Moore 2015). Moore's (2015) own suggestion is to use the notion of the oikeios to help show not only that the idea of "humans" and "nature" is obsolete, but that it always has been. He maintains that, rather "than presume humanity's separation, in the recent or distant past, the oikeios presumes that humanity has always been unified with the rest of nature in a flow of flows" (Moore 2015, 12). He goes on to explain that "nature-as-oikeios is [...] not offered as an additional factor, to be placed alongside culture or society or economy. Nature, instead, becomes the matrix within which human activity unfolds, and the field upon which historical agency operates. From such a vantage point, the problems of food, water, oil (and so much more!) become relational problems first, and object problems second" (ibid., 36).

This has interesting implications for conceptualisations of agency, too (something which, in this chapter, I have tended to refer to as either "human" or "more-thanhuman"). In the framework of the oikeios, agency is not a property of nature and/or society, rather, agency is an emergent property of "configurations of human activity with the rest of life. And vice-versa" (Moore 2015, 36). The oikeios was coined by the Greek philosopher-botanist Theophrastus, and is technically an adjective, not a noun, for describing the relationship between a plant species and environment (Hughes 1985). Moore (2015) extends this notion to thinking about the relationship between capitalism and nature, not in terms of how capital and power act upon "nature" (which reproduces a passive notion of nature "over there"), but rather in terms of how capital and power develop through the web of life. Many non-Western and preEnlightenment conceptualisations of the web of life would recognise this approach, which says that the power at stake is not the work that people do on place, but rather the work that place accomplishes through the peoples whose selfhoods are entangled within and constituted through their more-than-human relationships" (Larsen and Johnson 2016, 153, emphasis added). To give but one example, Bawaka Country et al. (2016), referring to Bawaka Yolyu ontology (northern Australia), refer to the notion of co-becoming to describe how all beings, plants, things, affects, and places, are constituted through relationships which are constantly regenerated, "space/place is its doings, its beings, its knowings, its co-becomings" (Bawaka Country et al. 2016, 456). My sense is that, at Cae Tan, the biodynamic rituals are a way of expressing and honouring this co-becoming, even if it is rarely expressed in words (perhaps because 
of a reluctance to "go public" with the spiritual aspects of its practice or, indeed, perhaps because the words to express such co-becoming are few and far between in the English language that Cae Tan predominantly uses to communicate). It could also be, in part, the constraints of language which prevent the Welsh Government from more fully realising its relational aspirations (at least through the public communication which I have analysed here), and instead sees it falling back on existing vocabularies which merely "join things up".

\subsubsection{Thinking differently}

[T] he essence of all our problems is bad thinking, and the only medicine for that is better thinking. (Bateson 1972, viii)

As the Welsh Government case study highlights, portraying "nature" as composed of interacting components can legitimise narrow forms of valuation which are predominantly economic in their focus. Recovering a sense of intrinsic value will require a step away from "conceptions of the status of things" (Naess 1989, 19) towards an understanding of entities or individuals not as bounded but as a point (geometrically defined as having no dimensions) formed by multiple vectors of influences and relations converging at the same junction (Harvey 1996). The crucial and perhaps most difficult part of this is that this has less to do with re-imagining non-humans, and more to do with how we imagine ourselves not as things but as relations. For example, from a deep ecology perspective which tries to get to a place of intrinsic value, "the appropriate frame of discourse ... is not one that is fundamentally to do with the value of the non-human world, but rather one that is fundamentally to do with the nature and possibilities of the self, or, we might say, the question of who we are, can become and should become in the larger scheme of things" (Fox cited in Naess, 1989, 59).

This is not to suggest that the ways in which humans do perceive and value nature are not important to examine, not least, as Robertson (2012) reminds us, because of the current, worrying campaign to redefine the world as an immense collection of service commodities. But it is to acknowledge that the very allocation of value itself relies upon particular anthropocentric mediations and assumptions about the human subject "endowed with consciousness and reflexive as well as practical capacities to become neural mediators of what those values might be" (Harvey 1996, 158). That is, for as long as humans presume to "represent all the values which reside in nature" 
(ibid.), there will always be an idea of human consciousness as somehow emancipated from it (see also Ingold 1986).

Challenging notions of "self" must be central in any effort to de-center the human subject and to move away from ideas of human exceptionalism, domination and control (Anderson 2014b). To imagine myself as a point formed of innumerable intersecting relations with and of other beings and matter not only reconfigures an image of the self-contained "individual", but also expands the notion of "culture" well beyond human knowledge-making and creativity to include all those more-thanhuman forces which participate in these activities, too. Part of this involves, as Plumwood $(2009,127)$ contends, acknowledging that there are "other kinds of minds" (human as well as non-human). But to acknowledge that there are other kinds of minds means, essentially, making one's own mind otherwise, or, put simply, thinking differently (Plumwood 2009).

This task of thinking differently, conversely, may actually, first and foremost, involve not thinking, or rather, accepting and embracing the idea that there are some things in and of the oikeios that cannot be apprehended by humans, let alone valued or appropriated. Concepts like ecosystem services (and the ideologies behind them) seek to make "nature" increasingly intelligible, "a nature that capital can see" (Robertson 2006, 368), and figure the human as a transcendental signifier, operating within what Agamben (2004) calls the "anthropological machine", which always decides in favour of the human and which divides inside from outside: a community of humans on the inside, with non-humans (and some "de-humanized" humans) on the outside, looking in. But Broglio (following Agamben's work on Heidegger, and feminist as well as posthumanist thought), asks whether there could be "an outside beyond the outside that is figured by and for the anthropological machine" (Broglio 2013, 2). It is the entertaining of this possibility, the possibility that "life is a domain which possesses a wealth of openness with which the human world may have nothing to compare" (Heidegger 1995, 255), which presents some possibilities for thinking differently, in as much as it would mean "a hospitality in thought in the zone of non-knowledge or aknowledge. Such hospitality would challenge what constitutes thinking" (Broglio $2013,3)$. There are similarities here with the approaches to temporality and the future discussed in the previous chapter, particularly with regard to how a desire to know, predict, and to make intelligible underpins the Welsh Government's narratives of socioecological transformation. Meanwhile, the more "marginal" projects featured in these chapters indicate some ways in which the unknown might also be allowed to shape our relationships with time and change, and with the more-than-human world. 
Again, perhaps it is not a case of "one or the other", but of making room for both.

Broglio contends that the Academy, with all its cultural apparatus, is foreclosed from non-knowledge and aknowledge. Perhaps one of the reasons I am struggling to find the words to conclude this chapter on more-than-human worlds is because, as Abram $(2014,312)$ suggests, "writing tends to shut out other beings that do not speak in words". Through writing, he intriguingly asserts, we maintain the pretence that humans alone can make sense of what is happening, simultaneously transcribing our "findings" into an almost exclusively human register; "even as we critique it, we reinscribe our aloofness from the animate, expressive earth" (ibid.). This, too, hints at the significance of ritualistic activities as embodiments of particular ways of relating to the more-than-human world that do not necessarily rely on words or writing. Plumwood (2009, 125 126) offers a counterpoint to this, suggesting that writing (literature and poetry, for example) presents some of the best possibilities for helping humans to think differently, for making space in culture for an "animating sensibility and vocabulary", which narrates the oikeios in an active rather than passive voice. Either way, what is clear is that different practices of the self in relation to the "other" are needed, which involve something of a shift away from speculating on existence (as though from afar), to what Sullivan (2010, 126) calls an "embodied consciousness of participating in existence", a way of listening to experiences that express other relationships with more-than-human worlds. To do so can be fraught with potential accusations of romanticism, essentialism, nostalgia or simply cynical dismissal which are difficult to navigate (Sullivan 2010, Plumwood 2009). To my mind it also formulates a question; that is, how to dissolve the dualism which holds nature as external to humanity (a dualism that, as Moore (2015) has pointed out, literally "drips with blood"), while simultaneously accommodating the idea that much of the oikeios will always be external to our thought, and beyond human grasping. In the former proposition externality is something to be banished, in the latter it is to be embraced. This is just one example of the dialectic of thinking through the oikeios which might offer a pathway towards new mental ecologies and ecologies of the imaginary (Guattari [1989] 2014), and towards completely other modes of cognition and experience which can (and do) serve as counter-logics to the logics which separate nature from culture, logics which still loom large in the context of the Anthropocene.

I will try to finish by coming back to where I started: the call to move away from conceptualisations of objects to that of relations has much in common with Massey's (2005) radical re-imagining of space that I outlined in my introduction to this chapter. For example, Naess $(1989,79)$ describes how "a human being is not a thing in an envi- 
ronment, but a juncture in a relational system without determined boundaries in time and space", which resonates with Massey's insistence that space is not "fixed" but made up of a multitude of dynamic relations which are always in process. In addition, Naess's $(1989,59)$ view that "whole and part are internally related" also resonates with Massey's $(1991,24)$ conception of a "global sense of place", whereby places are not "enclosures" with clear insides or outsides. In fact, in his conceptualisation of the oikeios, Moore finds space and nature to mean essentially the same thing: "when geographers say space" he asks, "may we not also say nature? All social relations are spatial relations, relations within the web of life" (Moore 2015, 11). Such conceptualisations of space/nature have implications for ontologies of time and causation, too, for the reimagining of space in terms of complexity and difference, always in process and flux rather than fixed and pre-determined (as discussed in the previous chapter), may give rise to new possibilities and potentialities, and the capacity to imagine that life can be and in many circumstances is otherwise: that there are all kinds of times (and lives) at once. As Massey $(2005,12)$ urges, "for the future to be open, space must be too". However, while I agree broadly with this statement, my analysis in this chapter has highlighted a need to approach claims of "relational" thinking cautiously, and with attention to how they materialise in different circumstances, because in some instances (for example, aspects of Welsh Government's environmental policy), such a rhetoric is complicit with new forms of politics (heralded as "green" or "sustainable") which are poised to be influential in the coming decades, but which mask some fundamentally dualistic and utilitarian assumptions, which merely serve to expand the reach of capitalist/neoliberal atmospheres and ideologies. 


\section{Chapter 5}

\section{The role of art in socioecological transformation}

I decided to stand by the story of art the enchanter of life and alchemist of change. Not an art I was a spectator at or consumed, but an art in which I was a participating collaborator, a story teller and celebrant. An art that inspired the reinvention and reimagining of our world at a time of great uncertainty; an art that could be practised by everyone, inseparable from daily life. An art that had a pivotal role in remodelling society, reinventing its values in a transition to an ecological and less anthropocentric age. (Neal 2015, 4)

$[\mathrm{C}]$ reativity has many different impetuses, paths and visions across the globe. (Eshun and Madge 2016, 779)

\subsection{Introduction}

In the previous chapter I ended with questions of how new ways of thinking and relating to the oikeios might be made possible. As noted, one suggestion is that artistic forms, such as poetry and literature, can be part of such a project by making space in culture for an "animating sensibility and vocabulary" (Plumwood 2009, 125 126). This chapter takes up this topic in more detail, asking how aesthetic forms are being cultivated in response to environmental crises, and what kinds of futures they anticipate and imagine. The research presented here contributes to a growing body 
of work on the role of art and aesthetics in shaping social, economic and political geographies (e.g. Gabrys and Yusoff 2012, Davis and Turpin 2015, Hawkins et al. 2015b, Hawkins 2017), but rather than focussing solely on the effects of particular artworks (e.g. Miles 2010, Hawkins et al. 2015b, Crouch 2015), in this chapter I also ask how each case study reproduces and constructs different ideas about what art "is", and what its role could be, in relation to socioecological crises. I therefore take the case studies as a starting point for critically examining the very notion of "art" who does it, what for, and for whom and I suggest that the form that art takes is just as important as its content when it comes to its transformative potential. The chapter is underpinned by my intention to acknowledge and explore the various forms that "art" and "creativity" take outside of Euro-American/Enlightenment contexts (within which work on art and environment is often situated), and I try to elucidate why such forms might be important for thinking about art's role in socioecological transformation.

\subsubsection{A place for art?}

In Art in the Anthropocene, the editors ask "how can aesthetic practices address the social and political spheres that are being set in stone?" (Davis and Turpin 2015, 3). They contend that the Anthropocene is primarily a sensorial phenomenon, "the experience of living in an increasingly diminished and toxic world" (Davis and Turpin 2015, 1), and that art and aesthetics are central to how humans experience, negotiate and potentially transform these times. Indeed, art has long been theorised in terms of its transformative and revolutionary power. Bloch's (1986) hypothesis was that art reminds the viewer of a latent vision of utopia even in dark times, and many others have afforded a powerful and fundamental role to art in relation to transformation and revolution (e.g. Dewey 1934, Adorno 1997, Marcuse 2007). As Jackson $(2016,10)$ proposes, "creative expression, self-aware concern, and sensual freedom are undoubtedly essential for ethico-political life; there is little, if anything, more important."

In 2005 Bill McKibben famously wrote about the lack of creative response to climate change, asking, "Where are the books? The poems? The plays? The goddamn operas?" Since then, environmentally-themed artwork has proliferated. Prominent examples include Olafur Eliasson's Your waste of time (2013), an installation of pieces of ice from Iceland's largest glacier; Ian Wolter's oil-coated tombstone (2014); 
and Cape Farewell's ongoing project of taking artists to the Arctic in the hope of inspiring them to incorporate messages about climate change into their own art once back home. Research at the intersection of geography and art has also proliferated (see Hawkins 2013 for an overview), involving a host of artists and artworks, including arts science collaborations (Gabrys and Yusoff 2012), land art (Brady 2007), gallery exhibitions (Miles 2010), and participatory art projects (Hawkins et al. 2015b). Art and artists are celebrated for their ability to move the climate change agenda beyond intellectual understanding to emotional engagement (Long Horizons Report 2010), nurture alternative ways of relating to the more-than-human world (Hawkins et al. 2015b), destabilise dominant conceptions of "nature" (Bartram 2005), inspire social change (Wallen 2012), and help people imagine alternative futures (Yusoff and Gabrys 2011).

However, in everyday life and popular culture there is still a tendency to shrug off "art" as a superfluous luxury nice but not essential. Miles (2014) underscores the seeming hypocrisy of his own writing and talking about art in relation to environmental crises: "The script seems to be that, whatever it may mean, the planet will be processed into profit until nothing but dust and ash remain. And I am writing about art." (Miles 2014, 2, emphasis added). I, too, started this research with similar doubts, inclined to wonder whether "making art" amounts to much more than "fiddling while Rome burns", given the magnitude of socioecological crises. Now, however, my views are much more aligned with the position set out by Jackson (2016) (above), that art and creativity are perhaps some of the most important aspects of transformation. In this chapter I hope to show that this change in my thinking has less to do with being convinced of the effects of particular artworks and artists, but rather to do with a more wholesale shift in my ideas about what art is or could be. My thinking in this chapter has been guided by two case studies, Emergence and the Ghost Forest project (an art installation which has been exhibited in London, Copenhagen, and Oxford, and now permanently resides at the National Botanic Garden of Wales in Carmarthenshire). Both projects make claims about the importance of art in relation to socioecological transformation, but in very different ways. This difference might be summed up, I suggest, by contrasting the ideas of "art" and "artfulness" the former being something associated with categories like "artwork" and "artist", while the later is far less delineated or restricted to specific people or sites, and more applicable to everyday life and social activities.

The research that has informed this chapter is based on mixed methods: extensive participant observation with Emergence, in-depth interviews with key people asso- 
ciated with both the Emergence projects and the Ghost Forest, and observations of, and "vox-pop" interviews with, Ghost Forest visitors. This has been supported by document analysis of Emergence and Ghost Forest material online and in print (including visitor comments left in visitor books at previous Ghost Forest exhibits in London, Copenhagen, and Oxford). Although my conversations with viewers and participants have undoubedly shaped my understanding (particularly in the case of the Ghost Forest where I was able to carry out vox-pops), my intention in this chapter is not so much to try to convey what effect the art had on other people (this would be a very psychologically-complex task, involving longitudinal surveys of people's attitudes and behaviors over time, as well as trying to account for the influence of other factors), but is rather to provide a more autobiographical account of the case studies' different approaches to art and socioecological transformation, and my own experiences with them. In doing so, I bring together literature on pluriversal creativities (e.g. Eshun and Madge 2016) and geography, art, and environment, to show how the "work" that art does in the context of socioecological transformation might be reconceptualised. The chapter begins with a discussion of how the Ghost Forest and Emergence approach environmental issues through different perspectives on life and death, and then develops these themes to explore the kinds of "topologies" of the environment each one creates. The second half of the chapter examines some implications for re-imagining agency and subjectivity, and and how this might translate into alternative ways of conceptualising what "environmental art" is, and who does it. Finally, the chapter situates this reconceptualisation by exploring some relations (and tensions) with geopoetics and indigenous ecopoetics.

\subsection{The art of life (and death)}

\subsubsection{A ghostly presence}

The depiction of environmental destruction by Angela Palmer's ${ }^{1}$ Ghost Forest installation is stark: ten huge primary rainforest tree stumps transported from Ghana, their roots intact but whose missing trunks are intended as a "metaphor for the removal

\footnotetext{
${ }^{1}$ Palmer is an artist and former journalist based in the UK. Her artwork features diverse topics, often using MRI and CT scans to to create 3D images. The Ghost Forest is one of several works with an environmental theme, although, as she told me in an interview, she does not consider herself to be an environmental artist.
} 


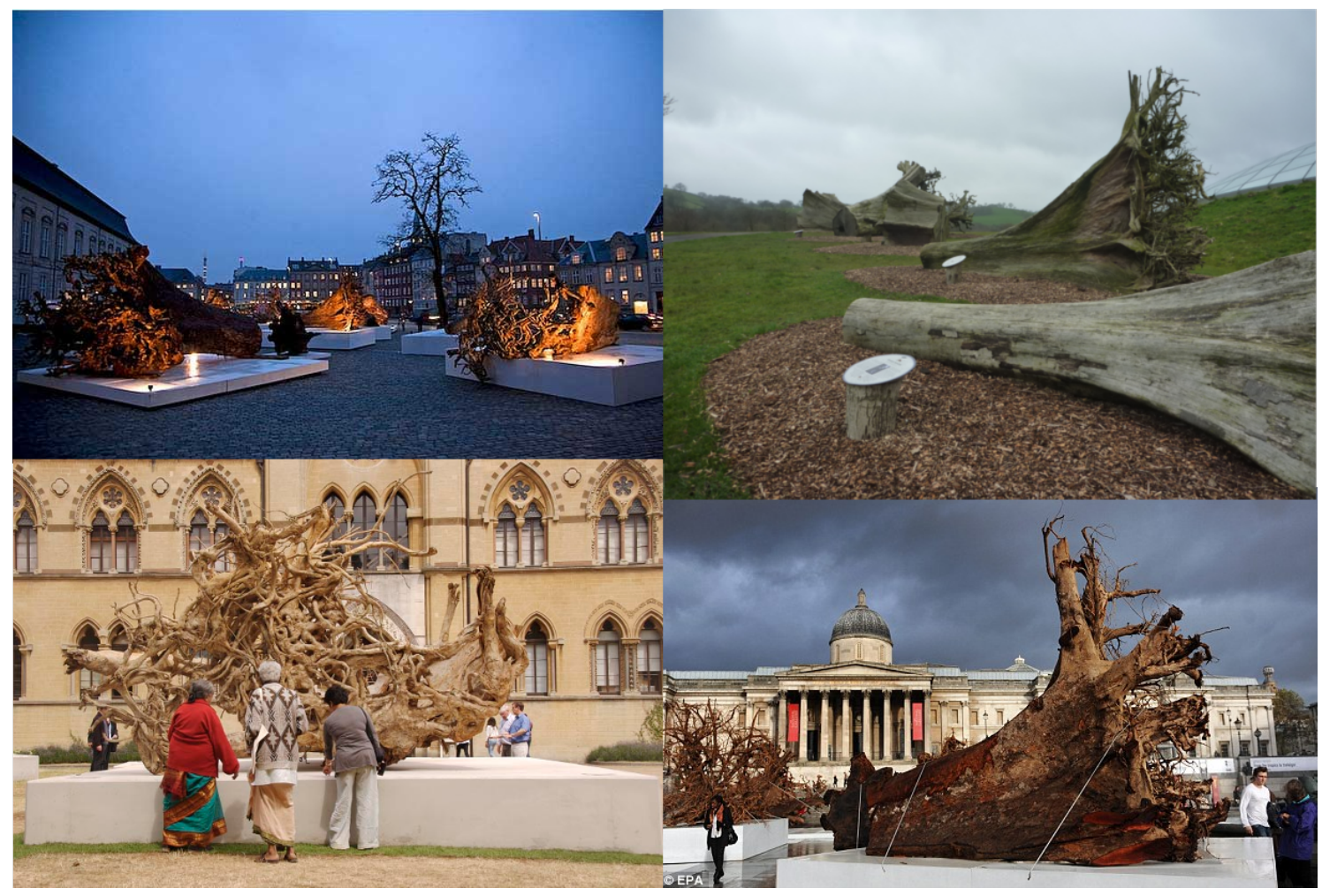

Figure 5.1: Clockwise from top left: The Ghost Forest in Copenhagen, the National Botanic Garden of Wales, Trafalgar Sq in London, Oxford.

of the world's lungs". ${ }^{2}$ The trees have been exhibited around Europe since 2009 (first London, then Copenhagen, then Oxford, and finally to the National Botanic Garden of Wales)(fig.5.1). The work, as the Ghost Forest website explains, is intended to highlight the depletion of the world's forests, and the continued rate of deforestation. ${ }^{3}$ Palmer told me that she was inspired to embark on the project because

I was simply aghast when Andrew Mitchell, a scientist friend, told me a rainforest the size of a football pitch is being destroyed every four seconds. I felt as an artist I should try to create a means of provoking thought about this.

The effect of displaying ten enormous, dead, tree stumps displayed horizontally on white plinths in public places, together with interpretation explaining their origin and message, is as one might expect; as Palmer told me, generally they provoked an acute sense of loss or impending loss in those who encountered them:

\footnotetext{
${ }^{2}$ Available at https://www.angelaspalmer.com/the-ghost-forest. Last accessed 23 October 2017.

${ }^{3}$ Available at https://www.angelaspalmer.com/the-ghost-forest. Last accessed 23 October 2017.
} 
I noticed a lot of people spontaneously hugged their children or their partners this pattern emerged at all locations.

Palmer stresses that she doesn't want to be prescriptive about what people take away from the installation, telling me that,

I don't want to make people feel guilty as I think that's a very negative approach.

She wants people to see optimism in the message that Ghana is now operating much more sustainable logging practices. Nonetheless, visitor reactions imply that loss and sadness, combined with an appreciation for the beauty of the trees, is what defines most people's experiences of the Ghost Forest. The visitor books kept at the exhibition sites in Copenhagen, Oxford and London record comments such as:

I counted four seconds, realised what it meant to have a football pitchsized bit of forest disappear, and nearly cried.

Fantastic display, beautiful trees, makes me say wow, makes me wonder how, makes me pleased we love trees, but makes me cry. Is it too late?

Sad \& beautiful we should all meet these trees who provide us with their natural material...leave them alone!

A travelling arboreal graveyard with fresh remains of the corpses. Shame.

How eerie and beautiful these tree stumps are.

The Ghost Forest is an attempt to represent the issue of deforestation, to bring its apocalyptic spectre to people's attention. Yet, as Grosz (2008b, 75) says, no art is representational, "they assemble, they make, they do, they produce". Marcuse (2007) insists that art and culture shape forces of domination as well as liberation, and Miles $(2016,2)$ adds that art and culture are factors in the "evolution of people's attitudes to each other and to the planet's ecosystems". If this is the case, what kinds of ideas, atmospheres, and affects does the Ghost Forest assemble in relation to socioecological transformation?

I suggest that Palmer's efforts to highlight the devastation of deforestation is indicative of an emerging "sublime of environmental destruction" (Miles 2016, 15), a means of combining beauty and ugliness (Peeples 2011) to stimulate a sense of awe 
and terror at the destructive capacities of humans. This can be contrasted to a traditional notion of the "sublime", in which it is the beauty and power of dramatic landscapes that provokes a sense of awe and terror. As de Botton (2011) points out, the very idea of planetary abuse and environmental crises has fundamentally altered how many humans relate to the more-than-human world. Whereas once humans might have feared "nature's" mystery and power, now we are told that humans are in fact the ones with all the power imposing an influence on everything from the early blooming of daffodils to the mass extinction of species (as the term Anthropocene implies, humans are now considered a geological force). A crucial aspect of this mood is that, implicitly, people are asked to re-conceive of themselves as "unthinking killers", and this shift has been accompanied by a general "hysterical sentimentality about nature" (de Botton 2011, unpaginated). The Ghost Forest, as a project that required significant effort, cost, and logistics to transport ten tree stumps half way across the world, might be seen as evidence of just such sentimentality and nostalgia. The very name "Ghost Forest" carries with it overtones of death, loss, and haunting, with the implicit assumption that it is "we" (a generalised humanity) who have done the killing. Levene (2010) relates a contemporary fascination with death and violence with Freud's notion of the death instinct, or Thanatos; indeed, the idea that humanity is somehow compelled towards death and destruction is reflected in some of the attitudes and comments of the Ghost Forest artist and its audiences. For example, Palmer, reflecting on the prospects of halting deforestation, told me that

Altruism simply isn't in our DNA; self interest and greed comes in spades.

This is a message that is not lost on viewers indeed, it is a view that, in my experience, people were quite likely to express. For example, when I talked to visitors to the Ghost Forest at the NBGW about the issue of deforestation, many responded with themes of (apparently inevitable) human destruction and greed:

I'd like I'd hope but being realistic, no I don't think there will be [improvement], because man is too greedy.

People are too greedy...there's not enough people to get them to stop.

Man has got greedy, and I think, you know, if we can have it, and there is a price, we'll pay for it.

But I just think as long as man is greedy to have everything then, it'll cost the bigger picture... 
I mean...deforestation is a symptom of what we are doing...it's not just the forests, is it, that we are wrecking...all sorts of things.

The Ghost Forest, through evoking a "sublime" of environmental destruction, is an artwork that seems to have been designed, at least in part, as a means of chastisement. This is symptomatic of a situation in which Latour (2008, 9) says "the unwanted consequences [of modernity] are suddenly considered as such a monstrosity that the only logical step appears to be to abstain and to repent: 'we should not have committed so many crimes, now we should be good and limit ourselves"'. Correspondingly, it also hints at an underlying assumption that social change can be brought about through raising awareness about the horror of environmental crises. Such an approach resonates with the "deficit model" of human behaviour, which relates a lack of action to a lack of adequate information, but which has been largely dismissed as insufficient for explaining how and why social transformation occurs (e.g. Moser 2006). I highlight this not so much in order to question what kinds of effects the Ghost Forest produces in its audiences, but rather to point out that this way of constructing the "work" that art does that is, as a means of representing and conveying a message about environmental issues to an audience is but one way of thinking about the role of art in socioecological transformation. In the following section, I contrast this approach with Emergence's, a group whose activities are not underpinned by a desire to convey an overt message about aspects of environmental destruction, but which nonetheless seeks forms of socioecological transformation.

\subsubsection{Eros and art}

In contrast to the Ghost Forest's name, which evokes a sense of death and loss, the name "Emergence" alludes to a sense of life, of continuation and evolution of the new. Indeed, Emergence's website states an aspiration to find ways to "re-enchant ourselves with each other, with art, and with life", ${ }^{4}$ and it is explicit about art being a service towards this end, as its website also states:

Emergence consciously seeks to embody the values we treasure in a hope that a more creative, caring and compassionate planet might be our next evolution, revolution, or re-evolution. Emergence is a collaborative project that advocates creative practice for a sustainable future through

\footnotetext{
${ }^{4}$ Available at http://www.emergence-uk.org/\#the-emergence-vision. Last accessed 31st May 2017.
} 
hosting artful events and gatherings.

Here, Emergence's ethos might be compared with notions of Eros, a force that tends towards a will to live and liberation, and an antagonistic force to Thanatos. In Eros and Civilization, Marcuse (1966) develops a notion of art as something which rejects oppression and alienation, and these ideas, which relate art with something akin to a "life force", resonate with Emergence's approach.

As noted in previous chapters, Emergence's activities centre on group conversations and walks. An example of how Eros features in its approach is in the use of circular and spiral forms (fig.5.2) in many of its activities, shapes that are associated with a "Dionysian" celebration and affirmation of life's rhythms through artistic expression (Leuthold 1998). For example, group conversations are always facilitated in a circular form (as a uniting space in which there is no expressed hierarchy), and during Emergence's Walk That Reconnects (which I joined in 2014), the form of the labyrinth featured prominently: participants arrived, after three days of walking, at Rhossili beach on Gower to find a huge labyrinth drawn (by prior arrangement by Emergence) into the flat sands of the beach at low tide, which the participants were invited to walk (fig.5.2). In addition to the labyrinth, the conceptual framework for the walk (and indeed much of Emergence's other work) followed a spiral form, after a framework developed by Joanna Macy called the Work That Reconnects. This is described as a framework which "repeats itself, as the spiral circles round, but ever in new ways. The spiral is fractal in nature: it can characterise a lifetime or a project, and it can also happen in a day or several times a day." 6 At another event, walkers walked around the Maen Ceti standing stones on Cefn Bryn, Gower, performing an ancient Gower tradition of circling the stones.

Here, there are some similarities with Cae Tan's biodynamic rituals described in the previous chapter. The use of spiral and circular forms has a ritualistic quality. Dewey observes that ritualistic practices, despite being widely denigrated as irrational and superstitious, endure precisely because they are "immediate enhancements of the experience of living" (Dewey 1934, 30), and he goes on to say that "works of art are the most intimate and energetic means of aiding individuals to share in the arts of

\footnotetext{
${ }^{5}$ Dionysian refers to a philosophical/literary concept based on Greek mythology, and is contrasted with the Apollonian. While the Apollonian refers to rational thinking and order, the Dionysian appeals to irrationality, chaos, emotions and instincts. The Dionysian is also often contrasted with Kant's idea of the sublime because, whereas the sublime requires critical distance of the viewer, the Dionysian requires a closeness of experience (see Nietzsche (2000) for an account of the Dionysian and Apollonian in art).

${ }^{6}$ Available at https://workthatreconnects.org/spiral/. Last accessed 8 June 2018.
} 

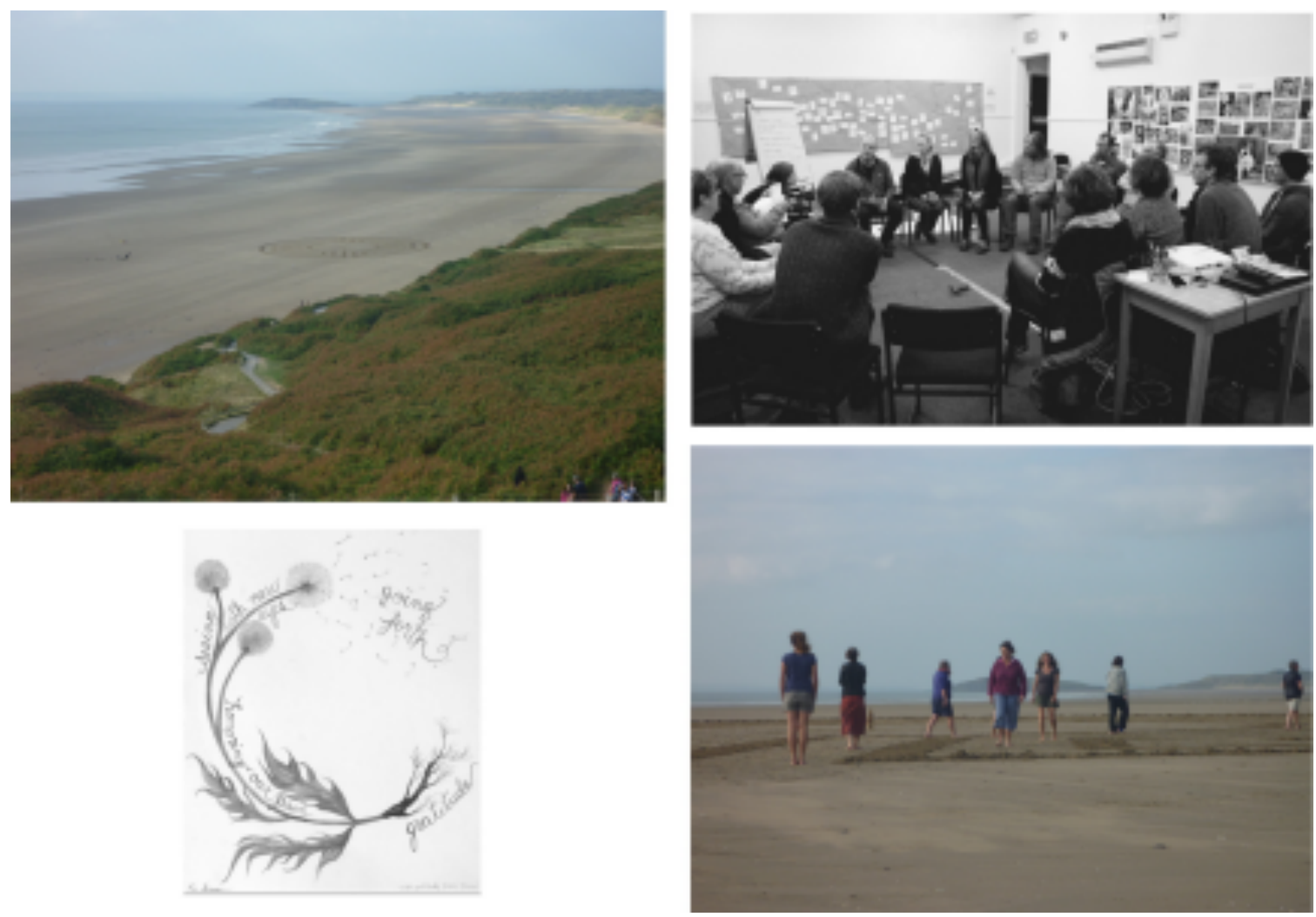

Figure 5.2: Clockwise from top left: the Walk That Reconnects labyrinth on Rhossili beach (source: author's own); The Council method being used during COP123 to facilitate dialogue (source: Eleanor Flaherty); walkers in the labyrinth (source: author's own); an illustration of the Work That Reconnects conceptual spiral, including the stages "Gratitude", "Knowing our pain", "Seeing with new eyes", and "Going forth" (source: Dori Midnight).

living" (ibid. 336), and that these experiences are a "manifestation, a record and celebration of the life of a civilization, a means for promoting its development" (ibid, 326). Similarly, in Alford's commentary on Marcuse's essay Ecology and the Critique of Modern Society, he suggests that Eros needs to be valued not only as a utopian impulse, but also in the here-and-now, "as a reminder of the fundamental reality of the human desire for peace, joy, and happiness. Nothing is more important and valuable than this, which does not mean that these things can only be valued in an all or nothing fashion" (in Marcuse 1992, 48). In this, it is possible to draw out a perspective on the transformative potential of art which does not depend on a sudden or novel "rupture" which might provide new ways of seeing the world, but rather is continuous with life, perhaps arising out of rituals and traditions which are familiar, rather than shocking.

Emergence's responses to socioecological crises group walks, ritualistic ceremonies, conversation may seem odd: they do not seek (explicitly) global goals such as stem- 
ming deforestation or halting species extinction, indeed it doesn't even appear to actively engage with the forms of political and institutional power which perpetuate environmental destruction. Instead, Emergence aims to nurture and honour life at the smaller scales of everyday being. Here it is worth quoting Marcuse at length, because he gets at what it is that might, conversely, be quite radically transformative about Emergence's seemingly inconsequential activities:

What the force of Eros is powerful enough to do is the following. It serves to move a non-conformist group, together with other groups of non-silent citizens, to a protest very different from traditional forms of radical protest. The appearance in this protest of new language, new behavior, new goals, testifies to the psychosomatic roots thereof. What we have is a politicization of erotic energy. This, I suggest, is the distinguishing mark of most radical movements today. These movements do not represent class struggle in the traditional sense. They do not constitute a struggle to replace one power structure with another. Rather, these radical movements are existential revolts against an obsolete reality principle. They are a revolt carried by the mind and body of individuals themselves. A result which is intellectual as well as instinctual. A revolt in which the whole organism, the very soul of the human being, becomes political. A revolt of the life instincts against organized and socialized destruction. (Marcuse 1992, 36 7)

A focus on life instincts might well sound like a romantic, utopian ideal, but Woodyer and Geoghegan (2013), following Bennett (2001), urge that (re)enchantment is perhaps one of the most important ethical-political projects there is, and is by no means a redundant concept in an era where dystopia often seems to rule. The experience of wonder is, they argue, crucial to motivating ethical behavior, and can have a transformative effect on one's perception of, and engagement with, the world (see also Thompson 2009, 136 159). Marcuse identifies art as a vehicle for a revolution in consciousness because "[...] the struggle for an expansion of the world of beauty, nonviolence and serenity is a political struggle. The emphasis on these values [...] is not just a romantic, aesthetic, poetic idea which is a matter of concern only to the privileged; today, it is a question of survival" (Marcuse 1972, 12; see also Gablik 1991).

There is, however, a counter-argument to such an "affirmative" stance, one that questions ontologies of becoming that uncritically propagate an ethos of joy and vitalism. 
Such arguments are concerned with the socio-economic drivers and implications of intensifying expectations for creativity, connectivity, and "compulsory happiness" (e.g. Ahmed 2010, Culp 2016, Osborne 2003). Nonetheless, "enchantment" (if this is a word we can usefully employ to describe Emergence's approach) entails, according to Bennett (2001), an attentiveness to what is disturbing as well as wondrous and affirmative. Emergence's approach to art is one which seeks to honour and celebrate life, but it does so in a way which allows space for vulnerability and disturbance, too. In this respect, neither the Ghost Forest nor Emergence shy away from themes of death and destruction, but the ways in which they deal with them vary. Emergence often invites participants to acknowledge their own vulnerability amidst environmental death and destruction. This is particularly evident in its use of the Joanna Macy Work That Reconnects framework as a structure for walks and for shorter, conversational events. The framework includes a stage for "honouring our pain for the world", ${ }^{7}$ which is designed to allow space for people to attune "to the world in all its particularity, strangeness, enchantment and horror" (Anderson et al. 2012, 213) (for example, by allowing six hours for discussion after screenings of climate change films during the COP123 events in 2015). While the Ghost Forest is also designed to prompt feelings of loss and sadness, my sense is that it is intended to provoke action, more than acceptance. ${ }^{8}$ Emergence, in contrast, starts from the assumption that the most transformative thing to do would be to "stay with the trouble" (Haraway 2016), rather than seek to immediately jump to solutions, for it believes that this can facilitate a deeper engagement with socioecological crises and possibilities for change. Similarly, Smith $(2011,74)$ suggests that the "problem-solving approach denies the ways in which such actions often turn away from the inescapability of pronounced biological, ecological, and geological change". In essence, while the Ghost Forest seems to perpetuate a sense of loss and chastisement, Emergence, following the Joanna Macy philosophy, reframes feelings of sadness and loss for the world not as evidence of human guilt, but as evidence of mutual belonging in a relational universe, and hence possessing the willingness and ability to take action on its behalf. This sentiment is echoed in various guises, for example, Latour's conviction that to construe human actions as "monstrosities" is to prevent the necessary psycho-social conditions from arising. Instead, he suggests that humans ought to "stop flagellating ourselves and take up explicitly and seriously what we have been doing all along at an ever increasing scale, namely, intervening, acting, wanting, caring" (Latour 2008,

\footnotetext{
${ }^{7}$ Available at https://workthatreconnects.org/spiral/. Last accessed 8 June 2018.

${ }^{8} \mathrm{I}$ do not mean acceptance here as in merely "accepting" the situation without question, but rather as a form of deeper "acceptance" of what is happening, and acknowledging our own emotional responses to this.
} 
$9)$.

These different approaches to the "work" of art can be viewed in a more directly material sense, too. Whereas the Ghost Forest intends to "save life" through an abstracted chain of awareness-raising and conservation partnerships, its material existence is arguably much less conducive to life: several of the trees had to be felled (others had fallen in storms), and the logistics of getting to, removing, and transporting ten primary rainforest tree stumps from Ghana to Europe entailed quite some $\mathrm{CO}_{2}$ emissions, for a start. Highlighting this is perhaps a little unfair, especially given the Ghost Forest project's full ownership of these environmental impacts (and efforts to ensure that the trees were sustainably sourced and the carbon emissions offset), but it does get at a crucial difference between whether "environmental art" is deemed to be "environmental" only in its content, or more fundamentally, in its form (Morton 2007). As Steiner has written, we must "detach the values of self-expressivity, excitement, and ecstasy from waste, and attach them instead to sustain-ability [sic]" (quoted in Phillips 2015, 62), and this seems relevant to many environmental artworks, like the Ghost Forest, which are resource-intensive (see Bannon 2011). An alternative approach to art is one which, as already noted, actively supports and maintains life rather than depletes it even if those actions appear mundane and inconsequential in the face of enormous environmental problems. In the next section I elaborate on the different kinds of "work" the Ghost Forest and Emergence do with regards to the relations they construct between humans and non-humans, and how this, in turn, impacts on their constructions of place.

\subsection{A sense of place}

Coming up with a new worldview means dealing with how humans experience their place in the world. Aesthetics thus performs a crucial role, establishing ways of feeling and perceiving this place. (Morton 2007, 2)

Both the Ghost Forest and Emergence are broadly concerned with how humans relate with the more-than-human world, and express a desire to contribute to transforming these relations. In their different approaches to socioecological transformation, it is interesting to see how each constructs contrasting imaginaries of scale and, correspondingly, how this links to different perspectives on dwelling and relating with the more-than-human world. 
The Ghost Forest is underpinned by a certain "globalness": as noted, the project involved the removal of ten tree stumps from Ghana, transported around the world to various sites in Europe. It is intended to bring the rainforest to audiences' doorsteps, and indeed this is a key aspect of the Ghost Forest's appeal. An education programme associated with the Ghost Forest while it was in Oxford was called "I touched the Rainforest" (every school child in Oxfordshire was invited to come and touch the Ghost Forest trees) and now, at the NBGW, part of the interpretation states that the Ghost Forest "has touched people around the world and now you! [sic]". Often it was the sheer distance the trees had travelled, and the connection they enabled with otherwise "exotic" places, that excited or moved people. I overheard visitors at the NBGW exclaim things like "all the way from Ghana!" and "Even to get it on a ship!", and the visitor books at previous exhibition sites recorded comments such as

What a wonderful opportunity to see some exotic wood in its natural form a long way from home.

Thank you so much for bringing these great and beautiful beasts for us all to contemplate.

It's an important message to be taken round the world. A magnificent touch-stone for everyone to experience!

At the NBGW, the Ghost Forest is supported by the Welsh charity, Size of Wales, which coordinates a scheme to protect tropical rainforest in Africa and South America that amounts to an area of 4 million hectares (twice the size of Wales). This further ties the Ghost Forest into a global imaginary, as well as a global network of people and organisations from the project's main sponsor, Deutsche Bank, to John Bitar and Co, one of the largest timber producers in Ghana, which helped Palmer to realise the project. The Ghost Forest's global outlook is perhaps not surprising given that it has become customary to think of environmental crises in global terms; indeed, much of the environmental movement in the European/American context has committed itself to trying to highlight the plight of distant places and others melting ice caps, starving polar bears, and homeless orangutans, for example. Palmer's Ghost Forest like Olafur Eliasson's iceberg installation mentioned in the introduction can be understood as extensions of a mentality that impresses the importance of thinking about our global impact by literally bringing those other places to us, so that we can no longer "pretend they don't exist". On the Ghost Forest website, the (then) Director of the Size of Wales charity highlights the significance of "thinking global", 
saying

Wales is stepping up to the challenge of stopping tropical forest destruction through Size of Wales and having Ghost Forest here in Wales to remind us will really strengthen our resolve and will help us all to get closer to the issue of tropical deforestation.

In some ways this global imaginary seems to heed calls for what Massey (1994) calls a "global sense of place" that is, an ability to imagine the world as a complex network of relations which link us in our kitchen (say), to distant places and others. To do so is to be able to understand how our actions reverberate around the world, and thus to instil an ethic of care and responsibility towards those relations. Beck has articulated something similar in his Cosmopolitan Vision, in which he hopes people will grasp the "social and political realities in which we live and act" (Beck 2006, 2). In this respect, the Ghost Forest is characteristic of a movement in environmental art to provide experiences which disrupt our routines and ordinary worldviews (Miles 2016), whose "otherness" is precisely part of its appeal in that it might expose audiences to new perspectives on the world. It also aligns with more fluid ideas about art and place that artworks can move about without loosing their ability to resonate with or contribute to the changeable nature of place, while also encompassing physical and imaginative connections to other locations (e.g. Pollock and Sharp 2007, Sharp 2007, Rogers 2011). As the former NBGW Director, Rosie Plummer, is quoted as saying on the Size of Wales website

Getting close to and touching and smelling a rainforest tree is an experience few will have in their lives and we are sure people will want to come from all over Wales and beyond to share in this astonishing experience. ${ }^{9}$

The flipside of a global imaginary such as this is that, while the Ghost Forest brings another place to people's attention, it is essentially placeless, having been moved nomadically around the world, with no particular roots to the places in which it is exhibited. This corresponds with Leuthold's assessment of art in Western-Enlightenment thought, in which, he contends, art tends to be something that is for "sale or exhibition as a commodity or an independent object that extends beyond a community" (Leuthold 1998, 6). He contrasts this with situations (usually non-Western) where artworks are community-orientated and arise out of the cultural context. His assessment echoes Heidegger's hypothetical question "does not the flourishing of any

\footnotetext{
${ }^{9}$ Available at http://sizeofwales.org.uk/spectacular-ghost-forest-finds-spiritualhome/. Last accessed 23 October 2017.
} 
genuine work of art have its roots in a native soil?" (Heidegger 1966, 47 8), a sentiment which refers back to Schleiermacher's earlier claim that "a work of art ... is really rooted in its own soil, its own environment. It loses its meaning when it is wrenched from this environment and enters into general circulation; it is like something that has been saved from the fire but still has the burn marks upon it" (quoted in Wampole 2016, 141). The metaphors of rootedness and native soils should be treated with considerable caution, given how they can be easily expropriated for nationalistic and racist causes (e.g. Massey 1993, Wampole 2016). Indeed, it seems a rather conservative approach to art when juxtaposed with the more fluid, "cosmopolitan" view outlined above. However, and particularly in relation to socioecological transformation, this topic is worth exploring a little further, particularly given how starkly the Ghost Forest artwork represents an enormous act of up-rooting, and how this contrasts with Emergence's intensely rooted activities.

Emergence demonstrates an approach which perhaps aligns more closely with the idea of an art more "rooted in its own soil". The themes of the walks that Emergence organise are contingent upon place (relative to South Wales); for example, a Peace Walk along Swansea sea front to commemorate the seventy-fifth anniversary of the World War Two bombing of Swansea; another walk, celebrating harvest-time in September 2015, was a pilgrimage to Maen Ceti (Arthur's Stone), an ancient Ne-

olithic burial tomb and capstone on the nearby Gower Peninsula. In what follows, I describe my experience of joining The Walk That Reconnects in 2014, and explain how this experience entailed a different mode of constructing and relating to place than the Ghost Forest's "global" approach.

\subsubsection{The Walk That Reconnects}

The Walk That Reconnects Land Journey was one of Emergence's most ambitious group walks, and was part of Swansea's Love Your Countryside festival in Summer 2014. I joined the company of twenty-two other walkers, ranging in age from twentytwo to seventy, on a four-day walk along the Gower Way in South West Wales. The invitation to join the Land Journey explained that

We will connect with inner intentions and outer landscapes with an awareness of the many feelings, thoughts and emotions that arise out of this rich territory from gratitude and appreciation to pain and grief ... In community we will create the conditions for each of us to move into a more 
expanded 'ecological self', uncovering our essential inter-connectedness with one another and the world.

Walking provides what Ingold and Vergunst $(2008,3)$ call a "tactile, feet-first" means by which do to this a gateway to other ways of engaging with the environments, webs, landscapes and communities within which we move. Abram suggests that "humans are tuned for relationship. The eyes, the skin, the tongue, ears, and nostrils all are gates where our body receives the nourishment of otherness", adding that "direct sensuous reality, in all its more-than-human mystery, remains the sole solid touchstone for an experiential world now inundated with electronically generated vistas and engineered pleasures; only in regular contact with the tangible ground and sky can we learn how to orient and to navigate in the multiple dimensions that now claim us" (Abram 1997, ix-x).

During the Land Journey, it was difficult to escape the immersive experience of walking, sleeping and eating outside for four days and four nights a rare opportunity for anyone who, like me, spends the majority of their days inside buildings. Along the way we braced ourselves along blustery ridges, walked barefoot on sandy beaches, ate blackberries from hedgerows, sat around campfires, and swam in the sea, as well as venturing through industrial sites and town centres. In many ways these sensory experiences had to be wilfully negotiated; walkers had to be open to experiences and attentive to their senses. Indeed, almost all of the walkers, when asked on the first evening why they had decided to come, made some reference to wanting to connect with nature and the outdoors some used the words to "ground", "heal" and "recharge" in nature. As a walker I found I had to "learn to be affected" (Latour 2004a), a matter of creatively learning new skills, techniques and bodily intelligences, which have been highlighted as an important aesthetic dimension of socioecological transformation for broadly (Hawkins 2017, Hawkins et al. 2015b). "The world perceived through the feet" (Ingold 2004) provides opportunities for imagining and experiencing the world otherwise, shaping a relational consciousness which is as much dependent upon the senses as it is on the intellect. Indeed, Ingold (2000) suggests that the better attuned a person is to the rich and varied textures of their surroundings, the better able they will be to find their way about, both literally and figuratively. However, it can also be argued that the value of such encounters with the more-thanhuman world lies as much in the production of an awareness of human limits (of perception and understanding) than it does in producing any kind of instrumental knowledge (e.g. Smith 2011), and thus cultivates a certain humbleness or feeling of smallness. Again, the ways in which Emergence engages with walking as a mode of 
creativity and "art" resonates with a Dionysian emphasis on closeness of encounter, rather than critical distance, and with Dewey's notion of the "arts of living" as activities which stimulate "immediate enhancements of the experience of living" (Dewey 1934, 30). Emergence's emphasis on face-to-face communication can also be thought of in this light, as attempts to facilitate meaningful encounters between people that have a certain intimacy that is not available through digital technologies that prioritise the visual/representational over the oral and which, although powerful in many ways, are abstract and placeless (Abram 1997). For example, the impetus for organising the COP123 events in 2015 emerged from a desire to generate a local response to the United Nations Conference Of Parties (COP) climate talks happening 500 miles away in Paris, and to the global phenomenon of climate change. Smith, speaking at the first event in Swansea, remarked that instead of travelling to Paris for the talks and marches, she felt she wanted to

stay home and to start talking to people locally, and find out what's going on already that I could be part of, or just ... getting more people into the conversation.

Again, although this might sound like a rather trivial response to climate change, the contrast between closeness of experience and critical distance is, I suggest, a key difference between Emergence and the Ghost Forest and the work that each does, particularly in terms of how each constructs geographical imaginaries of place and "topologies" of the environment.

\subsection{2 "Topologies" of environmental art}

Ingold's thoughts on the "topology of environmentalism" (Ingold 2000, 209) are useful here for emphasising how the "art" of the Ghost Forest and Emergence are underpinned by and reproduce very different conceptions of the relationship between humans and environment (fig.5.3). The Ghost Forest is a global project with a global message. It implores its viewers to cast their imaginations to distant places, while simultaneously highlighting just how dominant and destructive a force human activity can be. By contrast, Emergence focuses its attentions on the more immediate lifeworld, encouraging people to familiarise themselves with one another and with their surroundings. Its walking activities help to foster what Ingold (2000) calls a "dwelling perspective", that is, a sense of being in the environment rather than a force upon it. Walking entails perceiving the environment from within, whereas observing an art 


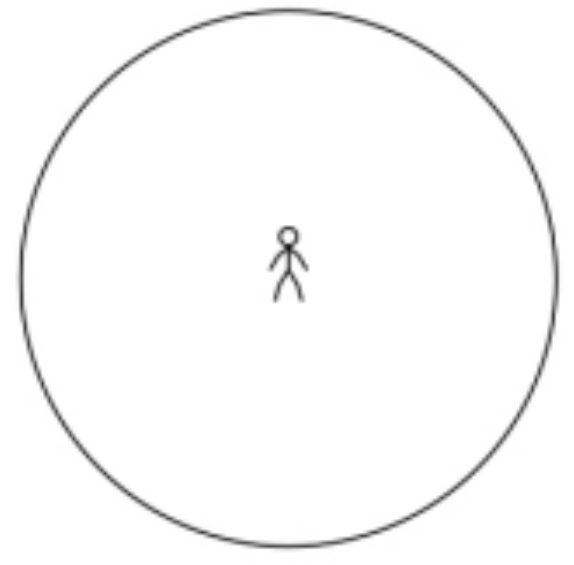

A

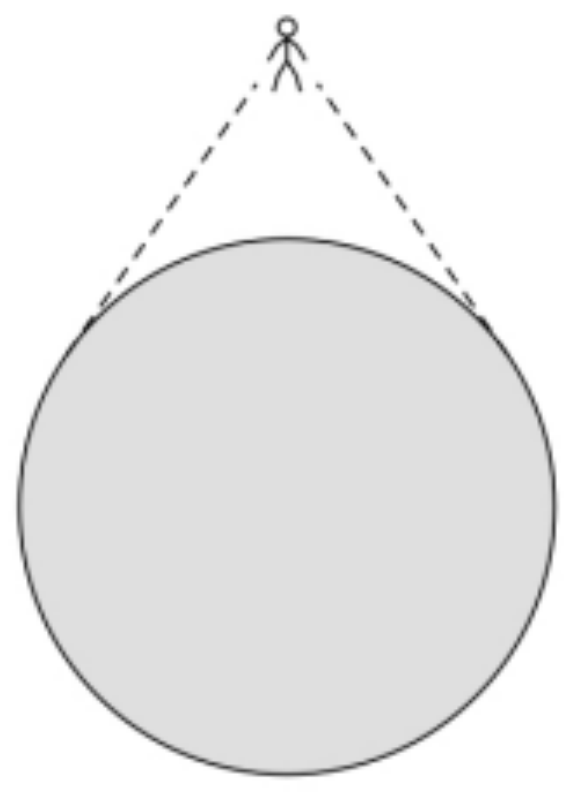

B

Figure 5.3: Two views of the environment: (A) as a lifeworld; (B) as a globe (Ingold 2000, 209)

installation entails perceiving it from without.

The Ghost Forest is an aesthetic experience that in many respects privileges visual perception (it relies upon the size and shape of the trees for its most immediate impact, although touch, as mentioned, is also important). Visual perception, according to Ingold, reinforces a sense of externality of the perceiver, whereas auditory perception (as privileged by Emergence in terms of its emphasis on conversation and on silences (see below) while walking) places the listener at the centre of a sphere a lifeworld. Thus, as Ingold $(2000,211)$ says "whereas we appear to be on the edge of the visual space looking in with the eye, we are always at the centre of auditory space listening out with the ear". And although the "globe" outlook (associated with phrases such as "global environmental change") is fast becoming ubiquitous particularly in relation to sustainability Ingold argues that is a perspective that actually expels humanity from the lifeworld, such that, rather than the environment surrounding us, it appears that it is us who have surrounded it. "Far from reintegrating human society into the world of nature", Ingold says, the idea of a solid globe "marks their final separation" (ibid. 155). Ingold's conclusions on visual perception can, however, be tempered by a more nuanced approach to vision which understands vision as a 
more "enfleshed" (rather than distanced) sense, "relocating it in a desiring body and situating sight amid a 'bouquet' of the senses" (Hawkins 2013, 61). Nonetheless, the concept of dwelling is helpful for thinking through some of the differences between Emergence's and the Ghost Forest's approaches. Ingold's (2000) thoughts on dwelling and the lifeworld draw on Heidegger, who in association with his views on art and rootedness saw the experience of dwelling as one which is necessary for developing an ecological sensibility. By dwelling, he contended, we develop a relational awareness of the "fourfold" earth, sky, mortals and the divine for we are literally enfolded in it. He considered both walking and language to be integral aspects of dwelling, because walking is a means by which to "gather a place together in our phenomenological understanding" (Lack 2014, 54), while language is literally "the housing in which humans dwell" (Padrutt 2009, 17). As such, walking and talking can help us to become more fully aware of our "enfoldedness" within life's webs human and non-human, harmonious or otherwise.

Interestingly, the Ghost Forest installation has undergone somewhat of a transformation in this regard. Prior to their arrival at the NBGW, the trees were displayed on white plinths, held in place by steel cables. Their locations were always urban (London, Copenhagen and Oxford), creating a juxtaposition between the trees' forms and the urban shapes and colours, and at night the trees were lit from below. Framing them in this way created quite a dramatic visual spectacle, in which "nature" is something "over there", the charismatic and exotic rainforest trees elevated on plinths, rather than, for example, the grass protruding between the paving slabs over which the Ghost Forest audiences walk. Palmer told me that this way of displaying the trees

was deliberate [...] I wanted to create an art gallery, elevating the trees on plinths like works of art which are to be revered.

This romanticised ideal of "nature" risks reinforcing a view of nature as something that is essentially separate from humans, as well as dubious socio-political stances which rely on a sense of what is "natural" and what is not (e.g. Morton 2007, Castree 2013a). ${ }^{10}$ However, Palmer and the NBGW staff decided that, at the NBGW (which was to be the Ghost Forest's permanent home), the trees should rest directly on the grass, without plinths and cables. In addition, the NBGW's rural setting means much of the striking juxtaposition is lost and the trees appear more organic in the

\footnotetext{
${ }^{10}$ Emergence's relationship with walking could also be accused of a certain romanticism, given that this way of moving through space in some ways reveres the landscape for its ability to "free the mind" and generate philosophical, intellectual and aesthetic reflexivity (Edensor 2000).
} 
landscape. They are in a relatively discreet location in the Garden, tucked behind the Great Glasshouse, but positioned either side of a footpath leaving the Glasshouse so that visitors can walk between and, if they wish, around them (woodchip has been laid around the trees in order to keep mud at bay).

There are a couple of things to note about this change in display. The first is that it can almost be seen to symbolise a shift between the mode of art which puts "nature" on a pedestal (Morton 2007), and an approach which blurs and dissolves any such distinction between nature/human, art/life. At the NBGW the trees blend in far more with the landscape, they are less imposing the barrier between "artwork" and "viewer" is much less tangible, and the trees themselves will gradually decompose. When I asked Palmer herself about this change, she told me that

the juxtaposition was most potent at the feet of Nelson in Trafalgar Square, and of course it would have been wonderful if they could remain in such a brutal contrast. But it would be naïve to believe such a location is available on a permanent basis.

Instead of creating the sense of an art gallery, as she had done previously, Palmer said that she saw the NBGW as more like the trees' resting place "their Embassy" and permanent "International HQ". The NGBW staff have embraced, and to a large extent, initiated this change in display (the NBGW produced new interpretation to accompany the Ghost Forest), and the staff highlight the importance of the trees becoming part of the landscape. Rob Thomas, Head of Development at the NBGW told me that

not having them on plinths was a conscious decision for us in interpreting the lifecycle, it's important to point [out] that things just as we all do eventually become part of the earth again, which is what these will do over time. Um, so that was a conscious decision. Ah, it would have been an intrusion into the landscape to put them [on plinths]. And it would have made them .. um .. somehow artificial artefacts, I think. For us it was important to have them in connection with the ground.

In fact, some staff members seemed slightly uncomfortable with calling the trees "art" at all, as Bruce Langridge, Head of Interpretation told me

they were on plinths before because they were in urban settings and, um ... an element of an art display as well, which goes with that. So, if they 
were on a plinth, they would look probably more like an artistic thing here. But there's no need there's no practical need here ...

Langridge's comment indicates an assumption that the term "art" only refers to objects which are framed and displayed in particular ways. It is interesting to note that the visitor comments recorded in guest books at the sites where the trees were displayed as though in an art gallery (on plinths and with with lighting) recorded many more references to "beauty" than I observed in comments from visitors to the Ghost Forest at the NBGW. At the NBGW, people tended to comment on the trees' sizes and shapes more than a particular sense of beauty which perhaps says something about how people perceive beauty in particular ways (i.e. if framed and presented as "art"). But in fact, what Bruce Langridge went on to tell me about the NBGW's new hopes for the Ghost Forest chimes far more with the kind of "art" which Emergence, too, seeks to cultivate, that is, an art that is not predicated on critical distance but on a whole host of sensory experiences. He described how he thought that the potential for the Ghost forest to inspire changes in people's ecological sensibilities lay less in a doom-laden message and more in learning

to like or to love something in fact I love wildflowers, and if I take people round on a walk I get them to touch and to smell and so on, cos that works so much better than ... if I was to just give a talk on it it doesn't really do so much it's the same [with the Ghost Forest], you can't particularly smell any more, but just touching and seeing, whatever, you know, that might get through to some

In my observations, visitors often interacted with the trees by touching them, and children especially seemed compelled to run to and climb on them (something that Bruce, and other staff, also mentioned they were pleased to see, albeit reluctant to openly encourage for health and safety reasons). I myself enjoyed making these observations from a position nestled in a comfy spot on the flanks of one of the trees, the Denya.

Accordingly, the NBGW has toned down the "message" of the Ghost Forest. Although staff do still mention an intention to use the Ghost Forest to highlight issues of deforestation, this aspect only appears on one small part of the accompanying interpretation. In my experience, visitors were often not aware of this message at all, and simply walked past the trees, commenting on their size or admiring the shapes of the roots, touching and exploring the feel of the wood, and taking photos. The 
sense of loss and sadness which Palmer had observed in visitors to the Ghost Forest's previous exhibitions is much subtler at the NBGW, and, on the contrary, the NBGW are using the Ghost Forest to create a more "life-orientated" as opposed to "death-orientated" atmosphere. One aspect of this is the connection with the charity the Size of Wales which, as already mentioned, is actively working to prevent deforestation. The other is that the Ghost Forest is positioned so that it overlooks a site where the NBGW is planting a Woods of the World, which consists of tree and shrub species from all over the world. Although this is not widely publicised yet, several staff told me that they liked the fact that they could use the Ghost Forest to highlight something positive that was being done a bit closer to home in terms of reforestation.

The themes I have touched upon in this section highlight an inherent tension between the "sensuous and interpersonal contact with place" and another dimension of intellectual "cosmopolitanism", in which we recognise the reverberations and connections of our own actions with the lives of billions of other beings (Harvey 1993, 15). Emergence and the Ghost Forest appear to intervene at different ends of this "spectrum" Emergence at the level of sensuous and interpersonal contact with place, the Ghost Forest at the level of an intellectual understanding of global interconnectedness. Ultimately, though, this dichotomy is misleading for, to take Massey's notion of place, places are not things but are constellations and processes of relations. Even though the Ghost Forest is, ostensibly, a global project with a global message, it is experienced (especially at the NBGW) on a very personal, sensory level which simultaneously cultivates connections with immediate surroundings and with distant places. Conversely, although Emergence concerns itself with very locally-based activities, the ideas that influence it (the Joanna Macy framework, and some Buddhist philosophies, for example) suggest that it, too, is linked into a global exchange of ideas.

Perhaps, then, it is difficult to talk about any art which is truly "rooted" in a particular place, but the discussion presented here does point to the importance of recognising how aesthetic practices and forms contribute to framing people's place in the world, and how some methods might be more conducive to a dwelling perspective than others. Massey (1994) argues for a "global sense of place", and although it seems intuitive that an intellectual understanding of the world as interrelated might be essential to such a perspective, it must surely be a matter, literally, of sensing, too. Affective understandings and embodied relations between humans and the morethan-human world are central to how we live with, act in, and experience our direct 
surroundings (Smith 2011, Cooke et al. 2016). In the next section, I therefore turn my attention to the matter of more-than-human agency and how Emergence and the Ghost Forest deal with it differently.

\subsection{The art of agency}

The giant tree root has been blessed by a river goddess. Why? It didn't want to be moved

(A sentence from the NBGW's interpretation which accompanies the Ghost Forest's Denya tree)

The different topological imaginaries outlined above also point the way to how Emergence and the Ghost Forest conjure varying ontological landscapes with regard to their treatment of subjects and objects, and the notion of more-than-human agency. The above quote, which goes on to describe how the blessings from a Ghanaian elder "did the trick" and the great Denya "moved peacefully out of the forest" in time to make the last possible sailing from Ghana to London, is somewhat paradoxical in this respect. The phrase "it didn't want to be moved" hints at a notion of more-thanhuman-agency, and yet the Ghost Forest project presents a situation in which the trees' agency is somewhat masked by human agency, both literally and symbolically: the trees are made to "do" (and be) things according to human will; not only are they transported around the world, they are also labelled by the artist as "ambassadors" for the world's forests; they are presented as a metaphor for the loss of the world's lungs; made into aesthetic spectacles by placing them on white plinths. Now at the NBGW, the trees continue to "work" by strengthening the Garden's identity and mission statement. As the then Director, Rosie Plummer, explained to me:

I mean, my ambitions [for the Ghost Forest] would be many and varied, you know it's attractive, it's the biggest environmental art installation, it's important, it's got lots of key messages, it can help drive footfall [...] Number one keep the business going! [And] ... are there conservation, education, inspiration messages, motifs, purposes, we can drive from it?

The trees are, effectively, made to speak in particular ways, according to Palmer's (and now, the NBGW's) particular aesthetic techniques and intentions. Such an approach aligns, one could argue, with an objectifying relationship with the more- 
than-human world. This is reflected in some of the language which accompanies the Ghost Forest. For example in Palmer's statement about the Ghost Forest, she describes how seven indigenous species are represented, "all with a rich and varied ecology and all with diverse uses by man." The NBGW interpretation, too, highlights particular human uses that each species has Wawa wood has been used to make electric guitars; Mahogany bark can be used to treat colds; the Celtis for making coffins. The phrase "we are destroying our natural resources" (which appears in the artist's statement about the Ghost Forest) is one that, in general, we are so used to hearing that it is almost taken for granted. And yet, as the previous chapter explored, the use of the words "our" and "resources" implies a deep assumption about human ownership and control, and about the value of the more-than-human world being predicated on their utility in human life. In addition, this representational mode of making sense, whereby the trees are presented in a particular way and made to represent a particular issue, might also be understood in terms of Heidegger's notion of "technological ways of revealing". Heidegger was concerned with an increasing tendency to view and understand the world solely "in relation to man" as the being who "decide[s] ... how [other] beings appear" (Cooper 2005, 345), and in particular the ways in which the more-than-human world is "revealed" that, is, experienced and interpreted via technologies (tools, machineries, or techniques), rather than being allowed to reveal itself.

A dwelling perspective entails a different kind of perspective on the relationship between humans and more-than-human (and of the agencies of both), because it "stresses the physical, relational, sensual, performative orchestration of body and space/environment" (Jones 2009, 303), and practical experience and knowledge of the world through the body (Cloke and Jones 2001, Franklin 2002, Whatmore and Hinchliffe 2003). A good example of how Emergence's walks encourage such a bodily approach to experience is through its experimentations with silence. During the Land Journey, periods of silence became by popular request an integral feature of the experience, with at least one period of walking in silence, sometimes for up to an hour, each day (fig.5.4). In my conversations with them, walkers often commented on the importance of the silences to their experiences of the walk. The silences afforded opportunities to "tune out" of human noise (the chatter of twenty-two other humans is quite all-encompassing!), and allowed walkers to "tune in" to the more-than-human world.

Sontag (2002) has written about the sensory experience of silence itself. Silence, she observes, is never a genuine emptiness, for it exists in and is perforated by a 


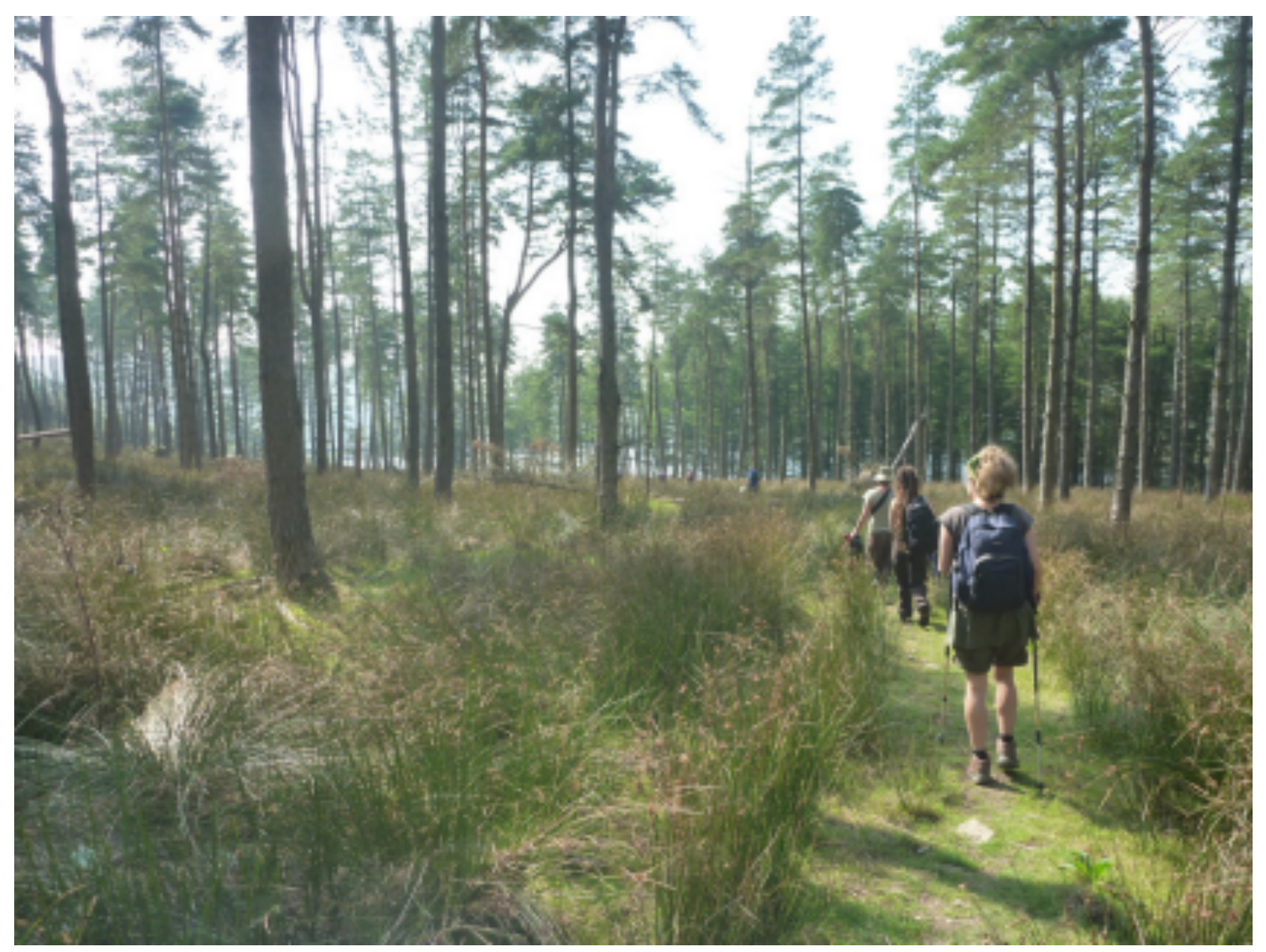

Figure 5.4: A period of walking in silence during the Land Journey, north Gower. (Source: author's own) 
world full of sound. Silence is thus an element in a dialogue and a way of focusing attention outside of one's self in a contemplative manner, such that self-forgetfulness might (if only fleetingly) prompt the dissolution of subject/object binaries in how a person relates to the world. Watts $(2013,32)$ describes how bringing ecological perspectives to life can be a matter of simply listening: "our ears have become dull to the sounds of the land speaking up through our feet ... It is not a question of accessing something ... but simply to listen". This idea of listening to the more-thanhuman world is important, for it is based on an important assumption: that "things" have voices (and agency). Thus, it invokes a rather different ontological, political landscape to one which categorises matter into subjects and objects (e.g. Bennett 2009). For me, the silences on the walk provoked an attunement with the more-thanhuman-world around me. Bawaka Country et al. (2016, 463) suggest that this kind of engagement is what allows someone to "hear the [land's] language and develop an understanding of its unique material presence, its patterns and place/space. In this way, embodied engagement fosters knowing specifically, a form of knowing that is based on a recognition (perhaps conceptual, perhaps sensory) of more-than-human agency". Attunement, here, is not necessarily meant in a romantic or "harmonious" sense, but rather as an alertness and awareness to the sights, sounds, and sensations of the body and of the materials, sensations and beings interacting with it. Importantly, it is not necessarily a tool for revealing more-than-human agency, but rather a means of tuning into more-than-human agency as it reveals itself. Whereas the Ghost Forest (particularly pre-NBGW) imposes a particular "voice" upon the trees in the ways in which they are framed and narrated, Emergence's approach seeks to facilitate ways in which people might become more available to the voices of more-than-human others to hearing the "the language of [the] soils and winds and birds" (Bawaka Country et al. 2016, 464). Again, this corresponds to Ingold's differentiation between whether we understand our geographical self as either within or outside of the lifeworld.

A de-centering of human agency has been identified by many as an essential aspect of socioecological transformation (e.g. Whatmore 2002). Guattari ([1989] 2014) suggests that the articulation of a nascent subjectivity will be key in any attempt to address the socioecological crises of our era, requiring new social practices, new ways of thinking and feeling, and new practices of the self in relation to the other. And yet, it is much less clear how this might be achieved. As Gibson-Graham and Roelvink (2010, 322) ask "how do we get from an abstract ontological revisioning to a glimmer or a whiff of what to do on the ground?" Morton (2007) contends that aesthetics is crucial in this regard, by establishing ways of feeling and perceiving place otherwise, 
and Jackson $(2016,15)$ adds that "an unreconstructed aesthetics will not reveal a reconstructed subject or, necessarily, a reconstructed politics". Aesthetic practices are central to how subjectivities are constructed and performed in everyday life, and, in turn, to possibilities for socioecological transformation, because the relationships of subject/object that are made through art might also map on to, reflect, or influence the perceived relationships between subjects/objects more generally (Miles 2014). The implication is that, in changing how we think about "art", we may, too, be able to change how we think about and behave in the world. This sentiment extends a mandate that seems largely unfulfilled in the context of much well-intentioned environmental and climate art, especially where it relies on representational modes of making sense that implicitly reinforce notions of "nature" as the object (of the artwork), and of the audience as the sensing, feeling but separate subject (Demos 2013). In the following section, I explore this terrain in more detail, focussing on how ideas about environmental art and the artist might be reconceptualised in ways which have more in common with non-Western/pre-Enlightenment approaches to art, and what relevance this has for socioecological transformation.

\subsection{Re-imagining environmental art?}

If aesthetic practices are central to re-imagining subjects and objects (indeed, dissolving that very distinction) when it comes to how humans relate to more-than-human others, then this has implications for how the concepts of "art" and "artist" (and the work that each does) are configured. Environmental art (or eco-art) is a term which emerged in 1960s and which, although not strictly defined, usually refers to installations of one form or another, sometimes fixed and sometimes ephemeral. Although such work is, by definition, "relational" (Bourriaud 1998) because it takes into account the broader social context (e.g. Wallen 2012), much climate and environmental art in contemporary Western contexts maintains an attachment to ideas about creative agency primarily in the hands of particular people and at particular sites. In what follows I explore some of the implications of this, and how it might be re-imagined otherwise. 


\subsubsection{Re-thinking "the artist"}

The leading arts and climate change organisation, Cape Farewell, describes on its website how "Cape Farewell engages and inspires our greatest creative and visionary minds to work with scientists, clean-tech entrepreneurs, sociologists and designers to vision and help create the non-carbon society we must all aspire to." ${ }^{11}$ Other prevalent organisations in the field of art and environment consistently refer to the work that "the arts sector" and "artists" can do with regard to socioecological transformation. ${ }^{12}$ This approach highlights an intriguing mix of values and approaches to art. Such work is socially-concerned and thus does not align with the "art for art's sake" ethos which became prominent from the 19th Century onwards, especially in Europe a movement which insisted on the autonomy and "purity" of art, separate from any social function (see Benjamin [1935] 2008). Simultaneously, however, it reproduces an autonomous approach to art, in that it adheres to ideas about creativity as associated with "lone geniuses" and with great creative achievements. This way of constructing notions of who and what "the artist" is relates to philosophies of mind and knowledge which developed during the Enlightenment and Romantic periods (Leuthold 1998, Glăveanu 2011).

The Ghost Forest appears to fit this model of aesthetics: It is clearly presented as the work of one person, Angela Palmer, and her creative vision and drive to realise the project are central to the story which accompanies the Ghost Forest. The Ghost Forest is consistently referred to as the work of "artist Angela Palmer", and on the Ghost Forest website it is written that the Ghost Forest is "Angela's most ambitious and logistically challenging work yet". ${ }^{13}$ Not only is the Ghost Forest celebrated as the unique and impressive achievement of one artist, but it is also frequently recognised and related to as such by Ghost Forest visitors. For example comments left in the visitor books frequently identified and congratulated the artist:

Great exhibition, wonderful idea Angela.

Angela, a testament to what one person can do. Ten magnificent specimens of Mother Nature.

\footnotetext{
${ }^{11}$ Available at http://www. capefarewell.com/. Last accessed 23 October 2017.

${ }^{12}$ For example, see https://artistsandclimatechange.com/ and https://www. juliesbicycle.com/. Last accessed 23 October 2017.

${ }^{13}$ The Ghost Forest website has since been taken down, but the same statement can be found at http://artlystlondon.blogspot.co.uk/2009/11/angela-palmer-ghost-forestinstallation.html. Last accessed 23 October 2017.
} 
Let us be good stewards of this planet. Angela deserves an MBE for drawing attention again to the corruption issue.

An amazing exhibition. Definitely a pioneer. Great that you did this.

It is amazing what a person with a clear goal can do bringing these trees into London!

Angela, how important it is to have artists in a changing and fragile world. Sometimes only you can find ways of communicating with people's souls. Congratulations and 'Obrigada'!

Palmer herself also highlights how she perceives her role as a relatively autonomous one, despite the social and environmental content (and context) of the Ghost Forest work. She told me, in relation to the Ghost Forest and other work which has included diverse subjects, from MRI scans of the human body, to Formula 1 engines, that

I am first and foremost an artist; I am not an eco-warrior whose work is about creating art with an environmental message. I don't foresee any restrictions to my areas of exploration.

She is more inclined, as she says in her artist's statement on the Ghost Forest website, to see her projects as a "collision between art and science." ${ }^{14}$ Her comments give an insight into how her own conceptions of art and artistry are framed by a WesternEnlightenment perspective which emphasises the creative autonomy of the artist, and the perceived separation (as in the Cape Farewell quotation, above) of the domains of "art" and "science". Critics and analysts of environmental art perpetuate a sense in which creative agency (and responsibility) rests with "artists", for example Brady (1998, 142), in relation to aesthetic appreciation of nature, comments that "much depends on the ability of the artist to create an engaging and imaginative work of art."

In contrast to this conception of the artist, Emergence's website states that

Emergence is not about bringing 'artists' together with 'ecologically minded people', 'economists', 'activists' or 'scientists'. This approach embeds the reductionist thinking we are attempting to move away from.

\footnotetext{
${ }^{14}$ The Ghost Forest website has since been taken down, but some context to Plamer's work can be found at https://www.inverse.com/article/4746-modern-artist-angela-palmer-onsculpting-from-science. Last accessed 23 October 2017.
} 
Emergence's approach does not, therefore, single out "the artist" as separate or distinct from the rest of society: from its perspective, each walker and talker attending Emergence events is an artist. This marks an important departure from more autonomous forms of "environmental art" which as Cape Farewell describes depend upon the leadership of particular visionary individuals. In place of this model, Emergence suggest that there is

a need for experimentation, spontaneity and diversity which sees us all as 'co-creators' of the future rather than as 'co-dependents' reliant on specialist knowledge or heroic leadership

In conversation, Emergence's founder, Fern Smith, told me that she was concerned about the "active artist, passive viewer" dynamic entailed in much environmentallyconcerned work. Films, for example, are becoming a popular way of conveying quite hard-hitting environmental messages (recent examples include, Cowspiracy: The Sustainability Secret (2014), and Al Gore's An Inconvenient Sequel (2017)), but, as Benjamin ([1935] 2008) observes, such art does not usually require much effort or concentration on the part of the viewer it is merely "absorbed" by them, keeping them distracted and absent-minded. Films are an extreme example of such a dynamic, and certainly participatory environmental art projects, such as those facilitated by Cape Farewell, are likely to inspire more active engagement. Nonetheless, if creative agency and leadership are located primarily with the artist(s) in these cases, then the "active artist, passive viewer" dynamic is still of some relevance to understanding how (and if) such approaches work.

\subsubsection{Re-defining "art"}

In its desire to reconfigure this dynamic, Emergence draws on philosophies and approaches to art which are by no means new but seem to have found little traction in recent approaches to art and environment: from the Indian philosopher, Ananda Coomaraswamy's, oft quoted insistence that "an artist is not a special kind of person, but every person is a special kind of artist" (Coomaraswamy 1935), to the German sculptor and art theorist, Joseph Beuys', conviction that "every human being is an artist . . . the essence of man [sic] is captured in the description 'artist'" (Beuys quoted in Gandy 1997, 639). It is in this spirit that Neal writes that the "transformation of society could be regarded as one great work of art to which each individual person contributes creatively" (Neal 2015, 3). Beuys' theory of social sculpture in- 
vited people to understand themselves as an important part of a whole, not only an individual, and he was a strong proponent of the idea that every decision is an opportunity to make or contribute to a work of art that, in the end, is society (Weintraub et al. 1996). A useful illustration of such an approach is Mierle Laderman Ukeles's (1969) Manifesto for Maintenance Art, a proposal which posits that maintenance in everyday life even the most mundane tasks like household cleaning is art, for these activities are directed towards what Ukeles calls the Life Instinct (a concern with relationality and the perpetuation and maintenance of species), as opposed to the Death Instinct of "Avant-Garde" art which emphasises individuality and autonomy (Ukeles 1969). It is in this sense that Lack (2014), following Heidegger, likens the role of the "artist" to that of a gardener, and "art" is something which assists in maintaining life's emergence.

Dewey wrote in Art and Experience that "we must recover the continuity of aesthetic experience with the normal processes of living ... If art were understood differently by the public, art would gain in public esteem and have wider appeal (Dewey 1934, 3). Dewey's (1934) work detailed a view on the value of aesthetic experience which extended from his work on pragmatism a school of thought which views knowledge as arising from active participation and interaction of humans with their surroundings, and which rejected dualistic approaches to philosophy or, for that matter, to creative work. His approach countered the influential Kantian conception of aesthetics which entails a certain disconnection from direct, sensible, experience, and which has been highly influential on approaches to art and aesthetics in the context of post-Enlightenment, Euro-American culture (Jackson 2016). As Miles (2010, 19) contends, "art inevitably distances whatever problems it addresses the real is always mediated and distanced in culture", and yet, I think it is possible to question such inevitability. Like Dewey, Emergence's interest is in the experiences of everyday life, and the realisation that daily activities often involve perception and care, creativity and artistry (Joseph Beuys' example was that of peeling a potato). Marcuse's vision of art expresses similar hopes for social transformation through the merging of art and life, "in which a new sensibility and aesthetic values would be part of the construction of emancipated individuals in a nonrepressive society" (Marcuse 2007, 27). Emergence conceptualises art in a way that questions the inevitability of the distance between text and world proposed by Miles. In fact, by its own admission, Emergence struggles to define what it is that it "does" exactly, but a phrase that continually surfaces is "artful living within ecological limits". As Fern Smith explained at one event in 2015: 
... we keep coming back to this phrase 'the art of living'. So, art is not a separate activity from life, but we actually join the two together. So, there is no division between art and life. And really that's what we're trying to do with Emergence, which is really ... a creative experiment. Where we ask people, invite people, to come and join us, in those creative experiments.

Such an approach attempts to avoid the "passive viewer" problem by abolishing notions of viewer or audience altogether. Instead, each individual is recognised as a creative contributor, whether or not they identify themselves as an "artist". Kagen describes how we might therefore think of art "as a verb", a stimulation of one's imaginative capacities and an ability to unearth "one's repressed intuitions and knowledge, kept buried at a subconscious level" (Kagan 2014, 32). Essentially, as Kagan describes, art-as-verb is about

Empowering oneself as a change-agent in society, changing one's self-image and perceived capacities to exercise influence and make a change, reducing inhibitions and healing from apathy that is, reducing fear and stress induced by the social context, and catalysing personal and collective motivations and commitments for change. (ibid.)

A key underlying motivation for Emergence's work is a sense that in many instances people have lost touch with their innate capacity for imagination and creativity, and that this has profound implications for individual and collective capacities for transformation. Ian Rees, founder of the Annwn Foundation for the study and practice of Deep Imagination, speaking at an Emergence event in 2015 (a group walk and meal celebrating harvest time), emphasised this, saying

it's my experience ... that all people are profoundly creative. It's also my experience that most of us are to differing degrees ... stunted in our creativity, blocked in our creativity ... and not aware of its potential.

At another Emergence event, a Peacewalk in 2015 in Swansea, the peace and environmental activist, Satish Kumar, told attendees that

We are all artists. But our art is dormant, our art is .. suppressed. Our art is just lying, buried, under the [...] timetables and plans and jobs and nine to five routines and computers and what not. We are copying, copying, copying, never have our time to use our imagination and our 
creativity and our spontaneity and our improvisation [...] art is not a separate activity, it's a part of everyday life ...

It is in this spirit that Emergence approaches talking and walking as "artful" activities. As Fern Smith explained to the participants of COP123, she became disillusioned with her work as a theatre practitioner and instead became interested in conversation as an artform, and in its transformational potential:

I feel that everything that really begins and has momentum and has ... a kind of enduring power and the possibility to change starts with connection, relationship and ... conversation and dialogue [...] Actually [...] that is often the most creative thing that we can do, as human beings.

For Emergence, dialogue requires imagination and creativity, flexibility and improvisation, and it has the potential for bringing alternative perspectives and understandings into view. The idea that dialogue can have a powerful formative affect on people's imagination, understanding, and education is not new. The Brazilian philosopher and educator, Paulo Freire, advocated dialogue not only as a pedagogical approach, but also as a process that involves respecting people as active and creative subjects (Shor and Freire 1987). The physicist, David Bohm an influence on Fern Smith's approach to Emergence's work developed a theory of dialogue (which became known as Bohmian Dialogue) that advocates practices of deep listening and open communication. Bohm's belief was that this kind of dialogue was what was needed in order for people to live alongside one another in an increasingly complex and interdependent world indeed, he drew on his physicist's understanding of a participatory universe where meaning is constantly evolving and tried to find ways to apply this understanding to social life (Bohm 1996). Emergence's desire to create spaces for conversation resonates with Kester's (2004) notion of "dialogical aesthetics", whereby open-ended, dialogical interaction is itself understood as the "work" of art. As Kester $(2004,8)$ contends, dialogue is "an active, generative process that can help us to speak and imagine beyond the limits of fixed identities, official discourse, and the perceived inevitability of partisan political conflict." ${ }^{15}$

Emergence's approach to dialogue also resonates with some oral traditions, where various customs are often used to help foster "the use of utterance as an aesthetic

\footnotetext{
${ }^{15}$ One example of a similar approach being used in relation to climate change can be found in the Climate Conversations initiative (https://climateconversations.org.uk/), but this differs from Emergence in that it appears to be more "outcome-orientated" (in contrast to Emergence's focus on meaningful, intimate encounters between people), and it does not conceptualise conversation as a form of art in and of itself.
} 
means of expression" (Zirimu quoted in Ignatov 2016, 77). Ignatov (2016, 86), for example, draws on his experiences with Ghanaian cultures of orature to show how "some traditions of political thinking make an appearance only when they are told or performed". Recognising this power, as Kanngieser (2012) urges, requires convivial and caring practices of speaking and listening, a sentiment which echoes Guattari's ([1989] 2014, 29) observation that "it is not only species that are becoming extinct but also the words, phrases and gestures of human solidarity". The transformative potential of conversations may go beyond the level of shared cognitive understanding and take a more embodied form, too. For example, Le Guin (2004) describes how speech is, fundamentally, a physical, bodily process and thus listening is not so much a reaction or a response, but a process of connection. By listening, she suggests, we synchronise with the people we're with, physically getting in time and tune with them. Mutual communication between speakers and listeners can therefore be a powerful experience: words are events that transform both speaker and hearer, not only in the sense of the words' content or communication of meaning, but also in the sense of something else "unsaid" that is transmitted. ${ }^{16}$ Seen from this perspective, conversation can be thought of as a kind of "sympoiesis" (Haraway 2016, 58). That is to say, in connecting through voice indeed just the very act of being together as bodies in a space (McNally 2015) there is a generative effect of making-together, and of bringing new perspectives, attunements and worlds into being. The concept of communitas is useful here. Communitas describes how people experience liminality together, sharing a common experience of solidarity such that one's own sense of self and social difference is interrupted: "a dizziness, a syncope, a spasm in the continuity of the subject" (Esposito 1998, 7). This transient personal experience can be a site of personal and social transformation, and has a sacred, spiritual quality (Turner 1969).

Similarly, walking can be considered an "artful" activity in that, as a "technique of the body", it is a creative and sensory experience, central to how people think, act, and dwell (Ingold and Vergunst 2008, 1). Solnit describes how "walking shares with making and working that crucial element of engagement of the body and the mind with the world, of knowing the world through the body and the body through the world" (quoted in Vaughan 2009, 316). Her connection between making, knowing, and walking is interesting, for it alludes to the etymology of "art", from the Latin "ars", meaning, essentially, a "skill" which is learned and practised (Gaztambide-

\footnotetext{
${ }^{16}$ While Le Guin highlights the potentially beneficial power of words in this instance, it is also worth bearing in mind the relationship between language and control, whereby particular structures of language compel obedience and hierarchy, rather than transformation or dissent (e.g. Deleuze and Guattari (1987) on order-words and passwords).
} 
Fernández 2014). This way of conceptualising the "art" of walking is subtly different to other forms of artistic engagements with walking which tend to focus on particular acts of walking as self-contained artworks or performances, for example Francis Alÿs's urban walks (see Pinder 2011), or the walks and installations of artist Richard Long (see Vaughan 2009). Walking has also been considered as a "wilful and artful" act when undertaken as mass performances of political resistance (Lorimer 2011, 24), but, in my experience, Emergence's walks are less concerned with the subversive potential of walking as an "untethered nomadism" (Pinder 2011, 687), than they are about cultivating a dwelling perspective through walking (see, for example, Edensor 2000, Ingold 2004, Ingold and Vergunst 2008).

In summary, there are important differences between this kind of embodied "artfulness", and the kind of art that is associated with particular objects or spectacles, and with novelty, innovation, and critical acclaim. Chief among these differences is that the latter approach requires an object (the artwork) and a subject (the viewer), and an act of judgement and interpretation by which the viewer comes to understand the artwork's "meaning". Indeed, as already mentioned, the transformative potential of environmental art is often deemed to lie in the artist's ability to effectively convey particular environmental messages or emotions (e.g. Brady 1998, Miles 2010). Such an approach may often be effective at conveying such messages, but, arguably, does little to foster nascent, more relational, subjectivities (as deemed necessary for socioecological transformation) because those works themselves are predicated on assumptions about subjects and objects. If this is the case, then the potential for socioecological transformation would appear rather superficial. Emergence's approach to art offers some insight into how art can be conceived of and practised in rather different ways, which might entail more profound socioecological transformation. In the final section, I try to draw out the link between this reconceptualising of art and its socioecological potential more explicitly, by turning to some conceptions of art which challenge the Euro-American-Enlightenment model of aesthetics. I also point to some potential tensions inherent to such a reimagining of art, particularly in the context of Western societies.

\subsubsection{Geopoetics and indigenous ecopoetics}

Dewey $(1934,147)$ articulated a notion in which art is rooted "deep in the world itself", and that artistry is but an expression of the rhythms of the earth (for example, 
day and night) changes and repetitions which, for Dewey, are deep-seated in the human subconscious. He writes that "underneath the rhythm of every art and of every work of art there lies, as a substratum in the depths of the subconsciousness, the basic pattern of the relations of the live creature to his environment" (Dewey 1934, 150). Grosz (2008b) develops this theme in an essay Chaos, Territory, Art (although with recourse to Deleuze rather than Dewey), in which she details a philosophy of art as the very manifestation of Earth's primordial energy, a force which is by its very nature excessive. Art is the result of life's unstoppable impulse for more to create and recreate and is simultaneously an enhancement of the feeling of life and a stimulant to it, "songlines" (Grosz 2008b, 50, drawing on an aboriginal concept) that join a people to the land and land to a people. Echoes with Marcuse's conception of Eros reverberate, as they do with Heidegger's notion of physis, which relates creative impulse to the self-emergence of life (see Bolt 2010). In the emerging field of geopoetics, similar themes are developed. Geopoetics (meaning, literally, "earthmaking"), although often used to refer to objects recognisable as "poems" (for example the work of geographer-poets, and research methods incorporating poetry), can also be understood more broadly, as a kind of geophilosophy (Magrane 2015). Following White (1992), geopoetics-as-geophilosophy entails a geo-cosmological sensibility which helps us to think past the dualisms of Western aesthetics, "past questions of subjectivity objectivity, art science, nomothetic ideographic, and imagine and enact other ways of inhabiting the world" (Magrane 2015, 97-98). Magrane (2015, 94) describes how geopoetics-as-geophilosophy "employs the widest conception of a poem, where climate change and the Anthropocene themselves [...] are large-scale geopoetics" and in need of geopoetical responses; practices of radical experimentation in making new worlds. According to White $(1992,172)$, what geopoetics tries to delineate is "a field of presence and activity which has poïetic characteristics, but which has little in common with what is habitually known as "poetry"'. According to this conception, artworks can be thought of not only as "discursive", but also as material processes, part of the fabric of everyday life. Similarly, anything "cultural" must be understood as a part of the ecosystem, not as a reflection upon it (Smith 2011).

This, too, is the direction in which recent work on creativity (e.g. Hallam and Ingold 2007, Edensor et al. 2009, Hawkins 2017) travels: towards an understanding of creativity as a diverse concept, an improvisational quality that encompasses a myriad of daily practices through which the world comes into being, and not only as a singular quality emanating from any single, gifted, human. In this expanded sense, creativity is something 
that living beings undergo as they make their ways through the world ... this process is going on all the time in the circulations and fluxes of material that surrounds us and indeed of which we are all made ... (Hallam and Ingold 2007, 2)

Hawkins (2017) contends that such practices potentially enable collective imaginings of new futures, and the remaking of political, social, and economic spaces, but she acknowledges that the dynamics of how such creativities make worlds remains to be explored in more detail. Indeed, although research on the relationship between art and environment is increasingly challenging a dualistic, Euro-Enlightenment conception of aesthetics (e.g. Dixon et al. 2012, Hawkins and Straughan 2015, Hawkins 2017, Williams 2016), it would seem that, more generally, aesthetic assumptions about autonomy and critical distance (Ingram 2014) remain highly influential (although, see Neal 2015 for examples of art/environment projects which counter this). However, this way of approaching aesthetics represents a relatively thin slice of history, and from the perspective of particular societies (namely, Western ones). The emergent, geopoetic approach to art and creativity outlined above, by contrast, is by no means new it is possible to find iterations of geopoetic sensibilities in many approaches to art and creativity beyond and before the context of Euro-Enlightenment aesthetics. For example, Leuthold (1998) notes that, in many indigenous cultures, artistic and creative practices are integrated with community and daily life (artists are not singled out as above or different to the rest of society), and are directed towards the maintenance of social and ecological functions.

This is a topic which, I am aware, risks slipping into an unhelpful discourse of simplistic "non-western/western, indigenous/colonial, oral/scriptural divides" (Eshun and Madge 2012, 1396; see also Radcliffe 2017b). Nonetheless, a growing body of literature from indigenous scholars indicates that indigenous knowledges and creative practices often share a perspective of reciprocity and responsibility towards socioecological assemblages which pre-date and preempt the "ontological turn" towards posthumanism and interdependence. Some contributions in this vein include Cajete (2000), Sheridan and Longboat (2006), Sundberg (2014), Watts (2013), BallengeeMorris (2008), Bawaka Country et al. (2016), Todd (2016) and many more. Magrane (2017) makes a direct link between geopoetics and indigenous/traditional ecological knowledge practices, noting that the latter present possibilities for re-thinking the human, and re-thinking relations with the land outside of Euro-Enlightenment predicates. He cites Perez's description of an "indigenous ecopoetics" which "re-connects people to the sacredness of the earth, honours the earth as an ancestor, protests 
against further environmental degradation, and insists that land (and literary representations of land) are sites of healing, belonging, resistance, and mutual care" (Magrane 2017, 137). Such approaches also seem to resonate with what Marcuse and Heidegger try to describe in their ideas about Eros and Physis (respectively). For example, Tewa Indian scholar, Cajete $(2000,75)$, describes how his own indigenous worldview is inherently spiritual in that "everything is considered to be 'alive' or animated and imbued with 'spirit' or energy". Similarly, Hall (2014), in her account of Haudenosaunee culture in the garden which she inherited from her mother, describes how "there is a daily and material quality to the work of making, appreciating, giving thanks to, and finally letting go of, the beauty in the world's cycles [...] [I]f an Indigenous aesthetic practice is one that is embodied and rooted in spirit, then burying my hands and feet in soil and breathing the air is how I express and recreate beauty" (Hall 2014, 283).

It is precisely a dissolution of the distinction between artist/audience, text/world, subject/object which, Mignolo and Vásquez (2013) contend, distinguish decolonial approaches to art and creativity (i.e. those which challenge the hegemony of EuroEnlightenment aesthetic practices). Indeed, Mignolo and Vásquez (2013) put forward the concept of decolonial aestheSis [sic] to counter aesthetics which, they argue, is a philosophical theory of beauty and the sublime, based on a Kantian separation of subjects from objects. The ontological foundation of aesthetics is, accordingly, insufficient for provoking truly radical transformations in minds, politics and practices, as it reproduces the conceptual frameworks which "underpin legacies of ongoing human and ecological harm", that is, a perceived separation of nature from culture (Jackson 2016, 19; see also Gaztambide-Fernández 2014). In contrast to an active/passive notion of artwork whereby the artist seeks to "educate" or "awaken" the audience with regard to environmental issues (Kester 2004, xvi), Jackson $(2016,16)$ contends that decolonial approaches seek "to read creative expression through different predicates", including pluriversal approaches to creativity which encompass aspects of daily life and which themselves perform and produce socioecological realities (Leuthold 1998, De Certeau 2011). In some respects, this links to Benjamin's ([1935] 2008) earlier claim that, in the context of modern/industrial societies, art's ritualistic basis has been in decline, and its social function replaced with the doctrine of "art for art's sake". Similarly, Leuthold (1998) suggests that so-called "sacred art" (which entails elements of spirituality and the notion of sacred nature), has been largely out of favour since the mid-nineteenth century in the West, but remains a central pillar of many decolonial approaches to art and creativity. 
Emergence's approach is interesting because its commitment to "artful living within ecological limits" resonates a great deal with decolonial approaches, in that it is not necessarily the content of its work which aims at cultivating some kind of ecological effect (as with the Ghost Forest's environmental message), but the form that its activities take namely, the seemingly everyday, mundane activities of walking and talking. Indeed, walking and talking have been identified as important aspects of some pluriversal creativities. For example, Bird Rose $(2013,6)$ writes that her experiences with Australian Aboriginal societies have deepened her understanding that "life emerges from dialogue amongst persons; it depends on relationality, interdependence, and mutual flourishing". Similarly, Sundberg (2014) observes how, for the Zapatistas in Mexico, the daily practice of walking brings into being important social and ecological connections and interactions; the dialogic politics of walking, talking, listening, and reflecting is how social change occurs and worlds are brought into being. Likewise, for Native American Navajo, walking is a mythic act, an important event in and of itself (Worth and Adair 1972) while, for the Dene people of Northwest Canada, walking is a way of binding narratives to personal knowledge (Legat 2008). Ecologically, these approaches to creative practice are important because they emphasise interaction with, rather than acting upon, others (Horton and Berlo 2013).

Given these resonances, it is perhaps not surprising that Emergence turns to some indigenous creative practices for inspiration, incorporating many non-Western and pre-Enlightenment ideas and traditions into its philosophy and events. For example, the Council method, which Emergence uses to facilitate dialogue (see chapter 3), is a technique that forms the basis of systems for negotiation and reconciliation in many indigenous societies, past and present (Zimmerman and Coyle 1996). Emergence's engagement with walking as an artful practice also draws inspiration from a wide range of indigenous and spiritual/ritual contexts, including pilgrimages, medicine walks, and vision quests, which utilise walking as a way of binding social narratives to personal knowledges and landscapes (Ingold and Vergunst 2008). A more specific example includes the reading of a Native American Mohawk gratitude prayer during Emergence's four-day Land Journey walk (fig. 5.5). At other times, Emergence celebrates the Gaelic tradition of Imbolc, which marks the beginning of Spring, as a means of expressing gratitude to the more-than-human world. Occasionally, Emergence engages with Welsh ancestral storytelling via the Mabinogion, prose stories compiled in 12th 13th centuries (but which derive from earlier oral traditions). For example, in September 2015, Emergence organised a walk on the Gower Peninsula to 


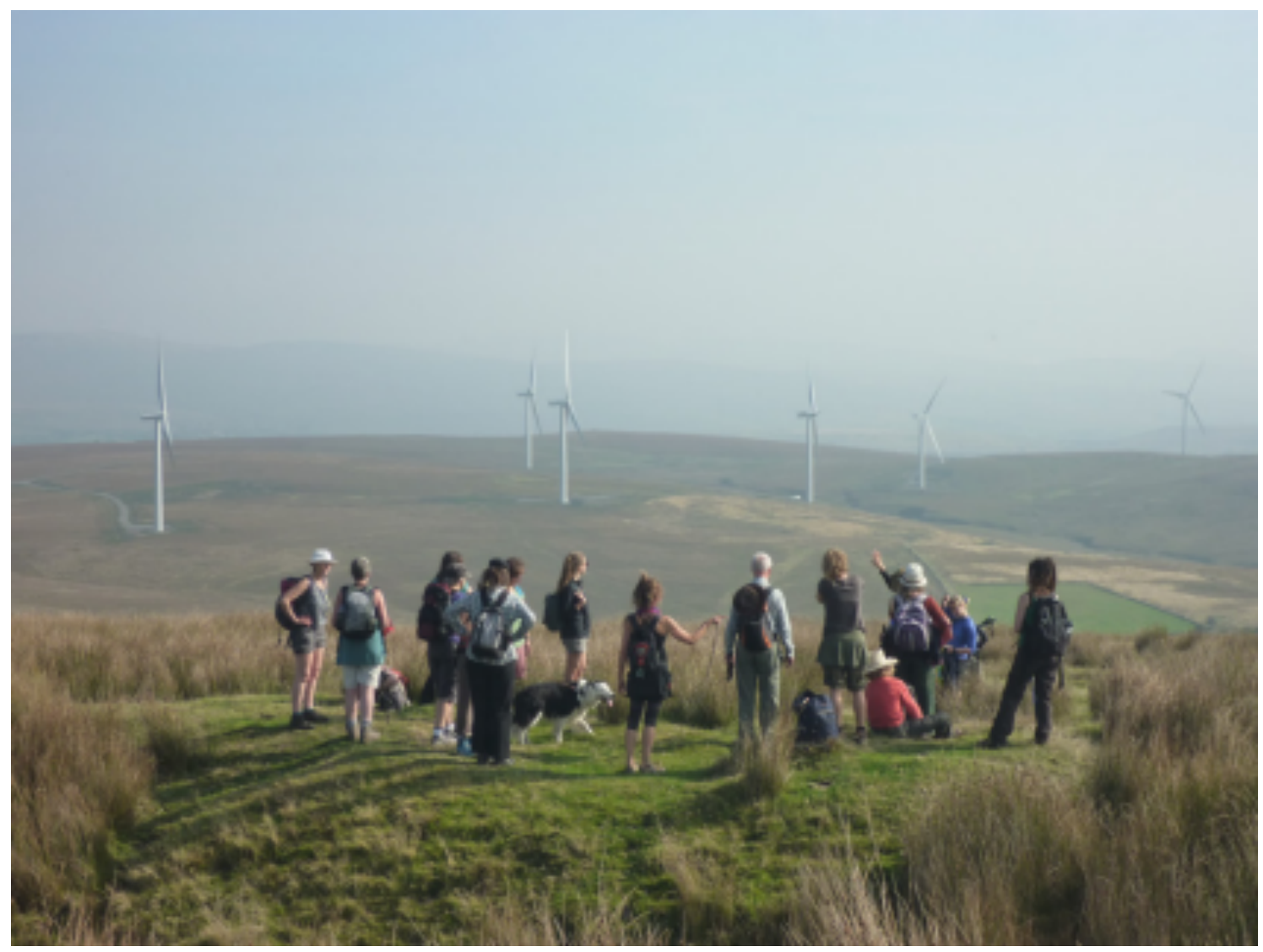

Figure 5.5: Walkers on Emergence's Walk That Reconnect Land Journey in 2014 listen to a reading of a Mohawk gratitude prayer before setting off across the Mawr, north Gower.

celebrate harvest time (itself an expression of gratitude to the seasons) that incorporated inspiration from the Mabinogion tales. The storyteller at the event explained that the purpose of recounting such stories is not to recreate an imagined past but is rather to explore

how our imagination can become empowered to create the new. And one of the really interesting things about the Mabinogion is it draws on very ancient roots, created in connection with the dilemmas of its time

As Jackson (2014) points out, indigenous ontologies are increasingly being invoked in the quest for wiser ways of dwelling, as relational means of understanding the cosmos, of overcoming nature-culture dualisms and, as the quote above suggests, of connecting with the dilemmas of these times. And yet, indigenous peoples are often at the violent edge of ecological crises, and are dealing with ongoing colonial oppression (whether in the form of land rights, or as is predominantly the case in Wales the suppression of culture and language). Given this, there are some questions to be asked regarding whether Emergence's approach to socioecological 
transformation, which appears to align with decolonial creativities by drawing on indigenous practices to nurture ecological sensibilities, is appropriate, and whether it is commensurable with actual decolonisation (Tuck and Yang 2012). In what follows, I turn my attention to this issue because, in negotiating the differences between the Ghost Forest and Emergence, this potential incommensurability remains a crucial sticking point with regards to any transition towards more pluriversal creativities in the context of Western societies.

\subsubsection{Incommensurability}

My own feelings about Emergence's engagement with indigenous ecopoetic practices are mixed. On one hand it seems to me that Emergence's efforts are entirely wellintentioned (see De Leeuw et al. 2013). It shares with many decolonial and environmental efforts a desire to reassert alternative ways to dwell on earth and, indeed, I see it turning to the powerful indigenous movements across the Atlantic as inspiration to (re)discover some indigenous ecopoetical roots in Britain. Moreover, its practices are motivated more by a desire to (re)discover some deep, creative roots ("everybody is an artist") than by a desire to assume the identity of a particular culture, past or present. Its approach resonates with a feeling that

the places of possibility within ourselves are dark because they are ancient and hidden; they have survived and grown strong through darkness. Within these deep places, each one of us holds an incredible reserve of creativity and power, of unexamined and unrecorded emotion and feeling (Lorde quoted in Tuck and Yang 2012, 20).

On the other hand, Emergence's activities could, at worst, amount to appropriation or as "settler moves to innocence" which only focus on decolonising the mind rather than the task of "relinquishing stolen land" (Tuck and Yang 2012, 1). This is most clearly an issue in relation to Emergence's engagement with Native American indigenous practices (such as the Mohawk gratitude prayer), but is also contentious in relation to its evocation of Welsh indigenous practices given that Emergence is directed and participated in by people (including myself) who could be considered "settlers" in Wales. Additional concerns include the absorption of indigenous ecopoetics by the "culture industry", and the possibility that indigenous/subaltern knowledges are in fact merely aestheticised as "geopoetics", subsequently preventing them from being debated in political arenas (Last 2017, 162). There is also a risk of "cherry picking" 
environmental knowledges in indigenous practices, while ignoring other important aspects (Radcliffe 2017b). For example, although Emergence has hosted bilingual events in the past, and it endeavours to translate information about its events into Welsh, on the whole it is a project conducted in English. What problems does this raise, when one considers that the very notion of Welsh indigeneity is so bound up with the Welsh language (and that the legacy of English colonialism in Wales is felt most keenly in the suppression of the Welsh language)? Can the meaning of the Mabinogion tales even be conveyed in English? (Tellingly, the Welsh philosopher J. R. Jones coined the expression "cydymdreiddiad iaith a thir", which, approximately translated into English, means "the co-infiltration of language and landscape"). These are questions that hang in the air, and are certainly not applicable only to Emergence. Conversely, Emergence's interest in indigenous Welsh traditions could be indicative of an "anxiety of settler unbelonging", that is, an effort to adopt the love of the land in order to belong to the land (Tuck and Yang 2012, 15). ${ }^{17}$ Either way, even if the adoption of indigenous ecopoetical practices helps to foster pluriversal creativities, and engender alternative ways of thinking and feeling in relation to one another and to the more-than-human world (as I have suggested in this chapter), it seems that such moves will be incommensurable with actual decolonisation as long as they are not accompanied by a relinquishing of colonial control over land, or, in the Welsh context, parity of the Welsh language.

Where does this leave Emergence's practices and its quest for artful, wiser ways of living? My analysis in this chapter suggests that its geopoetic/ecopoetic practices hold promise in terms of socioecological transformation, particularly in the ways that such approaches challenge a Euro-enlightenment mode of aesthetics that privileges distance and autonomy. Its practices and approach to art might be read as either a "revival" of (or nostalgia for?) indigenous, pre-modern approaches to creativity, or as part of a cultural vanguard experimenting with new aesthetic sensibilities that fit with an "ontological turn" towards posthumanism, interdependence, and relationality. Either way, such developments are complex, and necessitate questions of "where" the geopoetics that might potentially feed this transformation come from (Last 2017), and what and who is included or left out of the script (Eshun and Madge 2012).

At the outset of this chapter I noted that art and aesthetics are increasingly being invoked in the context of the Anthropocene. I want to conclude this section, then,

\footnotetext{
${ }^{17}$ The Welsh word, hiraeth, roughly translates to mean an affirmative sadness, a longing and feeling of belonging to a place - not in a nationalistic sense but in the sense of a fierce and passionate connection with the Welsh landscape. See http://www.newstatesman.com/politics/2007/04/ welsh-language-wales-england (last accessed 26 July 2017) for an interesting perspective.
} 
by drawing out one possibility for how Emergence's practices "fit" in the intersection between the Anthropocene and decolonisation both of which are intimately linked (Last 2015) and pose enormous social and ecological challenges in the 21st Century. My suggestion is that Emergence's artful activities go some way towards unsettling the somewhat colonial register of the "Anthropocene" itself. The Anthropocene concept is contentious for the ways in which it homogenises and flattens "the anthropos", privileges western scientific ways of knowing, seemingly re-asserts anthropocentric ideas, and prompts a binary position of either hope or despair (e.g. Crist 2013, Malm and Hornborg 2014, Haraway 2016). It is argued that none of these positions are adequate for tackling the difficult, complex, heterogeneous and messy work of socioecological transformation. For example, Haraway contends that there is an urgent need to try to "stay with the trouble" rather than jump to conclusions, adding that "we need stories (and theories) that are just big enough to gather up the complexities and keep the edges open and greedy for surprising new and old connections" (Haraway 2015, 160). Interestingly, this insight is one which she credits learning from a book entitled Returns: Becoming Indigenous in the Twenty-First Century (Clifford 2013). Emergence's approach, as I have described in this chapter, is one which in my experience tries to find ways to live with trouble, complexity, and vulnerability. It does not seek to jump to positions of hope, despair, or solutions, but rather tries, through walking and talking, to work through awkward emotions and troubling thoughts, precisely because such self-critique might unsettle our own positions. As Madge $(2014,183)$ contends, "it is this vulnerability, this position of unknowing, that might form a bridge upon which a dialogue of mutuality might begin". White's (1992, 165) description of a geopoetic world sensation is that of "a sensation of immensity and incommensurability [...] it means a sense of relativity and topology. It means, globally, a heightened sensitivity towards the environment in which we try to live [and] the acquiring of a non-panic sense of dispersion, disaggregation, dissolution".

Such qualities are as important for the difficult and uncomfortable work of socioecological transformation as they are for decolonisation. The work of decolonisation must identify and validate "multiple ontologies and polycentric epistemologies, is not easy to realise and must be carefully worked towards through everyday practices and relations" (Eshun and Madge 2012, 783). Ahmed (2004) contends that moving towards more pluriversal creative geographies involves adopting a malleable sensibility that is cautious and open. While there is a need to be mindful of the risks of appropriation, there is also a pressing need to compose "socio-natural forms of belonging that enfold and so create worlds otherwise" (Jackson 2014, 76). Perhaps Emergence's 
activities might be seen in such a light as open-ended efforts to compose ancient and new habits of belonging and becoming. As Jackson $(2014,76)$ contends, "in matters concerning being-with others, human and nonhuman, all our everyday materialities hold the capacity to indigenize". In my experience, Emergence's activities of walking and talking cultivate a geopoetical field of presence and activity that is tinged with a sense of care, tenderness, ambiguity and slippage. Perhaps this presents possibilities for ways of dwelling beyond the constricting predicates of an Anthropocene imaginary, and which, therefore, lays the necessary ground work for decolonisation and socioecological transformation.

\subsection{Concluding thoughts on art and socioecologi- cal transformation}

This chapter has explored how two contrasting projects in Wales conceptualise and perform the role of art in socioecological transformation. I have argued that the ways in which we relate to aesthetic/artistic/creative forms are important, not only because these relations reflect and reproduce assumptions about subjectivities in relation to the more-than-human world (e.g. Miles 2014), but also because these practices mobilise people's imaginative capacities in different ways, and thus people's sense of what kinds of Earth futures are desirable and achievable (Castree 2015c). My analysis shows that the Ghost Forest and Emergence represent very different approaches in this respect, the former being concerned with conveying a message about the environment via the spectacle of a particular artwork, while the latter seeks to cultivate "artfulness" as a way of being and doing which itself cultivates personal and ecological awareness and sensibility. In turn, these different approaches might also map on to contrasting Anthropocene imaginaries: the Ghost Forest feeding into a "sublime of environmental destruction", a global imaginary which prompts polar emotions of despair or hope, while Emergence, in many ways, tries to unsettle this homogenising and "flattening" imaginary by making space locally (through walking and talking) for sensing and acknowledging complexity, dissolution, vulnerability, and enchantment. Both projects, however, contain some interesting nuances with regard to how art is mobilised for socioecological transformation: Emergence's activities raise questions about the potential incommensurability of ecological transformation and decolonisation when indigenous ecopoetic creativities are drawn on by "settler" peoples in potentially inappropriate ways. Meanwhile, the Ghost Forest highlights the poten- 
tial malleability of an artwork depending on its context and framing: whereas it was originally intended as a striking message about deforestation and climate change (emphasised by the deathly presence of the trees), in its current location at the NBGW the trees are more a focus for enchantment, play, and touch, and as a metaphorical signpost to the Woods of the World project in an adjacent area of the NBGW. Intriguingly, from the geopoetical perspective on art I outlined in the previous section, it is the planting of the Woods of the World which might be considered more "artful" than the Ghost Forest installation itself, in that such an initiative supports and maintains the emergence of life. Indeed, it resembles a famous project by Joseph Beuys in 1982, called 7000 Oaks, in which Beuys and many volunteers planted oak trees across the city of Kassel in Germany.

Although I have tended to favour a geopoetical approach in this article (as opposed to a Euro-Enlightenment approach to aesthetics), because it helps to close a perceived "gap" between text and world (Jackson 2016), it is worth noting here that, for some, it is precisely this gap that lends art its power to transform. For example, Morton (2007, 25) contrasts Benjamin's theory of art with Adorno's:

For [Adorno], the aesthetic helpfully distances us from something we have a tendency to destroy when we get close to it: The distance of the aesthetic realm from that of practical aims appears inner-aesthetically as the distance of aesthetic objects from the observing subject; just as artworks cannot intervene, the subject cannot intervene in them; distance is the primary condition for any closeness to the content of works. This is implicit in Kant's concept of absence of interest, which demands of aesthetic comportment that it not grasp at the object, not devour it. In this way, the aesthetic promotes nonviolence toward nature. Art is not so much a space of positive qualities (eros), but of negative ones: it stops us from destroying things, if only for a moment. For Benjamin, on the other hand, the aesthetic, in its distancing, alienates us from the world.

I am inclined to agree with Benjamin, because walking and talking with Emergence has helped me to understand art's meaningfulness as a way of doing, being and feeling in everyday life ("artfulness"), as an embodied responsiveness rather than a category of representation and distanced interpretation. In turn, these activities have invited me "to think thoughts that are outside foundational premises of [my] imperial background" (Alfred quoted in Jackson 2016, 19), and to sense the world, and relate to art, in alternative ways. In particular, it has led me to see how artfulness operates 
in the "small stories" (Lorimer 2003), where opportunities to perform and enact the tissues of everyday life differently abound (De Certeau 2011, Ramsden 2017). One important result of re-conceptualising art in such a way is that it redistributes (at least, in theory) transformative agency and capacity amongst people, rather than concentrates it in the hands of a few select artists, or, for that matter, a few powerful politicians. As Guattari ([1989] 2014, 9) suggests, transformation takes place at the micro scale of people's actions and thoughts, and thus there is a need to reinvent our lives "like artists", and Hawkins $(2017,334)$, too, reiterates that "if we are to come close to tackling this global crisis, some creative action is needed".

Unlike Adorno's preference for a distanced aesthetic in the quote above, a geopoetic approach understands the role of art not in terms of representations of, or reflections on, "nature", but rather as part of it (Kroeber 1997, Morton 2007). In this way, art becomes much more than a mechanism for conveying messages or emotions, as its form contributes to shaping socioecological realities just as much as (if not more than) its content. Conceptualising art as "not a thing but a doing" (Barad 2007, 146) is also part of enacting the world-as-becoming rather than fixed in essence, and thus links to the temporal concept of emergence discussed in chapter 3 . Indeed, the idea of artfulness resonates with calls for new paradigms for social change which take seriously "quantum" concepts such as entanglement, uncertainty, complexity and emergence (e.g. O'Brien 2016), and the possibility that, often, "it's the small things that make great change" (O'Brien 2016, 618, quoting the poet, Lemn Sissay). Currently, such small actions tend to be overlooked in current paradigms of transformation, especially in relation to climate change. As O'Brien (2016, 618 619) points out:

much of the current thinking on climate change mitigation is based on the assumptions of classical physics, where agents are discrete individuals or self-interested states that interact through local causation, with little or no role for subjectivity, consciousness, intentionality, and free will. Whether in relation to rational choice theory, game theory, or approaches to global commons and public goods problems, the social world in which climate change responses are deliberated, negotiated, and enacted is ultimately deterministic. If humans are as predictable as matter and material, is there any hope for social change through individual and collective agency?

In a similar way, many mainstream approaches to environmental art might also be understood as adopting a deterministic/cause-effect/top-down approach to transformation. In contrast, the notion of artfulness at least, as I have interpreted it 
resonates with a quantum (physics) perspective on social life which understands humans as "walking wave functions" of potentiality and possibility that intra-act through quantum characteristics, rather than as discrete individuals that interact classically (Wendt 2015, 345). O'Brien suggests that, without taking into account this potentiality and possibility and their non-linear workings, we may be underestimating the capacity of societies to transform. As Barad $(2007,394)$ puts it "[o] ur (intra)actions matter each one reconfigures the world in its becoming". Thus, how we perform our particular wave function matters, because they reverberate in often unexpected or unpredictable ways (this is the analogy that the "butterfly effect" concept makes). I think that this, essentially, is what Emergence tries to accommodate in its notion of "artfulness" a form of agency which is not causally determined but is, as O'Brien (2016) notes "free and full of potential". Although the practicalities of such an approach in terms of changing paradigms for social change in relation to climate change remain to be seen, its metaphorical significance should not be underestimated, because it draws attention to the possibility for individuals to contribute to the making of futures, rather than assume their relative powerlessness in the face of global problems. Specifically, with regard to people's capacities to imagine alternative worlds and futures (e.g. Yusoff and Gabrys 2011, Milkoreit 2016), a "quantum social" approach to art, I suggest, understands imagination as important not so much in the sense that pieces of art can help us to imagine particular futures (as with climate fiction ("CliFi") novels or dystopian movies, for example), but in a rather less instrumental way, as a crucial kind of agency and capacity to think and act in nonlinear and creative ways, individually and collectively. It takes seriously the idea that "everybody is an artist". This is not to dismiss the work of those who professionally identify as "artists", but is rather to argue that, when it comes to socioecological transformation, the imaginative and creative leap required is so great that it cannot be left to those people alone. A quantum approach understands social transformation (of any kind) as involving the creative actions and interactions of many humans (and non-humans). The concept of artfulness is therefore about bringing this agency to people's awareness, and thus available to more conscious cultivation in response to socioecological crises. 


\section{Chapter 6}

\section{Reflections}

During the very first interview anyone gave me for this research project, as I sat in the sun on a makeshift bench amongst pots of herbs outside a run-down static caravan on Gower, sipping lemon and ginger tea and wondering when was appropriate to turn the voice recorder on, the participant turned to me and gave some sage advice. He said that, if I'm interested in environmental crises, then I needed to look at the bigger picture "humanity as a whole, before focussing in on Wales, because the whole ecosystem is connected".

I agreed with him, and still do. Indeed, in the broadest scheme of things, to focus on Wales, and even then on just a handful of projects which involve (at most) a few thousand people, seems like an impossibly minute scale on which to engage with socioecological issues that are global in their production and reach. As Guattari ([1989] 2014, 38) says, "no one is exempt from playing the game of the ecology of the imaginary!" and, indeed, I could have chosen from any number of sites and groups to engage with, which would, no doubt, have resulted in a quite different telling of this story. If my intention had been to conduct a definitive analysis of socioecological transformation, then this partiality would, of course, be quite an oversight. However, as set out in my introduction, I have tried to approach the research from the perspective of "minor theory", which, as Katz points out, "is not about mastery" (Katz 1996, 490) or at least not in the sense of trying to obtain a "complete" picture, or a metanarrative. Instead, it is about being clear about the partiality of my perspective, and of this research project, and it is also about engaging with and taking seriously "the knowledge claims of those working from nondominant positions" (Katz 1996, 488). This is why I have chosen to engage with groups like Emergence and Cae Tan, 
alongside the Welsh Government. It is tempting, as the title of this thesis suggests, to think of these case studies along a spectrum, from the "margins to the mainstream", and it is common to conceptualise these positions as the unimportant as opposed to the important (respectively). But Katz, drawing on Deleuze and Guattari's notion of "becoming-minoritarian", argues that marginality is not that which is "outside", but is the otherness that reworks the mainstream. As she says, "minor theory tears at the confines of major theory; pushing its limits to provoke 'a line of escape', a rupture a tension out of which something else might happen" (Katz 1996, 489). Thus, the marginal is not unimportant, but rather, the marginal and the mainstream, the minor and the major are in constant relation to one another, the boundaries between them always in flux. As Katz suggests, "the two are intertwined in an exquisite and mobile tension. There is no 'last instance' here, but rather a relationship of constant becoming and change" (Katz 1996, 491).

Accordingly, I have started from an understanding of place as made up of heterogeneous relations where and through which possibilities for change in the "margins and the mainstream" might be catalysed (Escobar 2011, Larsen and Johnson 2016). Place-based efforts to bring about socioecological alternatives are indicative of, as Larsen and Johnson $(2016,150)$ say, "partially connected heterogeneous socionatural worlds", but they are also part of a global cosmopolitics, where "cosmos refers to the unknown constituted by ... multiple, divergent worlds and to the articulation of which they would eventually be capable" (Stengers 2005, 995). In other words, the case studies featured in this thesis are necessarily restricted and tightly focused, but they give a glimpse of the coexistence of diverse, often contradictory or radically divergent imaginaries of socioecological transformation. They are a reminder that there is never only "one" story about the future, or about how things could be. My focus on these sites has also been driven by an understanding that the crisis of the Anthropocene, for all its globalness, is also found in the "messy, intimate entanglements of place" (Larsen and Johnson 2016, 162). My aim has, therefore, been to try to understand just a few strands of the kinds of emergent imaginaries and practices that make up these entanglements, rather than to "write down the ultimate equations" (Urry 2005a, 237).

With this in mind, my task in this final chapter is to distil a few of the key themes from each empirical chapter, to draw out some cross-cutting themes and highlight some possibilities for future research directions. I also reflect with the benefit of hindsight on some limitations of the research that might point the way to future research priorities and, last, I wrap up with some concluding thoughts. 


\subsection{Key contributions}

\subsubsection{Time and futurity}

The first of the empirical chapters compared constructions of time and futurity of the Welsh Government, Emergence, and Zero Carbon Britain. In working through this material, I was led to re-assess my own assumption about the necessity of utopianism and future imaginaries an assumption that had sparked the initial idea for this PhD. While the Welsh Government and Zero Carbon Britain both rest on notions of linear time, in which the future is "out there" in front, imagined via goals, pathways, and positive visions of what is possible, Emergence is based on a very different imaginary of time and change. Rather than plotting and planning its way to the future, Emergence extols the value of, well, emergence, which is a particularly "unplanned" approach to time and change. Rather than trying to anticipate a destination, Emergence tries to foster a sense in which newness can arise unexpectedly, although not entirely unconnectedly. Emergence (the concept) refers to how time proceeds "not by continuous growth, smooth unfolding, or accretion, but through division, bifurcation, dissociation by difference through sudden and unexpected change or eruption" (Grosz 1999, 28). As such, Emergence strives to find ways to "live emergently", which involves resisting being constantly preoccupied by the future, and instead trying to slow down in the moment, to be aware of our connection and relationships with other people (through conversations, for example), and to the more-than-human world (through walking in silence, for example). Emergence, and the concept of emergence, also places a degree of trust in the notion of synchronicity, the idea that there are self-organising "tendencies" to how life emerges, which requires that we, as Emergence's co-director, Fern, told me, "step back, silence ourselves and be open to the possibility." Not only does this call into question ways of thinking about the future that require (usually scientific/technological) knowledge about it, but it also seems to promote a sense of more-than-human agency, and an acknowledgement of unknown dimensions of life beyond human control. In addition, this conception of time, change, and agency generates, and requires, a sense of vulnerability which contrasts with the resilience discourse so often depicted in narratives about socioecological transformation (as demonstrated by the Welsh Government). Vulnerability or, as Haraway (2016) puts it, "staying with the trouble", also invites a different way of relating to the world, different sensibilities and subjectivities as Haraway says in one public talk, "we know too little to either despair or hope neither 
is tuned for kinship, complexity, learning". ${ }^{1}$ In contrast, the concept of resilience constantly averts or postpones trouble tacitly in defence of the status quo.

This, however, presents a challenge to the argument that aspirational visions of the future are important for providing orientations in an otherwise disorientating world an argument that has historical as well as recent iterations (e.g. Polak and Boulding 1973, Bloch 1986, Harvey 2000, Nordhaus and Shellenberger 2007, Yusoff and Gabrys 2011). Indeed, it feels somewhat counter-intuitive to disparage the Welsh Government's and ZCB's efforts to construct positive visions of the future (ZCB, in particular, has gone to great lengths to do so), precisely because they do offer a degree of hope and something to aim for. Moreover, the rhetoric of the future is politically powerful, and has become strongly associated with narratives about sustainable development (for example, the UN's sustainable development "goals"). And while it can be argued that this kind of temporality only perpetuates modern ideas of progress and growth, and therefore does little to change imaginaries of time and subjectivity in more fundamental ways, an "emergent" temporality that refuses closure around any particular goal or vision risks perpetuating a "romanticised and perpetually unfilled longing and desire" (Harvey 2000, 183). Perhaps, then, elements of both are helpful. My own view is that socioecological crises leave us with an ethical obligation to think about the future, and I acknowledge that predictions about future environmental scenarios have themselves played a significant role in motivating me to care in the first place. However, perhaps concepts like emergence provide a means through which to transform everyday experiences and ways of perceiving the world that, in fact, slow down or disrupt the kinds of ecologies of ideas (about linear progress, perpetual growth, and of human versus more-than-human agency) that tend to underpin the crises we are currently experiencing. ${ }^{2}$ The concept of emergence can also provide a way of engaging with Levitas's (2013) concept of utopia as method, as an attention to spaces of the lived present as much as a vision of the future, but I think it also points towards the idea of utopia without intention, that is, with a posthuman sensitivity towards formations that arise from the accidental, incidental or contingent (Garforth 2009). In any case, the stark difference between the temporalities of the Welsh Government, ZCB, and Emergence certainly gives pause for thought about what kinds of temporalities sustain what kinds of socioecological transformation.

\footnotetext{
${ }^{1}$ Available at https://www. youtube $. c o m / w a t c h ? v=G r Y A 7 s M Q a B Q$. Last accessed 29 November 2017.

${ }^{2}$ I have written about this in a blog post for Arizona State University's Center for Science and the Imagination. Available at https://medium.com/imaginary-papers/not-thinking-aboutthe-future-of-climate-change-44947840dcb4. Last accessed 29th November 2017.
} 


\subsubsection{Realising relationality}

In chapter 4 , I explored some of the ways in which human/more-than-human relationality is performed in two contrasting examples, the Welsh Government's narratives for socioecological transformation, and Cae Tan's biodynamic approach to agriculture. My analysis indicated that the Welsh Government's claims to relationality although currently in vogue (as in the language of taking an "interconnected" and "joinedup" approach) risk actually reproducing some deeply utilitarian and dualistic ideas about relationships between humans and environment. By contrast, the biodynamic rituals practised by Cae Tan, which although they might appear somewhat "wacky" or superstitious at first glance, actually seem to foster and perform relations with the more-than-human world in a way that recognises interdependence and agency, and fosters a sense of gratitude and even wonder. With that said, Cae Tan tends not to make such practices very visible to the general public or even to most of its members, highlighting, perhaps, a certain stigma that is (still) attached to practices that invoke more-than-human agency. However, these practices enact a complex, dynamic, and "depth-ful" world (in its attention to the layers of soil and its interaction with atmosphere and planetary motions), whereas the Welsh Government's (much more public) imaginary of human environment relations tends to enact a somewhat flattened, two-dimensional, predictable and compartmentalised world that, in actuality, fails to generate any particularly new or more ecological ways of thinking. These findings highlight the importance of critically engaging with narratives of relationality as they arise in the context of the Anthropocene and "after nature" (Mansfield et al. 2015, 284), because "relational" doesn't automatically mean "good", and because these discourses emerge in a number of ways that have various implications for the kinds of social natures they construct, and for the benefit of whom (Mansfield et al. 2015). The examples of Cae Tan and the Welsh Government are also interesting because Cae Tan receives some funding from the Welsh Government. Thus, despite their very different approaches to socioecological transformation, Cae Tan relies on the Welsh Government to some extent, and (presumably) the Welsh Government approves of what Cae Tan does. Perhaps this is a good example of how minor and major theory is always relational, with the constant possibility for minor to push at the limits of major (Katz 1996).

Writing this chapter also highlighted for me the challenge to socioecological transformation presented by language. In particular, finding the language not only to adequately describe but also to perform human environment relationships, that can 
somehow get beyond a binary imaginary of "humans" and "environment". This is vital for moving away from a sense in which people act upon an environment which is "out there", towards a more (genuinely) relational perspective in which human subjectivities are "entangled within and constituted through their more-than-human relationships" (Larsen and Johnson 2016, 153). Perhaps this kind of co-becoming cannot be expressed in words (hence Cae Tan's use of embodied rituals), or is even not accessible by conscious thought at all, thus requiring a "hospitality in thought in the zone of non-knowledge or aknowledge. Such hospitality would challenge what constitutes thinking" (Broglio 2013, 3). Nonetheless, the contrasting ways in which the Welsh Government and Cae Tan talk about, and practice, human and more-thanhuman relationality highlights the potential heterogeneity of imaginaries that exist, and the centrality but also the limits of language in how these imaginaries are performed and conveyed. Taken together, chapters 3 and 4 present novel analyses of the Welsh Government's narrative of socioecological transformation, juxtaposed with examples from more "marginal" case studies that are imagining transformation otherwise.

\subsubsection{The role of art in socioecological transformation}

In the final empirical chapter I homed in on the topic of art, and its role in bringing about socioecological transformation through the ways in which art mediates relations between humans and the more-than-human world. This interest was brought about through my engagement with Emergence and with the Ghost Forest environmental art installation at the National Botanic Garden of Wales; projects that approach art in relation to socioecological crises and transformation in quite different ways. The chapter contributes to current discussions (in geography and beyond) about art and environment, by questioning what art "is" and "does" and who does it in relation to socioecological crises, and how, by conceptualising these things differently, there might be implications for the kinds of transformations art facilitates. In particular, I suggest that the Ghost Forest art installation represents a widespread tendency to use and engage with art as a spectacle, or as a vessel through which to communicate a particular environmental message. It relies on ideas about a distinct artwork, created by an innovative artist, which is viewed by an audience. This is a classically Western-Enlightenment approach to aesthetics, where art is conceptualised as something which requires (or assumes) a degree of distance and judgement on the part of the viewer, and genius and creativity on the part of the artist. This dualistic 
approach to artist/viewer, subject/object can be criticised for the ways in which it perpetuates broader dualisms such as nature/culture, and subject/object.

In contrast, I suggest that Emergence's approach to art might offer some alternative modes of socioecological transformation, by conceptualising art not as a "thing" but a "doing". Emergence draws on the idea of artfulness to develop a sense in which everyone is an "artist", and artfulness is a way of engaging with the world which fosters a sense of interdependence, creativity, improvisation, and the ability to be receptive to emergent phenomena (to link back to themes on temporality, above). In Emergence's approach, this is not an art which is reserved for galleries but is something which everyone participates in, whether they are cooking, talking, or cleaning (to name but a few activities). My experience with Emergence indicates that, in attempting to dismantle fixed ideas about what art is and who does it, it is possible to generate different kinds of sensibilities and subjectivities in and of the world and in particular, a sense of dwelling as well as a sense of empowerment in terms of how individuals perceive their own ability to transform. It also challenges a focus on the global as the site of socioecological transformation, and emphasises the everyday and the local as important sites of transformation and engagement, too. The chapter emphasises that there may be a link between how we think about art, and how we think about the environment (e.g. Miles 2014), a sentiment summed up by Naess's comment that, "to 'only look at' nature is extremely peculiar behaviour. Experiencing an environment happens by doing something in it, by living in it, meditating and acting" (Naess 1989, 63).

However, Emergence's approach is not without some contentions. In particular, this chapter builds on literature on geopoetics (a concept which broadly aligns with Emergence's approach) by discussing some potential issues of cultural appropriation. Given that Emergence's approach to art diverges significantly from a WesternEnlightenment model of aesthetics, it is perhaps not surprising that it draws on a variety of Welsh and other (particularly Native American) indigenous practices, and this leaves open questions as to how alternative modes of art and socioecological relations might be practised in Western contexts without reproducing colonial forms of appropriation and harm. In addition, I suggest that, while Emergence's activities might contribute to decolonial thinking in that they disrupt dualistic and utilitarian imaginaries of the world, these practices might in fact be incommensurable with $a c$ tual decolonisation because they do not address the problem of continued abuse of land rights in various parts of the world (this is not considered as much of problem in Wales, where language is the central issue, but is very much central to decolonial 
struggles elsewhere) (Tuck and Yang 2012). This is sensitive territory which I am still working through and trying to understand. As a white, British person who is also a "settler" in Wales, my engagement with Emergence and writing this chapter (and a paper associated with it), has forced me to consider my own position in a very different light, and often in uncomfortable ways. It is perhaps for this very reason the discomfort of it that the intersection of art, socioecological transformation, indigenous ontologies and decoloniality is a topic that is worthy of much further thought and engagement, as I think that there is much to be learned.

\subsection{Overarching themes}

\subsection{1 "Social physics"}

Interestingly, many of the case studies I have engaged with share a certain tendency towards what Urry $(2003,236)$ calls "social physics", that is, social iterations of "new science" approaches such as quantum physics and complexity theory. These approaches challenge a classical physics understanding of the world based on linear notions of cause and effect, and instead emphasise the centrality of entanglement, uncertainty, non-linearity, energy and relationality in how change over time occurs (Barad 2007). In essence, the complexity turn (Urry 2005b) sees a shift from thinking of phenomena as separate and separable, to thinking of them as connected and inseparable (Cambray 2002).

For example, all of the case studies express an interest in notions of "interconnectedness", even if, in some instances, this is only superficially incorporated into their actions and narratives. For example, the Welsh Government is adept at incorporating a language of interconnectedness and joined-up thinking into its policy frameworks (the Well-being of Future Generations Act (2015) merges the social, environmental, and economic legs of the traditional sustainability "stool") but, as previously noted, one can detect a persistence of Enlightenment thinking in its linear conceptions of time and goal-orientated futures, as well as in its focus on human-only agency. In contrast, Cae Tan's performances of human/more-than-human relations via its biodynamic practices enact a complex and dynamic world full of more-than-human agency, and an intricate understanding (which is not necessarily only intellectual, but intuitive, too) of the interplay of organic, non-organic and cosmic energies. Meanwhile, Emergence adopts a "complexity" understanding of the world through its rejection of 
notions of linear time and determinism, instead preferring to understand transformation as brought about in often unpredictable, sometimes imperceptible, perturbations and iterations which "emerge" rather than follow a known or planned course. Emergence also seeks to foster connectivity between people through conversations, and between people and the more-than-human world through walking outside.

In addition, Emergence and Cae Tan demonstrate a sensitivity towards understanding the world in terms of flows of energy, which also resonates with "new science" understandings. For example, Cae Tan pays attention to the transmission of atmospheric energies (high and low pressure) to the soil, and how this influences growth and activity through the seasons. Emergence, too, incorporates notions of synchronicity into its work, and synchronicity as Jung first proposed it is basically understood as "meaningful coincidence" as a phenomenon of energy (Cambray 2002). Similarly, Emergence's focus on face-to-face conversations is one that recognises the subtle exchanges of energy that occur through the voice and embodied encounters. In a different way, Zero Carbon Britain's work is also highly attuned to energy, not only with respect to renewable energy, and re-connecting with these sources of power, but also in a faintly spiritual sense, in that it centres its story of human development on the subject of the sun.

An acceptance of uncertainty also aligns with some "new science" understandings of the world. My analysis of Emergence and Cae Tan indicates that elements of uncertainty and mystery are what sustains their practices. The very concept of emergence accepts that the future cannot be known, and posits uncertainty as the wellspring of genuine newness, and genuine potential for transformation (Grosz 1999). Indeed, this is why Emergence frequently seek to facilitate conversations between strangers, as this creates conditions for surprising and unexpected interactions. Cae Tan's biodynamic rituals also retain a sense of mystery, both in the sense of an element of spirituality that is present in its work, and in relation to whether and how the biodynamic preparations make a difference. For several of the volunteers, the mystery of the biodynamic process was, in fact, part of the attraction. They didn't need to know how or even if the preparations "worked", and this uncertainty created a sense of wonder. In contrast, the Welsh Government and Swansea Council have constructed frameworks which privilege knowledge about the future (for example, through the Future Trends reporting scheme). This is a tactic which can be linked to the resilience discourse these organisations also employ, but which in fact could be seen as a misinterpretation, or co-option, of the idea of resilience which in ecological sciences, at least is intended to correspond with a complex, emergent view of life (e.g. Folke et al. 2010). 
In the Welsh Government's approach, uncertainty is approached predominantly via a risk assessment-style approach, and as something to be minimised or contained.

Finally, Emergence's concept of artfulness as "not a thing but a doing" (Barad 2007, 146) might also be thought of as corresponding with a quantum/complex philosophy of matter and transformation. ${ }^{3}$ For example, the notion of everyday artfulness in the small, even mundane, aspects of life (such as walking and talking), is a way, perhaps, of understanding humans (and non-humans) as "walking wave functions" of potentiality and possibility that interact through quantum characteristics, rather than as discrete individuals that interact classically (Wendt 2015, 345). Artfulness implies an ability to live, as Guattari ([1989] 2014, 9) says, like an artist that is, with qualities such as improvisation, creativity, intuition and flexibility. It is also to acknowledge the interplay of human agency with more-than-human agency, and the possibilities and potentialities that arise from this. Thus, artfulness is a means by which to begin to live a different kind of temporality to a linear one, with a sensitivity and openness to emergence, becoming, and uncertainty. Alternatively, artfulness might be thought of in relation to Haraway's "tentacular thinking" a material and cognitive practice of learning, sensing, becoming-with, and which represents a more messy, asymmetrical, and rhizomatic imaginary of time and space than an impoverished one that is restricted to linear or conical geometries (Haraway 2016). Indeed, the notion of everyday artfulness is a thread which links many of the themes in this thesis. Although in chapter 5 I have begun to draw out some of the implications of this concept in relation to the complexity turn, and to research on and in geopoetics, this certainly feels to me to be a line of enquiry worthy of further exploration. In particular, the question of whether, as Madge $(2014,180)$ asks, poetry (in the broadest sense) is "a creative process of thoughtful making, an act of expressing new and imaginative ideas and feelings that can be undertaken by anyone, or is it an aesthetic practice that can only be performed by those with particular skills or formal training?" is central to questions about socioecological transformation, how it happens, under what circumstances, and undertaken by whom. As O'Brien $(2016,619)$ contends, "quantum social theory" challenges current paradigms of social change that are based on assumptions of classical physics (where agents are discrete individuals or states that interact through linear pathways of cause effect), by presenting the possibility that relatively small phenomena combine to produce much larger and hitherto unexpected effects. Of course, this could be equally true for the potentially catastrophic (and unknown) effects of climate change as it is for any more

\footnotetext{
${ }^{3}$ Whereas, the Western-Enlightenment model of aesthetics aligns more closely with a classical physics perspective in terms of binary approaches to subject/object, cause/effect)
} 
positive socioecological transformations. But it does mean that, possibly, the opportunities for transforming to alternative socioecological arrangements are greater than we think, or have been led to believe are possible or feasible (O'Brien 2016). The metaphorical impact of such a statement, and of the associated notion of everyday artfulness, is as potentially significant as its practicalities, because it offers a mode of thinking that might empower us in a situation that otherwise seems to render us helpless (Stenstad 2009). The concept of everyday artfulness could contribute to the creation of subjectivities capable of dissensus ("a revival of individual competence as a social force" (Guattari [1989] 2014, 11), that are simultaneously enmeshed in wider collective responsibilities. This is a subjectivity that is, as Fisher $(2009,66)$ argues, one that is permanently deferred from emerging by the impersonal and consensual structures of contemporary life, but it is one that is urgently needed.

\subsubsection{The good, the bad, or the post-Anthropocene?}

The case studies explored in this thesis can also be thought of as indications as to how narratives, imaginaries and ethical relations for and of the Anthropocene are taking shape. Interestingly, despite growing use of the term in scientific literature about environmental change, in my experience none of the case studies nor the people I spoke to referred to the Anthropocene by name. While this could be taken as an indication that the term is not entering common parlance and becoming a societal "key word" as fast as Castree (2014a) and others have anticipated, it is also possible that the Anthropocene, that is, as signifying a radical shift in "species awareness" (e.g. Chakrabarty 2009), is, nonetheless, shaping approaches to socioecological transformation.

The Welsh Government comes closest to evoking the Anthropocene directly, in the sense that it has aligned itself with discourses about planetary boundaries, environmental limits, and ecological footprints (e.g. Rockström et al. 2013) that are currently at the heart of geoscience-dominated iterations of the Anthropocene (see Castree 2014a). In addition, the Welsh Government's brightly-coloured, optimistic depictions of desirable futures in its public communications and short films also seem to adopt an ecomodernist-style stance towards the makings of a "good Anthropocene", based largely on techno-managerial adaptations, and the centrality of scientific evidence as a basis from which to act (see Nordhaus and Shellenberger 2007, Ellis 2011). The "good Anthropocene" hypothesis is largely characterised by an acceptance of 
environmental-change science, but rather than framing it as a disaster, it frames it as an opportunity for triggering new forms of prosperity and resilience (Dalby 2015). Zero Carbon Britain also adopts this kind of techno-optimism, a sense in which there is still a chance just to keep global warming below two degrees Celsius, although its own solutions, arguably, are much more rigorously thought out and commensurate with what climate science does actually deem necessary, than are the Welsh Government's aspirations.

In contrast, Emergence's practices seem to be rooted in an acknowledgement that current socioecological circumstances are already bad, and likely to get worse. This doesn't mean that it doesn't seek to try to improve the situation, but, equally, it doesn't proclaim that "saving" the situation, or averting a catastrophe, is a possibility. Instead, much of its narrative places us firmly in the midst of catastrophe, and its work is about trying to find ways of coping with and responding to this, particularly through acknowledging our own vulnerability to what is happening. Its concern seems to be less with how to continue present ways of life, but rather with "the deeper challenge of crafting new ways to respond with honour and dignity to unruly earth forces" (Ginn 2015, 7). In doing so, Emergence challenges a current vogue for a "keep calm and carry on" mentality that characterises the ecomodernist visions of a good Anthropocene (Latour 2015).

In another sense, Emergence and Cae Tan, in particular, might be thought of as "postAnthropocene" responses to socioecological crises (e.g. Bratton 2013, Colebrook 2014, Wallin 2017), because they both generate the kinds of imaginaries which could be read as rebuttals of the largely anthropocentric and science-centric diagnoses of the current epoch. Both, for example, are orientated towards a recognition of morethan-human agency, and of human dependence on, and gratitude for, these morethan-human agencies. This is in contrast with the Welsh Government, which depicts human relationships with the more-than-human world almost entirely in terms of resources, ownership, and management, thus lending support to the criticism that the Anthropocene merely reproduces very anthropocentric discourses that focuses on the rights and well-being of human life over all others (e.g. Chernilo 2017). In addition, Cae Tan and Emergence both reject science as the sole form of knowledge (although they are not anti-science), as they both incorporate other forms of knowledge gnosis, or intuition, for example into their practices. ${ }^{4}$ For Emergence, this is apparent

\footnotetext{
${ }^{4}$ It is interesting to note that both Cae Tan and Emergence share a heritage of ideas from Rudolf Steiner: Steiner was the founding figure of biodynamics, and he also greatly influenced the German artist, Joseph Beuys, whose argument that "everybody is an artist" has been an important inspiration for Emergence's approach. Central to Steiner's thinking was the importance he placed
} 
through its references to and incorporation of the idea of synchronicity, and for Cae Tan it can be seen in its biodynamic rituals. Such rituals might be considered by many as a pseudo-science, but they play an important performative, social, and psychological role in how the more-than-human world is perceived and related to. In short, Cae Tan and Emergence seem to represent attempts at post-human thought, thus resisting some of the Euro-modernity framings of the Anthropocene centred on the privileging of scientific knowledge and human agency and rights. Rather than rely on "ontological monism" (Larsen and Johnson 2016, 162) to set the terms of debate in this epoch, Castree $(2015 \mathrm{c}, 6)$ suggests that, "a much broader framing is called for ... that explores how 'problems' of, and 'solutions' to, global environmental change look when we examine them through complex virtues like wisdom, humility, integrity, faith, hope, and love". In this sense, Cae Tan and Emergence offer glimpses of more pluriversal ways of thinking about, and responding to, socioecological crises, and what kinds of futures are possible beyond the register of the Anthropocene. Zero Carbon Britain, too, toys with notions of spirituality, but there is a sense that like Cae Tan it is hesitant to be too public about this, for fear of undermining the project's technological credibility. This raises an interesting question in itself, namely, how might these different forms of knowledge science, technology, and spirituality find ways to co-exist, ontologically, and what kinds of socioecological possibilities might this give rise to? In this light, perhaps Emergence and Cae Tan's approaches might be read as "poetic political" (Springer 2017, 4) projects which attempt to combine various knowledges, with feeling, and even with non-knowledge (Bataille 2004), thus helping to break with the colonial knowledges which restrict our diagnostics of the present moment, and therefore of the potential futures we imagine (Blaser 2009).

\subsection{Limitations}

As I hope I have established by now, my approach to this research, and to the writing of it, has been very much grounded in an acknowledgement of my own partiality, and the limitations but also the benefits that come with such an approach. However, with the benefit of hindsight there are two issues, in particular, which I feel have shaped how the thesis has turned out, and which I might have approached differently if I were to start again.

on spirituality, imagination, intuition and sensory experience (his philosophy of anthroposophy attempted to bring this together with Western science). 
The first of these relates to citational politics. There are always questions of politics and power wrapped up in the doing and writing of research (Marston and De Leeuw 2013). Geographical work enacts, performs, and presents the world as much as it represents or explains. With regard to the case studies I have chosen to engage with and write about, I have taken this proposal seriously throughout. However, it has taken me somewhat longer to realise that the works I cite are just as much a part of this world-building as the subjects of the research itself. This was brought to my attention, in particular, through writing chapter 5 , on the role of art, which engages with debates about decolonialism. Just as if I had chosen a different selection of case studies, a different selection of citations and works through which to construct my ideas would also, undoubtedly, have resulted in a different thesis. I have not counted, but I hazard a guess that the thesis is overwhelmingly dominated by citations of work by white, Western, predominantly male, academics. Critical reflection is needed about this, when one considers that citation is, as Ahmed writes "a rather successful reproductive technology, a way of reproducing the world around certain bodies" 5 (see also Mott and Cockayne 2017, Radcliffe 2017a). Relatedly, a regrettable omission from this thesis, given its geographical location, has been Welsh language, both in the ethnographic and interview material I have generated, and in my citations. This is because I do not speak Welsh, and I am increasingly aware of how my reliance on English as my only means of communicating and reading research is a significant limitation in my being able to participate in pluriversal understanding and scholarship (Radcliffe 2017a). Had I conducted the research in Welsh, I might have gained a very different perspective on things. For example, one interviewee (whom I interviewed in English, although he was himself a Welsh speaker, too), I later heard giving an informal interview (again, in English) on BBC Radio 4, in which he warned the interviewer that if he were to start talking in Welsh it might set him off crying because, for him, "Welsh comes from the heart". 6 This comment indicates the importance of language in enacting different worlds and emotions, and the restrictions that come with only being able to experience these through one language.

The second potential oversight that I would like to briefly draw attention to has been my tendency to approach the research from a perspective that favours affirmative narratives and ontologies of becoming, in line with a "vitalist" Spinozist and DeleuzoGuattarian ethos. ${ }^{7}$ Although this is part of the kind of ethics which I wanted, as a

\footnotetext{
${ }^{5}$ Available at https://feministkilljoys.com/2013/09/11/making-feminist-points/. Last accessed 2 December 2017.

${ }^{6}$ Available at http://www.bbc.co.uk/programmes/b07z3hpt. Last accessed 2 December 2017.

${ }^{7} \mathrm{I}$ am drawing here on the framing of a recent call for papers for a session entitled "Besides affirmation: geography and "negativity"' at the Association of American Geographers An-
} 
researcher, to participate in (as outlined in chapters 1 and 2), it is worth noting that this approach can be criticised for potentially reproducing exactly the kinds of neoliberal expectations and subjectivities (centred on productivity) that the affirmative approach often claims to resist (e.g. Gibson-Graham 2008). An alternative approach might have engaged, for example, with negation and negativity (e.g. Thacker 2015) as a means to think through socioecological imaginaries that question what is lost when expectations of connectivity, creativity, joy and hope are normalised (e.g. Osborne 2003, Ahmed 2010, Culp 2016), and this, too, might have brought to light, and performed, some very different stories of socioecological transformation. Relatedly, it is important to note that this research has largely engaged with the socioecological projects and imaginaries of relatively affluent people living relatively comfortable lives. While I do not wish to suggest that this somehow reduces their credibility, it does raise questions about the geographical disparity in who is imagining and driving socioecological transformation who has the energy, the resources, the networks, and who is left out of the picture (Braun 2015a)? For example, Wales has some of the highest levels of poverty in the UK (Joseph Rowntree Foundation 2015, 2016), and yet these crises of poverty feature very little in the stories I have engaged with here a consequence of my own choices of focus as much as the case studies themselves.

\subsection{Concluding thoughts}

In a timely publication, Ripple et al. (2017) have issued "World scientists' warning to humanity: A second notice". The journal article has 150,364 scientist signatories, more than has been recorded for any other journal article. Accordingly, it has already received huge global media attention, under such catchy headlines as "Thousands of scientists issue bleak 'second notice' to humanity". 8 And so the pitch of the socioecological crisis alarm bell rises another semitone. Sadly, however, the article contains very little input from humanities or critical social science, and the "intellectual climate" (Castree 2015b, 244) in which the issues are discussed seem to have moved on little from discussion in the "first warning", published some 25 years ago. Indeed, in

nual Meeting 2018. See https://distortedspace.com/2017/09/21/call-for-papers-besidesaffirmation-geography-and-negativity/. Last accessed 2 December 2017.

${ }^{8}$ Available at https://www.washingtonpost.com/news/speaking-of-science/wp/2017/11/ 13/thousands-of-scientists-issue-bleak-second-notice-to-humanity. Last accessed 3 December 2017. 
a much less-publicised blog-post, ${ }^{9}$ De Rosa and Bluwstein (2017) offer a stiff rebuttal of the article, claiming that it "gets the socioecological crisis (and its solutions) wrong". Central to their claim, which is based in political ecology, is that Ripple et al. propose elitist "solutions" (population reduction; technology; protected areas; diet; and more) that fail to acknowledge the root causes of socioecological crises, and, worse, perpetuate some imperial and racist discourses. Moreover, they suggest, the article addresses a singular "humanity", from the position of a collective scientific authority a dangerous illusion that erases "the deeply uneven socio-ecological and metabolic patterns that underpin the lives of people who are living at different heights of the per-capita consumption and emission ladder", and which also places themselves the scientists as the sole gatekeepers' of knowledge about the earth. Against this, De Rosa and Bluwstein ask why, for example, do Ripple et al. not mention neoliberal capitalism as a root cause? Why create an "overpopulation bogeyman" (aimed at the Global South) rather than call on the most privileged of the world's population (those with the greatest per capita consumption) to accept responsibility and to join in with the multitude of grassroots struggles already underway?

This exchange highlights how the framing of socioecological crises is itself a field of struggle. It is this field of struggle that I have, in a small way and with a few specific examples, tried to engage with, and participate in, in this thesis. I have shown how framings of time, futurity, human environment relations, and art and aesthetics, underpin various approaches to socioecological transformation, and how, in turn, these can be traced to various or are perhaps part of forming new ecologies of ideas. In particular, I have focussed on how these ideas are performed and go to work, and thus what kinds of transformations and socioecological futures they make possible. Performance, as Kershaw contends, is a form of energy in the world no organisms exist without performing, and so "the precise nature of their performance is a matter of no small consequence" (Kershaw 2012, 276). Through this research, I have presented a novel analysis of the Welsh Government's own particular performance of socioecological transformation, and the ecologies of ideas that sustain it. Geopolitics is part of how the world is made known (Last 2017), and the Welsh Government offers some important indications as to how wider imaginaries for the "Anthropocene" and socioecological transformation are unfolding. I admire the Government's ambition and innovation in introducing an Act that is explicitly aimed at changing how the environment is related to in law, something that few governments have ventured to do. But I question whether the language and ideas it uses to bolster the Act are capable of

\footnotetext{
${ }^{9}$ Available at https://entitleblog.org/2017/11/23/why-warning-to-humanity-gets-thesocio-ecological-crisis-and-its-solutions-wrong. Last accessed 3 December 2017.
} 
bringing about anything more than some incremental changes in management style, rather than more radical shifts in socioecological arrangements particularly when it comes to recognising more-than-human agency, and fostering human environment relationships based on something other than economic and utilitarian premises.

But perhaps the Welsh Government, despite its utopian vision (albeit somewhat complicated by narratives of resilience and environmental limits) is not where one should expect, or hope, to find truly radical ruptures with the status quo in any case. This thesis has considered other sites as potential examples of "heterotopias" (Foucault [1966] 1970), that is, as processes of otherness in the present that experiment with alternative ecologies of ideas, presenting "lines of escape" (Katz 1996, 489) from the norms that otherwise tend to imprison imagination. The relationship of these case studies to the Welsh Government is not a straightforward case of "good versus bad" (at least, I have tried to resist presenting it as such), but rather a matter of how the minor and the major the margins and the mainstream are entangled, and what kinds of cosmopolitics and social-natures these entanglements might produce (Stengers 2005). However, the question of where to go from here, in practical terms, remains. How might some ideas about socioecological transformation "from the margins" gain more traction, or even be incorporated into mainstream politics? What would be the effect of doing so (or does the promise of minor theory depend on it remaining "marginal"?). From the perspective of ecologies of ideas (Guattari [1989] 2014), perhaps the single most important thing to do is to share the ecologically "good" ideas so that they might begin to crowd out the bad ones. This means more conversations between people from different "silos" of society artists and policymakers, for example. This is already happening to a small extent but could happen much more. Guattari's position was that changes at the level of people's own mental ecologies is as important for socioecological transformation as institutional-level change, and that mental ecologies can only change through encounters with new and different ecologies of ideas. Placing trust in the affective lives of ideas is an admittedly less comfortable stance to adopt, for it is never clear how and if such ideas will take hold. As Solnit $(2005,66)$ reminds me, action is seldom direct seeds can be scattered, but "rats might eat them, or they might just rot, [and] some seeds lie dormant for decades because they only germinate after fire". Such uncertainty requires an ability to hesitate, to slow down, to be "OK" with not knowing although this is precariously balanced with a concurrent need to resist apathy, and to actively protect and foster the kinds of ideas that are desirable for socioecological transformation when and if we come across them. 
CHAPTER 6. 


\section{Bibliography}

Abram, D., 1997. The Spell of the Sensuous: Perception and Language in a MoreThan-Human World. Vintage.

Abram, D., 2014. The Commonwealth of Breath. In S. Iovino and S. Oppermann, eds., Material Ecocriticism, 301 314. Indiana University Press.

Adam, B., 2013. Time and Social Theory. John Wiley \& Sons.

Adam, B. and C. Groves, 2007. Future Matters: Action, Knowledge, Ethics. Leiden and Boston: Brill.

Adam, B. and C. Groves, 2011. Futures tended: Care and future-oriented responsibility. Bulletin of Science, Technology \& Society 31 (1): 1727.

Adams, C. J., 2015. The sexual politics of meat: A feminist-vegetarian critical theory. Bloomsbury Publishing USA.

Adey, P., 2015. Air's affinities: geopolitics, chemical affect and the force of the elemental. Dialogues in Human Geography 5 (1): 5475.

Adorno, T. W., 1997. Aesthetic Theory. A\&C Black.

Agamben, G., 1993. Time and History: Critique of the Instant and the Continuum. Verso.

Agamben, G., 2004. The Open: Man and animal. Stanford university press.

Ahmed, S., 2004. Cultural Politics of Emotion. Edinburgh University Press.

Ahmed, S., 2010. The Promise of Happiness. Duke University Press.

Allen, P., L. Blake, P. Harper, A. Hooker-Stroud, P. James and T. Kellner, 2013. Zero Carbon Britain: Rethinking The Future. Centre for Alternative Technology.

Allen, P., E. Hinshelwood, F. Smith, R. Thomas and S. Woods, 2014. Culture Shift: How Artists are responding to Sustainability in Wales. Emergence.

Allouche, J., C. Middleton and D. Gyawali, 2015. Technical veil, hidden politics: Interrogating the power linkages behind the nexus. Water Alternatives 8 (1): 610 626. 
Alm, S., 2011. The worried, the competitive and the indifferent Approaches to the future in youth, their structural roots and outcomes in adult life. Futures 43 (5): 552562 .

Altena, A., M. Belina and L. van der Velden, 2015. The Geologic Imagination. Sonic Acts, Amsterdam, NL.

Altvater, E., 1993. The Future of the Market: An Essay on the Regulation of Money and Nature After the Collapse of" actually Existing Socialism". Verso.

Amin, A. and N. Thrift, 2005. What's left? Just the future. Antipode 37 (2): 220238.

Anderson, B., 2006a. Imagined communities: Reflections on the origin and spread of nationalism. Verso Books.

Anderson, B., 2006b. "Transcending without transcendence": utopianism and an ethos of hope. Antipode 38 (4): 691710.

Anderson, B., 2009. Affective atmospheres. Emotion, Space and Society 2 (2): 7781.

Anderson, B., 2010. Preemption, precaution, preparedness: Anticipatory action and future geographies. Progress in Human Geography 34 (6): 777798.

Anderson, B. and P. Adey, 2012. Future Geographies: Guest Editorial. Environment and Planning-Part A 44 (7): 15291535.

Anderson, B. and J. Fenton, 2008. Editorial introduction: Spaces of hope. Space and Culture 11 (2): 7680 .

Anderson, B. and P. Harrison, 2010. The promise of non-representational theories. In B. Anderson and P. Harrison, eds., Taking-place: non-representational theories and geography, 1 36. Ashgate.

Anderson, B., M. Kearnes, C. McFarlane and D. Swanton, 2012. Materialism and the politics of assemblage. Dialogues in Human Geography 2 (2): 212215.

Anderson, B. and C. McFarlane, 2011. Assemblage and geography. Area 43 (2): 124127.

Anderson, K., 2013. Hypocrites in the air: Should climate change academics lead by example. http://kevinanderson.info/blog/wp-content/uploads/2013/ 04/Hypocrites-in-the-air-should-climate-change-academcis-lead-byexample2.pdf. Last accessed 2 August 2017.

Anderson, K., 2014a. Is flying still beyond the pale? https://newint.org/ sections/argument/2014/01/01/flying-still-beyond-the-pale/. Last accessed 2 August 2017.

Anderson, K., 2014b. Mind over matter? On decentring the human in Human Geography. Cultural Geographies 21 (1): 318. 
Anderson, K. and A. Bows, 2008. Reframing the climate change challenge in light of post-2000 emission trends. Philosophical Transactions of the Royal Society of London A: Mathematical, Physical and Engineering Sciences 366 (1882): 3863 3882 .

Andreas, M. and F. Wagner, 2012. Realizing Utopia. Ecovillage Endeavours and Academic Approaches. RCC Perspectives .

Appadurai, A., 1996. Modernity at Large: cultural dimensions of globalization. Minneapolis, USA: University of Minnesota Press.

Appadurai, A., 2013. The future as cultural fact. Verso.

Aradau, C. and R. Van Munster, 2011. Politics of Catastrophe: Genealogies of the Unknown. Routledge.

Armbruster, K., 2011. Into the wild: An ecofeminist perspective on the human control of canine sexuality and reproduction. In D. A. Vakoch, ed., Ecofeminism and rhetoric: Critical perspectives on sex, technology, and discourse, 39 64. Berghahn Books.

Arnaldi, S., 2008. School, images of the futures and social processes in classrooms. Futures 40 (9): 795802.

Atkinson, P. and A. Coffey, 1997. Analysing Documentary Realities. In D. Silverman, ed., Qualitative Research: Theory, method and practice, 45 62. London: Sage.

Austin, J. L., 1975. How to do things with words. Oxford University Press.

Bakker, K., 2010. The limits of "neoliberal natures": Debating green neoliberalism. Progress in Human Geography 34 (6): 715735.

Ballengee-Morris, C., 2008. Indigenous aesthetics: Universal circles related and connected to everything called life. Art Education 61 (2): 3033.

Bannon, B. E., 2011. Re-Envisioning Nature: The Role of Aesthetics in Environmental Ethics. Environmental Ethics 33 (4): 415436.

Barad, K., 2003. Posthumanist performativity: Toward an understanding of how matter comes to matter. Signs: Journal of women in culture and society 28 (3): 801831.

Barad, K., 2007. Meeting the universe halfway: Quantum physics and the entanglement of matter and meaning. Duke University Press.

Barnes, T. J., 2008. American pragmatism: Towards a geographical introduction. Geoforum 39 (4): 15421554.

Barnett, C., 2015. On the milieu of security: situating the emergence of new spaces of public action. Dialogues in Human Geography 5 (3): 257270. 
Barthes, R., 1972. Mythologies, trans. by A. Lavers. New York: Hill and Wang.

Bartolini, N., R. Chris, S. MacKian and S. Pile, 2016. The place of spirit: Modernity and the geographies of spirituality. Progress in Human Geography 41 (3): 338354.

Bartram, R., 2005. Nature, art and indifference. Cultural Geographies 12 (1): 117.

Bataille, G., 1988. The Accursed Share. An Essay on General Economy, trans. by R. Hurley. New York: Zone Books.

Bataille, G., 2004. The Unfinished System of Nonknowledge, trans by S. Kendall and M. Kendall. University of Minnesota Press.

Bateson, G., 1972. Steps to an Ecology of Mind: Collected essays in anthropology, psychiatry, evolution, and epistemology. University of Chicago Press.

Bateson, G., 1979. Mind and Nature: A Necessary Unity. Dutton New York.

Baudrillard, J., 2000. The Vital Illusion. Columbia University Press.

Bauman, Z., 1993. Modernity. In J. Krieger, ed., The Oxford Companion to Politics of the World, 592596.

Bauman, Z., 1996. From Pilgrim to Tourist or a Short History of Identity. In S. Hall and P. du Gay, eds., Questions of Cultural Identity, 19 38. London: Sage.

Bauman, Z., 1997. Postmodernity and its Discontents. Polity Press.

Bauman, Z., 1999. In Search of Politics. California, USA: Stanford University Press.

Bauman, Z., 2000. Liquid Modernity. Cambridge, UK: Polity Press.

Bauman, Z., 2003. Utopia with no topos. History of the Human Sciences 16 (1): 1125.

Bauman, Z., 2007. Liquid Times: Living in an Age of Uncertainty. Polity, Cambridge.

Beck, J. and M. Dorrian, 2014. Postcatastrophic utopias. Cultural Politics 10 (2): 132150 .

Beck, U., 1992. From industrial society to the risk society: questions of survival, social structure and ecological enlightenment. Theory, culture \& society 9 (1): 97123.

Beck, U., 1995. Ecological Politics in an Age of Risk. Germany: Polity Press.

Beck, U., 1999. World Risk Society. Cambridge, UK: Polity.

Beck, U., 2006. Cosmopolitan Vision. Polity.

Beck, U., 2010. Climate for change, or how to create a green modernity? Theory, Culture 85 Society 27 (2-3): 254266. 
Beck, U., 2016. The metamorphosis of the world: How climate change is transforming our concept of the world. John Wiley \& Sons.

Belfiore, E., 2013. Response 1. In The Art of Life: Understanding how participation in arts and culture can affect our values. Mission Models Money and Common Cause.

Benjamin, W., [1935] 2008. The work of art in the age of mechanical reproduction. Penguin UK.

Benjamin, W., [1942] 2009. On the Concept of History. Classic Books America New York.

Bennett, J., 2001. The enchantment of modern life: attachments, crossings, and ethics. Princeton University Press.

Bennett, J., 2007. Edible Matter. New Left Review 45: 133145.

Bennett, J., 2009. Vibrant matter: A political ecology of things. Duke University Press.

Bergson, H., 1998. Creative Evolution. Dover Publications.

Berkes, F., J. Colding and C. Folke, 2008. Navigating social-ecological systems: building resilience for complexity and change. Cambridge University Press.

Berkes, F. and C. Folke, eds., 1998. Linking social and ecological systems for resilience and sustainability. Cambridge University Press.

Berlin, I., 2003. The Crooked Timber of Humanity: Chapters in the History of Ideas. London: Pimlico.

Bernstein, R. J., 1992. The resurgence of pragmatism. Social Research 813840.

Bingham, N., 1996. Object-ions: from technological determinism towards geographies of relations. Environment and Planning D: Society and Space 14 (6): 635657.

Bingham, N. and S. Hinchliffe, 2008. Reconstituting natures: Articulating other modes of living together. Geoforum 39: 8387.

Bird Rose, D., 2013. Slowly Writing into the Anthropocene. TEXT 20: 114.

Blake, J., 1999. Overcoming the "value-action gap" in environmental policy: Tensions between national policy and local experience. Local Environment 4 (3): 257278.

Blaser, M., 2009. Political ontology: cultural studies without "cultures"? Cultural Studies 23 (5-6): 873896.

Blaser, M., 2010. Storytelling Globalization from the Chaco and Beyond. Durham and London: Duke University Press. 
Blaser, M., M. de la Cadena and A. Escobar, 2014. The Anthropocene and the One-world (or the Anthropos-not-seen). Unpublished manuscript .

Bloch, E., 1986. The Principle of Hope. 3 vols, trans. by $N$ and $S$. Plaice and P. Knight. Oxford, UK: Blackwell.

Bloch, E., 1988. The Utopian Function of Art and Literature: Selected Essays, ed. and trans. J. Zipes and F. Mecklenburg. Cambridge, Massachusetts: MIT Press.

Blomley, N., 2007. Critical geography: anger and hope. Progress in Human Geography 31 (1): 5365 .

Bochner, A. P., 2012. On first-person narrative scholarship: Autoethnography as acts of meaning. Narrative inquiry 22 (1): 155164.

Bohm, D., 1951. Quantum theory. Courier Corporation.

Bohm, D., 1996. Bohm on Dialogue. Routledge.

Bolt, B., 2010. Heidegger reframed: Interpreting key thinkers for the arts. IB Tauris.

Bonneuil, C. and J.-B. Fressoz, 2016. The shock of the Anthropocene: the Earth, history and us. Verso Books.

Bourdieu, P., 1993. The field of cultural production: Essays on art and literature. Columbia University Press.

Bourriaud, N., 1998. Relational Aesthetics. Les Presse Du Reel, France.

Bowen, G. A., 2009. Document analysis as a qualitative research method. Qualitative Research Journal 9 (2): 2740.

Boyatzis, R. E., 1998. Transforming Qualitative Information: Thematic analysis and code development. Sage.

Boyd, W., W. S. Prudham and R. A. Schurman, 2001. Industrial dynamics and the problem of nature. Society \& Natural Resources 14 (7): 555570.

Brace, C. and H. Geoghegan, 2011. Human geographies of climate change: Landscape, temporality, and lay knowledges. Progress in Human Geography 35 (3): 284302.

Brady, E., 1998. Imagination and the aesthetic appreciation of nature. The Journal of Aesthetics and Art Criticism 56 (2): 139147.

Brady, E., 2007. Aesthetic regard for nature in environmental and land art. Ethics, Place and Environment 10 (3): 287300.

Bratton, B., 2013. Some Trace Effects of the Post-Anthropocene: On Accelerationist Geopolitical Aesthetics. E-Flux Journal 46.

Braun, B., 2006. Towards a new earth and a new humanity: nature, ontology, politics. London: Blackwell. 
Braun, B., 2013. The 2013 Antipode RGS-IBG Lecture: New Materialisms and Neoliberal Natures. Antipode 47 (1): 114.

Braun, B., 2015a. Futures: Imagining Socioecological Transformation An Introduction. Annals of the Association of American Geographers 105 (2): 239243.

Braun, B., 2015b. Rethinking political ecology for the Anthropocene. In T. Perreault, G. Bridge and J. McCarthy, eds., The Routledge Handbook of Political Ecology, 102 114. Routledge.

Braun, B. P., 2014. A new urban dispositif? Governing life in an age of climate change. Environment and Planning D: Society and Space 32 (1): 4964.

Brigstocke, J., 2016. Exhausted Futures. GeoHumanities 2 (1): 92101.

British Council, 2010. Long Horizons: an exploration of art and climate change. British Council.

Broglio, R., 2013. When animals and technology are beyond human grasping. Angelaki: Journal of the theoretical humanities 18 (1): 19.

Bruce, S., 2002. God is Dead: Secularization in the West. Blackwell Oxford.

Brundtland, G., M. Khalid, S. Agnelli, S. Al-Athel, B. Chidzero, L. Fadika, V. Hauff, I. Lang, M. Shijun, M. M. de Botero et al., 1987. Our Common Future (Brundtland Report). United Nations.

Bryant, L. R., 2011. The Democracy of Objects. Open Humanities Press.

Buck, H. J., 2015. On the possibilities of a charming Anthropocene. Annals of the Association of American Geographers 105 (2): 369377.

Buell, L., 1996. The Environmental Imagination: Thoreau, Nature Writing, and the Formation of American culture. Cambridge, USA and London, UK: Harvard University Press.

Bussey, M., 2014. Intimate futures: bringing the body into futures work. European Journal of Futures Research 2 (1): 18.

Butler, J., 1990. Gender trouble: Feminism and the subversion of identity. Routledge.

Butler, J., 1997. Excitable speech: A politics of the performative. Psychology Press.

Cajete, G., 2000. Native science: Natural laws of interdependence. Clear Light Pub.

Cambray, J., 2002. Synchronicity and emergence. American Imago 59 (4): 409434.

Cameron, E., 2012. New geographies of story and storytelling. Progress in Human Geography 36 (5): 573592.

Cameron, E., S. de Leeuw and C. Desbiens, 2014. Indigeneity and ontology. Cultural Geographies 21 (1): 1926. 
Capra, F., 1996. The web of life: A new scientific understanding of living systems. Anchor.

Capstick, S., N. Pidgeon and M. Whitehead, 2013. Public perceptions of climate change in Wales: Summary findings of a survey of the Welsh public conducted during November and December 2012. Project Report.

Casarino, C., 2003. Time matters: Marx, Negri, Agamben, and the corporeal. Strategies 16 (2): 185206.

Castoriadis, C., 1994. Radical imagination and the social instituting imaginary. In G. Robinson and J. F. Rundell, eds., Rethinking Imagination: Culture and Creativity. London and New York: Routledge.

Castree, N., 2003. Commodifying what nature? Progress in Human Geography 27 (3): 273297.

Castree, N., 2005. Nature. London: Routledge.

Castree, N., 2013a. Making Sense of Nature. Routledge.

Castree, N., 2013b. Representing the Anthropocene: Who Will Get to Speak for Everything and How? URL https://www . youtube.com/watch?v=3Ks3nGYTVIY. Lastaccessed29July2017. Jon Murdoch Memorial Lecture, Cardiff University.

Castree, N., 2014a. The Anthropocene and Geography I: the back story. Geography Compass 8 (7): 436449 .

Castree, N., 2014b. The Anthropocene and Geography III: Future directions. Geography Compass 8 (7): 464476.

Castree, N., 2014c. Geography and the Anthropocene II: Current contributions. Geography Compass 8 (7): 450463.

Castree, N., 2015a. Changing the Anthropo (s) cene Geographers, global environmental change and the politics of knowledge. Dialogues in Human Geography 5 (3): 301316.

Castree, N., 2015b. Geographers and the Discourse of an Earth Transformed: Influencing the Intellectual Weather or Changing the Intellectual Climate? Geographical Research 53 (3): 244254.

Castree, N., 2015c. Geography and global change science: Relationships necessary, absent, and possible. Geographical Research 53 (1): 115.

Castree, N., 2017. Speaking for the "people disciplines": Global change science and its human dimensions. The Anthropocene Review 123.

Castree, N., P. A. Chatterton and N. Heynen, 2010. The Point is to Change it: Geographies of Hope and Survival in an Age of Crisis. John Wiley \& Sons. 
Castree, N. and G. Henderson, 2014. The capitalist mode of conservation, neoliberalism and the ecology of value. New Proposals: Journal of Marxism and Interdisciplinary Inquiry 7 (1): 1637.

Chakrabarty, D., 2009. The climate of history: four theses. Critical Inquiry 35 (2): 197222.

Chatterton, P., V. Gidwani, N. Heynen, A. Kent, W. Larner and R. Pain, 2011. Antipode in an antithetical era. Antipode 43 (2): 181189.

Cheney, J., 1999. The Journey Home. In A. Weston, ed., An Invitation to Environmental Philosophy, 141 168. Oxford, UK: Oxford University Press.

Chernilo, D., 2017. The question of the human in the Anthropocene debate. European Journal of Social Theory 20 (1): 4460.

Choy, T., 2011. Ecologies of comparison: An ethnography of endangerment in Hong Kong. Duke University Press.

Christophers, B., 2016. Risking value theory in the political economy of finance and nature. Progress in Human Geography 42 (3): 120.

Claisse, F. and P. Delvenne, 2015. Building on anticipation: Dystopia as empowerment. Current Sociology 63 (2): 155169.

Clark, N., 2017. Politics of strata. Theory, Culture \& Society 34 (2-3): 211231.

Clark, N. and Y. Gunaratnam, 2017. Earthing the Anthropos? From "socializing the Anthropocene" to geologizing the social. European Journal of Social Theory 20 (1): 146163.

Clark, P. U., J. D. Shakun, S. A. Marcott, A. C. Mix, M. Eby, S. Kulp, A. Levermann, G. A. Milne, P. L. Pfister, B. D. Santer et al., 2016. Consequences of twenty-firstcentury policy for multi-millennial climate and sea-level change. Nature climate change 6 (4): 360369.

Clarke, D. B., ed., 1997. The Cinematic City. Routledge.

Clarke, D. B., 2011. Utopologies. Environment and Planning D-Society 85 Space 29 (6): 951967.

Clifford, J., 2013. Returns: Becoming indigenous in the twenty-first century. Harvard University Press.

Clifford, N., S. French and G. Valentine, 2010. Key methods in geography. London: SAGE.

Cloke, P., I. Cook, P. Crang, M. Goodwin, J. Painter and C. Philo, 2004. Practising Human Geography. Sage.

Cloke, P. and O. Jones, 2001. Dwelling, place, and landscape: an orchard in Somerset. Environment and Planning A 33 (4): 649666. 
Closs Stephens, A., 2016. The affective atmospheres of nationalism. cultural geographies 23 (2): 181198.

Colebrook, C., 2014. We have always been post-Anthropocene. https://www . youtube.com/watch?v=o1jB7CI4y0k. Last accessed 8 December 2017. Presentation at the Center for 21 st Century Studies at the University of Wisconsin conference on Anthropocene Feminism, Milwaukee.

Coleman, E., 1989. The New Organic Grower. Chelsea Green .

Collard, R.-C., J. Dempsey and J. Sundberg, 2015. A Manifesto for Abundant Futures. Annals of the Association of American Geographers 105 (2): 322330.

Conley, V. A., 2008. Artists or "Little Soldiers?" Félix Guattari's Ecological Paradigms. In B. Herzogenrath, ed., Deleuze/Guattari and Ecology, 116128. AIAA.

Cooke, B., S. West and W. J. Boonstra, 2016. Dwelling in the biosphere: exploring an embodied human environment connection in resilience thinking. Sustainability Science 11 (5): 831843.

Coomaraswamy, A. K., 1935. The Transformation of Nature in Art. Harvard University Press.

Cooper, D. E., 2005. Heidegger on nature. Environmental Values 14 (3): 339351.

Cope, M., 2005. Coding Qualitative Data. In I. Hay, ed., Qualitative Research Methods in Human Geography, 223 33. London: Sage.

Costanza, R. and H. E. Daly, 1992. Natural capital and sustainable development. Conservation Biology 6 (1): 3746.

Costanza, R., R. d'Arge, R. De Groot, S. Farber, M. Grasso, B. Hannon, K. Limburg, S. Naeem, R. V. O'neill, J. Paruelo et al., 1997. The value of the world's ecosystem services and natural capital. Nature 387 (6630): 253260.

Costanza, R., L. Graumlich and W. L. Steffen, 2007. Sustainability or collapse? An integrated history and future of people on Earth. MIT Press.

Country, B., S. Wright, S. Suchet-Pearson, K. Lloyd, L. Burarrwanga, R. Ganambarr, M. Ganambarr-Stubbs, B. Ganambarr, D. Maymuru and J. Sweeney, 2016. Cobecoming Bawaka: Towards a relational understanding of place/space. Progress in Human Geography 40 (4): 455475.

Crang, M., 2003. Qualitative methods: touchy, feely, look-see? Progress in Human Geography 27 (4): 494504.

Cresswell, T., 2014. Geographies of poetry/poetries of geography. Cultural Geographies 21 (1): 141146. 
Crist, E., 2013. On the poverty of our nomenclature. Environmental Humanities 3: 129147.

Cronon, W., 1992. A place for stories: Nature, history, and narrative. The Journal of American History 78 (4): 13471376.

Cronon, W., 1996. The trouble with wilderness: or, getting back to the wrong nature. Environmental History 1 (1): 728.

Crouch, D., 2003. Performances and constitutions of natures: a consideration of the performance of lay geographies. In B. Szerszynski, W. Heim and C. Waterton, eds., Nature performed: Environment, Culture and Performance, 17 30. Blackwell.

Crouch, D., 2015. An Ecology of Values: critically interpreting John Newling's art. Cultural Geographies 22 (2): 361368.

Crutzen, P. J., 2002. Geology of mankind. Nature 415 (6867): 2323.

Crutzen, P. J. and E. Stoermer, 2000. The Anthropocene 41 (1): 1718.

Culler, J., 1982. The Pursuit of Signs: Semiotics, Literature, Deconstruction.

Culp, A., 2016. Dark Deleuze. University of Minnesota Press.

Cunsolo, A. and N. R. Ellis, 2018. Ecological grief as a mental health response to climate change-related loss. Nature Climate Change 8 (4): 275281.

Curtis, T., 1986. Wales the Imagined Nation: Essays in Cultural and National Identity. Bridgend: Poetry Wales Press.

Daily, G. and K. Ellison, 2002. The new economy of nature: the quest to make conservation profitable. Island Press.

Dalby, S., 2013. Biopolitics and climate security in the Anthropocene. Geoforum 49: 184192.

Dalby, S., 2015. Framing the Anthropocene: The good, the bad and the ugly. The Anthropocene Review 3351.

Davies, G. and C. Dwyer, 2007. Qualitative methods: are you enchanted or are you alienated? Progress in Human Geography 31 (2): 257266.

Davies, H., 2016. The Well-being of Future Generations (Wales) Act 2015: Duties or aspirations? Environmental Law Review 18 (1): 4156.

Davies, H., 2017. The Well-being of Future Generations (Wales) Act 2015: A Step Change in the Legal Protection of the Interests of Future Generations? Journal of Environmental Law 29 (1): 165175.

Davis, H. and E. Turpin, 2015. Art in the Anthropocene: Encounters Among Aesthetics, Politics, Environments and Epistemologies. London: Open Humanities Press. 
Dawney, L., 2011. Social imaginaries and therapeutic self-work: the ethics of the embodied imagination. The Sociological Review 59 (3): 535552.

de Botton, A., 2011. A Point of View (BBC Radio Four): The ecological sublime. http://www.bbc.co.uk/programmes/b00xj18g. Last accessed 21 May 2017.

De Castro, E. B. V., 2014. Cannibal Metaphysics: For a Post-Structural Anthropology, trans. by P. Skafish. Minneapolis, MN: Univocal.

De Certeau, M., 2011. The Practice of Everyday Life, 3rd edition. University of California Press.

De la Cadena, M., 2015. Earth beings: Ecologies of practice across Andean worlds. Duke University Press.

De Leeuw, S., 2012. Geographies of a Lover. NeWest Press.

De Leeuw, S., M. Greenwood and N. Lindsay, 2013. Troubling good intentions. Settler Colonial Studies 3 (3-4): 381394.

De Rosa, S. and J. Bluwstein, 2017. Why "Warning to Humanity" gets the socioecological crisis (and its solutions) wrong. https://entitleblog.org/2017/11/ 23/. Last accessed on 26.04.2018.

DeLanda, M., 2006. A new philosophy of society: Assemblage theory and social complexity. A\&C Black.

DeLanda, M., 2013. Intensive science and virtual philosophy. A\&C Black.

Delanty, G. and A. Mota, 2017. Governing the Anthropocene: Agency, governance, knowledge. European Journal of Social Theory 20 (1): 938.

Deleuze, G., 1994. Difference and Repetition. Columbia University Press.

Deleuze, G. and F. Guattari, 1986. Kafka. Toward a minor literature, trans. by D. Polan. University of Minnesota Press.

Deleuze, G. and F. Guattari, 1987. A Thousand Plateaus: Capitalism and Schizophrenia, trans. B. Massumi. University of Minnesota Press.

Deleuze, G. and F. Guattari, 2000. Anti-oedipus. Capitalism and Schizophrenia. University of Minnesota Press.

della Dora, V., 2015. The Mantle of the Earth: Surfaces, Landscape, Aesthetics. In H. Hawkins and E. Straughan, eds., Geographical Aesthetics: Imagining space, staging encounters, 225 249. Ashgate Publishing, Ltd.

Demeritt, D., 2009. Geography and the promise of integrative environmental research. Geoforum 2 (40): 127129.

Demos, T., 2013. Contemporary Art and the Politics of Ecology: An Introduction. Third Text 27 (1): 19. 
Department for Natural Resources of the Welsh Government, 2015. Well-being of Future Generations (Wales) Act 2015: The Essentials. https://gov . wales/docs/ dsjlg/publications/150623-guide-to-the-fg-act-en.pdf. Last accessed 8 June 2018.

Derrida, J., 1967. Of Grammatology. Baltimore: Johns Hopkins UP.

Derrida, J., 1977. Limited Inc. Northwestern University Press.

Derrida, J., 1991. "Eating Well" or the Calculation of the Subject: An Interview with Jacques Derrida. In E. Cadava, P. Conner and J.-L. Nancy, eds., Who Comes After the Subject?, 96 119. New York: Routledge.

Descola, P., 2013. Beyond nature and culture. University of Chicago Press.

Dewey, J., 1934. Art as Experience. Perigee Books.

Dewsbury, J., P. Harrison, M. Rose and J. Wylie, 2002. Enacting geographies. Geoforum $33(4)$ : 437440.

Dewsbury, J.-D., 2003. Witnessing space: knowledge without contemplation. Environment and Planning A 35 (11): 19071932.

Dewsbury, J. D., 2010. Performative, non-representational, and affect-based research. In A. S. Delyser D, Herbert S and C. MA, eds., The SAGE Handbook of Qualitative Geography, 321 344. Sage London.

Dittmer, J., 2014. Geopolitical assemblages and complexity. Progress in Human Geography 38 (3): 385401.

Dixon, D., H. Hawkins and E. Straughan, 2012. Of human birds and living rocks Remaking aesthetics for post-human worlds. Dialogues in Human Geography 2 (3): 249270.

Doel, M., 1994. Something Resists: Reading-Deconstruction as Ontological Infestation (Departures from the Texts of Jaques Derrida). In P. J. Cloke, M. A. Doel, D. Matless, N. Thrift and M. Phillips, eds., Writing the rural: five cultural geographies, 127 148. SAGE.

Doel, M., 2010. Analysing cultural texts. In N. Clifford, S. French and G. Valentine, eds., Key Methods in Geography, 485 496. London: SAGE.

Dryzek, J. S., 2013. The politics of the earth: Environmental discourses. Oxford University Press.

Edensor, T., 2000. Walking in the British countryside: reflexivity, embodied practices and ways to escape. Body \& Society 6 (3-4): 81106.

Edensor, T., D. Leslie, S. Millington and N. Rantisi, 2009. Spaces of vernacular creativity: Rethinking the cultural economy. Routledge. 
Ehlers, E. and T. Krafft, 2006. Earth System Science in the Anthropocene. Springer.

Ellis, E., 2011. The planet of no return: human resilience on an artificial earth. Breakthrough Journal 2: 3944.

Engelmann, S., 2015a. More-than-human affinitive listening. Dialogues in Human Geography 5 (1): 7679.

Engelmann, S., 2015b. Toward a poetics of air: sequencing and surfacing breath. Transactions of the Institute of British Geographers 40 (3): 430444.

England, K. V. L., 1994. Getting Personal: Reflexivity, Positionality, and Feminist Research. The Professional Geographer 46 (1): 241256.

Escobar, A., 1996. Construction nature: Elements for a post-structuralist political ecology. Futures 28 (4): 325343.

Escobar, A., 2011. Sustainability: Design for the pluriverse. Development 54 (2): 137140.

Eshun, G. and C. Madge, 2012. "Now let me share this with you": Exploring Poetry as a Method for Postcolonial Geography Research. Antipode 44 (4): 13951428.

Eshun, G. and C. Madge, 2016. Poetic world-writing in a pluriversal world: a provocation to the creative (re) turn in geography. Social \& Cultural Geography 18.

Esposito, R., [1998] 2010. Communitas: the origin and destiny of community, trans. by T. Campbell. Stanford University Press.

Evans, B. and J. Reid, 2014. Resilient life: the art of living dangerously. John Wiley \& Sons.

Falk, R., 2016. Climate change, policy knowledge, and the temporal imagination. In P. Wapner and H. Elver, eds., Reimagining Climate Change, 49 68. London and New York: Routledge.

Featherstone, M. and M. Miles, 2014. Utopias: An Introduction. Cultural Politics $10(2): 125131$.

Feindt, P. H. and A. Oels, 2005. Does discourse matter? Discourse analysis in environmental policy making. Journal of Environmental Policy \& Planning 7 (3): 161173.

Fischer-Kowalski, M. and H. Haberl, eds., 2007. Socioecological transitions and global change: Trajectories of social metabolism and land use. Edward Elgar Publishing.

Fisher, M., 2009. Capitalist Realism: Is there no alternative? Zero Books.

Fitznor, L., 2012. Indigenous Scholars and Writing Through Narratives and Storying for Healing and Bridging. In Hendry and L. Fitznor, eds., Anthropologists, Indigenous Scholars and the Research Endeavour., 270 283. Routledge. 
Flick, U., 2009. An Introduction to Qualitative Research. 4th edition. London: Sage.

Fløttum, K., A. M. Gjesdal, Ø. Gjerstad, N. Koteyko and A. Salway, 2014. Representations of the future in English language blogs on climate change. Global Environmental Change 29: 213222.

Flowers, M., L. Lipsett and M. Barrett, 2014. Animism, Creativity, and a Tree: Shifting into Nature Connection through Attention to Subtle Energies and Contemplative Art Practice. Canadian Journal of Environmental Education 19: 111126.

Folke, C., S. Carpenter, B. Walker, M. Scheffer, T. Chapin and J. Rockström, 2010. Resilience thinking: integrating resilience, adaptability and transformability. Ecology and society 15 (4).

Forde, E., 2017. The ethics of energy provisioning: Living off-grid in rural Wales. Energy Research \&3 Social Science 30: 8293.

Foster, J. B., 1999. Marx's theory of metabolic rift: classical foundations for environmental sociology. American Journal of Sociology 105 (2): 366405.

Foucault, M., [1966] 1970. The Order of Things. New York: Pantheon.

Foucault, M., 2002. The Archaeology of Knowledge. London and New York: Routledge Classics.

Fournier, V., 2002. Utopianism and the cultivation of possibilities: grassroots movements of hope. The Sociological Review 50 (S1): 189216.

Franklin, A., 2002. Nature and Social Theory. Sage.

Franklin, A., 2007. Posthumanism. In G. Ritzer, ed., Blackwell Encyclopedia of Sociology. Wiley Blackwell.

Fukuyama, F., 2006. The End of History and the Last Man. Simon and Schuster.

Gaard, G., ed., 1993. Ecofeminism: Women, animals, nature. Temple University Press.

Gaard, G., 2014. Mindful New Materialisms: Buddhist Roots for Material Ecocriticism's Flourishing. In S. Iovino and S. Oppermann, eds., Material Ecocriticism, 291 300. Indiana University Press.

Gaard, G. C., 2002. Vegetarian ecofeminism: A review essay. Frontiers: A Journal of Women Studies 23 (3): 117146.

Gablik, S., 1991. The Reenchantment of Art. Thames and Hudson New York.

Gabrys, J. and K. Yusoff, 2012. Arts, sciences and climate change: practices and politics at the threshold. Science as Culture 21 (1): 124. 
Gandy, M., 1997. Contradictory modernities: conceptions of nature in the art of Joseph Beuys and Gerhard Richter. Annals of the Association of American Geographers 87 (4): 636659.

Garforth, L., 2002. Green utopias: imagining the sustainable society. Unpublished PhD thesis, University of York.

Garforth, L., 2009. No intentions? Utopian theory after the future. Journal for Cultural Research 13 (1): 527.

Garforth, L. and P. Kraftl, 2009. Introduction: Utopia and the "Problem" of Intention. Journal for Cultural Research 13 (1): 14.

Gauntlett, D., 2013. Making is Connecting. John Wiley \& Sons.

Gaztambide-Fernández, R., 2014. Decolonial options and artistic/aestheSic entanglements: An interview with Walter Mignolo. Decolonization: Indigeneity, Education E Society 3 (1): 196212.

Gerlach, J., 2017a. Ecuador's experiment in living well: Sumak kawsay, Spinoza and the inadequacy of ideas. Environment and Planning A 49 (10): 22412260.

Gerlach, J., 2017b. Middle Hope. Cultural Geographies 24 (2): 333339.

Ghosh, A., 2016. The Great Derangement: Climate change and the unthinkable. University of Chicago Press.

Gibbs, G. R., 2007. Analysing Qualitative Data. Sage.

Gibson-Graham, J. and G. Roelvink, 2010. An economic ethics for the Anthropocene. Antipode 41 (s1): 320346.

Gibson-Graham, J. K., 2002. Beyond global vs. local: economic politics outside the binary frame. In A. Herod and M. Wright, eds., Geographies of power: Placing scale, 25 60. Blackwell Oxford.

Gibson-Graham, J.-K., 2008. Diverse economies: performative practices for "other worlds". Progress in Human Geography 32 (5): 613632.

Gibson-Graham, J. K., 2012. Diverse Economies: Performative Practices for "Other Worlds". In J. P. Trevor J. Barnes and E. Sheppard, eds., The Wiley-Blackwell Companion to Economic Geography, 33 46. Routledge London.

Gilbert, D. and D. Lambert, 2010. Counterfactual geographies: worlds that might have been. Journal of Historical Geography 36 (3): 245252.

Gillan, K. and J. Pickerill, 2012. The difficult and hopeful ethics of research on, and with, social movements. Social Movement Studies 11 (2): 133143.

Gillard, R., 2016. Questioning the Diffusion of Resilience Discourses in Pursuit of Transformational Change. Global Environmental Politics 16 (1): 1320. 
Gillard, R., A. Gouldson, J. Paavola and J. Van Alstine, 2016. Transformational responses to climate change: beyond a systems perspective of social change in mitigation and adaptation. Wiley Interdisciplinary Reviews: Climate Change 7 (2): 251265.

Ginn, F., 2015. When Horses Won't Eat: Apocalypse and the Anthropocene. Annals of the Association of American Geographers 105 (2): 351359.

Ginn, F., 2017. Posthumanism. In D. Richardson, N. Castree, M. Goodchild, A. Kobayashi, W. Liu and R. Marston, eds., The International Encyclopedia of Geography. Wiley Blackwell.

Glaser, B. G. and A. L. Strauss, 1967. The Discovery of Grounded Theory; Strategies for Qualitative Research. Chicago: IL: Aldine de Gruyter.

Glaser, M., G. Krause, B. Ratter and M. Welp, 2008. Human/Nature interaction in the anthropocene potential of social-ecological systems analysis. Gaia-Ecological Perspectives for Science and Society 17 (1): 7780.

Glăveanu, V. P., 2011. Creativity as cultural participation. Journal for the Theory of Social Behaviour 41 (1): 4867.

Goffman, E., 1959. The Presentation of Self in Everyday Life. Anchor Books.

Gold, J. R. and G. Revill, 2004. Representing the environment. Routledge.

Gray, J., 2007. Black mass: Apocalyptic religion and the death of utopia. Macmillan.

Greenhough, B. and E. Roe, 2010. From ethical principles to response-able practice. Environment and Planning D: Society and Space 28 (1): 4345.

Greenspan, H., 1998. On listening to Holocaust survivors: Recounting and life history. Praeger Publishers.

Gregory, D., 1994. Geographical Imaginations. Blackwell Oxford.

Gregory, D., R. Johnston, G. Pratt, M. Watts and S. Whatmore, 2009. The Dictionary of Human Geography. John Wiley \& Sons.

Griffiths, H. M., G. Goodwin, T. Keevil, E. Salisbury, S. Tooth and D. Roberts, 2017. Searching for an Anthropo (s) cene in the Uplands of Mid Wales. GeoHumanities .

Grimes, R. R., 2003. Ritual theory and the environment. In B. Szerszynski, W. Heim and C. Waterton, eds., Nature Performed: Environment, Culture, and Performance, 31 45. Blackwell.

Grosz, E., 1999. Becomings: Explorations in time, memory, and futures. Cornell University Press.

Grosz, E., 2008a. Darwin and feminism: Preliminary investigations for a possible alliance. Material feminisms 2351. 
Grosz, E. A., 2008b. Chaos, territory, art: Deleuze and the framing of the earth. Columbia University Press.

Groves, C., 2007. The Living Future in Philosophy. Working Paper. http://www . cardiff.ac.uk/socsi/futures/wp_cg_livingfuture121005.pdf. Last accessed 19 April 2017.

Groves, C., 2016. Emptying the future: On the environmental politics of anticipation. Futures .

Gruffudd, P., 1995. Remaking Wales: nation-building and the geographical imagination, 1925 1950. Political Geography 14 (3): 219239.

Guattari, F., [1989] 2014. The Three Ecologies. London and New York: Bloomsbury Publishing.

Guthman, J., 2014. Agrarian dreams: The paradox of organic farming in California. University of California Press.

Hajer, M. A., 1995. The politics of environmental discourse: ecological modernization and the policy process. Clarendon Press Oxford.

Halfacree, K., 2006. From dropping out to leading on? British counter-cultural backto-the-land in a changing rurality. Progress in Human Geography 30 (3): 309336.

Halfacree, K., 2007. Trial by space for a "radical rural": Introducing alternative localities, representations and lives. Journal of rural studies 23 (2): 125141.

Halfacree, K., 2011. "Alternative" communities in rural Wales. In P. Milbourne, ed., Rural Wales in the Twenty-First Century. Cardiff: University of Wales Press.

Hall, L., 2014. My Mother's Garden: Aesthetics, Indigenous Renewal, and Creativity. In H. Davis and E. Turpin, eds., Art in the Anthropocene: Encounters Among Aesthetics, Politics, Environments and Epistemologies, 283 292. London: Open Humanities Press.

Hallam, E. and T. Ingold, 2007. Creativity and Cultural Improvisation. Oxford: Berg.

Hammersley, M. and P. Atkinson, 1995. Ethnography: Principles in practice. Second ed. Routledge.

Hammersley, M. and P. Atkinson, 2007. Ethnography: Principles in practice. Third ed. Routledge.

Haraway, D., 1988. Situated knowledges: The science question in feminism and the privilege of partial perspective. Feminist Studies 14 (3): 575599.

Haraway, D., 1991. Simians, Cyborgs, and Women: The reinvention of nature. Free Association Books. 
Haraway, D., 1992. The promises of monsters: A regenerative politics for Inappropriate/d others. In L. Grossberg, C. Nelson and P. A. Treichler, eds., Cultural Studies, 295 337. New York: Routledge.

Haraway, D., 2015. Anthropocene, capitalocene, plantationocene, chthulucene: Making kin. Environmental Humanities 6 (1): 159165.

Haraway, D. J., 1994. A game of cat's cradle: Science studies, feminist theory, cultural studies. Configurations 2 (1): 5971.

Haraway, D. J., 2008. When Species Meet. University of Minnesota Press.

Haraway, D. J., 2016. Staying with the trouble: Making kin in the Chthulucene. Durham: Duke University Press.

Harney, L., J. McCurry, J. Scott and J. Wills, 2016. Developing "process pragmatism" to underpin engaged research in human geography. Progress in Human Geography 40 (3): 316333.

Harris, D. M., 2017a. Book review: The Great Derangement: Climate Change and the Unthinkable by Amitav Ghosh. Cultural Geographies 24 (4): 647654.

Harris, D. M., 2017b. Telling the story of climate change: Geologic imagination, praxis, and policy. Energy Research \& Social Science 31: 179183.

Harvey, D., 1990. Between Space and Time: Reflections on the Geographical Imagination1. Annals of the Association of American Geographers 80 (3): 418434.

Harvey, D., 1993. From space to place and back again: Reflections on the condition of postmodernity. In J. Bird, B. Curtis, T. Putnam and L. Tickner, eds., Mapping the futures: local cultures, global change, 2 29. Routledge.

Harvey, D., 1996. Justice, Nature and the Geography of Difference. Oxford: Blackwell.

Harvey, D., 2000. Spaces of Hope. Berkeley and Los Angeles: University of California Press.

Haven, E. and A. Khrennikov, 2013. Quantum social science. Cambridge University Press.

Hawkins, H., 2013. Geography and art. An expanding field Site, the body and practice. Progress in Human Geography 37 (1): 5271.

Hawkins, H., 2017. Creativity (Key Ideas in Geography). Routledge.

Hawkins, H., L. Cabeen, F. Callard, N. Castree, S. Daniels, D. DeLyser, H. M. Neely and P. Mitchell, 2015a. What might GeoHumanities do? Possibilities, practices, publics, and politics. GeoHumanities 1 (2): 211232.

Hawkins, H., S. A. Marston, M. Ingram and E. Straughan, 2015b. The Art of Socioecological Transformation. Annals of the Association of American Geographers 105 (2): 331341. 
Hawkins, H. and E. Straughan, 2015. Geographical Aesthetics: Imagining space, staging encounters. Ashgate Publishing.

Hayden, J. and D. Buck, 2012. Doing community supported agriculture: Tactile space, affect and effects of membership. Geoforum 43 (2): 332341.

Head, L., 2015. The Anthropoceneans. Geographical Research 53 (3): 313320.

Hebdige, D., 1993. Training some thougths on the future. In J. Bird, B. Curtis, T. Putnam and L. Tickner, eds., Mapping the futures: local cultures, global change, 270 279. Routledge.

Hector, D. C., C. B. Christensen and J. Petrie, 2014. Sustainability and sustainable development: Philosophical distinctions and practical implications. Environmental Values 23 (1): 728.

Heidegger, M., 1966. Memorial address (Discourse on thinking). Harper and Row.

Heidegger, M., 1971. Building Dwelling Thinking. In M. Heidegger, ed., Poetry, Language, Thought, trans. by A. Hofstadter. Harper and Row New York.

Heidegger, M., 1995. The Fundamental Concepts of Metaphysics. Trans. William McNeill and Nicholas Walker. Bloomington: Indiana University Press.

Hendry, P. M., 2007. The future of narrative. Qualitative Inquiry 13 (4): 487498.

Herman, R., 2008. Reflections on the importance of indigenous geography. American Indian Culture and Research Journal 32 (3): 7388.

Hicks, D., 1996. A lesson for the future: Young people's hopes and fears for tomorrow. Futures 28 (1): 113.

Hirt, P. W., 1996. A Conspiracy of Optimism: Management of the national forests since World War Two. USA: University of Nebraska Press.

Holden, M., 2010. The rhetoric of sustainability: perversity, futility, jeopardy? Sustainability 2 (2): 645659 .

Holling, C. S., 2001. Understanding the complexity of economic, ecological, and social systems. Ecosystems 4 (5): 390405.

Horton, J. L. and J. C. Berlo, 2013. Beyond the Mirror: Indigenous Ecologies and New Materialisms in Contemporary Art. Third Text 27 (1): 1728.

Howard Sir, A., 1947. The Soil and Health: A Study of Organic Agriculture. Schocken Books, New York.

Huehls, M., 2010. Structures of Feeling: Or, How to Do Things (or Not) with Books. Contemporary Literature 51 (2): 419428.

Hughes, J. D., 1985. Theophrastus as ecologist. Environmental History Review 9 (4): 296306. 
Hulme, M., 2011. Reducing the future to climate: a story of climate determinism and reductionism. Osiris 26 (1): 245266.

Hulme, M., S. Dessai, I. Lorenzoni and D. R. Nelson, 2009. Unstable climates: Exploring the statistical and social constructions of "normal" climate. Geoforum 40 (2): 197206.

Hurley, K., 2008. Is that a future we want? An ecofeminist exploration of images of the future in contemporary film. Futures 40 (4): 346359.

Ignatov, A., 2016. African Orature as Ecophilosophy: Tuning in to the Voices of the Land. GeoHumanities 2 (1): 7691.

Inayatullah, S., 1990. Deconstructing and reconstructing the future: predictive, cultural and critical epistemologies. Futures 22 (2): 115141.

Inayatullah, S., 1993. From "who am I?" to "when am I?": framing the shape and time of the future. Futures 25 (3): 235253.

Inayatullah, S., 1994. Life, the universe and emergence. Futures 26 (6): 683696.

Ingold, T., 1986. The appropriation of nature: essays on human ecology and social relations. Manchester University Press.

Ingold, T., 2000. The perception of the environment: essays on livelihood, dwelling and skill. Psychology Press.

Ingold, T., 2004. Culture on the ground the world perceived through the feet. Journal of Material Culture 9 (3): 315340.

Ingold, T., 2013. Dreaming of dragons: on the imagination of real life. Journal of the Royal Anthropological Institute 19 (4): 734752.

Ingold, T., 2014. That's enough about ethnography! HAU: Journal of Ethnographic Theory 4 (1): 383395.

Ingold, T. and J. L. Vergunst, 2008. Ways of walking: Ethnography and practice on foot. Ashgate Publishing, Ltd.

Ingram, M., 2007. Biology and beyond: The science of "back to nature" farming in the United States. Annals of the Association of American Geographers 97 (2): 298312 .

Ingram, M., 2014. Washing urban water: diplomacy in environmental art in the Bronx, New York City. Gender, Place \& Culture 21 (1): 105122.

Ingram, M., H. Ingram and R. Lejano, 2014. What's the story? Creating and sustaining environmental networks. Environmental Politics 23 (6): 9841002.

Jackson, M., 2013. The other shore: Essays on writers and writing. University of California Press. 
Jackson, M., 2014. Composing postcolonial geographies: Postconstructivism, ecology and overcoming ontologies of critique. Singapore Journal of Tropical Geography 35 (1): 7287.

Jackson, M., 2016. Aesthetics, Politics, and Attunement: On Some Questions Brought by Alterity and Ontology. GeoHumanities 2 (1): 823.

Jackson, M. and M. Fannin, 2011. Letting Geography Fall Where it May Aerographies Address the Elemental. Environment and Planning D: Society and Space 29 (3): 435444.

Jameson, F., 1996. The Seeds of Time. Columbia University Press.

Jameson, F., 2005. Archeologies of the Future: The Desire Called Utopia and Other Science. Verso.

Jansson, A. M., 1994. Investing in natural capital: the ecological economics approach to sustainability. Island Press.

Jasanoff, S. and S.-H. Kim, 2009. Containing the atom: Sociotechnical imaginaries and nuclear power in the United States and South Korea. Minerva 47 (2): 119.

Jasanoff, S. and S.-H. Kim, 2013. Sociotechnical imaginaries and national energy policies. Science as Culture 22 (2): 189196.

Johnson, E., H. Morehouse, S. Dalby, J. Lehman, S. Nelson, R. Rowan, S. Wakefield and K. Yusoff, 2014. After the Anthropocene Politics and geographic inquiry for a new epoch. Progress in Human Geography 118.

Jones, O., 2009. After nature: Entangled worlds. In N. Castree, D. Demeritt, D. Liverman and B. Rhoads, eds., A Companion to Environmental Geography, 294312. Wiley-Blackwell.

Jones, O. and P. Cloke, 2008. Non-human agencies: trees in place and time. In C. Knappett and L. Malafouris, eds., Material Agency: Towards a NonAnthropocentric Approach, 79 96. Springer.

Joseph Rowntree Foundation, 2015. Monitoring Poverty and Social Exclusion in Wales 2015. https://www.jrf.org.uk/report/monitoring-poverty-andsocial-exclusion-wales-2015. Last accessed 8 June 2018.

Joseph Rowntree Foundation, 2016. Prosperity without poverty: A framework for action in Wales. https://www.jrf . org.uk/report/prosperity-without-poverty. Last accessed 8 June 2018.

Jung, C. G., 1952. Synchronicity: An Acausal Connecting Principle. Rascher verlag, Zurich.

Jung, C. G. and C. A. Pauli, Wolfgang. Ed. Meier, 2001. Atom and Archetype: the Pauli/Jung Letters, 1932-1958. Princeton University Press. 
Kagan, S., 2014. Art and sustainability: Connecting patterns for a culture of complexity. transcript Verlag.

Kallis, G., 2011. In defence of degrowth. Ecological Economics 70 (5): 873880.

Kallis, G. and H. March, 2015. Imaginaries of hope: The utopianism of degrowth. Annals of the Association of American Geographers 105 (2): 360368.

Kanngieser, A., 2012. A sonic geography of voice towards an affective politics. Progress in Human Geography 36 (3): 336353.

Katz, C., 1995. Major/minor: Theory, nature, and politics. Annals of the Association of American Geographers 85 (1): 164168.

Katz, C., 1996. Towards minor theory. Environment and Planning D: Society and Space 14 (4): 487499.

Katz, C., 2001. Vagabond capitalism and the necessity of social reproduction. Antipode 33 (4): 709728.

Keats, J., 1899. The complete poetical works and letters of John Keats. Houghton, Mifflin and company.

Kenny, M., 2007. Introduction: Exploring "the utopian" in political ideologies. Journal of Political Ideologies 12 (3): 211217.

Kenny, M., 2014. Anthropocene, Capitalocene, Chthulhucene: Donna Haraway in conversation with Martha Kenney. In H. Davis and E. Turpin, eds., Art in the Anthropocene: Encounters Among Aesthetics, Politics, Environments and Epistemologies, 255 270. London: Open Humanities Press.

Kershaw, B., 2012. Performance ecologies, biotic rights and retro-modernisation. Research in Drama Education: The Journal of Applied Theatre and Performance 17 (2): 265287.

Kester, G. H., 2004. Conversation pieces: Community and communication in modern art. Univ of California Press.

King, T., 2003. The truth about stories: A native narrative. House of Anansi.

Kinsley, S., 2010. Representing "things to come": Feeling the visions of future technologies. Environment and Planning A 42 (11): 27712790.

Klein, N., 2007. The Shock Doctrine: The rise of disaster capitalism. Macmillan.

Klein, N., 2015. This Changes Everything: Capitalism vs. The Climate. New York: Simon and Schuster.

Klinke, I., 2013. Chronopolitics: A conceptual matrix. Progress in Human Geography 37 (5): 673690. 
Kohn, E., 2013. How forests think: Toward an anthropology beyond the human. Univ of California Press.

Kolinjivadi, V., G. Van Hecken, D. V. Almeida, J. Dupras and N. Kosoy, 2017. Neoliberal performatives and the "making" of Payments for Ecosystem Services (PES). Progress in Human Geography 123.

Kollmuss, A. and J. Agyeman, 2002. Mind the gap: why do people act environmentally and what are the barriers to pro-environmental behavior? Environmental education research 8 (3): 239260.

Korten, D. C., 1995. When Corporations Rules the World. Kumarian Press (World wide).

Kraftl, P., 2007. Utopia, performativity, and the unhomely. Environment and Planning D: Society and Space 25 (1): 120143.

Kroeber, K., 1997. Ecology and American Literature: Thoreau and Un-Thoreau. American Literary History 9 (2): 309328.

Kropotkin, P., [1902] 2012. Mutual Aid: A Factor of Evolution. Courier Corporation.

Kunnas, J., 2017. Storytelling: From the early Anthropocene to the good or the bad Anthropocene. The Anthropocene Review 4 (2): 136150.

Lack, A., 2014. Martin Heidegger on Technology, Ecology, and the Arts. Springer.

Larsen, S. C. and J. T. Johnson, 2016. The Agency of Place: Toward a More-ThanHuman Geographical Self. GeoHumanities 118.

Last, A., 2015. Introduction to "Decolonising the Anthropocene". Presentation at University of Westminster, 27 November 2015.

Last, A., 2017. We are the world? Anthropocene cultural production between geopoetics and geopolitics. Theory, Culture \& Society 34 (2-3): 147168.

Latimer, J. and B. Skeggs, 2011. The politics of imagination: keeping open and critical. The Sociological Review 59 (3): 393410.

Latour, B., 1993. We Have Never Been Modern, trans. by C. Porter. Harvard University Press.

Latour, B., 2004a. How to talk about the body? The normative dimension of science studies. Body \&5 society 10 (2-3): 205229.

Latour, B., 2004b. Why has critique run out of steam? From matters of fact to matters of concern. Critical Inquiry 30 (2): 225248.

Latour, B., 2008. It's development, Stupid! or: How to modernize modernization. In J. Proctor, ed., Postenvironmentalism. Cambridge MA: MIT Press. 
Latour, B., 2011. From multiculturalism to multinaturalism: What rules of method for the new socio-scientific experiments? Nature and Culture 6 (1): 117.

Latour, B., 2014. Agency at the Time of the Anthropocene. New Literary History $45(1): 118$.

Latour, B., 2015. Fifty shades of green. Environmental Humanities 7 (1): 219225.

Laurier, E., 2016. Participant Observation. In N. Clifford, M. Cope, S. French and T. Gillespie, eds., Research Methods in Human and Physical Geography. 3rd edition., 169 181. London: SAGE.

Law, J., 2004. After method: Mess in social science research. Routledge.

Lawson, V., 2007. Geographies of care and responsibility. Annals of the Association of American Geographers 97 (1): 111.

Le Guin, U. K., 2004. The Wave in the Mind: Talks and Essays on the Writer, the Reader, and the Imagination. Shambhala Publications.

Legat, A., 2008. Walking Stories; Leaving Footprints. In T. Ingold and J. L. Vergunst, eds., Ways of walking: Ethnography and practice on foot, 35 49. Ashgate Publishing, Ltd.

Lejano, R., M. Ingram and H. Ingram, 2013. The Power of Narrative in Environmental Networks. Cambridge MA: MIT Press.

Leopold, A., 1949. A Sand County Almanac. Oxford University Press New York.

Leuthold, S., 1998. Indigenous aesthetics: Native art, media, and identity. University of Texas Press.

LeVasseur, T., 2016. Introduction: Religion, Agriculture, and Sustainability. University Press of Kentucky.

Levene, M., 2010. The Apocalyptic as Contempory Dialectic: from Thanatos (violence) to Eros (transformation). In S. Skrimshire, ed., Future ethics: Climate change and apocalyptic imagination, 59 81. Bloomsbury Publishing.

Lévi-Strauss, C., 1963. Structural anthropology. Basic Books.

Levitas, R., 1993. The future of thinking about the future. In J. Bird, B. Curtis, T. Putnam and L. Tickner, eds., Mapping the Futures: local cultures, global change, 257 266. London and New York: Routledge.

Levitas, R., 2013. Utopia as Method: The Imaginary Reconstitution of Society. Basingstoke, UK: Palgrave macmillan.

Lewis, S. L. and M. A. Maslin, 2015. Defining the anthropocene. Nature 519 (7542): 171180. 
Loftus, A., 2009. Intervening in the environment of the everyday. Geoforum 40 (3): 326334.

Lorenzoni, I. and M. Hulme, 2009. Believing is seeing: laypeople's views of future socio-economic and climate change in England and in Italy. Public Understanding of Science 18 (4): 383400.

Lorimer, H., 2003. Telling small stories: spaces of knowledge and the practice of geography. Transactions of the Institute of British Geographers 28 (2): 197217.

Lorimer, H., 2005. Cultural geography: the busyness of being "more-thanrepresentational". Progress in Human Geography 29 (1): 8394.

Lorimer, H., 2006. Herding memories of humans and animals. Environment and planning D: society and space 24 (4): 497518.

Lorimer, H., 2008. Cultural geography: non-representational conditions and concerns. Progress in Human Geography .

Lorimer, H., 2009. Caught in the nick of time: Archives and fieldwork. In M. Crang, D. Delyser, S. Herbert and L. McDowell, eds., The SAGE Handbook of Qualitative Geography, 248 273. Sage London.

Lorimer, H., 2011. Walking: new forms and spaces for studies of pedestrianism. In T. Cresswell and P. Merriman, eds., Geographies of mobilities: Practices, spaces, subjects, chapter 2, 19 33. Ashgate Publishing.

Lorimer, H. and H. Parr, 2014. Excursions telling stories and journeys. Cultural Geographies 21 (4): 543547.

Lorimer, J., 2012. Multinatural geographies for the Anthropocene. Progress in Human Geography 36 (5): 593612.

Lorimer, J., 2015. Wildlife in the Anthropocene: conservation after nature. University of Minnesota Press.

Lorimer, J., T. Hodgetts and M. Barua, 2017. Animals' atmospheres. Progress in Human Geography 120.

Lovelock, J., 1995. The Ages of Gaia: A biography of our living earth. Oxford University Press, USA.

Mack, N., C. Woodsong, K. M. MacQueen, G. Guest and E. Namey, 2005. Qualitative research methods: a data collectors field guide. Durham NC: Family Health Interventions.

MacKinnon, D. and K. D. Derickson, 2013. From resilience to resourcefulness: A critique of resilience policy and activism. Progress in Human Geography 37 (2): 253270 . 
Macy, J. and C. Johnstone, 2012. Active Hope: How to face the mess we're in without going crazy. California: New World Library.

Madge, C., 2014. On the creative (re) turn to geography: poetry, politics and passion. Area 46 (2): 178185.

Magrane, E., 2015. Situating geopoetics. GeoHumanities 1 (1): 86102.

Magrane, E., 2017. Creative Geographies and Environments: Geopoetics in the Anthropocene. (PhD Thesis). https://arizona.openrepository.com/handle/ 10150/624580. Last accessed 2 May 2018.

Malm, A. and A. Hornborg, 2014. The geology of mankind? A critique of the Anthropocene narrative. The Anthropocene Review 6269.

Mansfield, B., C. Biermann, K. McSweeney, J. Law, C. Gallemore, L. Horner and D. K. Munroe, 2015. Environmental politics after nature: conflicting socioecological futures. Annals of the Association of American Geographers 105 (2): 284293.

Mansfield, B. and M. Doyle, 2017. Nature: A Conversation in Three Parts. Annals of the American Association of Geographers 107 (1): 2227.

Marcus, G. E., 1995. Technoscientific imaginaries: Conversations, profiles, and memoirs. University of Chicago Press.

Marcuse, H., 1966. Eros and Civilization: Philosopical Inquiry Into Freud. Beacon Press.

Marcuse, H., 1972. Ecology and Revolution. Liberation 16: 1012.

Marcuse, H., 1992. Ecology and the critique of modern society. Capitalism Nature Socialism 3 (3): 2938.

Marcuse, H., 2007. Art and Liberation: collected papers of Herbert Marcuse. Routledge.

Marshall, G., 2014. Hearth and Hiraeth: Constructing Climate Change Narratives around National Identity.

Marston, S. A. and S. De Leeuw, 2013. Creativity and geography: Toward a politicized intervention. Geographical Review 103 (2).

Marston, S. A., J. P. Jones and K. Woodward, 2005. Human geography without scale. Transactions of the Institute of British Geographers 30 (4): 416432.

Marx, K., 1976. Capital: A Critique of Political Economy. Vol. 1. 4th edn Penguin Books.

Massey, D., 1991. A Global Sense of Place. Marxism Today 38: 2429.

Massey, D., 1992. Politics and space/time. New Left Review (196): 65. 
Massey, D., 1993. Power-geometry and a progressive sense of place. In J. Bird, B. Curtis, T. Putnam and L. Tickner, eds., Mapping the futures: local cultures, global change, 60 70. Routledge.

Massey, D., 1994. Space, Place and Gender. Cambridge, UK and Malden, USA: Polity Press.

Massey, D., 1999. Space time, "science" and the relationship between physical geography and human geography. Transactions of the Institute of British Geographers 24 (3): 261276.

Massey, D., 2004. Geographies of responsibility. Geografiska Annaler: Series B, Human Geography 86 (1): 518.

Massey, D., 2005. For Space. London, Thousand Oaks, New Delhi: SAGE Publications.

Massey, D., 2013a. Social Science Bites podcast: Doreen Massey on Space. http: //www. socialsciencespace.com/2013/02/podcastdoreen-massey-on-space/. Last accessed 19 April 2017.

Massey, D., 2013b. Vocabularies of the economy. Soundings 54 (54): 922.

Massey, D. and N. Thrift, 2003. The passion of place. In R. Johnston and M. Williams, eds., A Century of British Geography, 275 299. Oxford University Press Oxford.

Mathews, F., 2003. For love of matter: A contemporary panpsychism. SUNY Press.

Mathews, F., 2006. Beyond modernity and tradition: a third way for development. Ethics 8 the Environment 11 (2): 85113.

Maxey, L., 2003. One path forward? Three sustainable communities in England and Wales. Unpublished PhD thesis, Department of Geography, University of Wales, Swansea.

McAfee, K., 2016. Green economy and carbon markets for conservation and development: a critical view. International Environmental Agreements: Politics, Law and Economics 16 (3): 333353.

McCormack, D. P., 2008. Engineering affective atmospheres on the moving geographies of the 1897 Andrée expedition. Cultural Geographies 15 (4): 413430.

McKibben, B., 1989. The End of Nature. Random House Incorporated.

McKibben, B., 2005. Can you imagine? A warming world needs art. https://www.opendemocracy.net/globalization-climate_change_debate/ article_2447.jsp. Last accessed 8 June 2018.

Mclntosh, A., 2012. The Challenge of Radical Human Ecology to the Academy. In L. Williams, R. A. Roberts and A. McIntosh, eds., Radical human ecology: Intercultural and indigenous approaches, 31 56. Ashgate Publishing. 
McNally, D., 2015. Comforting Others: Sociality and the Ethical Aesthetics of BeingTogether. In H. Hawkins and E. Straughan, eds., Geographical Aesthetics: Imagining space, staging encounters, 121 136. Ashgate Publishing, Ltd.

Meadows, D. H., D. Meadows, J. Randers and W. W. Behrens, 1972. The Limits to Growth: A Report for the Club of Rome's Project on the Predicament of Mankind. Potomac Associates - Universe Books.

Mentz, S., 2012. After sustainability. PMLA 127 (3): 586592.

Merchant, C., 1980. The Death of Nature: Women, Ecology, and Scientific Revolution. Harper, San Francisco.

Mignolo, W. and R. Vásquez, 2013. Decolonial aestheSis: Colonial wounds/decolonial healings. http://socialtextjournal.org/periscope topic/decolonial_aesthesis. Last accessed 8 March 2017.

Miles, M., 2010. Representing nature: art and climate change. Cultural Geographies 17 (1): 1935 .

Miles, M., 2014. Eco-aesthetics: art, literature and architecture in a period of climate change. Bloomsbury Publishing.

Miles, M., 2016. Eco-aesthetic dimensions: Herbert Marcuse, ecology and art. Cogent Arts $\mathscr{G}$ Humanities 3 (1): 117.

Milkoreit, M., 2016. The Promise of Climate Fiction: Imagination, Storytelling and the Politics of the Future. In P. Wapner and H. Elver, eds., Reimagining Climate Change, 171 191. London and New York: Routledge.

Millennium Ecosystem Assessment, 2005. Ecosystems and Human Well-Being: Synthesis. Island Press, Washington, DC.

Miller, C. A., J. O'Leary, E. Graffy, E. B. Stechel and G. Dirks, 2015. Narrative futures and the governance of energy transitions. Futures 70: 6574.

Minkowski, E., 1970. Lived time: Phenomenological and psychopathological studies. Northwestern University Press.

Moezzi, M., K. B. Janda and S. Rotmann, 2017. Using stories, narratives, and storytelling in energy and climate change research. Energy Research $\mathscr{E}$ Social Science 31: 110 .

Moore, J. W., 2015. Capitalism in the Web of Life: Ecology and the Accumulation of Capital. Verso Books.

Moore, J. W., 2017. The Capitalocene, Part I: On the nature and origins of our ecological crisis. The Journal of Peasant Studies 44 (3): 594630. 
Moore, M.-L., O. Tjornbo, E. Enfors, C. Knapp, J. Hodbod, J. Baggio, A. Norström, P. Olsson and D. Biggs, 2014. Studying the complexity of change: toward an analytical framework for understanding deliberate social-ecological transformations. Ecology and Society 19 (4).

Moore, S. A., J. Wilson, S. Kelly-Richards and S. A. Marston, 2015. School gardens as sites for forging progressive socioecological futures. Annals of the Association of American Geographers 105 (2): 407415.

Morton, T., 2007. Ecology without nature: Rethinking environmental aesthetics. Harvard University Press.

Morton, T., 2010. The Ecological Thought. Cambridge, USA and London, UK: Harvard University Press.

Morton, T., 2013. Hyperobjects: Philosophy and Ecology after the End of the World. University of Minnesota Press.

Moser, S. C., 2006. Talk of the city: engaging urbanites on climate change. Environmental research letters 1 (1): 014006.

Moser, S. C. and L. Dilling, 2011. Communicating climate change: closing the scienceaction gap. In J. Dryzek, R. Norgaard and D. Schlosberg, eds., The Oxford handbook of climate change and society, 161 174. Oxford University Press.

Mott, C. and D. Cockayne, 2017. Citation matters: mobilizing the politics of citation toward a practice of 'conscientious engagement'. Gender, Place \&6 Culture 24 (7): 120 .

Murdoch, J., 1997. Inhuman/nonhuman/human: actor-network theory and the prospects for a nondualistic and symmetrical perspective on nature and society. Environment and planning D: Society and Space 15 (6): 731756.

Murphy, A. B., 2013. Advancing geographical understanding Why engaging grand regional narratives matters. Dialogues in Human Geography 3 (2): 131149.

Naess, A., 1989. Ecology, Community, and Lifestyle: Outline of an Ecosophy, translated and revised by D. Rothenberg. Cambridge: Cambridge University Press.

Nash, C., 2000. Performativity in practice: some recent work in cultural geography. Progress in Human Geography 24 (4): 653664.

National Assembly for Wales Research Service, 2012. Quick Guide: Ecosystems and Ecosystem Approach. http://www.assembly.wales/Research\%20Documents/ Ecosystems $\% 20$ and $\% 20$ the $\% 20$ Ecosystem $\% 20$ Approach $\% 20-\% 20$ Quick $\% 20$ guide08032012-231338/qg12-0006-English.pdf Last accessed 8 December 2017.

Natural Resources Wales, 2013. Our Purpose and Priorities 2013 2014. Welsh Government. 
Natural Resources Wales, 2017. Introducing Sustainable Management of Natural Resources. https://naturalresources .wales/media/678317/introducing-smnrbooklet-english.pdf. Last accessed 8 December 2017.

Neal, L., 2015. Playing for Time: making art as if the world mattered. Oberon Books.

Neocleous, M., 2012. "Don't Be Scared, Be Prepared": Trauma-Anxiety-Resilience. Alternatives 37 (3): 188198.

Netherwood, A., 2008. Futures, Trends and Horizon Scanning for Welsh Local Government. Welsh Local Government Association.

Nietzsche, F. W., 2000. The birth of tragedy. Oxford University Press, USA.

Nordblad, J., 2017. Time for politics: How a conceptual history of forests can help us politicize the long term. European Journal of Social Theory 20 (1).

Nordhaus, T. and M. Shellenberger, 2007. Break Through: From the Death of Environmentalism to the Politics of Possibility. New York: Houghton Mifflin Company.

Nordmann, A., 2014. Responsible innovation, the art and craft of anticipation. Journal of Responsible Innovation 1 (1): 8798.

Norgaard, R. B., 2010. Ecosystem services: From eye-opening metaphor to complexity blinder. Ecological economics 69 (6): 12191227.

Noxolo, P., 2017. Decolonial theory in a time of the re-colonisation of UK research. Transactions of the Institute of British Geographers 42 (3): 342344.

Núñez, R. E. and E. Sweetser, 2006. With the future behind them: Convergent evidence from Aymara language and gesture in the crosslinguistic comparison of spatial construals of time. Cognitive science 30 (3): 401450.

O'Brien, K., 2011. Responding to environmental change: A new age for human geography? Progress in Human Geography 35 (4): 542549.

O'Brien, K. L., 2016. Climate change and social transformations: is it time for a quantum leap? Wiley Interdisciplinary Reviews: Climate Change 7 (5): 618626.

Ogborn, M., A. Blunt, P. Gruffudd, D. Pinder and J. May, eds., 2003. Cultural geography in practice. Edward Arnold, London.

O'Hare, P. and I. White, 2013. Deconstructing Resilience: Lessons from Planning Practice. Planning Practice \&5 Research 28 (3): 275279.

Osborne, T., 2003. Against "creativity": a philistine rant. Economy and society 32 (4): 507525.

Ostrom, E., 2009. A general framework for analyzing sustainability of social-ecological systems. Science 325 (5939): 419422. 
Pachauri, R. K., M. R. Allen, V. R. Barros, J. Broome, W. Cramer, R. Christ, J. A. Church, L. Clarke, Q. Dahe, P. Dasgupta et al., 2014. Climate change 2014: synthesis report. Contribution of Working Groups I, II and III to the fifth assessment report of the Intergovernmental Panel on Climate Change. IPCC.

Padrutt, H., 2009. Heidegger and Ecology. In L. McWhorter and G. Stenstad, eds., Heidegger and the Earth: essays in environmental philosophy, 17 44. University of Toronto Press.

Parr, H., 1998. Mental health, ethnography and the body. Area 30 (1): 2837.

Paull, J., 2011. Biodynamic agriculture: The journey from Koberwitz to the world, 1924-1938. Journal of Organic Systems 6 (1): 2741.

Peeples, J., 2011. Toxic sublime: Imaging contaminated landscapes. Environmental Communication: A Journal of Nature and Culture 5 (4): 373392.

Peterson, A., 2016. Religion, Local Community, and Sustainable Agriculture. In V. Shiva, Y. Deutscher, L. Hurtado, E. Pembamoyo, M. Lemons, J. Adhikari, P. Jain, A. W. Sanford, M. H. Dixon, R. Sutterfield et al., eds., Religion and Sustainable Agriculture: World Spiritual Traditions and Food Ethics, 233 249. University Press of Kentucky.

Phelan, J. and P. J. Rabinowitz, 2008. A companion to narrative theory. John Wiley \& Sons.

Phillips, P., 2015. Artistic practices and ecoaesthetics in post-sustainable worlds. In C. Crouch, N. Kaye and J. Crouch, eds., An introduction to sustainability and aesthetics: The arts and design for the environment, 55 68. Boca Raton, Florida: Brown Walker Press.

Pickerill, J., 2015. Building the commons in eco-communities. In S. Kirwan, L. Dawney and J. Brigstocke, eds., Space, Power and the Commons: The Struggle for Alternative Futures. Routledge.

Pickerill, J. and L. Maxey, 2007. The Lammas low impact housing development. Sustainability: The practical journal for green building, renewable energy and sustainable communities 1: 1819.

Pickerill, J. and L. Maxey, 2009. Geographies of sustainability: low impact developments and radical spaces of innovation. Geography Compass 3 (4): 15151539.

Pile, S., 2010. Emotions and affect in recent human geography. Transactions of the Institute of British Geographers 35 (1): 520.

Pinder, D., 2011. Errant paths: the poetics and politics of walking. Environment and Planning D: Society and Space 29 (4): 672692.

Plumwood, V., 1993. Feminism and the mastery of nature. United Kingdom: Routledge. 
Plumwood, V., 2009. Nature in the Active Voice. Australian Humanities Review 46: 113129.

Polak, F. L. and E. Boulding, 1973. The Image of the Future. Elsevier.

Polletta, F., 2006. It was like a fever. Storytelling in Protest and Politics .

Pollock, V. L. and J. P. Sharp, 2007. Constellations of identity: place-ma (r) king beyond heritage. Environment and Planning D: Society and Space 25 (6): 1061 1078 .

Popper, K., 1962. The Open Society and its Enemies. Harper and Row.

Prigogine, I. and I. Stengers, 1984. Order Out of Chaos: Man's new dialogue with nature. Bantam books New York.

Prigogine, I. and I. Stengers, 1997. The End of Certainty: Time, Chaos and the New Laws of Nature. New York: The Free Press.

Pugh, J., 2014. Resilience, complexity and post-liberalism. Area 46 (3): 313319.

Purdy, J., 2015. After Nature: a politics for the Anthropocene. Harvard University Press.

Radcliffe, S. A., 2017a. Decolonising geographical knowledges. Transactions of the Institute of British Geographers 42 (3): 329333.

Radcliffe, S. A., 2017b. Geography and indigeneity I: Indigeneity, coloniality and knowledge. Progress in Human Geography 41 (2): 220229.

Rajchman, J., 1999. Diagram and Diagnosis. In E. Grosz, ed., Becomings: Explorations in time, memory, and futures, 42 54. Cornell University Press.

Ramsden, H., 2017. Walking \& talking: making strange encounters within the familiar. Social \& Cultural Geography 18 (1): 5377.

Randalls, S., 2015. Creating positive friction in the Anthropo(s)cenes. Dialogues in Human Geography 5 (3): 333336.

Rappaport, R. A., 1999. Ritual and Religion in the Making of Humanity. Cambridge University Press.

Reason, P., 2007. Education for ecology: Science, aesthetics, spirit and ceremony. Management Learning 38 (1): 2744.

Rees, W. E., 1998. How should a parasite value its host? Ecological Economics 25: 4952.

Reganold, J. P., A. S. Palmer, J. C. Lockhart and A. N. Macgregor, 1993. Soil quality and financial performance of biodynamic and conventional farms in New Zealand. Science 260 (5106): 344344. 
Rickards, L., R. Ison, H. Fünfgeld and J. Wiseman, 2014. Opening and closing the future: climate change, adaptation, and scenario planning. Environment and Planning C: Government and Policy 32 (4): 587602.

Riessman, C. K., 1993. Narrative Analysis. Sage.

Rigby, K., 2014. Spirits That Matter: Pathways towards a rematerialization of Religion and Spirituality. In S. Iovino and S. Oppermann, eds., Material Ecocriticism, 283 290. Indiana University Press.

Ripple, W. J., C. Wolf, T. M. Newsome, M. Galetti, M. Alamgir, E. Crist, M. I. Mahmoud, W. F. Laurance and . s. s. f. . c. 15, 2017. World Scientists' Warning to Humanity: A Second Notice. BioScience 67 (12): 10261028.

Ritchie, J., J. Lewis, C. M. Nicholls, R. Ormston et al., 2013. Qualitative research practice: A guide for social science students and researchers. Sage.

Robertson, M., 2012. Measurement and alienation: making a world of ecosystem services. Transactions of the Institute of British Geographers 37 (3): 386401.

Robertson, M. M., 2000. No net loss: wetland restoration and the incomplete capitalization of nature. Antipode 32 (4): 463493.

Robertson, M. M., 2006. The nature that capital can see: science, state, and market in the commodification of ecosystem services. Environment and Planning D: society and space 24 (3): 367387.

Robinson, J., 2004. Squaring the circle? Some thoughts on the idea of sustainable development. Ecological economics 48 (4): 369384.

Rockström, J., J. D. Sachs, M. C. Öhman and G. Schmidt-Traub, 2013. Sustainable Development and Planetary Boundaries. http://www.post2015hlp. org/wp-content/uploads/2013/06/Rockstroem-Sachs-Oehman-SchmidtTraub_Sustainable-Development-and-Planetary-Boundaries.pdf.Last accessed 8 December 2017.

Rockström, J., W. Steffen, K. Noone, Å. Persson, F. S. Chapin, E. F. Lambin, T. M. Lenton, M. Scheffer, C. Folke, H. J. Schellnhuber et al., 2009. A safe operating space for humanity. Nature 461 (7263): 472475.

Roe, E., 1994. Narrative policy analysis: Theory and practice. Duke University Press.

Rogers, A., 2011. Butterfly takes flight: the translocal circulation of creative practice. Social \& Cultural Geography 12 (7): 663683.

Romanyshyn, R. D., 1989. Technology as a Symptom \& Dream. New York: Routledge.

Rorty, R., 1982. Consequences of pragmatism: Essays, 1972-1980. University of Minnesota Press.

Rorty, R., 2009. Philosophy and the Mirror of Nature. Princeton University Press. 
Rose, M., 2006. Gathering "dreams of presence": a project for the cultural landscape. Environment and Planning D: Society and Space 24 (4): 537554.

Rose, M., 2010. Pilgrims: an ethnography of sacredness. Cultural Geographies 17 (4): 507524 .

Rose, M., 2014. Negative governance: vulnerability, biopolitics and the origins of government. Transactions of the Institute of British Geographers 39 (2): 209223.

Rose, M., 2016. A Place for Other Stories: Authorship and Evidence in Experimental Times. GeoHumanities 117.

Rossiter, P., 2002. Imagining Responsibility: Who are "we" anyway? Continuum: Journal of Media \& Cultural Studies 16 (1): 8194.

Ruby, J., 1991. Speaking for, speaking about, speaking with, or speaking alongside An anthropological and documentary dilemma. Visual Anthropology Review 7 (2): 5067.

Saldanha, A., 2012. Aestheticism and post-humanism. Dialogues in Human Geography 2 (3): 276279.

Saldanha, A., 2013. Some principles of geocommunism. Unpublished manuscript, http://www.geocritique.org/arun-saldanha-some-principles-of-geocommunism/ (last accessed 12 November 2017).

Sanjek, R., 1990. Fieldnotes: The makings of anthropology. Cornell University Press.

Sargisson, L., 1996. Contemporary feminist utopianism. Psychology Press.

Schafer, R. M., 1991. The Theatre of Confluence II. Descant 22 (2): 87103.

Schechner, R., 1988. Performance theory. Routledge.

Schieffelin, E. L., 1998. Problematizing performance. In F. Hughes-Freeland, ed., Ritual, performance, media, 194 207. Routledge.

Schmidt, R. W., H. Lamm and G. Trommsdorff, 1978. Social class and sex as determinants of future orientation (time perspective) in adults. European Journal of Social Psychology 8 (1): 7190.

Scranton, R., 2015. Learning to Die in the Anthropocene: Reflections on the End of a Civilization. City Lights Publishers.

Seale, C., 1999. The Quality of Qualitative Research. Oxford: Blackwell.

Serres, M., 1982. The Parasite, trans. by LR. Schehr. Baltimore: John Hopkins University.

Serres, M., 1995. Genesis, trans. by G. James and J. Nielson. University of Michigan Press. 
Sharp, J., 2007. The life and death of five spaces: public art and community regeneration in Glasgow. Cultural Geographies 14 (2): 274292.

Shaw, C. and B. Nerlich, 2015. Metaphor as a mechanism of global climate change governance: A study of international policies, 1992 2012. Ecological Economics 109: 3440 .

Sheridan, J. and R. Longboat, 2006. The Haudenosaunee imagination and the ecology of the sacred. Space and Culture 9 (4): 365381.

Shirani, F., K. Parkhill, C. Butler, C. Groves, N. Pidgeon and K. Henwood, 2016. Asking about the future: methodological insights from energy biographies. International Journal of Social Research Methodology 19 (4): 429444.

Shor, I. and P. Freire, 1987. A pedagogy for liberation: Dialogues on transforming education. Greenwood Publishing Group.

Silverman, D., 2010. Doing Qualitative Research: A Practical Handbook. 3rd Edition. London: SAGE.

Silverman, D., 2013. Doing Qualitative Research: A Practical Handbook. 4th Edition. SAGE Publications Limited.

Simms, A. and J. Smith, 2008. Do Good Lives Have to Cost the Earth? Constable.

Simon, S. and S. Randalls, 2016. Geography, ontological politics and the resilient future. Dialogues in Human Geography 6 (1): 318.

Simpson, L., 2015. Islands of decolonial love: Stories 83 songs. Arp Books.

Sjögren, H., 2016. Sustainability for Whom? The Politics of Imagining Environmental Change in Education. (PhD Thesis). Linköping University. Available at https://www.diva-portal.org/smash/get/diva2:923244/FULLTEXT01.pdf. Accessed 2 May 2018.

Skocz, D., 2009. Environmental Management in the "Age of the World Picture". In L. McWhorter and G. Stenstad, eds., Heidegger and the Earth: essays in environmental philosophy, 123 44. University of Toronto Press.

Skrimshire, S., 2010. Future ethics: Climate change and apocalyptic imagination. Bloomsbury Publishing.

Smith, N., 1984. Uneven development: Nature, capital, and the production of space. Oxford: Blackwell.

Smith, R. G., 2011. Materialism, Ecology, Aesthetics. Mediations 25 (2): 6178.

Smith, T. A., 2017. Witchcraft, spiritual worldviews and environmental management: Rationality and assemblage. Environment and Planning A 49 (3): 592611.

Solnit, R., 2003. Acts of Hope. https://orionmagazine.org/article/acts-ofhope/. Last accessed 8 June 2018. 
Solnit, R., 2005. Hope in the Dark: The untold history of people power. Canongate Books.

Sontag, S., 2002. Styles of Radical Will. Picador USA; Reprint.

Soper, K., 2008. Alternative hedonism, cultural theory and the role of aesthetic revisioning. Cultural Studies 22 (5): 567587.

Sparke, M., 2007. Geopolitical fears, geoeconomic hopes, and the responsibilities of geography. Annals of the Association of American Geographers 97 (2): 338349.

Springer, S., 2017. Earth writing. GeoHumanities 3 (1): 119.

Stanley, L. and S. Wise, 1993. Breaking Out Again: Feminist Ontology and Epistemology. Routledge.

Steffen, W., K. Richardson, J. Rockström, S. E. Cornell, I. Fetzer, E. M. Bennett, R. Biggs, S. R. Carpenter, W. de Vries, C. A. de Wit et al., 2015. Planetary boundaries: Guiding human development on a changing planet. Science 347 (6223): 110.

Steffen, W., R. A. Sanderson, P. D. Tyson, J. Jäger, P. A. Matson, B. Moore III, F. Oldfield, K. Richardson, H. J. Schellnhuber, B. L. Turner et al., 2006. Global Change and the Earth System: A Planet Under Pressure. Springer Science \& Business Media.

Steiner, R., [1924]1993. Spiritual foundations for the renewal of agriculture. Kimberton, PA: Bio-Dynamic Farming and Gardening Association.

Stengers, I., 2005. The Cosmopolitical Proposal. In B. Latour and P. Weibel, eds., Making things public: Atmospheres of democracy, 994 1003. MIT Press Cambridge.

Stenstad, G., 2009. Down-to-Earth Mystery. In L. McWhorter and G. Stenstad, eds., Heidegger and the Earth: essays in environmental philosophy, 236 252. University of Toronto Press.

Stirling, A., 2016. Nexus methods: presentation to the Royal Geographical Society Annual Conference 2016. https://www. slideshare.net/Stepscentre/andystirling-nexus-methods-rgs-2016. Last accessed on 3 July 2017.

Stoetzler, M. and N. Yuval-Davis, 2002. Standpoint theory, situated knowledge and the situated imagination. Feminist theory 3 (3): 315333.

Strauss, K., 2015. These overheating worlds. Annals of the Association of American Geographers 105 (2): 342350.

Strydom, P., 2017. The sociocultural self-creation of a natural category: Socialtheoretical reflections on human agency under the temporal conditions of the Anthropocene. European Journal of Social Theory 20 (1): 6179. 
Suarez, D., 2013. You cannot manage what you do not measure: Natural capital accounting at Rio +20 . Presentation to the Association of American Geographers, Los Angeles, California.

Sullivan, S., 2009. Green capitalism, and the cultural poverty of constructing nature as service-provider. Radical Anthropology 3: 1827.

Sullivan, S., 2010. "Ecosystem Service Commodities" A New Imperial Ecology? Implications for Animist Immanent Ecologies, with Deleuze and Guattari. New Formations 69 (1): 111128.

Sundberg, J., 2014. Decolonizing posthumanist geographies. Cultural Geographies 21 (1): 3347.

Swyngedouw, E., 2013a. Apocalypse Now! Fear and Doomsday Pleasures. Capitalism Nature Socialism 24 (1): 918.

Swyngedouw, E., 2013b. The non-political politics of climate change. ACME 12 (1): 18.

Szerszynski, B., 2017. Gods of the Anthropocene: geo-spiritual formations in the Earth's new epoch. Theory, Culture, and Society 34 (2 3): 253275.

Szerszynski, B., W. Heim and C. Waterton, 2003. Nature performed: Environment, Culture and Performance. Blackwell.

Taylor, C., 2004. Modern social imaginaries. Duke University Press.

Taylor, P. J., 1982. A materialist framework for political geography. Transactions of the Institute of British Geographers 7: 1534.

Thacker, E., 2015. Cosmic Pessimism. Minneapolis: Univocal Publishing.

Thompson, J., 2009. Performance Affects. Palgrave Macmillan.

Thrift, N., 1999. Steps to an Ecology of Place. In D. B. Massey, J. Allen and P. Sarre, eds., Human Geography Today, 295 322. Polity Press Cambridge.

Thrift, N., 2004. Intensities of feeling: towards a spatial politics of affect. Geografiska Annaler: Series B, Human Geography 86 (1): 5778.

Thrift, N., 2008. Non-representational theory: Space, politics, affect. Routledge.

Thrift, N., 2011. Lifeworld Inc and what to do about it. Environment and Planning D: Society and Space 29 (1): 526.

Todd, Z., 2014. Indigenizing the Anthropocene. In H. Davis and E. Turpin, eds., Art in the Anthropocene: Encounters Among Aesthetics, Politics, Environments and Epistemologies, 241 254. London: Open Humanities Press.

Todd, Z., 2016. An indigenous feminist's take on the ontological turn: "ontology" is just another word for colonialism. Journal of Historical Sociology 29 (1): 422. 
Tomlinson, J., 2007. The Culture of Speed: The Coming of Immediacy. London: Sage.

Tonn, B., A. Hemrick and F. Conrad, 2006. Cognitive representations of the future: survey results. Futures 38 (7): 810829.

Tuck, E. and K. W. Yang, 2012. Decolonization is not a metaphor. Decolonization: Indigeneity, Education \& Society 1 (1): 140.

Turner, V., 1969. Liminality and Communitas. Aldine Chicago.

Ukeles, M. L., 1969. Manifesto for Maintenance Art. https://www .arnolfini .org. $\mathrm{uk} / \mathrm{blog} / \mathrm{manifesto-for-maintenance-art-1969.} \mathrm{Last} \mathrm{accessed} 8$ June 2018.

Unger, R. M., 2004. False necessity: Anti-necessitarian social theory in the service of radical democracy, Revised Edition. Verso, London.

United Nations Report of the Secretary-General, 2013. Intergenerational solidarity and the needs of future generations. https://sustainabledevelopment.un.org/ content/documents/2006future.pdf. Last accessed 8 June 2018.

Uprichard, E., 2011. Narratives of the future: complexity, time and temporality. The Sage Handbook of Innovation in Social Research Methods 103: 119.

Urry, J., 2003. Global Complexity. Polity Cambridge.

Urry, J., 2005a. The Complexities of the Global. Theory, Culture 85 Society 22 (5): 235254.

Urry, J., 2005b. The Complexity Turn. Theory, Culture \&f Society 22 (5): 114.

Urry, J., 2011. Climate Change and Society. Polity.

Vannini, P., 2012. Ferry tales: Mobility, place, and time on Canada's west coast. Routledge.

Vaughan, L., 2009. Walking the line: Affectively understanding and communicating the complexity of place. The Cartographic Journal 46 (4): 316322.

Veland, S. and A. H. Lynch, 2016. Scaling the Anthropocene: How the stories we tell matter. Geoforum 72: 15.

Venkatesh, S., 2009. Gang leader for a day. Penguin UK.

Vira, B., 2015. Taking natural limits seriously: Implications for development studies and the environment. Development and Change 46 (4): 762776.

Virilio, P., 2006. Speed and Politics. Semiotexte.

Volk, T., 2003. Gaia's Body: Toward a Physiology of Earth. MIT Press.

Von Bertalanffy, L., 1956. General Systems Theory. Yearbook of the society for the Advancement of General System Theory 1: 110. 
Wainwright, J. and G. Mann, 2013. Climate Leviathan. Antipode 45 (1): 122.

Wainwright, J. and G. Mann, 2015. Climate change and the adaptation of the political. Annals of the Association of American Geographers 105 (2): 313321.

Walker, J. and M. Cooper, 2011. Genealogies of resilience: From systems ecology to the political economy of crisis adaptation. Security Dialogue 42 (2): 143160.

Wallen, R., 2012. Ecological art: a call for visionary intervention in a time of crisis. Leonardo 45 (3): 234242.

Wallin, J. J., 2017. Pedagogy at the brink of the post-anthropocene. Educational Philosophy and Theory 49 (11): 10991111.

Wampole, C., 2016. Rootedness: The Ramifications of a Metaphor. University of Chicago Press.

Wapner, P., 2016. Introduction: reimagining climate change. In P. Wapner and H. Elver, eds., Reimagining Climate Change, 1 13. London and New York: Routledge.

Waters, C. N., J. Zalasiewicz, C. Summerhayes, A. D. Barnosky, C. Poirier, A. Gałuszka, A. Cearreta, M. Edgeworth, E. C. Ellis, M. Ellis et al., 2016. The Anthropocene is functionally and stratigraphically distinct from the Holocene. Science 351 (6269): aad2622.

Watts, V., 2013. Indigenous place-thought and agency amongst humans and non humans (First Woman and Sky Woman go on a European world tour!). Decolonization: Indigeneity, Education $\&$ Society 2 (1).

Weber, M., [1948] 2009. From Max Weber: essays in sociology. Routledge.

Weintraub, L., A. C. Danto and T. McEvilley, 1996. Art on the edge and over: searching for art's meaning in contemporary society, 1970s-1990s. Art Insights.

Weisman, A., 2008. The World Without Us. Macmillan.

Welsh Assembly Government, 2009. One Wales: One Planet. The Sustainable Development Scheme of the Welsh Assembly Government. https://gov.wales/docs/ desh/publications/090521susdev1wales1planeten.pdf. Last accessed 8 June 2018.

Welsh Assembly Government, 2010. Climate Change Strategy for Wales. https: //gov.wales/docs/desh/publications/101006ccstratfinalen.pdf. Last accessed 8 June 2018.

Wendt, A., 2015. Quantum Mind and Social Science: Unifying Physical and Social Ontology. Cambridge University Press.

Whatmore, S., 1999. Rethinking the "human" in human geography. In D. Massey, J. Allen and P. Sarre, eds., Human Geography Today, 22 41. Polity Press, Cambridge. 
Whatmore, S., 2002. Hybrid Geographies: Natures, Cultures, Spaces. Sage.

Whatmore, S., 2005. Generating materials. In M. Pryke, G. Rose and S. Whatmore, eds., Using Social Theory, 89 104. Sage Publications with the Open University.

Whatmore, S., 2006. Materialist returns: practising cultural geography in and for a more-than-human world. Cultural Geographies 13 (4): 600609.

Whatmore, S. and S. Hinchliffe, 2003. Living cities: making space for urban nature. Soundings 3750.

White, I. and P. O'Hare, 2014. From Rhetoric to Reality: Which Resilience, Why Resilience, and Whose Resilience in Spatial Planning? Environment and Planning C: Government and Policy 32 (5): 934950.

White, K., 1992. Elements of geopoetics. Edinburgh Review 88: 163178.

White Jr, L., 1967. 4. The Historical Roots of Our Ecologic Crisis. Science 155 (3767): 12031207.

Whitehead, A. N., 2010. Process and reality. Simon and Schuster.

Whiteley, A., A. Chiang and E. Einsiedel, 2016. Climate Change Imaginaries? Examining Expectation Narratives in Cli-Fi Novels. Bulletin of Science, Technology E6 Society 36 (1): 2837.

Whyman, T., 2014. What is cupcake fascism? http://www.full-stop. net/2014/04/03/blog/tom-whyman/what-is-cupcake-fascism/. Last accessed 8 June 2018.

Williams, N., 2016. Creative processes: From interventions in art to intervallic experiments through Bergson. Environment and Planning A 48 (8): 15491564.

Williams, R., 1976. Keywords: A vocabulary of culture and society. Fontana.

Williams, R., 1977. Marxism and Literature. Oxford Paperbacks.

Williams, R., 1989. Resources of Hope. Verso.

Woodyer, T. and H. Geoghegan, 2013. (Re) enchanting geography? The nature of being critical and the character of critique in human geography. Progress in Human Geography 37 (2): 195214.

Worth, S. and J. Adair, 1972. Through Navajo Eyes an Exploration in Film Communication and Anthropology. Bloomington: Indiana UP.

Wylie, J., 2005. A single day's walking: narrating self and landscape on the South West Coast Path. Transactions of the Institute of British geographers 30 (2): 234 247.

Yusoff, K., 2009. Excess, catastrophe, and climate change. Environment and planning. D, Society and space 27 (6): 1010. 
Yusoff, K., 2013. Geologic life: prehistory, climate, futures in the Anthropocene. Environment and Planning D: Society and Space 31 (5): 779795.

Yusoff, K., 2017. Geosocial strata. Theory, Culture \& Society 34 (2-3): 105127.

Yusoff, K. and J. Gabrys, 2011. Climate change and the imagination. Wiley Interdisciplinary Reviews: Climate Change 2 (4): 516534.

Zalasiewicz, J., C. Waters and M. J. Head, 2017. Anthropocene: its stratigraphic basis. Nature 541 (7637): 289289.

Zimmerer, K. S., 1994. Human geography and the "new ecology": The prospect and promise of integration. Annals of the Association of American Geographers 84 (1): 108125.

Zimmerman, J. M. and V. Coyle, 1996. The Way of Council. Bramble Books.

Žižek, S., 2011. Living in the end times. Verso. 